Refining a model-based assessment strategy to estimate the ammonia emission from floors in dairy cow houses 


\section{Propositions}

1. Manure scraping does not contribute to lowering ammonia emission from dairy cow houses.

(this thesis)

2. It is more effective to estimate ammonia emission from a dairy cow house with a model than to measure this emission.

(this thesis)

3. Results of laboratory research only, do not contribute to solutions that improve the sustainability of food production.

4. The health effects of being in nature overpower the negative effects of air pollution.

5. To finish a $\mathrm{PhD}$ thesis succesfully, feeling well is the most important input variable.

6. The best way to find out your current mood is to look at your dog.

Propositions belonging to the thesis entitled:

'Refining a model-based assessment strategy to estimate the ammonia emission from floors in dairy cow houses'

Dennis Snoek

Wageningen, 27 October 2016 
Refining a model-based assessment strategy

to estimate the ammonia emission

from floors in dairy cow houses

Dennis J.W. Snoek 


\section{Thesis committee}

\section{Promotor}

Prof. Dr P.W.G. Groot Koerkamp

Professor of Farm Technology

Wageningen University

\section{Co-promotors}

Dr N.W.M. Ogink

Senior Scientist, Wageningen UR Livestock Research

Dr J.D. Stigter

Assistant Professor, Biometris

Wageningen University

\section{Other members}

Prof. Dr O. Oenema, Wageningen University

Dr G. Zhang, Aarhus University, Denmark

Dr P. Demeyer, Institute for Agricultural and Fisheries Research (ILVO), Merelbeke, Belgium

Dr G.J. Monteny, Monteny Milieu Advies, Renkum

This research was conducted under the auspices of the Graduate School WIAS (Wageningen Institute of Animal Sciences) 


\title{
Refining a model-based assessment strategy to estimate the ammonia emission from floors in dairy cow houses
}

\author{
Dennis J.W. Snoek
}

\section{Thesis}

submitted in fulfilment of the requirements for the degree of doctor at Wageningen University by the authority of the Rector Magnificus

Prof. Dr A.P.J. Mol,

in the presence of the

Thesis Committee appointed by the Academic Board

to be defended in public

on Thursday 27 October 2016

at 11 a.m. in the Aula. 
Dennis J.W. Snoek

Refining a model-based assessment strategy to estimate the ammonia emission from floors in dairy cow houses

184 pages.

PhD thesis, Wageningen University, Wageningen, NL (2016)

With references, with summary in English

ISBN: 978-94-6257-885-2

DOI: http://dx.doi.org/10.18174/387486 


\section{Table of Contents}

Page

1 General Introduction . . . . . . . . . . . . . . . . . . 7

2 Sensitivity analysis . . . . . . . . . . . . . . . . . 19

3 Puddle $\mathrm{pH}$ method and measurements . . . . . . . . . . . . . . . 45

4 Puddle depth method . . . . . . . . . . . . . . . . . 63

5 Puddle area method . . . . . . . . . . . . . . . . . 75

6 Assessing puddle area and depth . . . . . . . . . . . . . . . 89

7 Assessing puddle $\mathrm{pH}$ and urea concentration . . . . . . . . . . . . . . 111

8 General Discussion . . . . . . . . . . . . . . . . . . . 137

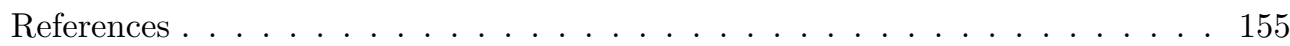

Summary . . . . . . . . . . . . . . . . . . . . 169

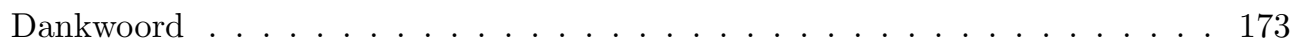

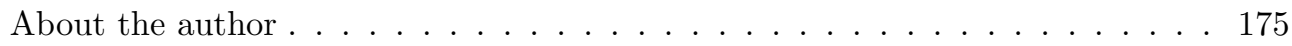

List of publications . . . . . . . . . . . . . . . . . 177

Training and Supervision Plan . . . . . . . . . . . . . . . 179 



\section{CHAPTER 1}

\section{General Introduction}

\section{Dennis Snoek}

In the eighties of the previous century it became clear that the deposition of ammonia $\left(\mathrm{NH}_{3}\right)$ is an important contributor to the acidification, eutrophication and nitrification of the environment (van Breemen et al., 1982; van Breemen \& van Dijk, 1988; Buijsman et al., 1987). The acidification impact can be significant with damage to forests, crops and other vegetation, and negative effects at aquatic ecosystems. The eutrophication can lead to reduced water quality, and in this way to decreased biodiversity, changes in species, and toxicity effects. Finally, $\mathrm{NH}_{3}$ is also an indirect source of the greenhouse gas nitrous oxide $\left(\mathrm{N}_{2} \mathrm{O}\right)$ (IPCC, 1996). So, there are some serious challenges caused by $\mathrm{NH}_{3}$.

The $\mathrm{NH}_{3}$ emission in western Europe in 1990 was estimated to be between 2.8 and $5.2 \mathrm{Mt} \mathrm{yr}^{-1}$ with the main contribution by livestock, specifically from livestock houses and manure storages (ECETOC, 1994). It was a quite rough estimate with an uncertainty range of at least $30 \%$, caused by a lack of data. It was also indicated that $\mathrm{NH}_{3}$ emission increased for about $50 \%$ from 1950 to 1980 and peaked in 1990 . In the late nineties, the Gothenburg Protocol of the UN Convention on Long-range Transboundary Air Pollution (UNECE, 1999) and the EU National Emission Ceiling Directive (NECD) (EU, 2001), among others, set $\mathrm{NH}_{3}$ emission ceilings EU wide and for each member country, for the year 2010. The other pollutants addressed in the NECD were nitrogen oxides $\left(\mathrm{NO}_{\mathrm{x}}\right)$, non-methane volatile organic compounds (NMVOCs) and sulphur dioxide $\left(\mathrm{SO}_{2}\right)$. From 1990 to 2010 the $\mathrm{NH}_{3}$ emission was reduced EU wide with $28 \%$ and the set targets were achieved by each member country (EEA, 2012). The European Commission (EC) proposed a new European Clean Air Programme with an updated NECD for the year 2020 and beyond (EEA, 2015). It 
was also reported that six member states, including The Netherlands, exceeded their ceilings in 2010 to 2013 , which was primarily due to recalculations in the agriculture sector (EEA, 2015). To summarise, the focus still has to be on the reduction of $\mathrm{NH}_{3}$ emission.

In addition, it was reported (EEA, 2015) that agriculture still dominates the $\mathrm{NH}_{3}$ emission, being about $95 \%$ for the total emissions in the EU-27. Compared to the other pollutants addressed in the NECD, the $\mathrm{NH}_{3}$ emissions did not decrease to the same extent since 1990 (EEA, 2015). In The Netherlands, cattle was the main agricultural category of $\mathrm{NH}_{3}$ emission in 2009, contributing for about $50 \%$ to total agriculture, followed by pigs $(25 \%)$ and poultry (15\%) (Velthof et al., 2012).

Concerning cattle emissions, major contributors are the housing and manure application. Cattle housing contributed for about $20 \%$ to total agriculture $\mathrm{NH}_{3}$ emissions (Velthof et al., 2012). In the Netherlands, cattle mainly consist of dairy cows. Therefore, this research focuses on the $\mathrm{NH}_{3}$ emission from dairy cow houses.

A typical dairy cow house in The Netherlands consists of, among others, a housing facility for the cows and a storage facility for the slurry. The storage facility, or so-called slurry pit, is generally underneath the complete cow house. A concrete slatted floor is used as walking and living area for the cows and there are cubicles for them to rest.

Since the eighties a lot of research has been performed related to the $\mathrm{NH}_{3}$ emission from livestock, and dairy cow houses in particular. This research is summarised in the following five sections:

- Section $1.1-\mathrm{NH}_{3}$ emission measurement methods

- Section $1.2-\mathrm{NH}_{3}$ emission process

- Section 1.3 - $\mathrm{NH}_{3}$ emission modelling

- Section $1.4-\mathrm{NH}_{3}$ emission mitigation methods

- Section 1.5 - $\mathrm{NH}_{3}$ emission reduction approach - status quo in The Netherlands

Having read these five sections, it becomes clear that we already know a lot about $\mathrm{NH}_{3}$, but that there are still challenges that need to be addressed. In Section 1.6 the objectives and the outline of this thesis are summarised.

\section{$1.1 \mathrm{NH}_{3}$ emission measurement methods}

With measurements it is possible to estimate the $\mathrm{NH}_{3}$ emission of a complete dairy cow house. To do this, both the total air exchange rate and the difference in $\mathrm{NH}_{3}$ concentrations of incoming and exhaust air have to be determined. Then the $\mathrm{NH}_{3}$ emission can be calculated according to Eq. (1.1).

$$
E_{N H_{3}}=Q \cdot \Delta x_{N H_{3}} \cdot t_{t o t}
$$


with $E_{N H_{3}}$ the $\mathrm{NH}_{3}$ emission [kg]; $Q$ the ventilation rate $\left[\mathrm{m}^{3} \mathrm{~h}^{-1}\right] ; \Delta x_{N_{3}}$ the $\mathrm{NH}_{3}$ concentration difference between in- and exhaust air $\left[\mathrm{kg} \mathrm{m}^{-3}\right]$; and $t_{\text {tot }}$ the total measurement time $[\mathrm{h}]$;

To measure the $\mathrm{NH}_{3}$ concentration, various methods were developed (van Ouwerkerk, 1993; Phillips et al., 2000, 2001; Mosquera et al., 2005b). However, only a few methods are currently applied in The Netherlands, as indicated by an asterisk (*) (Ogink et al., 2013): $\mathrm{NH}_{3}$-converter in combination with a $\mathrm{NH}_{3}$-analyser ${ }^{*}$; non-dispersive infra-red photometry; hyphenated laser photo acoustics ${ }^{*}$; filter pack system; denuder systems ${ }^{*}$; gas wash bottle*; gas detection tube; electrochemical cell; differential optic absorption spectroscopy (DOAS); fourier transform infra red (FTIR); open-pad tune-able diode laser (TDL)(Mosquera et al., 2005a)* ; passive measurement methods* .

Depending on the type of cow house, specific methods were applied. For example, in naturally ventilated buildings an anemometer is not applicable to assess the ventilation rate, in this case a tracer method should be used. However, the prerequisites to apply a tracer method, which needs sufficient extent of difference in gas concentration between inside and outside, may not be met in very open buildings, and its application may lead to unreliable measurements (Ogink et al., 2013).

To measure the air exchange rate, various methods can be applied: (1) gas or mass balances, with artificial tracers like $\mathrm{SF}_{6}$ or local produced tracers like $\mathrm{CO}_{2}$ or water vapour; (2) heat or energy balance; or (3) flow measurements at outlet or inlet openings with free impelled turbines or hot wire anemometers (Phillips et al., 2000, 2001). It is also possible to use modelling approaches, e.g. Computational Fluid Dynamics (CFD) (see Section 1.3)(Mosquera et al., 2005b; van Buggenhout et al., 2009). The general mass balance to determine the air exchange rate is described by Eq. (1.2) (van Buggenhout et al., 2009):

$$
V \cdot \frac{d C}{d t}+Q \cdot C(t)=m
$$

with $V$ the total volume of the ventilated space $\left[\mathrm{m}^{3}\right] ; d C / d t$ the concentration change of the tracer over time $t\left[\mathrm{~kg} \mathrm{~m}^{-3}\right] ; m$ the injection rate or the production rate of the tracer $\left[\mathrm{kg} \mathrm{h}^{-1}\right]$; and $t$ the time $[\mathrm{h}]$. The ventilation rate $Q$ can be calculated in case $V$, $C$ and $m$ are known.

Another method to determine the $\mathrm{NH}_{3}$ emission of a dairy cow house is to use a flux chamber to measure the emission from one or more selected areas in the house. A flux chamber is based on conservation of mass to determine the measured gasses from specific measurement areas. Flux chambers can vary in size, they usually cover a floor area of approximately $0.25 \mathrm{~m}^{2}$ to $1.00 \mathrm{~m}^{2}$, but a principal difference is the application as static or dynamic (Mosquera et al., 2005b). With static measurements a chamber is installed, air is circulated within the closed system, and concentration measurements are performed with certain time intervals. In a dynamic measurement air is soaked 
or blown over the emitting area in the flux chamber. Both methods are cheap and simple, but they both yield only emission rates for the micro climatic conditions in the chamber. Therefore, flux chambers are not necessarily representative for actual cow house conditions (Mosquera et al., 2008). As such they are not able to estimate the emission of a complete cow house, but may be useful for comparisons between housing systems in relative terms (Mosquera et al., 2010).

To summarise, the $\mathrm{NH}_{3}$ emission can be determined, but there are some challenges. $\mathrm{NH}_{3}$ emissions at farm level vary in space between different farms and within one farm, and time between seasons, months, days and hours. The emission is also influenced by many factors such as housing type and design, farm management, cow breed, diet, climate, etc. Therefore, to determine a standardised emission from a certain housing system in practice, a measurement protocol was developed (Ogink et al., 2013). This Dutch protocol is highly in accordance with the VERA protocol for North-West Europe. It prescribes the full-scale measurements of at least four identical cow houses for 6 times spread over a period of a year for at least $48 \mathrm{~h}$ per measurement. Recently, quite some $\mathrm{NH}_{3}$ mitigation methods became available and were applied in Dutch dairy cow houses. For regulatory purposes emission factors have to be assigned to these new methods, based on the protocol. To comply with this requirement, a lot of full-scale dairy cow houses measurements have to be performed. These measurements takes a substantial amount of time, and consequently they are expensive. In addition, full-scale measurements can only be applied in a completely built and occupied cow house, which makes the whole process slow to find up-to-date $\mathrm{NH}_{3}$ emission information. For more details about the Dutch $\mathrm{NH}_{3}$ emission status quo, see Section 1.5.

Another challenge is the accuracy and precision. The problem is that current low cost methods to assess the $\mathrm{NH}_{3}$ emission have a low accuracy and precision, whereas intensive measurements with advanced equipment are quite good, but are more expensive and not always practically feasible. The costs to measure a single dairy cow house according to the protocol, with the current applied methods, is about $€ 10000$ to $€ 20000$, which is substantial. In addition, there is a large variation in types, designs and management of dairy cow houses in The Netherlands. This makes it more difficult to obtain reliable measurement results. Finally, the currently used full-scale cow house measurement methods do not include the measurement of variables related to the $\mathrm{NH}_{3}$ emission process (Section 1.2). Therefore, it is difficult to correctly match the full-scale measurements with theory and consequently also to identify opportunities to lower the $\mathrm{NH}_{3}$ emission. Hence, there is a need for a novel assessment strategy to determine the $\mathrm{NH}_{3}$ emission from commercial dairy cow houses at a yearly basis that is:

1. not too expensive $(<€ 10000)$;

2. fast i. e. low number of measurement days and low time to build or install the measurement equipment; 
3. accurate and precise;

4. simple to apply;

5. supports the known $\mathrm{NH}_{3}$ emission process theory (Section 1.2).

\section{$1.2 \quad \mathrm{NH}_{3}$ emission process}

The $\mathrm{NH}_{3}$ emission process and the related equations are described in detail in (Monteny \& Erisman, 1998) and many others, for example (Ni, 1999; Montes et al., 2009; Aarnink \& Elzing, 1998; Cortus et al., 2008; Elzing \& Monteny, 1997; Muck \& Steenhuis, 1981; Hashimoto, 1972), and it is also concisely described in Chapter 2 of this thesis.

In short. The $\mathrm{NH}_{3}$ converts from urea present in urine, catalysed by the enzyme urease, which is abundantly present in faeces (Ketelaars \& Rap, 1994). In other words, the urease activity controls the conversion speed, but in current practice this speed is not limited at all. In a liquid environment, like a urine puddle on a floor, the volatile $\mathrm{NH}_{3}$ equilibrates with the non-volatile $\mathrm{NH}_{4}^{+}$. The amount of $\mathrm{NH}_{3}$ depends on the acid dissociation constant for $\mathrm{NH}_{3}$ and the $\mathrm{pH}$. The $\mathrm{pH}$-effect is pronounced. Below a $\mathrm{pH}$ value of about 6 to 7 there is mainly $\mathrm{NH}_{4}^{+}$while above 7 the $\mathrm{NH}_{3}$ part increases, and above about 11 there is mainly $\mathrm{NH}_{3}$ (Monteny \& Erisman, 1998). The $\mathrm{NH}_{3}$ emission process, and the related $\mathrm{CO}_{2}$ emission process, in itself influence the $\mathrm{pH}$ level in a urine puddle (Blanes-Vidal et al., 2009; Chaoui et al., 2009; Hafner et al., 2013) and these processes are dynamic. The amount of volatile $\mathrm{NH}_{3}$ depends on the Henry's law constant, which describes the equilibrium between $\mathrm{NH}_{3}$ in liquid and gas form. Finally, the volatilisation of $\mathrm{NH}_{3}$ into the air is based on the convective mass transfer coefficient, and the concentration difference between the boundary layer of the source and the air above this source. The whole process is temperature related, at higher temperatures the process is faster. Besides temperature, the mass transfer coefficient is also related to the local air velocity and the emitting surface area. The total potential amount of $\mathrm{NH}_{3}$ of a certain source depends on the urea concentration and the volume of that source.

Despite the fact that the physical and chemical processes in the $\mathrm{NH}_{3}$ emission from dairy cow houses are well known, a couple of challenges exist. First the process parameters, being the acid dissociation constant, Henry's law constant and the mass transfer coefficient, are empirically determined and consequently a large range of "truth" equations were determined and they vary considerably (Ni, 1999; Montes et al., 2009). See Chapter two for a complete list of empirical equations per process parameter. Related to this variation, the relative and absolute importance of each parameter and related input variables to estimate the $\mathrm{NH}_{3}$ emission are unknown. In addition, it is not clear how to describe the emission process in the pit and determine its contribution to total emission (Ye et al., 2009; Wu et al., 2012). 


\section{3 $\quad \mathrm{NH}_{3}$ emission modelling}

Various mechanistic models that estimate the $\mathrm{NH}_{3}$ emission from livestock houses have been built, developed and validated in the past 25 years (Ni, 1999; Montes et al., 2009; Aarnink \& Elzing, 1998; Cortus et al., 2008; Elzing \& Monteny, 1997; Muck \& Steenhuis, 1981), based on the theory reported in the previous Section 1.2

In The Netherlands, an emission model was developed in the nineties of the last century (Monteny et al., 1998). This model is currently in use to pre-assess $\mathrm{NH}_{3}$ emission reduction of new mitigation methods, and is here referred to as the Tac-Rav model (see Section 1.5). The Tac-Rav model simulates a full dairy cow house by randomly distributing urinations of dairy cows in time and space. For each urine puddle the $\mathrm{NH}_{3}$ volatilisation process starts at "urination", and the emission of the whole house is the sum of the individual emitting puddles calculated per time step. The Tac-Rav model is dynamic with respect to the urea conversion, but static with respect to all the input variables and the other processes. The model is of course a simplification of reality; the 9 most important assumptions in this respect are discussed here:

1. During a simulation, all input variables are static, assumed to be constant in time.

2. The pit is a continuous $\mathrm{NH}_{3}$ emission source, which emits a constant amount of $\mathrm{NH}_{3}$ per $\mathrm{m}^{2}$ per time unit. This source is independent of the urine puddles on the floor and only dependent of the model parameters and input variables set at the start of a simulation. According to (Muck, 1982) a slurry storage emits continuously, but with a variable rate, depending on $\mathrm{pH}$, temperature and loading rate. Moreover, measures to reduce the emission of $\mathrm{NH}_{3}$ from the pit by (partly) closed floors cannot be simulated.

3. A urine puddle can only be replaced completely by a new one, partial replacement is not assumed and not possible.

4. As a consequence, the number of locations where a urine puddle can be dropped depends on the area covered by one puddle. The number of locations is calculated by total available floor area divided by the floor area covered by one urine puddle.

5. The dimensions of a urine puddle do not change in time. There is no evaporation of water from the urine puddle, only $\mathrm{NH}_{3}$ emission.

6. Incoming air, flowing over the puddles and slurry in the pit, does not contain $\mathrm{NH}_{3}$.

7. Related to 6 , the $\mathrm{NH}_{3}$ that emits from a puddle is directly removed in the next time step. Especially in the pit, however, when partially closed, the air will contain $\mathrm{NH}_{3}$ in a next time step (Wu et al., 2012). 
8. The total nitrogen balance of the cow house (N-balance) is not modelled, possibly leading to unrealistic emission rates.

9. Currently, input variable values are not measured (Sections 1.1 and 1.5). Therefore, it is difficult and arbitrary to define input variable values to simulate a cow house with one or more $\mathrm{NH}_{3}$ emission mitigation methods.

(Wang et al., 2006) built a similar mechanistic model compared to the one of (Monteny et al., 1998). Compared to practical measured data, the overall results of the models of both Wang and Monteny fitted the data well with a $11 \%$ and $7 \%$ higher model estimated $\mathrm{NH}_{3}$ emission respectively (Wang et al., 2006). However, no verification or validation was performed with respect to the contribution of the emitting sources, the physical and chemical processes, and the involved input variables and model parameters. In addition, to simulate and predict the $\mathrm{NH}_{3}$ emission from commercial dairy cow houses, the simplified mechanistic models need adequate values for the model parameters and the input variables. However, these are not measured or estimated from real-time data in current measurement practice (Sections 1.1 and 1.5). Besides, the importance of them is unknown (Section 1.2).

An alternative type of modelling is Computational Fluid Dynamics (CFD). CFD is a design and analysis tool to numerically solve fluid flow, heat and mass transfer (Ferziger \& Peric, 2002). With CFD it is possible to build a physical system in a computational model to study different operational conditions and design constraints. CFD consist of mainly three parts: pre-processing, solving and post-processing (Norton et al., 2007): "The pre-processing environment includes problem consideration, geometry creation, mesh development, physical property set-up, and implementation of solving techniques and parameters.". The solver puts the pre-processor information into numerical arrays and solves them iteratively. Finally, the post-processing environment allows the user to visualise and scrutinise the resulting field solution (Norton et al., 2007). In the case of $\mathrm{NH}_{3}$ emission from dairy cow houses, CFD can be used to predict the air flow direction and velocity, and air characteristics like gas concentrations or temperature in all distinguished space compartments (e.g. $1 \mathrm{~cm} \times 1 \mathrm{~cm} \times 1 \mathrm{~cm}$ ) of the complete house. Hence, the air speed and temperature close to the $\mathrm{NH}_{3}$ sources, and the transport of $\mathrm{NH}_{3}$ from the source to the outlets can be found. However, CFD cannot simulate at individual puddle level. The processes related to urea conversion, $\mathrm{NH}_{3}$ equilibrium and evaporation has to be modelled as in mechanistic models. An important limitation of CFD is that its accuracy is limited by uncertainties in specifying the boundary conditions. Boundary conditions were based on information of the surroundings of the modelled physical system. There is a lack of understanding the surroundings of an object and to quantify turbulent air motion. As a result of computational limitations CFD models cannot contain microscopic details and simplification is needed. The same holds for the time step of calculations. Optimal time stepping is a trade-off 
between computational efficiency, temporal accuracy, and stability of the numerical scene (Norton et al., 2007). In addition, experimental validation with detailed data is necessary (Norton et al., 2007), which is difficult to obtain in full-scale dairy cow houses (Section 1.1).

The Dutch NEMA model (Nationaal Emissie Model Ammoniak; Velthof et al., 2012 ) is a $\mathrm{N}$-balance or flow based model that calculates the $\mathrm{NH}_{3}$ emission from all livestock species for all involved sources like housing, storage, application, and pasturing. NEMA uses mean or generic values for so-called emission factors, being the relative amount of $\mathrm{N}$ emitted as $\mathrm{NH}_{3}$ for existing techniques and management practices. This model is not meant to estimate emission rates from specific sources or situations, nor from new reduction measures.

Research has been done to assess the effect of feeding strategies on the $\mathrm{NH}_{3}$ emission. The relation between the urea-nitrogen concentration in the milk (MUN) and the emission of $\mathrm{NH}_{3}$ from dairy cow houses was measured and modelled by means of black-box statistical models (Burgos et al., 2005, 2007, 2010; Monteny et al., 2002; van Duinkerken et al., 2003, 2005, 2011). The results however, do not always agree, and they are obtained from studies with adjusted diets that not necessarily represent practice. The relation between diet, MUN and urea-nitrogen in the urine (UUN) was also investigated, but this was only performed at dairy cow level (Spek et al., 2012a,b, 2013a,b).

Despite that various modelling approaches and models for the $\mathrm{NH}_{3}$ emission of dairy cow houses exist, none of them can be used straight away for the purpose to assess the actual emission from a commercial dairy cow house precisely, either conventional or equipped with mitigation methods. Current mechanistic $\mathrm{NH}_{3}$ emission models like the Tac-Rav model are too simplified and involved processes are not sufficiently validated. CFD models, on the other hand, are complex and need a lot of detailed information on configuration of the house layout, and emission rate of the sources still has to be modelled as with mechanistic models. The situation of a commercial dairy cow house is basically too complex to model with CFD and to validate it. To summarise, we lack an adequate model that is sufficiently flexible to be able to cover a wide range of practical dairy cow houses, and that also connects with measurements.

\section{$1.4 \mathrm{NH}_{3}$ emission mitigation methods}

Solutions have been developed to reduce the $\mathrm{NH}_{3}$ emission from dairy cow houses. They can be summarized by policy instruments that are categorized according to their pollution swapping potential (Oenema et al., 2007):

1. Mitigation or abatement of $\mathrm{N}$ species emissions;

2. Controlling $\mathrm{N}$ input (diet);

3. Extensification of agricultural production and environmental protection; 
4. Regulations on animal welfare;

5. Improving the competitiveness of agricultural sectors;

6. Spatial zoning.

To reduce the NH3 emission through the design of dairy cow houses (category 1) different measures can be taken by the Best Available Technique (BAT) (Oenema et al., 2007; Monteny \& Erisman, 1998):

- Cow house adaptation by improved design and construction of the floor;

- Dilution or removal of urine from floors;

- Separation of urine and faeces on the floors;

- Cover slurry storage and reduce or eliminate the air exchange between slurry pit and house;

- Bio-filtration of outgoing air (end of pipe technique);

- Reduce urea concentration in the urine of cows for example by means of feeding measures;

- Slowing down the urea hydrolysis in urine puddles and the slurry pit;

- Control of $\mathrm{pH}$ in the urine puddles;

- Reduce the mass transfer of $\mathrm{NH}_{3}$.

Several methods have been developed to reduce the $\mathrm{NH}_{3}$ emission from dairy cow houses (van Dooren \& Smits, 2007). New floors have been investigated and compared to the commonly used slatted floor. Floors can cover the pit partially or completely and they transport or even separate urine and faeces. First, there are new concrete floor, like solid floors with or without slope or gutter(s) (Swierstra et al., 1995; Braam \& van den Hoorn, 1996; Braam et al., 1997a,b) and grooved floors (Swierstra et al., 1997, 2001). Recently, floors with all kind of profiles with or without sloped gutters and pit closure methods became available (Tac-Rav, 2016) Reduction effects of these concrete floors are expected and measurements are currently been carried out to assess the effect under practical circumstances, according to the defined protocol (Section 1.1; Ogink et al., 2013). In addition, new types of freestall cow house designs including new types of floors are under development (van Dooren \& Galama, 2009). E.g. complete free floors, without cubicles or other obstructions, consisting of loose bedding material that mixes with urine and faeces, like compost, sand, wood shavings, dry manure, straw, or a top layer of rubber without loose material. The new designs have a positive effect on the welfare of the cows, but emissions of $\mathrm{NH}_{3}$ and other gases like methane and nitrous oxide tend to be higher than from conventional slatted floor systems with a slurry pit underneath (Smits et al., 2009).

Manure scrapers have been installed frequently over the last years in The Netherlands. They clean the concrete floor by pushing faeces and urine to openings where 
it can fall down into the slurry pit. The cleaning affect may result in a lower $\mathrm{NH}_{3}$ emission, but scraping frequency, scraper design, and floor type are at least influencing factors (Braam et al., 1997a). Two types of scrapers can be distinguished: 1) a conventional scraper fixed to the cow house that is guided and pulled; 2) a robot with a small scraper with either a fixed or random path (Blanken, 2007).

Other methods investigated are flushing and dilution with water, acidifying slurry to lower $\mathrm{pH}$, use of urease inhibitors to limit urea conversion, cooling of the floor and slurry, and reduction of ventilation rate (Braam \& Swierstra, 1999; Scholtens et al., 1996; Ogink \& Kroodsma, 1996; Varel et al., 2007; Parker et al., 2005; Leinker, 2007; Hagenkamp-Korth et al., 2015a,b). Until now urease inhibitors are not recognised as a BAT to reduce emissions in practise, but floor flushing with water is available in combination with a manure scrape robot (Zevenbergen, 2009).

As described, many methods, techniques, and management or feeding strategies have been developed to reduce the emission of $\mathrm{NH}_{3}$ from dairy cow houses and probably the list will even not be complete. However, the exact extent of the emission reduction under commercial conditions is not clear for all these measures. This may be caused by the measurement costs and the lack of cheap methods to assess the emission on the one hand (Section 1.1). On the other hand, however, it is difficult to distinguish the reduction effect of a single measure within the setting of a commercial dairy cow house. Measurements in the lab may be the solution, but in the lab there are controlled conditions, which may not be realistic. In other words, positive emission reduction results in the lab will not necessarily lead to reduction in practice. Therefore, there is a need for a method that can determine the effect of various kinds of $\mathrm{NH}_{3}$ reduction measures effectively and with limited costs, and distinguish these measures from random variation and other influencing factors.

\section{5 $\mathrm{NH}_{3}$ emission reduction approach - status quo in The Netherlands}

To reduce the $\mathrm{NH}_{3}$ emission from dairy cow houses, farmers have to apply $\mathrm{NH}_{3}$ emission mitigation methods in new dairy cow houses. Available methods are on a list in the 'Regulation on $\mathrm{NH}_{3}$ from livestock production', coded Rav (Tac-Rav, 2016). New proposed methods for the Rav-list are pre-assessed by the governmental Technical Advisory Committee (Tac-Rav). Experts in the Tac-Rav use the $\mathrm{NH}_{3}$ emission model developed by (Monteny et al., 1998) (Section 1.3), to carry out pre-assessments of new low-emission cow houses. They estimate the effects of the applied mitigation method, the floor design and management characteristics, and consequently the model is used to calculate the $\mathrm{NH}_{3}$ emission from the floor area and slurry pit. Based on this pre-assessment, provisional emission factors are assigned to the applied mitigation methods, that are later replaced by definite emission factors after $\mathrm{NH}_{3}$ emissions have 
been measured in practice according to the prescribed protocol (Ogink et al., 2013). As described in Section 1.1, the protocol results in a large number of full-scale dairy cow house measurements, which takes a lot of time and is expensive. Therefore, the current procedure causes a long delay before an initial idea of a new $\mathrm{NH}_{3}$ emission reduction method is tested, approved and available in practice. Subsequently, this long delay causes uncertainty for all involved parties.

The so-called Tac-Rav model used in the pre-assessment phase was originally developed for research purposes. The Tac-Rav model requires estimates of a large number of variables that should be representative for practical conditions. However, the currently available information is not sufficient to arrive at accurate estimates of input values that reflect floor and management characteristics of new housing designs. Besides, the model variables are not part of the currently applied protocol (Ogink et al., 2013). The scarcity of information is mainly caused by the complexity to measure required floor and manure storage variables under real cow house conditions. The little that is known is mainly derived from research carried out before 2000, under cow house and management conditions this may have changed since then. In the process of pre-assessment there is a need for a simpler, more pragmatic emission model, i. e. with less input variables, and a need for actual measurement data of these input variables from dairy cow houses reflecting current practical conditions.

\subsection{Objectives and Thesis outline}

The focus of this $\mathrm{PhD}$ thesis is on the $\mathrm{NH}_{3}$ emission from fresh urine puddles in dairy cow houses. The overall objective of the thesis is:

\section{Refining a model-based assessment strategy to estimate the ammonia emission from floors in dairy cow houses}

The overall objective was split up in four sub-objectives:

1. Identify the most important input variables and process parameters in current available mechanistic $\mathrm{NH}_{3}$ emission models and theory.

2. Explore, identify, develop, and improve sensors and measurement methods for these most important input variables to measure them in commercial dairy cow houses.

3. Assess the values and interactions of the identified input variables for fresh urine puddles in commercial dairy cow houses.

4. Study the effect of these input variable values of fresh urine puddles on the estimated $\mathrm{NH}_{3}$ emission. 
These objectives are worked out in detail in seven chapters. In Chapter 2 all current available mechanistic $\mathrm{NH}_{3}$ emission models were described and analysed in a global sensitivity analysis. From this research the most important urine puddle related input variables were identified and it was observed that there was hardly data available. Data was scarce because of the lack of appropriate measurement methods, and the difficulty to perform measurements among cows. Therefore, sensors were explored and measurement methods were developed and tested in Chapters 3, 4, and 5 .

In Chapter 3 both a pH sensor and a temperature sensor were selected that were able to measure the $\mathrm{pH}$ and temperature of thin fluid layers, like urine puddles on floors. In addition, in total 26 fresh dairy cow urine puddles in three commercial dairy cow houses were measured in $15000 \mathrm{~s}$ time series to investigate $\mathrm{pH}$ values and to study their dynamic behaviour. Chapters 4 and 5 describe the measurement methods that were developed and tested in the lab to ultimately obtain the puddle depth (Chapter 4) and puddle area (Chapter 5) values from urine puddles in commercial dairy cow houses. Furthermore, the sensor performance was monitored.

Then in Chapters 6 and 7 all developed measurement methods and sensors were combined in one measurement series to obtain the puddle area \& depth (Chapter 6 ), and the puddle $\mathrm{pH}$, urea concentration \& temperature values (Chapter 7) simultaneously of fresh urine puddles on floors in 16 commercial dairy cow houses. The 16 houses were divided up into a factorial setup of four groups of four based on floor type and farm management.

Finally, Chapter $\boldsymbol{8}$ comprises a general discussion including concluding remarks and recommendations. 
Sensitivity analysis of mechanistic models for estimating ammonia emission from dairy cow urine puddles

Dennis Snoek

Hans Stigter

Nico Ogink

Peter Groot Koerkamp

Published in Biosystems Engineering (2014), 121, 12-24 


\begin{abstract}
Ammonia $\left(\mathrm{NH}_{3}\right)$ emission can cause acidification and eutrophication of the environment, is an indirect source of nitrous oxide, and is a precursor of fine dust. The current mechanistic $\mathrm{NH}_{3}$ emission base model for explaining and predicting $\mathrm{NH}_{3}$ emissions from dairy cow houses with cubicles, a floor and slurry pit is based on measured data from a limited number of studies. It requires input values for numerous variables, but the empirical equations for the model parameters in the literature vary. Furthermore, many of the input variables cannot be assessed accurately, and their actual influence on the prediction is unknown. We aimed to improve $\mathrm{NH}_{3}$ emission modelling, by assessing the contribution to the variation in $\mathrm{NH}_{3}$ emission of each input variable and each model parameter related to a single urine puddle. We did so for 27 candidate models, created by each possible combination of three equations per model parameter: the acid dissociation constant, Henry's law constant, and the mass transfer coefficient. After analysing each candidate model with a Global Sensitivity Analysis we found that at least $71 \%$ of the model variation in $\mathrm{NH}_{3}$ emission for each candidate model was explained by five puddle related input variables: $\mathrm{pH}$, depth, area, initial urea concentration, and temperature. $\mathrm{NH}_{3}$ emission was not sensitive for the other four variables: air temperature, air velocity, maximum rate of urea conversion, and Michaelis-Menten constant for urea conversion. Based on these results we recommend simplifying the model structurally and reducing the number of input variables.
\end{abstract}




\subsection{Introduction}

Ammonia $\left(\mathrm{NH}_{3}\right)$ emission can cause acidification and eutrophication of the environment. $\mathrm{NH}_{3}$ is also an indirect source of the greenhouse gas nitrous oxide $\left(\mathrm{N}_{2} \mathrm{O}\right)$ (IPCC, 1996) and is a precursor of fine dust particles. To lower $\mathrm{NH}_{3}$ emissions in the EU, member states are required to set a National Emission Ceiling (NEC) (UNECE, 1999; EU, 2001). Twenty-five of the 27 EU member states complied with the 2010 NEC set by the European Commission. The total emission of $\mathrm{NH}_{3}$ in the Netherlands in that year was $122 \mathrm{kt}$, which was $4.9 \%$ below NEC 2010 (EEA, 2012). However, local and regional emission and deposition still cause a high overload in the Dutch Natura2000 areas (PBL, 2012). If, as expected, the NECs set for 2020 are lower than those set for NEC 2010, further mitigation of $\mathrm{NH}_{3}$ emission will be necessary in the EU.

In 2010, agriculture was responsible for $94 \%$ of all $\mathrm{NH}_{3}$ emission from the $27 \mathrm{EU}$ member states (EEA, 2012). Of this, $80 \%$ was emitted from livestock production systems. $\mathrm{NH}_{3}$ Emission from cattle in the Netherlands fell from $184 \mathrm{kt}$ in 1990 to 53 kt in 2009 (van Bruggen et al., 2011), of which $34 \%$ originated from dairy cow houses and manure storage facilities. Monteny et al. (1998) considered a typical dairy cow house consisting of a living area with cubicles, plus walking and feeding-alleys, which together provide a total area of $3.5 \mathrm{~m}^{2}$ per cow. There is a slurry pit underneath the whole house, and a slatted, concrete floor in the cow walking area. They estimated that one urine puddle occupies an area of $0.8 \mathrm{~m}^{2}$. In such a typical dairy cow house about $70 \%$ of $\mathrm{NH}_{3}$ emission is emitted from the slatted floor.

Monteny et al. (1998) developed a conceptual mechanistic computer model in order to understand and predict $\mathrm{NH}_{3}$ emissions from dairy cow houses. Called the Monteny model, it describes the physical and chemical processes involved and quantitatively determines the $\mathrm{NH}_{3}$ emission according to model parameters, using input variables related to characteristics of a urine puddle, air, floor and pit. Similar mechanistic models have been developed and validated against measurements in a limited number of studies for cows (Muck \& Steenhuis, 1981; Elzing \& Monteny, 1997; Montes et al., 2009; Vaddella et al., 2011, 2013), and for pigs (Zhang et al., 1994; Aarnink \& Elzing, 1998; Arogo et al., 1999; Liang et al., 2002; Cortus et al., 2008). In this study we focus on the general mechanistic $\mathrm{NH}_{3}$ emission model theory.

The Monteny model is currently used by the Dutch Ministry of Infrastructure and Environment to assess $\mathrm{NH}_{3}$ emission of dairy cow houses that are applying new $\mathrm{NH}_{3}$ mitigation techniques, and also to obtain preliminary emission factors that are used when granting permits. This assessment is later followed by full-scale measurements in commercial houses in accordance with a prescribed protocol, with the aim of establishing definite emission factors (Ogink et al., 2011). Determining emission factors in commercial houses using full-scale measurements is costly and time consuming. The accuracy of the results is also an issue, as emissions vary greatly 





between countries, animal houses and seasons (Groot Koerkamp et al., 1998). There is a need for an $\mathrm{NH}_{3}$ emission model that would support, simplify and standardise current measurement methods, and help elucidate and explain the emissions measured and their variations. This would benefit research in $\mathrm{NH}_{3}$ mitigation technology and the testing of new housing designs. The model should be as simple as possible, but still fit for purpose. Current mechanistic $\mathrm{NH}_{3}$ emission models require input values for numerous variables, but various empirical equations for model parameters have been reported in the literature and many of the input variables cannot be measured or assessed accurately in practice and their actual influence on the emission is unknown.

When Monteny et al. performed a limited one-factor-at-a-time sensitivity analysis of their model (Monteny et al., 1998), they did not include all input variables. Because the values of input variables can vary widely in practice, the empirical equations for the model parameters vary $(\mathrm{Ni}, 1999)$ and variables can interact, it remains unclear to which variables the model is most sensitive.

Our objective was to gain knowledge to improve $\mathrm{NH}_{3}$ emission models. To do so, our first step was to review the literature on emission models in order to ascertain the relevance of the input variables, model parameters, and model structure. In this study we assessed the contribution to the variation in $\mathrm{NH}_{3}$ emission of each input variable and each model parameter in the mechanistic $\mathrm{NH}_{3}$ emission model of a single urine puddle on a floor.

\subsection{Materials and Methods}

In Section 2.2.1 - 2.2.5 we describe the general emission model. Each equation for model parameters was converted to standardised units for comparison. From this comparison three equations per model parameter were selected and used in each possible combination, in order to create candidate models. This yielded 27 candidate models. For each candidate model and for each input variable, we determined the first- and second-order sensitivity coefficients, and the total effect index, as described in Section 2.2.6. This was done for the absolute $\mathrm{NH}_{3}$ emission of a single urine puddle on a floor, for three emission durations (ed), described in Section 2.2.7.

$\mathrm{NH}_{3}$ emission is here defined as the process whereby $\mathrm{NH}_{3}$ is emitted from urine, faeces, or slurry into the air. We focus on a urine puddle on the floor, produced by dairy cows. This process consist of four steps (Fig. 2.1):

1. Urea conversion to $\mathrm{NH}_{3, \text { liq. }}$.

2. Equilibrium between $\mathrm{NH}_{3, \text { liq }}$ and $\mathrm{NH}_{4}^{+}$, liq based on the dissociation constant.

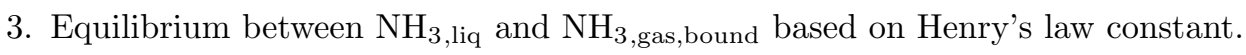

4. Volatilization into the air based on the convective mass transfer coefficient. 


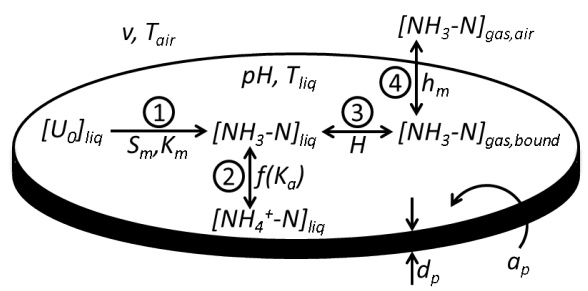

Figure 2.1: Schematic representation of the ammonia emission process in a urine puddle. For abbreviations see Nomenclature, and for numbers see Section 2.2.

\subsubsection{Urea conversion}

Urea in a urine puddle converts to $\mathrm{NH}_{3}$ and $\mathrm{CO}_{2}$, Eqs. (2.1) to (2.3) catalysed by the enzyme urease (Muck, 1982; Elzing \& Monteny, 1997) that is present in faeces on the floor.

$$
\begin{gathered}
\mathrm{CO}\left(\mathrm{NH}_{2}\right)_{2}+\mathrm{H}_{2} \mathrm{O} \stackrel{\text { urease }}{\longrightarrow} 2 \mathrm{NH}_{3}+\mathrm{CO}_{2} \\
\frac{d[U]_{l i q}}{d t}=-\frac{S m \cdot[U]_{l i q}}{\mathrm{Km}+[U]_{l i q}} \\
{[U]_{l i q}(0)=\left[U_{0}\right]}
\end{gathered}
$$

Equation (2.2) is the well-known Michaelis-Menten equation with $S m$ the maximum rate of conversion, $K m$ the Michaelis-Menten constant, $[U]_{l i q}$ the concentration of nitrogen in urea (urea-N) in the urine, and $t$ the time. Different researchers have used different units to determine $S m$, resulting in different values (Table 2.1). To compare different values, we defined a standard unit for $S m$ as $m o m^{-3} \mathrm{~s}^{-1}$. Each $S m$ value in Table 2.1 was converted to this standardised unit. For $K m$ only Elzing \& Monteny (1997) determined a value (of $2.0 \mathrm{~mol} \mathrm{~m}^{-3}$ ) and this was used by Monteny et al. (1998), Aarnink \& Elzing (1998), and Cortus et al. (2008).

\subsubsection{Acid dissociation constant}

In the puddle, the $\mathrm{NH}_{3}$ formed equilibrates with $\mathrm{NH}_{4}^{+}$: Eq. (2.4). This equilibrium depends on the urine $p H$, and its temperature $\left(T_{l i q}\right)$ (Monteny et al., 1998).

$$
\mathrm{NH}_{3}+\mathrm{H}_{2} \mathrm{O} \stackrel{p H, T_{l i q}}{\longleftrightarrow} \mathrm{NH}_{4}^{+}+\mathrm{OH}^{-}
$$

To model the equilibrium process we used the acid dissociation constant $\left(K_{a}\right)$ : Eq. (2.5) (Hashimoto, 1972), and $p H$. Using $K_{a}$, and $p H$, the fraction $(f) \mathrm{NH}_{3}-\mathrm{N}$ was 
determined: Eq. (2.6) (Zhang et al., 1994).

$$
\begin{aligned}
& K_{a}=\frac{\left[N H_{3}\right]\left[H^{+}\right]}{\left[N H_{4}^{+}\right]} \\
& f=\frac{K_{a} \cdot 10^{p H}}{K_{a} \cdot 10^{p H}+1}
\end{aligned}
$$

There are major variations between studies in the empirical equations for $K_{a}$, as shown in Table 2.2. Figure 2.2 shows the dependence of $K_{a}$ on the temperature of the urine $\left(T_{l i q}\right)$. We observed that $K_{a}$ increased with increasing $T_{l i q}$ for each equation. At $T_{l i q}=35^{\circ} \mathrm{C}$ there was a ninefold difference between the equations.

\subsubsection{Henry's law constant}

To determine $\mathrm{NH}_{3}-\mathrm{N}_{\text {gas,bound }}$, Eq. (2.10), we used the Henry's law constant $(H)$, which describes the solubility of a gas in a liquid on the basis of the pressure of the gas. $H$ acts at the interface between the liquid phase and the gas phase. According to Lewis \& Whitman (1924), and Sander (1999) the Henry's law constant is:

$$
H=\frac{c_{l i q}}{p_{\text {gas }}}
$$

where $c_{l i q}$ is the concentration of a solute in the liquid phase and $p_{\text {gas }}$ is the partial pressure of that solute in the gas phase. The dimensionless form (Sander, 1999) is the ratio of $c_{l i q}$ to the concentration in the gas phase $\left(c_{g a s}\right)$ and is denoted by $H^{c c}$ in Eq. (2.8):

$$
H^{c c}=H \cdot R \cdot T_{l i q}=\frac{c_{l i q}}{c_{g a s}}
$$

where $R$ is the gas constant, and $T_{l i q}$ is the liquid temperature. To represent volatility the inverse definition can be used, Eq. (2.9) (Sander, 1999). To determine $\mathrm{NH}_{3}-\mathrm{N}_{\text {gas,bound }}, H_{i n v}^{c c}$ was used, Eq. (2.10) (Elzing \& Monteny, 1997).

$$
\begin{gathered}
H_{i n v}=\frac{1}{H}=\frac{p_{g a s}}{c_{l i q}}, \text { and } H_{i n v}^{c c}=\frac{1}{H^{c c}}=\frac{c_{g a s}}{c_{l i q}} \\
{\left[N H_{3}-N\right]_{g a s, \text { bound }}=f \cdot[T A N]_{l i q} \cdot H_{i n v}^{c c}}
\end{gathered}
$$

There are major variations between studies in the empirical equations for $H, H_{\text {inv }}$, $H^{c c}$, and $H_{i n v}^{c c}$, as shown in Table 2.3. Figure 2.3 shows the dependence of $H_{i n v}^{c c}$ on $T_{l i q}$. We observed that $H_{i n v}^{c c}$ increased with increasing $T_{l i q}$ for each equation. At $T_{l i q}=35{ }^{\circ} \mathrm{C}$ there was a twofold difference between the equations. 


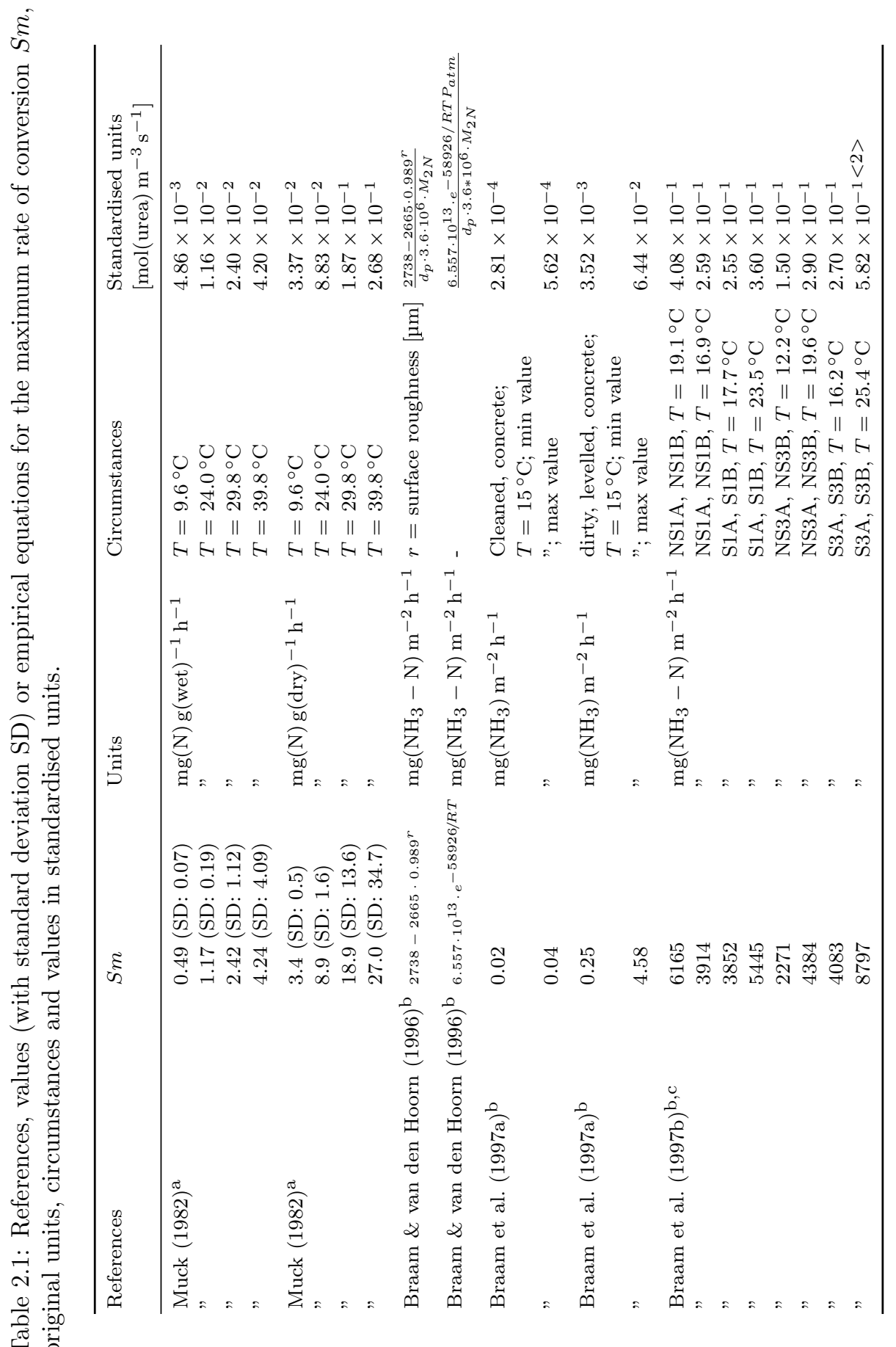




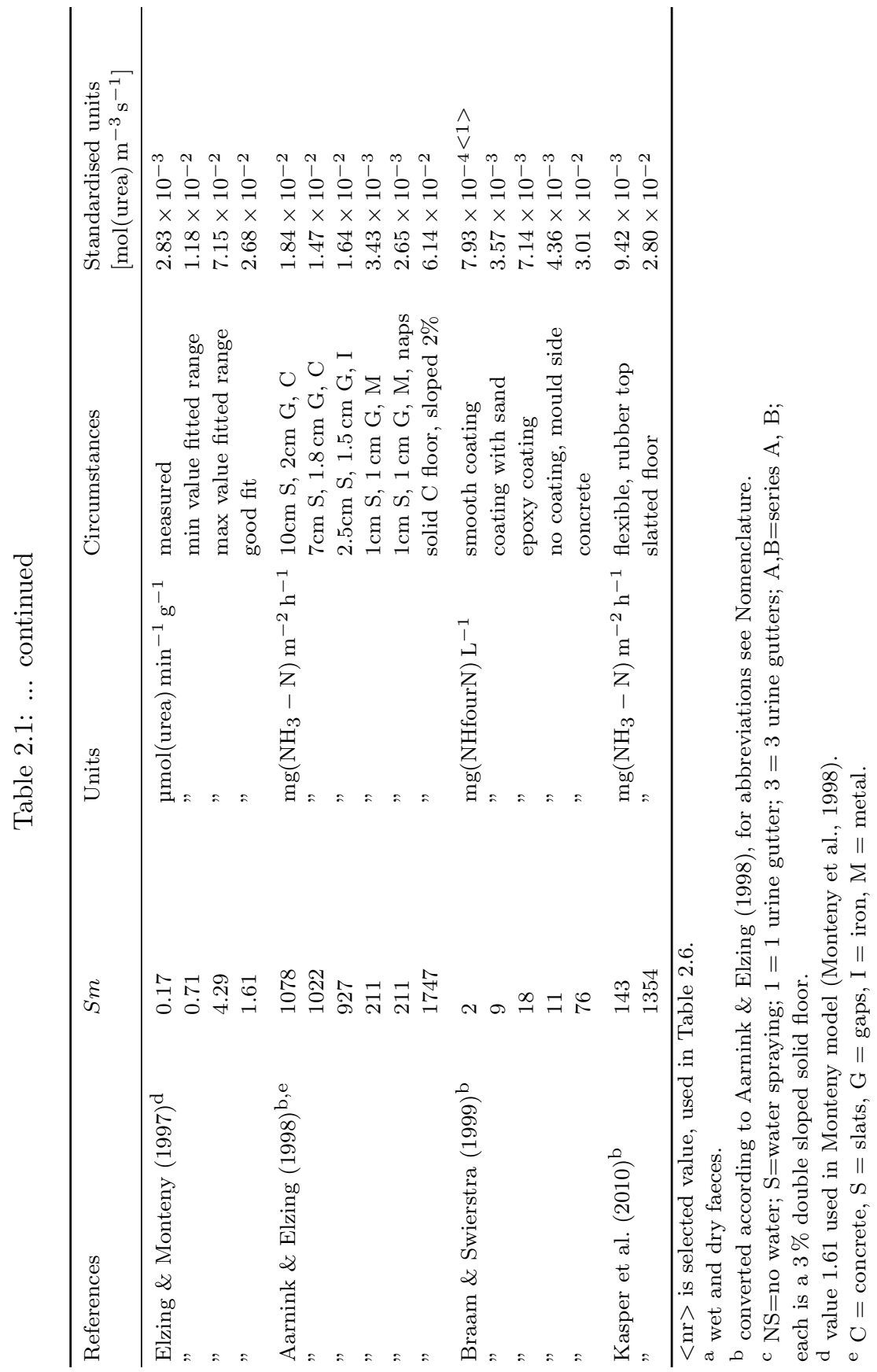




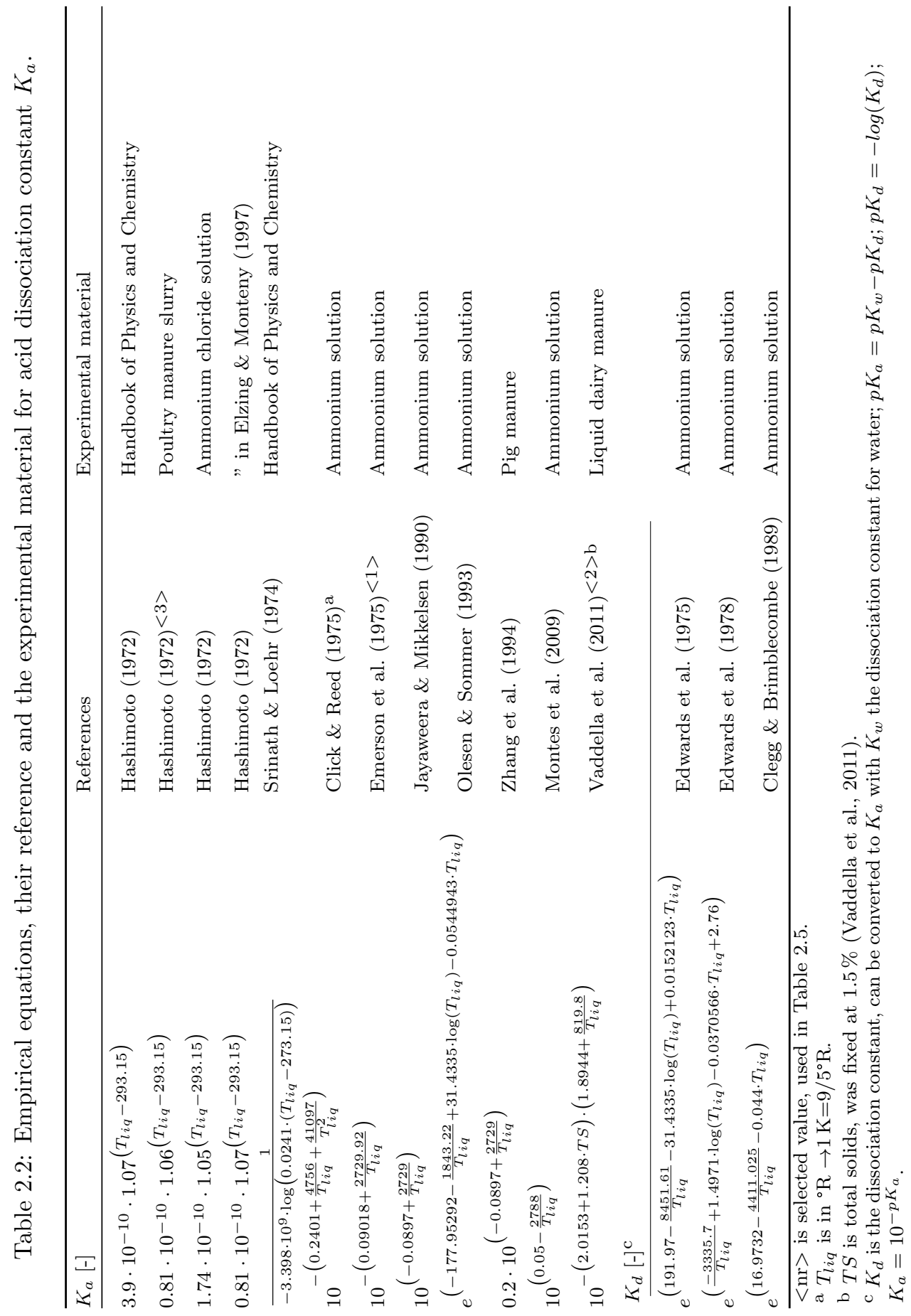




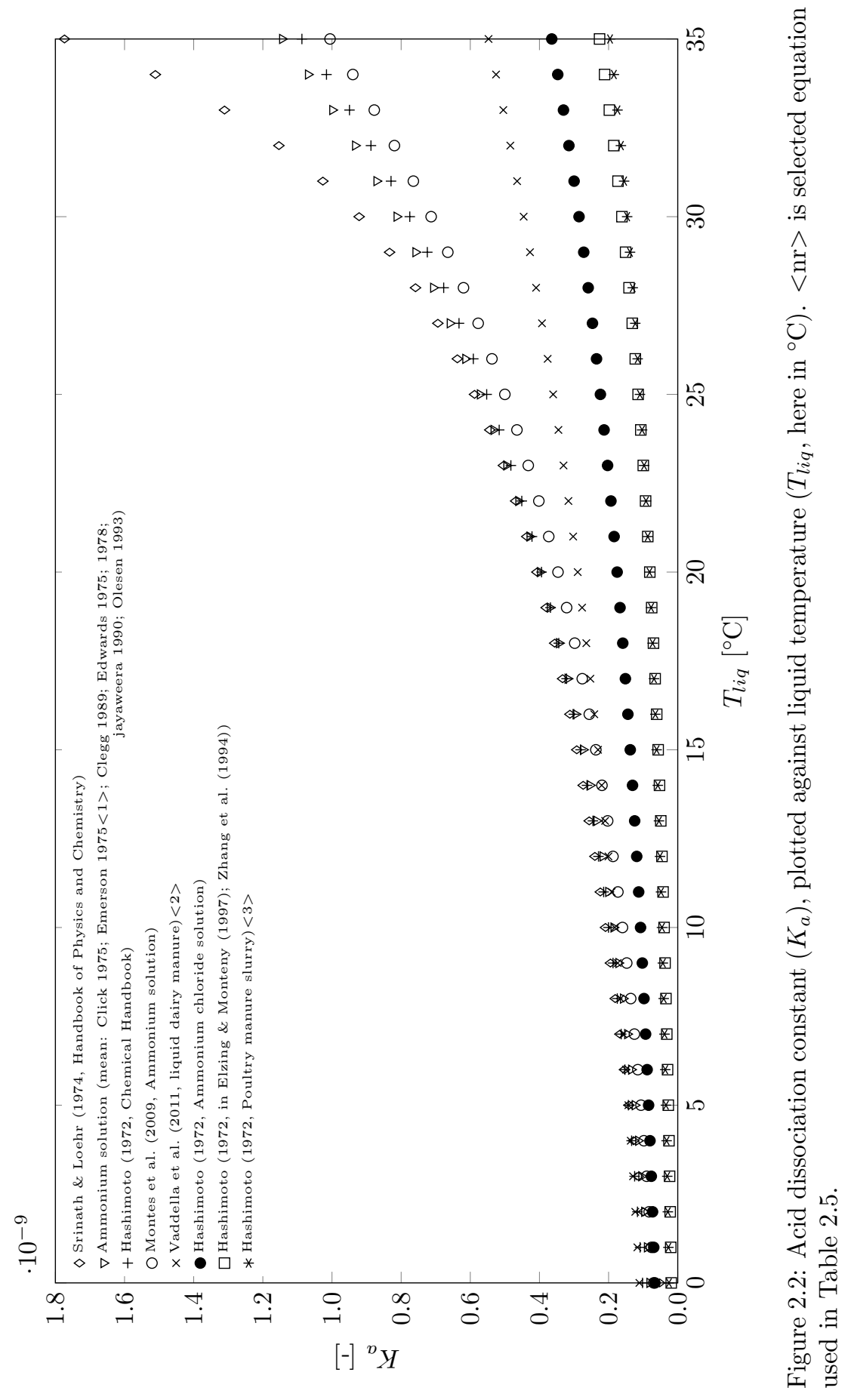




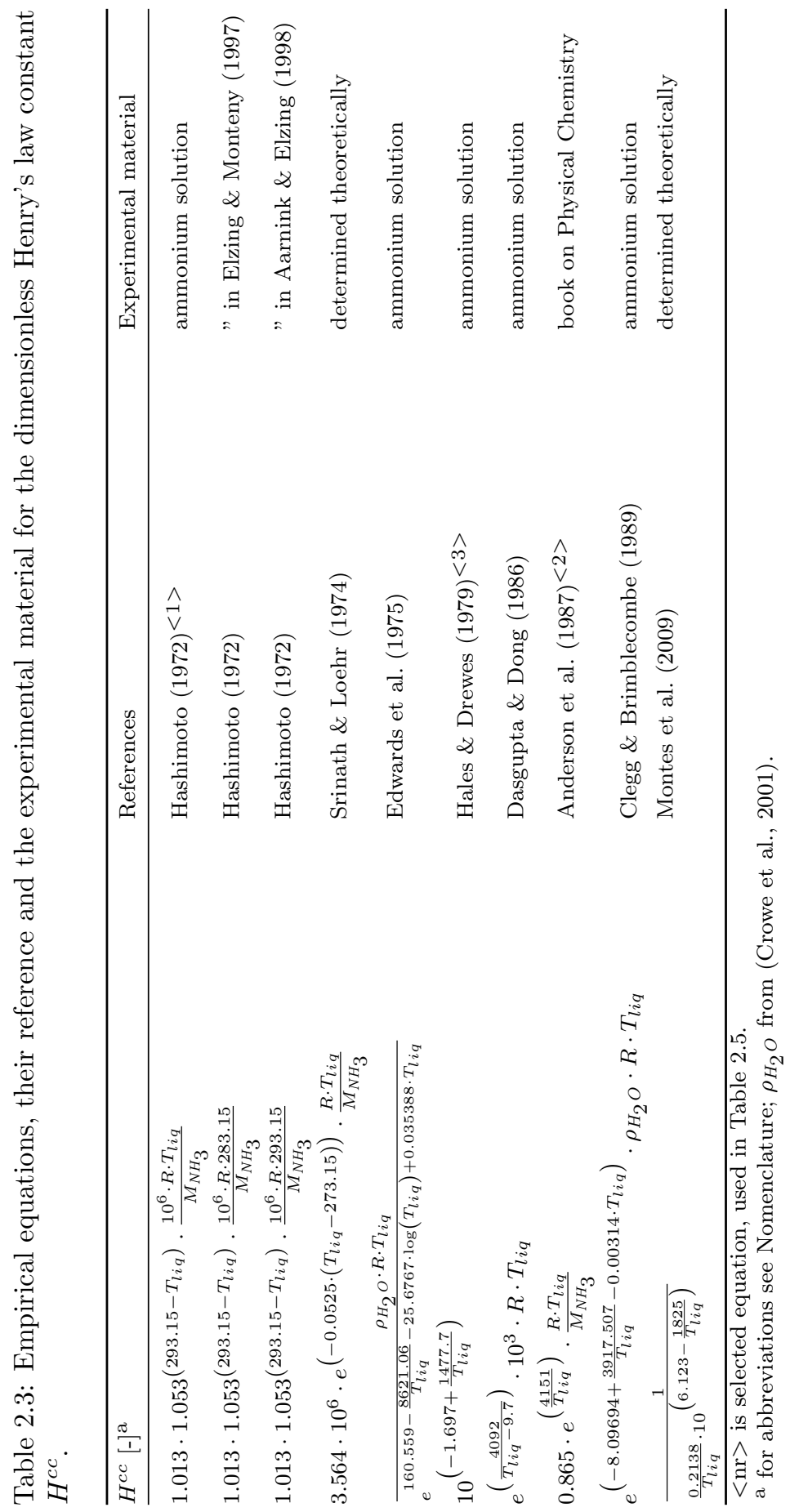




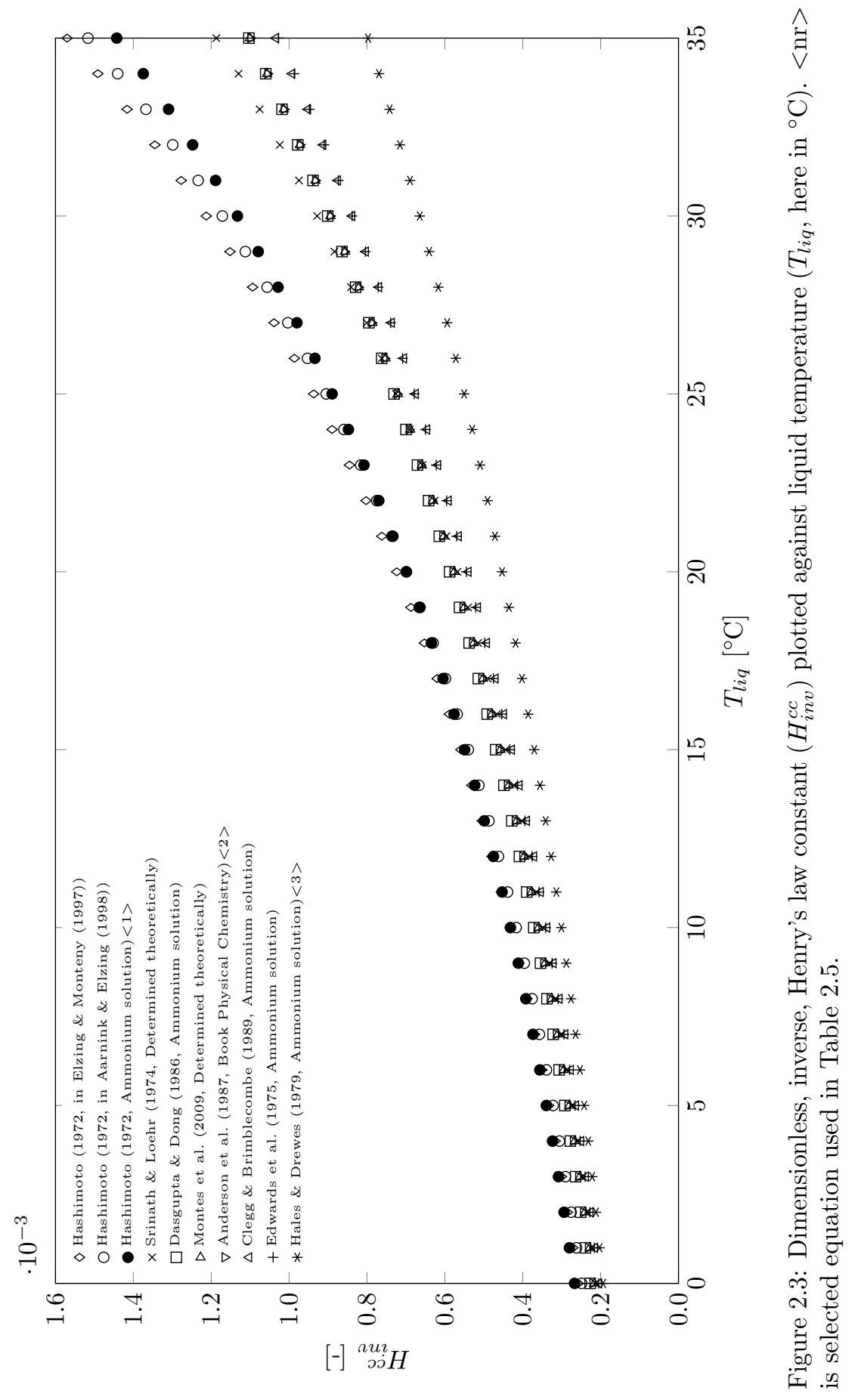




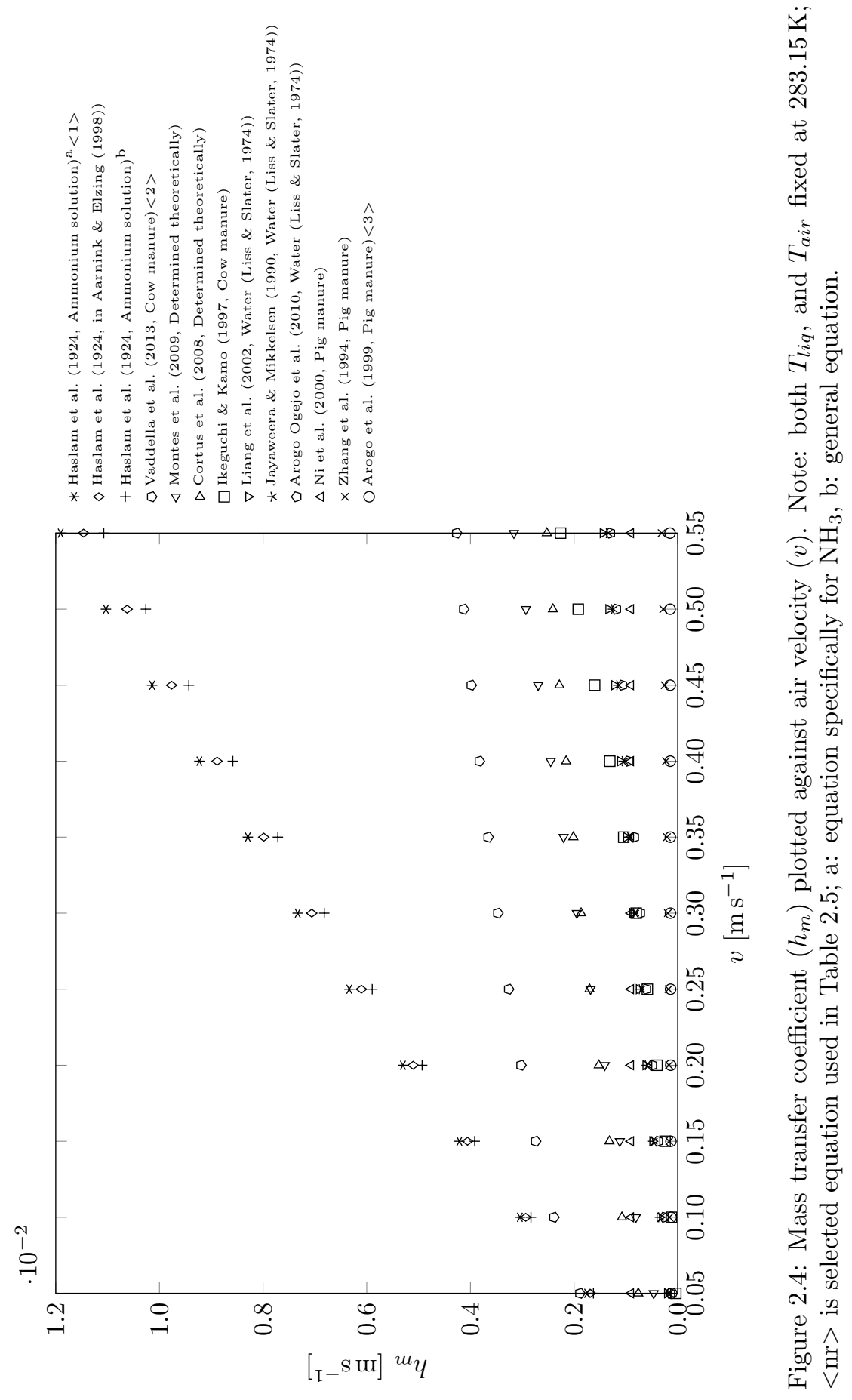




\subsubsection{Mass transfer coefficient}

As described by $\mathrm{Ni}$ (1999), two theories are used for the $\mathrm{NH}_{3}$ emission process, namely the "two-film theory" and the "boundary layer theory". The two-film theory assumes that the rate of diffusion through the liquid and gas films controls the rate of mass transfer, and that there is no resistance at the interface. The boundary layer theory assumes an $\mathrm{NH}_{3}$ concentration boundary layer between the puddle surface and the flow of air above. Characteristics of the boundary layer determine the mass transfer coefficient. Because most of the $\mathrm{NH}_{3}$ emission models for puddles, waste lagoons and slurry or manure storage facilities use the two-film theory (Montes et al., 2009; Ni, 1999), we opted for the two-film hypothesis.

The $\mathrm{NH}_{3}$ volatilisation rate $\left(\phi_{N_{H}}\right)$ is described in Eq. (2.11). The rate depends on the emitting area $\left(a_{p}\right)$, the convective mass transfer coefficient $\left(h_{m}\right)$, and the difference between $\mathrm{NH}_{3}-\mathrm{N}$ concentration in the puddle and in the air above. The $\mathrm{NH}_{3}-\mathrm{N}$ concentration in the air was fixed at $0 \mathrm{~mol} \mathrm{~m}^{-3}$ (Monteny et al., 1998). A factor $17 / 14$ was used to convert $\mathrm{NH}_{3}-\mathrm{N}$ to $\mathrm{NH}_{3}$ (Monteny et al., 1998).

$$
\phi_{N H_{3}}=a_{p} \cdot h_{m} \cdot 17 / 14 \cdot\left(\left[N H_{3}-N\right]_{\text {gas }, \text { bound }}-\left[N H_{3}-N\right]_{\text {gas }, \text { air }}\right)
$$

Empirical equations for $h_{m}$ vary substantially between studies. We identified the overall mass transfer coefficient in the gas phase $\left(K_{G A S}\right.$, Eq. (2.12)), and in the liquid phase $\left(K_{L I Q}\right.$, Eq. (2.13)) as described by Haslam et al. (1924), and Lewis \& Whitman (1924). This $K_{G A S}$ can be converted to $K_{L I Q}$ and vice versa with the Henry's law constant. Some authors only use the individual gas film mass transfer coefficient $\left(k_{\text {gas }}\right)$ (Haslam et al., 1924) as $h_{m}$ (Elzing \& Monteny, 1997). Others defined a different description and symbol (for example $K_{O L}$ ) that can be either $K_{G A S}$, or $K_{L I Q}$. We examined each equation in the literature and from its description in the paper determined whether it was equal to the individual $\left(k_{g a s}, k_{l i q}\right)$ or overall $\left(K_{G A S}, K_{L I Q}\right)$ mass transfer coefficient (Haslam et al., 1924). To compare equations, a standard unit was defined as $\mathrm{ms}^{-1}$. The complete list of equations is summarised in Table 2.4, in which each equation has been converted to this standardised unit.

We used $K_{G A S}$ to represent $h_{m}$ in each candidate model, according to Elzing $\&$ Monteny (1997). Figure 2.4 shows the dependence of $h_{m}$ on the air velocity $(v)$. We observed that for each equation, $h_{m}$ increased with increasing $v$. At higher $v$, differences of up to a factor 120 were observed between equations.

$$
\begin{gathered}
K_{G A S}=\frac{H k_{l i q} k_{g a s}}{H k_{l i q}+k_{g a s}}=\frac{1}{\frac{1}{k_{g a s}}+\frac{1}{k_{l i q}}} \\
K_{L I Q}=\frac{K_{G A S}}{H}=\frac{k_{l i q} k_{g a s}}{H k_{l i q}+k_{g a s}}=\frac{1}{\frac{H}{k_{g a s}}+\frac{1}{k_{l i q}}}
\end{gathered}
$$




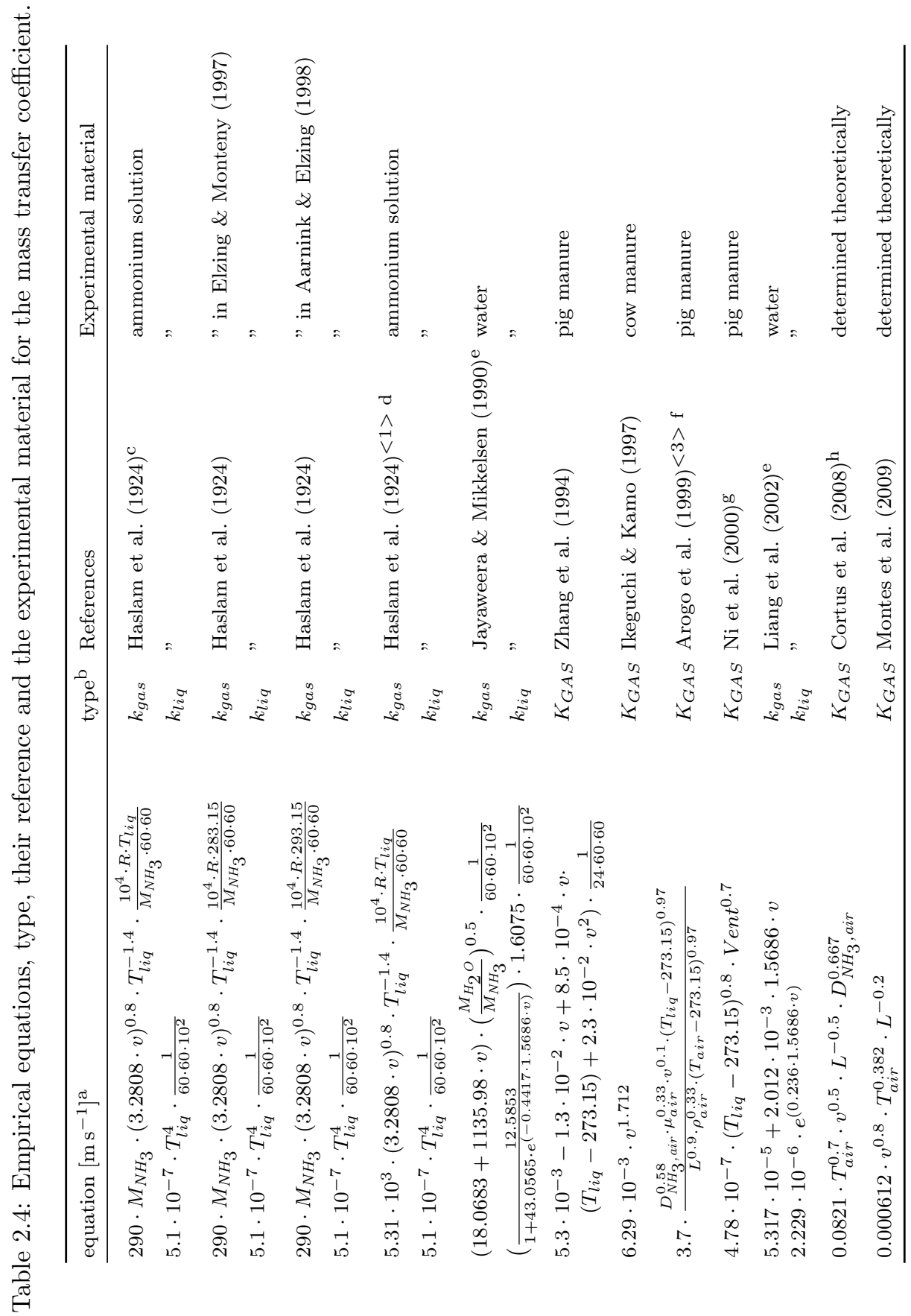




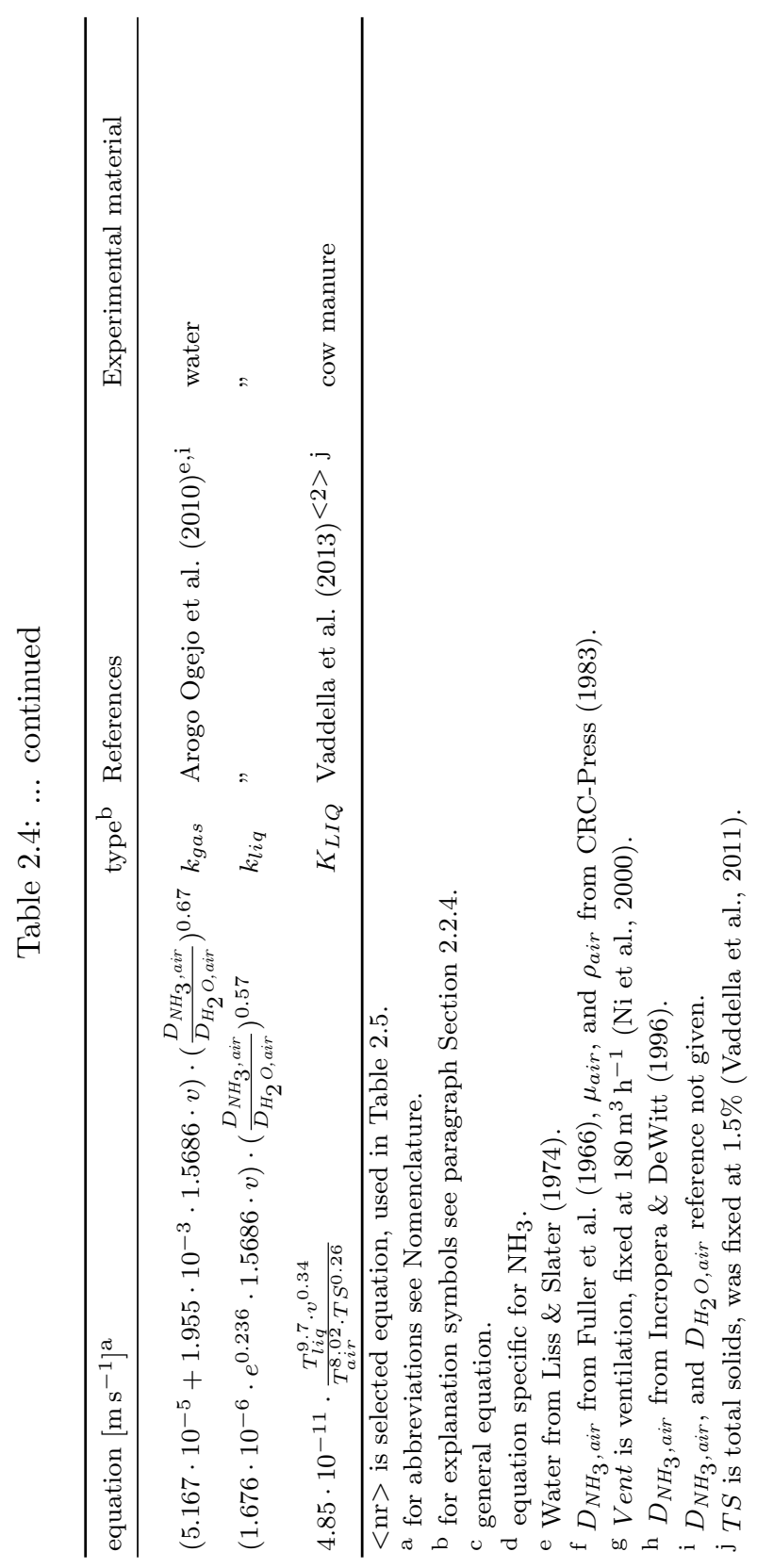




\subsubsection{The model}

Substitution of Eq. (2.10) into Eq. (2.11), and combining Eqs. (2.2) and (2.11) gives the complete mass balance for the $\mathrm{NH}_{3}$ emission process summarised in Eq. (2.14).

$$
\begin{aligned}
a_{p} \cdot d_{p} \cdot \frac{d[T A N]_{l i q}}{d t}=2 \cdot a_{p} \cdot d_{p} \cdot \frac{S m \cdot[U]_{l i q}}{K m+[U]_{l i q}}- \\
h_{m} \cdot a_{p} \cdot f \cdot\left([T A N]_{l i q}-\left[N H_{3}-N\right]_{g a s, a i r}\right) \cdot H_{i n v}^{c c}
\end{aligned}
$$

where $T A N$ is Total Ammoniacal Nitrogen, the sum of ammonia-nitrogen $\left(\mathrm{NH}_{3}-\mathrm{N}_{\text {liq }}\right)$ and ammonium-nitrogen $\left(\mathrm{NH}_{4}^{+}-\mathrm{N}_{\text {liq }}\right)$ (Monteny et al., 1998) and the number 2 follows from the urea conversion to $\mathrm{NH}_{3}$, Eq. (2.1).

At $t=\infty$ all urea converted to $\mathrm{NH}_{3}$, and emitted into the air. Equation (2.15) shows the theoretical maximum $\mathrm{NH}_{3}$ emission of a urine puddle $\left(\phi_{N_{H_{3}, \infty}}\right)$ :

$$
\phi_{N H_{3}, \infty}=\left[U_{0}\right]_{l i q} \cdot a_{p} \cdot d_{p} \cdot 17 / 14
$$

The three input variables $\left[U_{0}\right]_{l i q}, a_{p}$, and $d_{p}$ determine the $\mathrm{NH}_{3}$ source in the model. The other four input variables $\left(T_{a i r}, T_{l i q}, v\right.$, and $\left.p H\right)$, and the five model constants $\left(S m, K m, K_{a}, H_{i n v}^{c c}\right.$, and $\left.h_{m}\right)$ influence the volatilisation speed.

\subsubsection{Sensitivity analysis}

For each model parameter $K_{a}, H_{i n v}^{c c}$, and $h_{m}$, we selected three empirical equations, indicated in Tables 2.2 to 2.4. They were the highest, middle, or lowest equation in Figs. 2.2 to 2.4. We assumed that we could select each of the equations for our analysis because each was based on a liquid containing $\mathrm{NH}_{3}$. We used these three equations in each possible combination, thereby creating a $3 \cdot 3 \cdot 3=27$ candidate models of $\mathrm{NH}_{3}$ emission, as shown in Table 2.5.

For each candidate model we performed a variance-based Global Sensitivity Analysis as described in detail by Arogo Ogejo et al. (2010). This analysis determines the contribution of each input variable to the total variance of the output. For example $S_{x}$ of variable $x$, and similarly the interaction $S_{x, y}$ of variables $x$ and $y$ are calculated as follows:

$$
\begin{gathered}
S_{x}=\frac{v(E(y \mid x))}{V_{y}} \\
S_{x, y}=\frac{V(E(y \mid x, z))}{V_{y}}-\frac{V(E(y \mid x))}{V_{y}}-\frac{V(E(y \mid z))}{V_{y}}
\end{gathered}
$$

According to Arogo Ogejo et al. (2010) " $V_{y}$ is the variance of the model output, $y$, over $N$ simulations. The numerator is the variance of the expected value of $y$ given a 
constant $x$. For the analysis to be independent of the value of $x$, the above relationship was taken over all values of $x$ and averaged."

Similar to Arogo Ogejo et al. (2010), we determined the first-order $\left(S_{i}\right)$ and secondorder $\left(S_{i j}\right)$ sensitivity coefficient and the total effect index $\left(S_{T(i)}\right)$ for each input variable. The total effect index $S_{T(i)}$ was the summation of each first-, second-, and higher-order sensitivity coefficient related to one input variable. We determined $S_{T(i)}$ for the output $\phi_{N_{3}}\left(\mathrm{~mol} \mathrm{~s}^{-1}\right)$. To compare the candidate models, we introduced a sum-coefficient that contained the sum of each $S_{i}$ and $S_{i j}$ of the five most sensitive input variables.

Each input variable in Table 2.6 was a column in the matrices described by Arogo Ogejo et al. (2010). The number of simulations performed per matrix $(N)$ was set to 100000 . With nine input variables, this gave a resolution of $3.6\left(100000^{(1 / 9)}\right)$. The value for each input variable for each simulation was taken from a uniform distribution within the upper- and lower-limits (Table 2.6).

\subsubsection{Emission time interval}

We ran each simulation for emission durations of $5 \mathrm{~h}, 10.5 \mathrm{~h}$, and $20 \mathrm{~h}$. The emission duration $(e d)$ of $10.5 \mathrm{~h}$ was based on the average duration of undisturbed emission that elapsed before a urine puddle was flooded by a new one in the Monteny-model. This duration was determined according to Eq. (2.18), as described by Monteny et al. (1998).

$$
e d=\frac{d a y}{n c \cdot u f} \cdot \frac{n c \cdot a_{\text {floor }}}{a_{p}}
$$

where day is the number of hours in a day $(24 \mathrm{~h}), n c$ is the number of dairy cows in a cow house $(100 \#)$, uf is the urination frequency $\left(10 \mathrm{\#}^{-1} \mathrm{cow}^{-1}\right), a_{\text {floor }}$ is the total floor area per dairy cow $\left(3.5 \mathrm{~m}^{2} \mathrm{cow}^{-1}\right)$, and $a_{p}$ is the area covered by a urine puddle $\left(0.8 \mathrm{~m}^{2}\right)$. These values were based on the reference cow house in the currently used model (Monteny et al., 1998). The durations of $5 \mathrm{~h}$, and $20 \mathrm{~h}$ allowed us to assess the influence of emission duration in relation to the reference duration of $10.5 \mathrm{~h}$. 
Table 2.5: Sensitivity analysis approach part one: define candidate models on basis of selected equations for the model parameters $K_{a}, H_{i n v}^{c c}$, and $h_{m}$.

\begin{tabular}{cccc}
\hline \multirow{2}{*}{ candidate model } & \multicolumn{3}{c}{ constant } \\
\cline { 2 - 4 } & $K_{a}$ & $H_{\text {inv }}^{c c}$ & $h_{m}$ \\
\hline 1 & 1 & 1 & 1 \\
2 & 1 & 1 & 2 \\
3 & 1 & 1 & 3 \\
4 & 1 & 2 & 1 \\
5 & 1 & 2 & 2 \\
$\vdots$ & $\vdots$ & $\vdots$ & $\vdots$ \\
27 & 3 & 3 & 3 \\
\hline
\end{tabular}

Table 2.6: Sensitivity analysis approach part two: input variables (var) and their extreme low and high values on basis of data from literature.

\begin{tabular}{llll}
\hline Var & Low & High & References \\
\hline$\left[U_{0}\right]_{l i q}$ & 85.7 & 432.0 & Monteny et al. (2002); van Duinkerken et al. (2003) \\
$T_{\text {air }}$ & 273.15 & 308.15 & Scholtens \& Huis in 't Veld (1997) \\
$T_{l i q}$ & 273.15 & 311.15 & van Duinkerken (2012) \\
$v$ & 0.05 & 0.55 & Schrade et al. (2012) \\
$a_{p}$ & 0.4 & 1.8 & Braam \& van den Hoorn (1996) \\
$d_{p}$ & $1.3 \times 10^{-4}$ & $1.6 \times 10^{-3}$ & " \\
$p H$ & 6.9 & 9.7 & Monteny et al. (2002); DeGroot et al. $(2010)$ \\
$S_{m}$ & $7.9 \times 10^{-4}$ & $5.8 \times 10^{-1}$ & see Table 2.1 \\
$K_{m}$ & 1.8 & 2.2 & $\pm 10 \%$ of reference value \\
\hline
\end{tabular}




\section{$2.3 \quad$ Results}

The first- and second-order sensitivity coefficients $\left(S_{i}\right.$ and $\left.S_{i j}\right)$ are listed in Table 2.7. The sum of all $S_{i}$ was 0.63 for emission duration $1\left(e d_{1}\right), 0.66$ for $e d_{2}$, and 0.69 for $e d_{3}$. For $e d_{1}$, and $e d_{2} S_{p H}$ was largest. The $S_{d_{p}}$ was largest for $e d_{3}$, and this coefficient increased when $(e d)$ increased (Table 2.7). The same trend can be observed in the sensitivity of variables $\left[U_{0}\right]_{l i q}$, and $a_{p}$. These three variables together describe the $\mathrm{NH}_{3}$ source (Section 2.2.5). The coefficients $S_{p H}$, and $S_{T_{l i q}}$ decreased when ed increased. The remaining four coefficients were $\leq 0.01$.

The sum of all $S_{i j}$ was 0.32 for $e d_{1}, 0.30$ for $e d_{2}$, and 0.28 for $e d_{3}$ (Table 2.7). The coefficient $S_{d_{p} \cdot p H}$ was largest for each $e d$, but was never greater than 0.05. Each coefficient for only $\mathrm{NH}_{3}$ source-related variables showed an increased $S_{i}$ when ed increased. Combinations with $p H$, or $T_{l i q}$ showed a decreased $S_{i}$ when $e d$ increased.

Figure 2.5 shows the sum of each $S_{i}$ and $S_{i j}$ for $p H, d_{p},\left[U_{0}\right]_{l i q}, a_{p}$, and $T_{l i q}$, called the sum-coefficients. The sum-coefficient for each candidate model and also for each $e d$ was between 0.71 and 0.97 . The lowest sum-coefficients were for candidate models $3,6,9,12,15,18,21,24$ and 27. All these candidate models contained $h_{m}$ nr 3 (Table 2.5), which was the bottom line in Fig. 2.4: see Table 2.4 (Arogo et al., 1999). The Global Sensitivity Analysis results revealed a trend. The coefficient $S_{i}$ for each $\mathrm{NH}_{3}$ source-related input variable $\left(i=d_{p},\left[U_{0}\right]_{l i q}, a_{p}\right)$ was smaller for candidate models with $h_{m}$ nr 3 than for each other model. Variables $T_{l i q}$, and $T_{a i r}$ showed the opposite trend: the coefficient $S_{T_{l i q}}$ was larger for candidate models with $h_{m}$ nr 3 than for each other model. The coefficient $S_{T_{a i r}}$ had a value for candidate models with $h_{m}$ nr 3 , but was zero for each other model. The sensitivity coefficient for variable $p H$ did not show this trend.

The total effect index $\left(S_{T(i)}\right)$ showed that $S_{T(p H)}$ was largest for runs $e d_{1}$, and $e d_{2}$, followed by $S_{T\left(d_{p}\right)}$ (Table 2.8). For run $e d_{3}$ the models were also most sensitive to these two variables, but in the reverse order. For run $e d_{2}$ and $e d_{3}$, the sensitivity of models to variable $\left[U_{0}\right]_{l i q}$ came third, followed by variable $a_{p}$ and variable $T_{l i q}$. For run $e d_{1}$ the variable to which the model was third most sensitive was $T_{l i q}$, followed by $\left[U_{0}\right]_{l i q}$, and $a_{p}$. For each $e d$ the lowest sensitivity was to input variables $T_{a i r}, v, S m$, and $K m$, for which the coefficients were $\leq 0.05$. 
Table 2.7: First- (upper part) and second-order (lower part) model sensitivity coefficients for input variables (var), given as mean (with SD) of all 27 candidate models for emission durations (ed) $5 \mathrm{~h}, 10.5 \mathrm{~h}$, and $20 \mathrm{~h}$.

\begin{tabular}{lccc}
\hline \multirow{2}{*}{ Var } & \multicolumn{3}{c}{ Sensitivity coefficient, ${ }^{\mathrm{a}}$ mean $(\mathrm{SD})$} \\
\cline { 2 - 4 } & $e d_{1}=5 \mathrm{~h}$ & $e d_{2}=10.5 \mathrm{~h}$ & $e d_{3}=20 \mathrm{~h}$ \\
\hline First-order coefficients $\left(S_{i}\right)$ & $0.17(0.068)$ & $0.13(0.071)$ \\
$p H$ & $0.21(0.064)$ & $0.15(0.091)$ & $0.19(0.101)$ \\
$d_{p}$ & $0.12(0.076)$ & $0.15(0.059)$ \\
{$\left[U_{0}\right]_{l i q}$} & $0.10(0.050)$ & $0.13(0.057)$ & $0.15(0.059)$ \\
$a_{p}$ & $0.09(0.046)$ & $0.12(0.052)$ & $0.13(0.054)$ \\
$T_{l i q}$ & $0.09(0.067)$ & $0.08(0.072)$ & $0.07(0.073)$ \\
$T_{a i r}$ & $0.01(0.020)$ & $0.01(0.016)$ & $0.01(0.013)$ \\
$v$ & $0.00(0.004)$ & $0.00(0.003)$ & $0.00(0.002)$ \\
$S_{m}$ & $0.00(0.001)$ & $0.00(0.000)$ & $0.00(0.000)$ \\
$K_{m}$ & $0.00(0.000)$ & $0.00(0.000)$ & $0.00(0.000)$ \\
\hline Total & $0.63(0.072)$ & $0.66(0.072)$ & $0.69(0.072)$
\end{tabular}

Second-order coefficients $\left(S_{i j}\right)^{\mathrm{b}}$

\begin{tabular}{lccc}
$d_{p} \cdot p H$ & $0.05(0.021)$ & $0.05(0.017)$ & $0.04(0.015)$ \\
$T_{l i q} \cdot p H$ & $0.05(0.034)$ & $0.04(0.023)$ & $0.03(0.016)$ \\
$a_{p} \cdot d_{p}$ & $0.02(0.012)$ & $0.03(0.013)$ & $0.03(0.014)$ \\
{$\left[U_{0}\right]_{l i q} \cdot p H$} & $0.03(0.009)$ & $0.03(0.009)$ & $0.02(0.010)$ \\
$a_{p} \cdot p H$ & $0.03(0.009)$ & $0.02(0.009)$ & $0.02(0.010)$ \\
{$\left[U_{0}\right]_{l i q} \cdot d_{p}$} & $0.02(0.011)$ & $0.02(0.013)$ & $0.03(0.014)$ \\
{$\left[U_{0}\right]_{l i q} \cdot a_{p}$} & $0.02(0.007)$ & $0.02(0.007)$ & $0.02(0.007)$ \\
$T_{l i q} \cdot d_{p}$ & $0.02(0.008)$ & $0.02(0.012)$ & $0.02(0.014)$ \\
{$\left[U_{0}\right]_{l i q} \cdot T_{l i q}$} & $0.02(0.012)$ & $0.02(0.012)$ & $0.02(0.012)$ \\
$T_{l i q} \cdot a_{p}$ & $0.01(0.009)$ & $0.01(0.010)$ & $0.01(0.010)$ \\
$T_{a i r} \cdot p H$ & $0.01(0.009)$ & - & - \\
$T_{a i r} \cdot T_{l i q}$ & $0.01(0.007)$ & - & - \\
$T_{a i r} \cdot d_{p}$ & $0.01(0.005)$ & - & - \\
\hline Total & $0.32(0.035)$ & $0.30(0.040)$ & $0.28(0.044)$
\end{tabular}

${ }^{a}$ Decreasing order in column ed $2(10.5 \mathrm{~h})$.

b A total of 36 second order effects were calculated. Only those with an effect $\geq 0.01$ are reported. 
Table 2.8: Total effect index of each input variable, given as mean (with SD) of all 27 candidate models for emission durations (ed) $5 \mathrm{~h}, 10.5 \mathrm{~h}$, and $20 \mathrm{~h}$.

\begin{tabular}{lccc}
\hline \multirow{2}{*}{$\operatorname{var}$} & \multicolumn{3}{c}{ Total effect index, mean (SD) } \\
\cline { 2 - 4 } & $e d_{1}=5 \mathrm{~h}$ & $e d_{2}=10.5 \mathrm{~h}$ & $e d_{3}=20 \mathrm{~h}$ \\
\hline$p H$ & $0.43(0.106)$ & $0.36(0.123)$ & $0.29(0.132)$ \\
$d_{p}$ & $0.27(0.089)$ & $0.32(0.093)$ & $0.35(0.094)$ \\
{$\left[U_{0}\right]_{l i q}$} & $0.22(0.045)$ & $0.25(0.049)$ & $0.26(0.051)$ \\
$a_{p}$ & $0.21(0.039)$ & $0.23(0.044)$ & $0.24(0.047)$ \\
$T_{l i q}$ & $0.24(0.165)$ & $0.22(0.159)$ & $0.19(0.151)$ \\
$T_{a i r}$ & $0.05(0.070)$ & $0.04(0.052)$ & $0.03(0.041)$ \\
$v$ & $0.01(0.014)$ & $0.01(0.011)$ & $0.01(0.009)$ \\
$S_{m}$ & $0.01(0.000)$ & $0.00(0.000)$ & $0.00(0.000)$ \\
$K_{m}$ & $0.00(0.000)$ & $0.00(0.000)$ & $0.00(0.000)$ \\
\hline
\end{tabular}

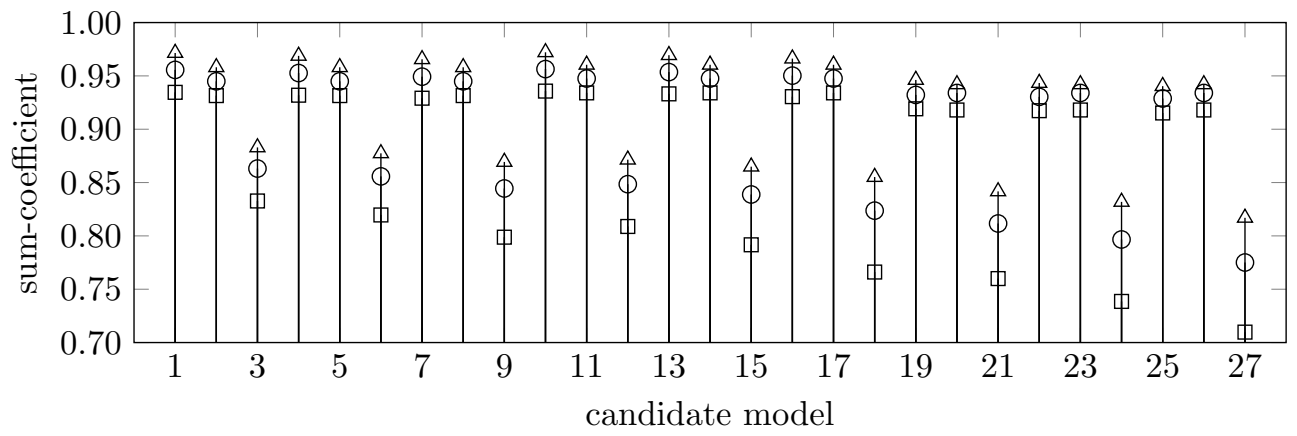

Figure 2.5: Summed first- and second-order sensitivity coefficients of $p H, d_{p},\left[U_{0}\right]_{l i q}$, $a_{p}$, and $T_{l i q}$, for 27 candidate models with varying model constants $K_{a}, H_{i n v}^{c c}$, and $h_{m}$ (Table 2.5), for $e d_{1} \square$, $e d_{2} \circ$, and $e d_{3} \Delta$. 


\subsection{Discussion}

The emission duration of one urine puddle $(e d)$, and the selected empirical equations for the model parameters influenced the ranking of sensitivity to input variables in the Global Sensitivity Analysis method applied. But in each run (5h, $10.5 \mathrm{~h}$ and $20 \mathrm{~h}$ ), the top five greatest sensitivity coefficients were for input variables $p H, d_{p},\left[U_{0}\right]_{l i q}, a_{p}$ and $T_{l i q}$. For each of these variables the total effect index was at least 0.19 compared to $\leq 0.05$ for $T_{l i q}, v, S_{m}$, and $K_{m}$. In each candidate model, the sum of all first-order $\left(S_{i}\right)$ and second-order sensitivity coefficient $\left(S_{i j}\right)$ (sum-coefficient) of these top five sensitivity coefficients was between 0.71 and 0.97 .

The lowest sum-coefficient values, 0.71 to 0.88 , occurred when a candidate model contained $h_{m} \mathrm{nr} 3$. This $h_{m}$ represented a slow mass transfer of ammonia $\left(\mathrm{NH}_{3}\right)$ from puddle into air (Figure 2.4), resulting in a low $\mathrm{NH}_{3}$ emission. Results showed that in these candidate models the $S_{i}$ for the variables related to speed of emission $(i=p H$, $\left.T_{l i q}, T_{l i q}\right)$ were high, whereas the variables related to $\mathrm{NH}_{3}$ source $\left(i=d_{p},\left[U_{0}\right]_{l i q}, a_{p}\right)$ were low. The $S_{i}$ varied for each input variable within each other candidate model, but overall the sum-coefficient was at least 0.92 . This means that at least $92 \%$ of the variation can be explained.

The empirical equations used for the model parameters (i. e.the dissociation constant, Henry's law constant and the mass transfer coefficient) were based on ammonium solutions and some on manure from various types of livestock. We assumed that each equation could be used in the $\mathrm{NH}_{3}$ emission model for urine puddles because basically each equation described a liquid containing $\mathrm{NH}_{3}$, which is the case for a urine puddle.

The models were most sensitive to input variable $p H$ in runs $5 \mathrm{~h}$ and $10.5 \mathrm{~h}$, and the second most sensitive in run $20 \mathrm{~h}$. This high sensitivity can be explained. The $p H$ determined the equilibrium between $\mathrm{NH}_{3}$, and $\mathrm{NH}_{4}^{+}$, together with the dissociation constant. This equilibrium is an S-shaped curve. For $p H$ values below approximately 8 there is only $\mathrm{NH}_{4}^{+}$present in a puddle, and above approximately 11.5 there is only $\mathrm{NH}_{3}$. In between, the $\mathrm{NH}_{3}$ concentration increases linearly. The selected $p H$ values in this study cover almost the complete $\mathrm{S}$-curve, resulting in low and high $\mathrm{NH}_{3}$ emission rates.

The second, third and fourth greatest sensitivities were to input variables $d_{p}$, $\left[U_{0}\right]_{l i q}, a_{p}$ respectively. They determined the source size and thus the potential total amount of $\mathrm{NH}_{3}$ available for emission. Higher values resulted in more $\mathrm{NH}_{3}$ and higher emission rates. Additionally, when $e d$ increased these source-related variables became more important, whereas the other, speed-related, variables became less important. Thus, if duration is less restrictive, source size will become the limiting factor and thus more important.

In this study the models were least sensitive to input variables $T_{a i r}$ and $v$. Aarnink \& Elzing (1998) also showed minor effects for $T$ and $v$ on floor level. In their study 
the $T$ and $v$ effects related to the slurry pit were much greater. In agreement with Monteny et al. (1998), $T_{a i r}$ and $v_{\text {air }}$ were among the variables to have a marked effect but, similar to Aarnink \& Elzing (1998), the effects related to the slurry pit were greater. Both Aarnink \& Elzing (1998) and Monteny et al. (1998) analysed the sensitivity of $\mathrm{NH}_{3}$ emission in an animal house, considering the total $\mathrm{NH}_{3}$ emission from urine puddles plus the slurry pit. Therefore the effects for an individual urine puddle cannot be derived from their studies.

Similar to Monteny et al. (1998), we assumed that $\mathrm{NH}_{3}$ concentration in the air above the urine puddle was $0 \mathrm{~mol} \mathrm{~m}^{-3}$. We performed an additional run with a concentration of $4.3 \times 10^{-4} \mathrm{~mol} \mathrm{~m}^{-3}(10 \mathrm{ppm})$ for $e d_{2}$. The results of this run were similar to those for $e d_{2}$ in Tables 2.7, and 2.8. A concentration of $10 \mathrm{ppm}$ represented the approximate average upper range of $\mathrm{NH}_{3}$ concentration in a Dutch dairy cow house, and was assumed to apply to the air throughout the house. In each simulation there was no concentration gradient in the air just above a puddle, and dynamic modelling was not included.

The results showed that input variable $S_{m}$ did not affect $\mathrm{NH}_{3}$ emission. However, this $S_{m}$ did not show a linear relation with $\mathrm{NH}_{3}$ emission (Monteny et al., 1998) and thus may increase the estimated sensitivity coefficient. Monteny showed that for extremely low $S_{m}$ values the $\mathrm{NH}_{3}$ emission was low, but that this emission increased rapidly when $S_{m}$ increased slightly. Low $S_{m}$ values do not occur in commercial dairy cow houses in the Netherlands that have a concrete floor. However, this could change with the introduction of new types of floor, the use of urease inhibitors, or other methods in future. This example demonstrates that the specific response of a parameter may lead to a low sensitivity coefficient and underestimation of its relevance in specific input variable settings. Nevertheless we expect that in our study such behaviour is restricted to the parameter $S_{m}$.

The $\mathrm{NH}_{3}$ emission model uses $p H$ as input variable. However, $p H$ is the result not only of the $\mathrm{NH}_{3}$ in the urine but also of other components of urine, chemical processes in urine and the emission of other gases. We did not find a model that used urine composition data as input to predict the $\mathrm{pH}$ of a liquid.

Note that random combinations of variables within the set bandwidths (Table 2.6) may in some cases lead to unrealistic values that will never apply in practice. To avoid this we would have to include distributions, relationships and expected correlations between input variables. However, no reliable information was available on such distributions and relationships. Besides, our research focussed on the $\mathrm{NH}_{3}$ emission from only one urine puddle per simulation. In practice, individual puddles may be extreme for one or more variables that lead to extreme emissions. Therefore we have no reason to expect that the observed ranking in sensitivity coefficients in Tables 2.7 and 2.8 will be affected. 


\subsection{Conclusions}

Our objective was to improve the $\mathrm{NH}_{3}$ emission model by gaining insight into the sensitivity to model parameters and input variables involved in the emission from urine puddles as a first step.

We found that the $\mathrm{NH}_{3}$ emission of one urine puddle on a floor was sensitive for input variables $p H, d_{p},\left[U_{0}\right]_{l i q}, a_{p}$, and $T_{l i q}$. The sum of each first- and second-order sensitivity coefficient of these variables (sum-coefficient) ranged from 0.71 to 0.97 . This means that $71 \%$ to $97 \%$ of the variation in $\mathrm{NH}_{3}$ emission can be explained by five input variables. These values hold for each candidate model; each such model contained varying model parameters and both had low and high input values. The four remaining variables $T_{a i r}, v, S_{m}$, and $K_{m}$ did not contribute substantially to the variation in the output.

The sum-coefficient of these five sensitive variables to which the candidate models were most sensitive ranged from 0.71 to 0.88 when the model contained the version of the mass transfer coefficient that represents the lowest values. In each other candidate model the value for this sum-coefficient was at least 0.92 .

Based on our conclusions we recommend simplifying the model structurally and reducing the number of input variables. The variation in $\mathrm{NH}_{3}$ emission of individual urine puddles can be explained by five input variables. These variables need to be measured in practice to validate the model.

\subsection{Acknowledgements}

The authors acknowledge the assistance of S. Blaauw during the simulation work. Language editing of a near-final draft of the paper was provided by J. Burrough. 
CHAPTER 3

\section{Dynamic behaviour of $\mathrm{pH}$ in fresh urine puddles of dairy cows}

Dennis Snoek

Nico Ogink

Hans Stigter

Sido Agricola

Teun van de Weijer

Peter Groot Koerkamp

Published in Transactions of the ASABE (2016), 59(5)

Reproduced with permission of ASABE $\left.{ }^{(}\right)$ 


\begin{abstract}
Modern livestock farming is an important contributor to ammonia $\left(\mathrm{NH}_{3}\right)$ emission. In the Netherlands, $94 \%$ of $\mathrm{NH}_{3}$ emissions originate from agriculture, of which $34 \%$ is emitted from commercial dairy cow houses. From current mechanistic modelling it is known that the $p H$ of urine puddles from cows is one of the most important variables to estimate $\mathrm{NH}_{3}$ emission. However, little $p H$ data are available from commercial cow houses. Therefore, the objective of this study was to investigate $p H$ values and to study their dynamic behaviour in fresh, on-floor urine puddles in these houses. To do this, the $p H$ of urine puddles was measured for $4 \mathrm{~h}$ per puddle, and a model was developed to describe the $p H$ behaviour. In total, 26 fresh puddles were measured from cows at three commercial dairy farms in summer and winter. At farm level, we found initial $p H$ values of 8.1 through 8.4, which increased to 8.9 through 9.4 after $4 \mathrm{~h}$. The $p H$ difference between summer and winter was $0.3(\mathrm{p}<0.05)$, but this was not confirmed by comparisons at farm level. The $p H$ curves of individual puddles varied substantially and could be fitted by a nonlinear regression model. This model contained correlated coefficients that were able to describe the main, known chemical processes of a urine puddle. However, no linear relation was found between initial and final $p H$ and thus between coefficients. On average, $p H$ quickly increased initially, declined after $1 \mathrm{~h}$ and became stable around a $p H$ of 9.15 . We conclude that a $p H$ curve will better describe the input variable in $\mathrm{NH}_{3}$ emission modelling than the current situation of using a static $p H$ value. Based on this study, we recommend to use the mean measured $p H$ curve as input for the puddle simulation during $\mathrm{NH}_{3}$ emission modelling of dairy cow houses.
\end{abstract}




\subsection{Introduction}

The deposition of $\mathrm{NH}_{3}$ contributes to the acidification and eutrophication of the environment. In the Netherlands, $94 \%$ of the $\mathrm{NH}_{3}$ emissions originate from agriculture (EEA, 2012), of which $34 \%$ is emitted from dairy cow houses and their manure storage facilities. In a typical Dutch dairy cow house, approximately $70 \%$ of the emitted $\mathrm{NH}_{3}$ comes from urine on a slatted floor (Monteny et al., 1998), while the remaining $\mathrm{NH}_{3}$ is emitted from the manure stored below the floor. Even though in 2010 the Dutch $\mathrm{NH}_{3}$ emission was just below the National Emission Ceilings (NEC) for the EU member states (EU, 2001; UNECE, 1999; EEA, 2012), the Dutch National Institute for Public Health and the Environment (RIVM) expects that $\mathrm{NH}_{3}$ emissions in the Netherlands may soon exceed the NEC threshold. Indeed, local and regional $\mathrm{NH}_{3}$ emissions have already caused overloads in nature reserves like the Natura2000 areas (PBL, 2012). This growing threat along with an expected lower NEC in the future and stricter EU emission regulations call for the development of dairy cow houses with a lower $\mathrm{NH}_{3}$ emission than that of current houses. However, before these houses can be designed, the dynamic behaviour of $\mathrm{NH}_{3}$ from the current houses needs to be understood. With a better understanding, point of improvement can be identified and implemented.

Several mechanistic models have been developed to study $\mathrm{NH}_{3}$ emission-related processes and to estimate $\mathrm{NH}_{3}$ emission of dairy cow houses (Elzing \& Monteny, 1997; Monteny et al., 1998; Montes et al., 2009; Vaddella et al., 2013; Hafner et al., 2013). However, according to Snoek et al. (2014b) the empirical equations for the model parameters vary among these models. In practise many of the input variables cannot be measured or assessed accurately and their actual influence on the $\mathrm{NH}_{3}$ emission is thus unknown. In their study Snoek et al. (2014b) performed a sensitivity analysis of these models and demonstrated that the variation in $\mathrm{NH}_{3}$ emission can be explained for at least $71 \%$ by five puddle-related input variables: $1 . \mathrm{pH}, 2$. depth, 3. urea concentration, 4. area, and 5. temperature. Furthermore, this study found that these models seldom used input from commercial dairy houses. This is most likely due to both a lack of proper measurement methods and the difficulty of measuring this data.

In the current mechanistic models, the input variable $p H$ is assumed to be a constant value with a pronounced effect on estimated $\mathrm{NH}_{3}$ emission (Aarnink \& Elzing, 1998; Chaoui et al., 2009; Elzing \& Monteny, 1997; Monteny et al., 1998). This assumption is questionable, because $\mathrm{NH}_{3}$ emission is a dynamic process in which the $p H$ is an indicator and not an input variable (Blanes-Vidal et al., 2009; Chaoui et al., 2009; Monteny, 2000; Sommer \& Sherlock, 1996). According to Blanes-Vidal et al. (2009), Chaoui et al. (2009) and Hafner et al. (2013), the $p H$ initially increases because acid gas $\mathrm{CO}_{2}$ is emitted. This emission is initially faster than the emission of the base gas $\mathrm{NH}_{3}$. After this increase, the rate of $p H$ change slows since the $p H$ increase favours the emission of basic components and hinders the emission of acidic components. In 
Table 3.1: Experimental design with the farms, season $(\mathrm{S}=$ summer, $\mathrm{W}=$ winter $)$, the related code, and the number of measured fresh dairy cow urine puddles.

\begin{tabular}{cccc}
\hline Farm & Season & Code & Puddles \\
\hline 1 & S & S1 & 7 \\
2 & S & S2 & 5 \\
3 & - & - & - \\
1 & W & W1 & 7 \\
2 & - & - & - \\
3 & W & W3 & 7 \\
\hline
\end{tabular}

their study, Blanes-Vidal et al. (2009) used the $p H$ curve of the surface layer of a pig waste lagoon (Blunden \& Aneja, 2008). This $p H$ curve is similar to the measured $p H$ curves for cow urine and slurry (Monteny, 2000). Monteny (2000) showed that the dynamic $p H$ curve was explained by a non-linear regression model consisting of an exponential curve with a linear asymptote. He performed lab measurements on tiles and slurry, and performed an indicative cow house measurement. The slurry and house measurements showed higher $p H$ values than the tile measurements. Therefore, he hypothesised that $p H$ values measured inside cow houses may be higher compared to lab experiments. Finally, the mentioned studies (Blunden \& Aneja, 2008; Chaoui et al., 2009; Monteny, 2000) were performed with closed jars in a lab. Thus, they did not represent the actual situation in a cow house.

To the best of our knowledge, $p H$ values or time series of fresh urine puddles from commercial dairy cow houses have not yet been reported. The objective of this study was to investigate $p H$ values and to study their dynamic behaviour in fresh on-floor urine puddles in commercial dairy cow houses. To this end, we performed $p H$ measurements for $4 \mathrm{~h}$ per puddle, and a mechanistic model was introduced to describe these measured $p H$ values. For future modelling purposes, we also measured and reported puddle temperature, local air temperature, and local air humidity.

\subsection{Materials and Methods}

Sections 3.2.1 to 3.2.3 describe the experimental design, the equipment used and the calibrations. Section 3.2.4 describes the analyses, which were performed Matlab ${ }^{\circledR}$ R2015b (Mathworks, USA).

\subsubsection{Experimental design}

To investigate $p H$ and its behaviour, we measured 26 fresh urine puddles from dairy cows. Puddles were sampled at three farms and measured in two seasons. Two farms 
(1 and 2) were measured in summer (July 2014), and two farms (1 and 3) in winter (March 2015) (Table 3.1). We measured the $p H$ and temperature $\left(T_{l i q}\right)$ of each puddle, and air temperature $\left(T_{a i r}\right)$ and relative humidity $(R H)$ just above the puddle. Each measurement was started as soon as the cow had finished urinating and was taken for $15000 \mathrm{~s}(>4 \mathrm{~h})$ per puddle with a $15 \mathrm{~s}$ interval.

Each farm was located near Wageningen, The Netherlands, and each dairy cow house had a slatted concrete floor. In the summer, cows grazed during the day and received grass silage and maize at night. In the winter, cows were fed twice a day with grass silage $(1 / 3)$ and maize $(2 / 3)$. The cows also received basic concentrates and unlimited access to water in both summer and winter.

Farms 1 and 2 did not use a manure scraper, whereas farm 3 had a manure scraper robot. Farm 3 used an automatic milking system (AMS), whereas farms 1 and 2 used a conventional milking parlour and milked twice a day. Because farm 1 and 2 pastured the cows in summer, the measurements in summer were performed just after the milking in the morning. Farm 1 started milking early in the morning, whereas farm 2 started late in the morning or even in the beginning of the afternoon. Therefore, farm 1 was always measured first, followed by farm 2 . In the winter, we alternated the order of a morning and an afternoon measurement among farms 1 and 3. During each measurement, we fenced off the measurement area to protect the equipment and the urine puddle.

\subsubsection{Equipment}

Figure 3.1 shows the measurement equipment attached to a tripod and Table 3.2 gives the equipment's details. Each puddle was measured with a $p H$ sensor connected to a portable $p H / O R P$ meter (Horiba Ltd, JPN). The measurement equipment also measured $T_{l i q}$ to give correct $p H$ values. However, from preliminary tests we concluded that this temperature measurement was too slow for our purpose and therefore not accurate enough. Because of this, $T_{l i q}$ was also measured with a fast-responding temperature sensor connected to a multifunction meter (Testo AG, GER). The $T_{l i q}$ sensor was encased in a protective tube and then attached to the $p H$ sensor. Another sensor was connected to measure $R H$ and $T_{a i r}$ of the air. This sensor was located about $1 \mathrm{~m}$ above the puddle.

The $p H$ sensor automatically switched off after about one hour to save battery power. Because of this the sensor had to be switched on again each hour manually. Occasionally it happened that the sensor was switched off during the collection of some individual data points by the $p H$ logger. This resulted in some missing values. In addition, not each curve was exactly $15000 \mathrm{~s}$, but some were slightly shorter. 
Table 3.2: Sensors used for each variable (Var), their accuracy and related range.

\begin{tabular}{llll}
\hline Var & Sensor & Accuracy & (range) \\
\hline$p H$ & Horiba ISFET 0040-10D electrode, sensor model 0141 & $\pm 0.01 \mathrm{pH}$ & $(0$ to $14 \mathrm{pH})$ \\
$T_{l i q}$ & Horiba ISFET 0040-10D electrode, sensor model 0141 & $\pm 1{ }^{\circ} \mathrm{C}$ & $\left(0\right.$ to $\left.100{ }^{\circ} \mathrm{C}\right)$ \\
$T_{l i q}$ & Testo WP fast-action immersion probe: 0602 0493 & $\pm 0.09{ }^{\circ} \mathrm{C}$ & $\left(0\right.$ to $\left.30{ }^{\circ} \mathrm{C}\right)$ \\
$R H$ & Testo $R H / T_{a i r}$ probe: 06369735 & $\pm 2 \%$ & $(0$ to $100 \%)$ \\
$T_{a i r}$ & Testo $R H / T_{a i r}$ probe: 06369735 & $\pm 0.3^{\circ} \mathrm{C}$ & $\left(-20\right.$ to $\left.70{ }^{\circ} \mathrm{C}\right)$ \\
\hline
\end{tabular}
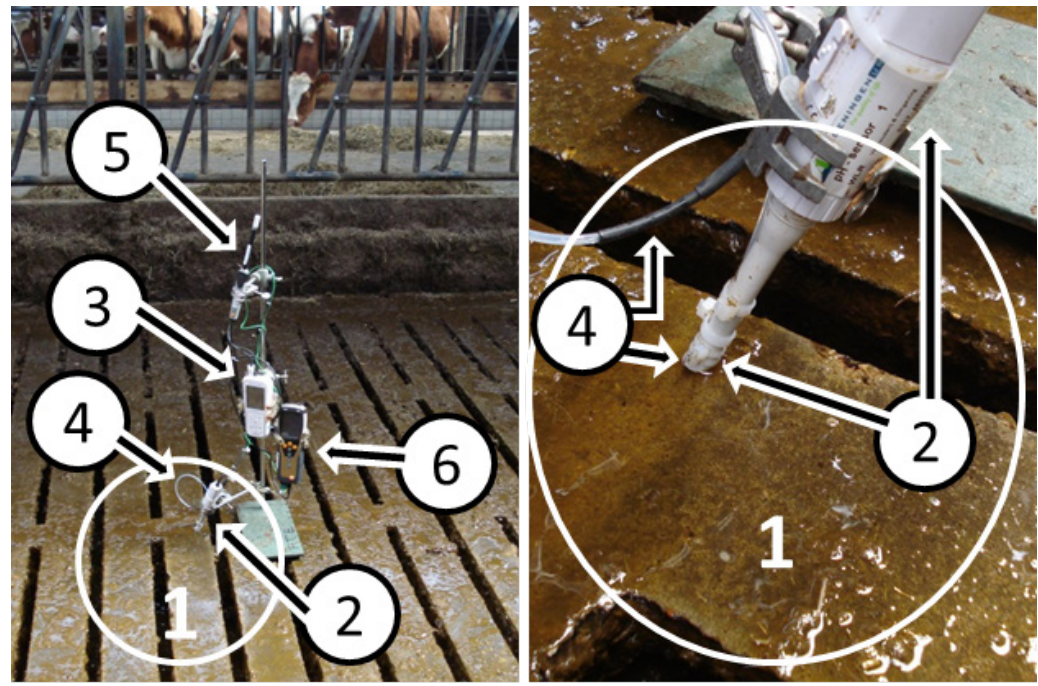

Figure 3.1: A fresh dairy cow urine puddle (1), and the tripod with the $p H$ sensor (2), $p H$ logger (3), $T_{l i q}$ sensor (4), $T_{a i r} / R H$ sensor (5), and $T_{l i q} / T_{a i r} / R H$ logger (6). Details of the sensors used are given in Table 3.2.

\subsubsection{Calibration}

Before each puddle was measured, a two-point calibration of the $p H$ sensor was performed on-site. This was done with two calibration buffer solutions: HI70007P with a $p H$ of $7.01 \pm 0.01$ and HI70010P with a $p H$ of $10.01 \pm 0.01$ and both at $25^{\circ} \mathrm{C}$ (Hanna Instruments ${ }^{\circledR}$, USA). The Testo sensors were calibrated in the lab using a F250 Precision Thermometer $\left( \pm 0.005^{\circ} \mathrm{C}\right.$, ASL, USA $)$.

The $p H$ sensor automatically corrected the measured $p H$ for temperature according to the Nernst equation. The $p H$ was re-corrected afterwards based on the $T_{l i q}$ measured by the fast-responding temperature sensor (Section 3.2.2).

In a preliminary experiment we did trials with measurements in thin fluid layers of 
buffer solutions with known $p H$ value. From these tests was concluded that the used $\mathrm{pH}$ sensor was able to measure these thin layers with an accuracy of $<0.1 \mathrm{pH}$.

\subsubsection{Statistical analyses}

\section{$\mathrm{pH}$ values}

For each measured puddle, the initial $p H$ value and the value at each hour were selected from the data, i.e., at $t=0 \mathrm{~s}\left(p H_{t_{0}}\right), t=3600 \mathrm{~s}\left(p H_{t_{1}}\right), t=7200 \mathrm{~s}\left(p H_{t_{2}}\right), t=$ $10800 \mathrm{~s}\left(p H_{t_{3}}\right)$, and $t=14400 \mathrm{~s}\left(p H_{t_{4}}\right)$. The highest $p H$ value in a curve was defined as $p H_{\max }$. For each of the selected $p H_{t}$ values, the mean and standard deviation were determined per farm, per season and overall. The assumption of normality was confirmed with the Shapiro-Wilk test, and the assumption of homogeneity of variances was confirmed with the Levene's test. A one-way ANOVA with the Tukey HSD post-hoc test was performed to compare the means of each farm. In addition, a two-sided independent samples Student's $t$-test was performed to compare seasons. For each test a statistical significance level of 0.05 was used.

\section{pH model}

A nonlinear regression analysis was performed to quantify and compare $p H$ curves. Two models were tested: type 1 contained a single exponential function (Eq. (3.1)), and type 2 contained a double exponential function (Eq. (3.2)).

$$
\begin{aligned}
& p H=A-d \cdot e^{-B \cdot t} \\
& p H=A-d_{1} \cdot e^{-B \cdot t}-d_{2} \cdot e^{-C \cdot t}
\end{aligned}
$$

In Eqs. (3.1) and (3.2), the $A$ represents the asymptote, which is the $\mathrm{pH}$ at $t=\infty$ h. The $d, d_{1}$, and $d_{2}$ represent the difference between $A$ and the initial $p H$, so that the initial $p H=A-d$, or initial $p H=A-d_{1}-d_{2}$. The exponential terms $B$ and $C$ represent the $p H$ increase. In Eq. (3.1), the single exponential term describes the whole $\mathrm{pH}$ curve. In Eq. (3.2), one of the exponential terms describes a fast $p H$ increase at the beginning of each $p H$ curve. This increase can be associated with the emission of $\mathrm{CO}_{2}$. The other exponential term describes a declining $p H$ increase till $t=\infty \mathrm{h}$ that can be associated with the emission of $\mathrm{NH}_{3}$. Finally $t$ was the time in $\mathrm{h}$.

A nonlinear regression was performed with the data of each urine puddle. For each fit, the coefficient $A$ was initially set at $9.2, B$ at $1, C$ at 0.1 , and $d, d_{1}$, and $d_{2}$ at 0.1 . The distribution of each estimated coefficient per puddle was tested for normality with the Shapiro-Wilk test at a statistical significance level of 0.05. The fit was checked using $\mathrm{R}^{2}$ and the Final Prediction Error (FPE) (Keesman, 2011). 
Table 3.3: Measured $p H$ values for each hour $\left(p H_{t_{0}}-p H_{t_{4}}\right)$, with mean $p H$ and standard deviation (SD) per farm, per season, and overall.

\begin{tabular}{ccccccc}
\hline Code $^{*}$ & $\mathrm{~N}$ & $\begin{array}{c}p H_{t_{0}} \\
\text { Mean (SD) }\end{array}$ & $\begin{array}{c}p H_{t_{1}} \\
\text { Mean (SD) }\end{array}$ & $\begin{array}{c}p H_{t_{2}} \\
\text { Mean (SD) }\end{array}$ & $\begin{array}{c}p H_{t_{3}} \\
\text { Mean (SD) }\end{array}$ & $\begin{array}{c}p H_{t_{4}} \\
\text { Mean (SD) }\end{array}$ \\
\hline $\mathrm{S} 1$ & 7 & $8.35(0.52)$ & $8.81(0.42)^{\mathrm{a}}$ & $8.93(0.33)^{\mathrm{a}}$ & $8.94(0.28)$ & $8.91(0.28)^{\mathrm{a}}$ \\
$\mathrm{S} 2$ & 5 & $8.36(0.14)$ & $8.97(0.08)^{\mathrm{a}, \mathrm{b}}$ & $9.06(0.12)^{\mathrm{a}, \mathrm{b}}$ & $9.10(0.15)$ & $9.09(0.13)^{\mathrm{a}, \mathrm{b}}$ \\
$\mathrm{W} 1$ & 7 & $8.14(0.24)$ & $9.07(0.34)^{\mathrm{a}, \mathrm{b}}$ & $9.22(0.32)^{\mathrm{a}, \mathrm{b}}$ & $9.30(0.28)$ & $9.36(0.26)^{\mathrm{b}}$ \\
$\mathrm{W} 3$ & 7 & $8.41(0.22)$ & $9.33(0.22)^{\mathrm{b}}$ & $9.35(0.24)^{\mathrm{b}}$ & $9.27(0.28)$ & $9.18(0.31)^{\mathrm{a}, \mathrm{b}}$ \\
$\mathrm{S}$ & 12 & $8.36(0.39)$ & $8.88(0.33)^{\mathrm{c}}$ & $8.98(0.26)^{\mathrm{c}}$ & $9.01(0.24)^{\mathrm{c}}$ & $8.99(0.24)^{\mathrm{c}}$ \\
$\mathrm{W}$ & 14 & $8.27(0.26)$ & $9.20(0.31)^{\mathrm{d}}$ & $9.28(0.28)^{\mathrm{d}}$ & $9.28(0.27)^{\mathrm{d}}$ & $9.27(0.29)^{\mathrm{d}}$ \\
$\mathrm{All}$ & 26 & $8.31(0.33)^{-}$ & $9.05(0.35)^{-}$ & $9.14(0.31)^{-}$ & $9.15(0.29)^{-}$ & $9.14(0.30)^{-}$ \\
\hline
\end{tabular}

\footnotetext{
* for symbols see Table 3.1 .

a,b statistically significant subgroups between individual farms at a level of 0.05 .

${ }^{\mathrm{c}, \mathrm{d}}$ statistically significant subgroups between seasons at a level of 0.05 .
}

\subsection{Results}

\subsection{1 $\mathrm{pH}$ values}

Table 3.3 shows the mean $p H$ and SD for each hour $\left(p H_{t_{0}}-p H_{t_{4}}\right)$ per farm, per season and overall. Figure 3.2 illustrates the mean curves per farm and season. At farm level, $p H_{t_{0}}$ ranged between 8.14 for farm 1 in winter (W1) to 8.41 for farm 3 in winter (W3). The $p H_{t_{0}}$ values at the farms in summer were about equal to a $\mathrm{pH}$ of 8.35 (S1) and 8.36 (S2). The $p H_{t_{4}}$ was higher and ranged from 8.91 for farm $\mathrm{S} 1$ to 9.36 for farm $\mathrm{W} 1$. Among the individual farms, there were only three cases with a statistically significant difference between two farms. These differences were all related to farm S1, which had the lowest $p H$ values. There were no statistically significant differences between the $p H_{t_{0}}$ values. At season level, the difference of $p H_{t_{0}}$ was only 0.09 . This was not statistically significant. From $p H_{t_{1}}$ till $p H_{t_{4}}$ the $p H$ differences were about 0.3 . These differences were statistically significant.

Figure 3.3 shows the mean $p H$ curve of all measured data along with a bandwidth that was the mean $p H \pm 1.96 \cdot \mathrm{SD}$, and a fit by model type 2 . The mean $p H$ quickly increased in the measurement's first half hour and then slowed. Ninety percent of the difference between $p H_{t_{0}}$ and $p H_{t_{4}}$ was realised within the measurement's first hour, and the $p H$ stabilized afterwards. Figure 3.2 shows that for individual farms, the $p H$ increased, stabilized, or even decreased in $4 \mathrm{~h}$ time. However, some $p H$ values were missing for individual puddles (see Section 3.2.2). The missing data caused some fluctuation in the mean and SD curves, especially in the measurement's last $10 \mathrm{~min}$.

Figure 3.4 shows two scatter plots: $p H_{t_{4}}$ against $p H_{t_{0}}$ and $p H_{\max }$ against $p H_{t_{0}}$. 


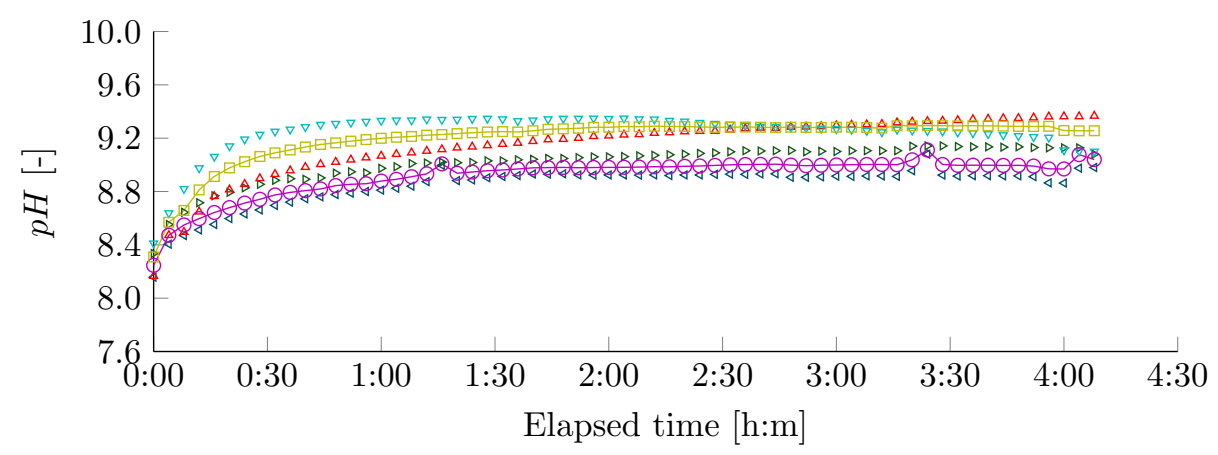

Figure 3.2: The measured $p H$ as mean per farm and season. With farms S1 (๑), S2 (॰), W1 $(\Delta)$, W3 $(\nabla)$, and seasons S $(\odot)$, W $(-\square)$.

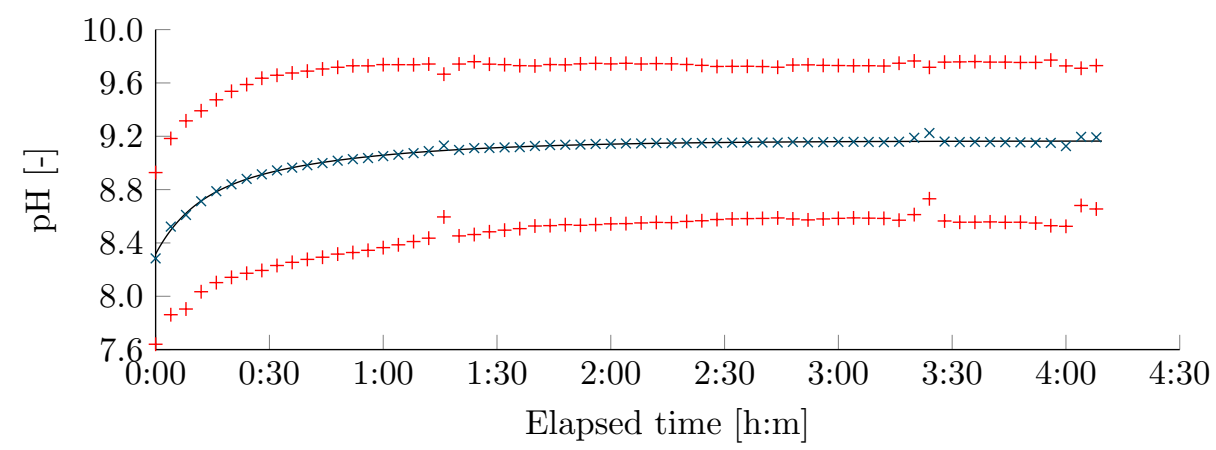

Figure 3.3: The measured overall mean $p H$ curve $(\times)$ with bandwidths of mean $p H \pm 1.96 \cdot \mathrm{SD}(+)$ and fit by type $2(-)\left(p H=9.16-0.38 \cdot e^{-6.63 \cdot t}-0.47 \cdot e^{-1.49 \cdot t}\right)$.

In both cases one puddle showed an extremely high leverage of 0.95 and an extremely high Cook's distance in the linear regression analysis and was therefore excluded from the analyses. In both cases there was no linear relation between $p H_{t_{0}}$, and $p H_{t_{4}}$ or $p H_{\max }$.

\subsection{2 $\mathrm{pH}$ model}

Figure 3.5 shows the boxplots of the estimated coefficients of the regression analysis per puddle according to models type 1 (Eq. (3.1)) and 2 (Eq. (3.2)). For type 1, the $\mathrm{R}^{2}$ values per puddle ranged from 0.26 to 0.99 with a mean of $0.87(\mathrm{SD}=0.20)$. The Final Prediction Error (FPE) ranged from 0.01 to 1.54 with a mean of 0.15 (SD = 0.31). The single exponential curve (Eq. (3.1)) was not able to fit a $p H$ decrease. So for 23 of 26 puddles, the $\mathrm{R}^{2}$ was $>0.80$ and the FPE was $<0.28$. Low $\mathrm{R}^{2}$ values 

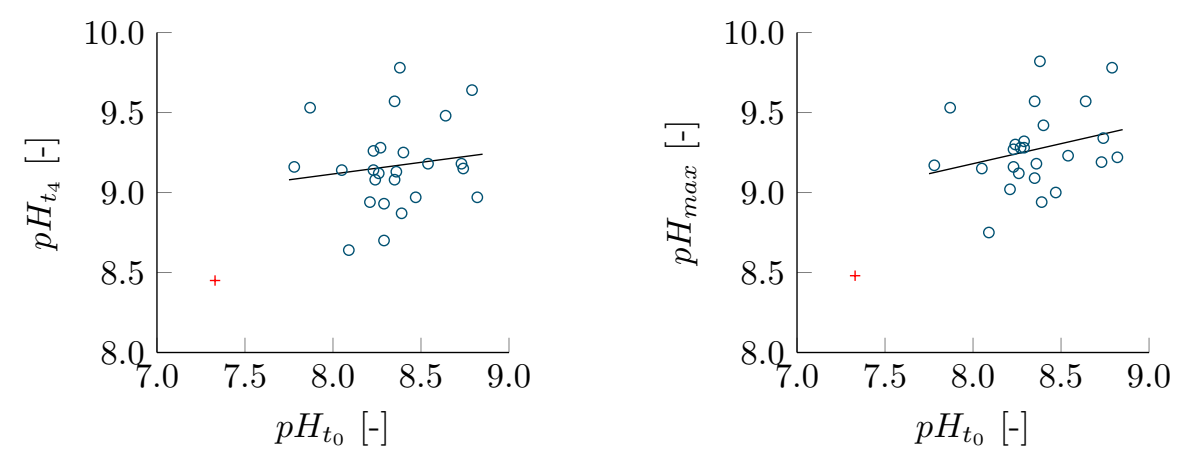

Figure 3.4: Scatter plots of $p H_{t_{4}}$ against $p H_{t_{0}}$ with a linear regression slope of 0.15 and $\mathrm{R}^{2}$ of 0.02 (left) and $p H_{\max }$ against $p H_{t_{0}}$ with a slope of 0.25 and $\mathrm{R}^{2}$ of 0.07 (right). The '+' indicates an outlier with high leverage. This outlier was excluded from the regression analysis.

$(<0.80)$ occurred when a $p H$ curve clearly decreased in $4 \mathrm{~h}$ time; Puddles that only slightly decreased had an $\mathrm{R}^{2}>0.80$ and were represented by a horizontal fit at the end of the $4 \mathrm{~h}$ period. The single exponential curve (Eq. (3.1)) was not able to fit a $p H$ decrease. For the individual coefficients, the median values were $A=9.19, B=1.83$, and $d=0.82$. Normality was confirmed for the distribution of coefficient $A$ but not for $B$ or $d$.

For type 2 , the $\mathrm{R}^{2}$ values per puddle ranged from 0.84 to $>0.99$ with a mean of 0.97 $(\mathrm{SD}=0.04)$. The FPE ranged from $<0.01$ to 0.15 with a mean of $0.04(\mathrm{SD}=0.04)$. Each puddle could be fitted accurately. For the individual coefficients, the median values were $A=9.42, B=4.67, C=0.47, d_{1}=0.76$, and $d_{2}=0.47$. Normality was not confirmed. There were 8 outliers for coefficient $A$ that ranged from -4.68 to 33.18 . Coefficient $d_{2}$ had 7 outliers that ranged from -14.50 to 24.21 . The most extreme outlier was for coefficient $C$, which was 5651 .

Figure 3.3 shows the fit of the measured overall mean $\mathrm{pH}$ curve by type 2 , with $\mathrm{R}^{2}=0.995$ and $\mathrm{FPE}=0.007$. The coefficient estimates (with SE) were: $A=9.16$ $(<0.001), B=6.63(0.257), C=1.49(0.029), d_{1}=0.38(0.011), d_{2}=0.47(0.011)$ that resulted in Eq. (3.3).

$$
p H=9.16-0.38 \cdot e^{-6.63 \cdot t}-0.47 \cdot e^{-1.49 \cdot t}
$$

\subsubsection{Temperature and humidity values}

Table 3.4 shows the mean and SD values for the puddle temperature $\left(T_{l i q}\right)$, air temperature $\left(T_{\text {air }}\right)$, and relative humidity $(R H)$ per farm and season. During summer, 

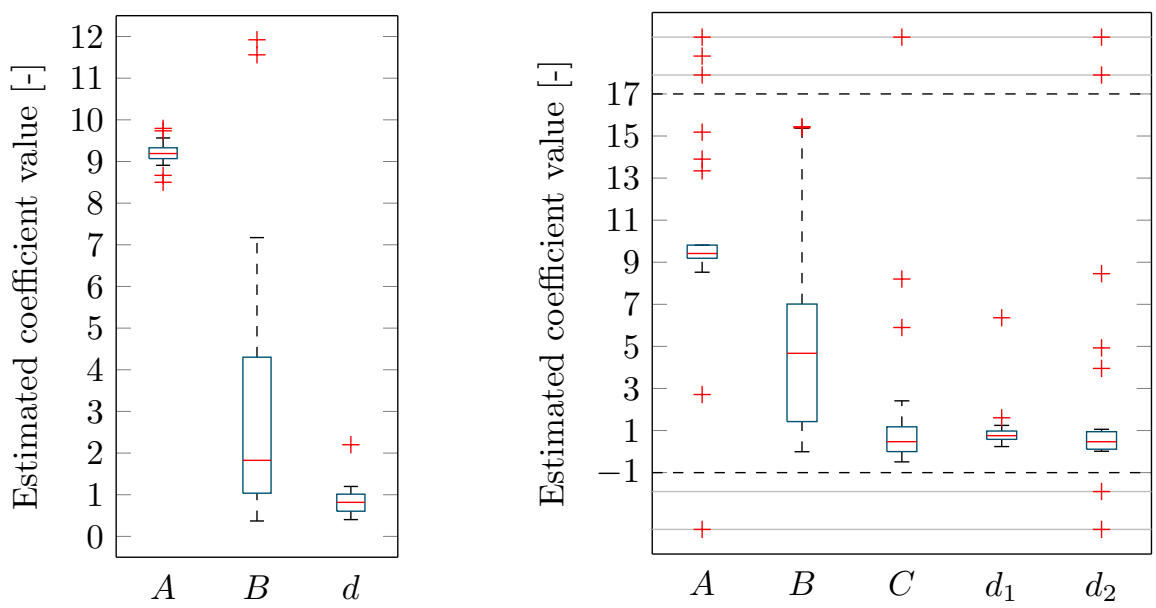

Figure 3.5: Boxplots of the estimated coefficients per puddle, according to model Eq. (3.1) (left), and Eq. (3.2) (right). The '+' indicates outliers.

both $T_{l i q}$ and $T_{a i r}$ were higher than they were in the winter. However, relative humidity was higher in the winter than in the summer. Figure 3.6 shows the temperature curve for $T_{l i q}-T_{a i r}$. For each farm and season, the measurement's initial $T_{l i q}$ was high, but it quickly decreased to a temperature below $T_{\text {air }}$ with a mean difference of $-1.8^{\circ} \mathrm{C}$ at $4 \mathrm{~h}$. Figures 3.7 to 3.8 show the mean $R H, T_{\text {liq }}$, and $T_{\text {air }}$ curves per farm and season, respectively. The temperature measured at farm $\mathrm{S} 2$ was higher for the whole series compared to farm S1, while the humidity was lower at S2 than at S1. During the winter, temperature and humidity were similar at both farms W1 and W3.

Table 3.4: The mean and Standard Deviation (SD) for $T_{l i q}, T_{a i r}$, and $R H$, per farm, per season, and overall.

\begin{tabular}{crrr}
\hline Code $^{*}$ & $T_{l i q}\left[{ }^{\circ} \mathrm{C}\right]$ & $T_{a i r}\left[{ }^{\circ} \mathrm{C}\right]$ & $R H[\%]$ \\
\hline $\mathrm{S} 1$ & $15.6(1.3)$ & $16.8(1.9)$ & $71(8)$ \\
$\mathrm{S} 2$ & $18.9(1.5)$ & $21.6(1.9)$ & $56(11)$ \\
$\mathrm{W} 1$ & $8.7(2.0)$ & $9.2(1.9)$ & $80(8)$ \\
$\mathrm{W} 3$ & $9.1(2.6)$ & $9.6(3.2)$ & $75(14)$ \\
$\mathrm{S}$ & $16.9(2.1)$ & $18.7(3.0)$ & $65(12)$ \\
$\mathrm{W}$ & $8.9(2.3)$ & $9.4(2.7)$ & $77(11)$ \\
All & $12.5(4.6)$ & $13.5(5.4)$ & $72(13)$ \\
\hline
\end{tabular}

\footnotetext{
for symbols see Table 3.1
} 


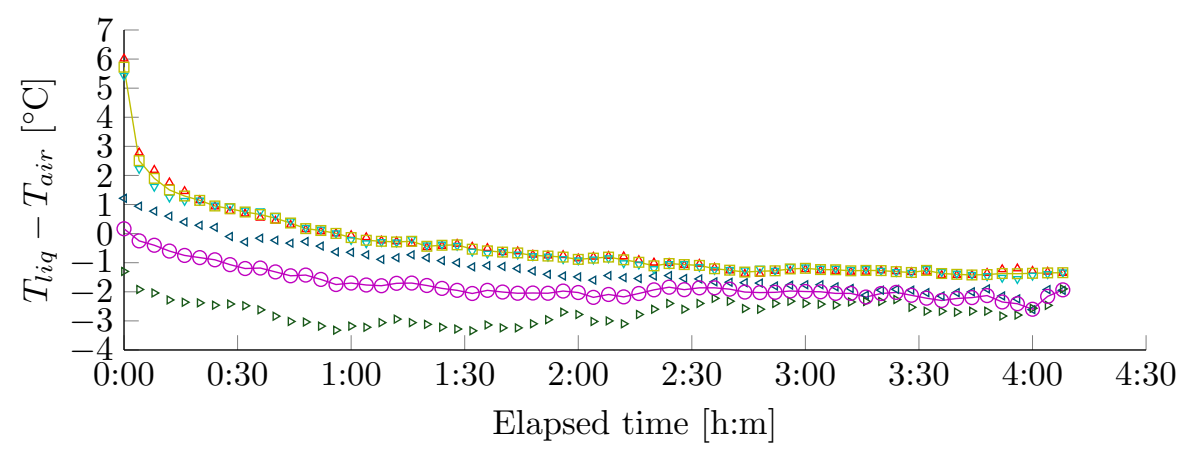

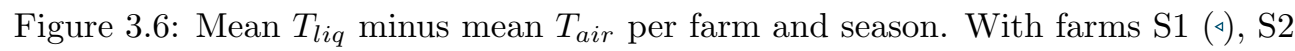
$(\triangleright), \mathrm{W} 1(\Delta), \mathrm{W} 3(\nabla)$, and seasons $\mathrm{S}(\bullet-), \mathrm{W}(-\square)$.

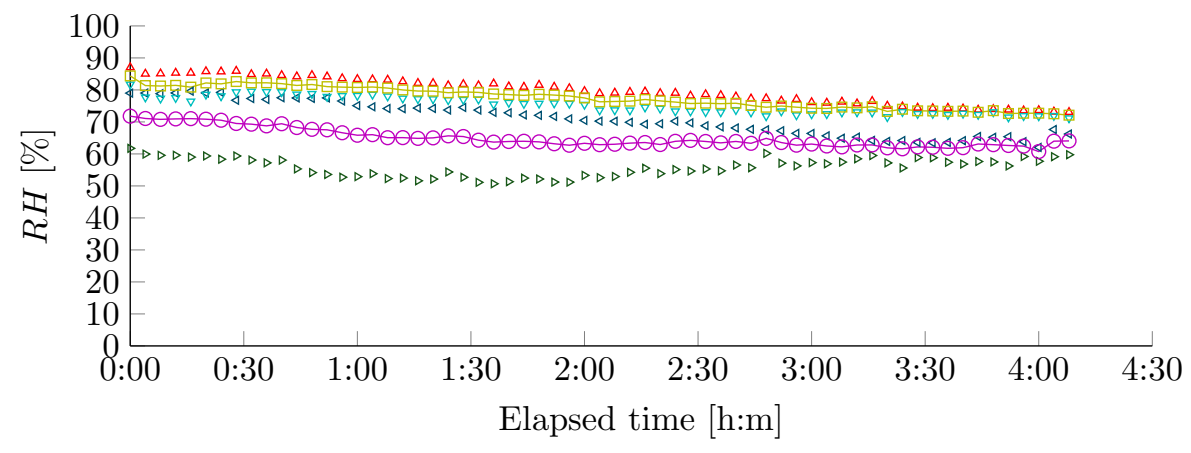

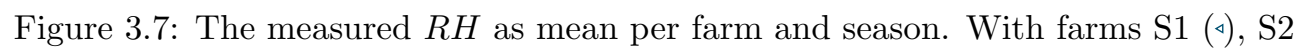
$(\triangleright), \mathrm{W} 1(\Delta), \mathrm{W} 3(\nabla)$, and seasons $\mathrm{S}\left(\odot^{-}\right), \mathrm{W}\left(\bullet^{-}\right)$.

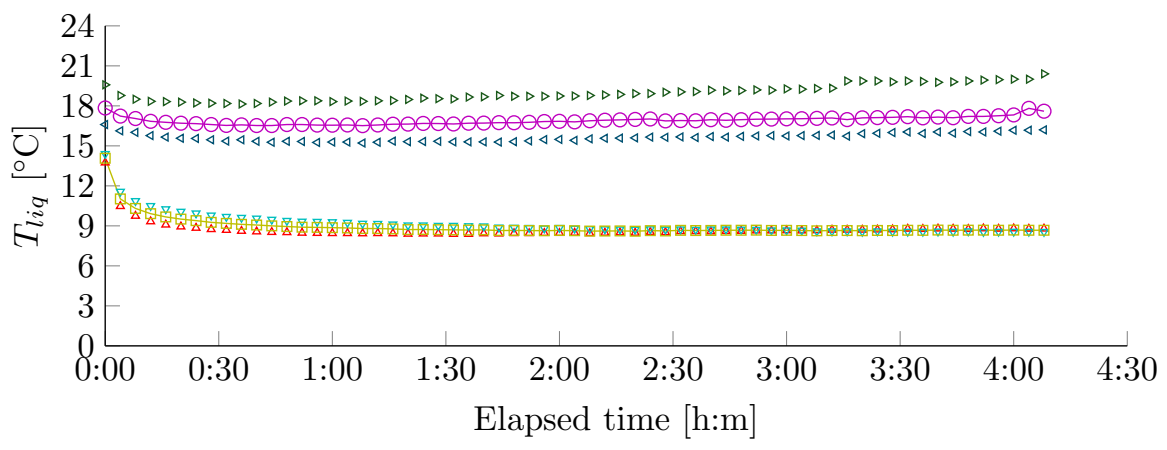

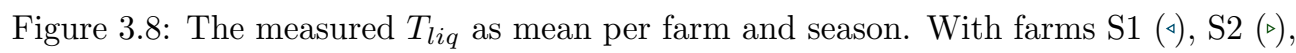
$\mathrm{W} 1(\Delta), \mathrm{W} 3(\nabla)$, and seasons $\mathrm{S}(-\circ), \mathrm{W}\left(-{ }^{-}\right)$. 


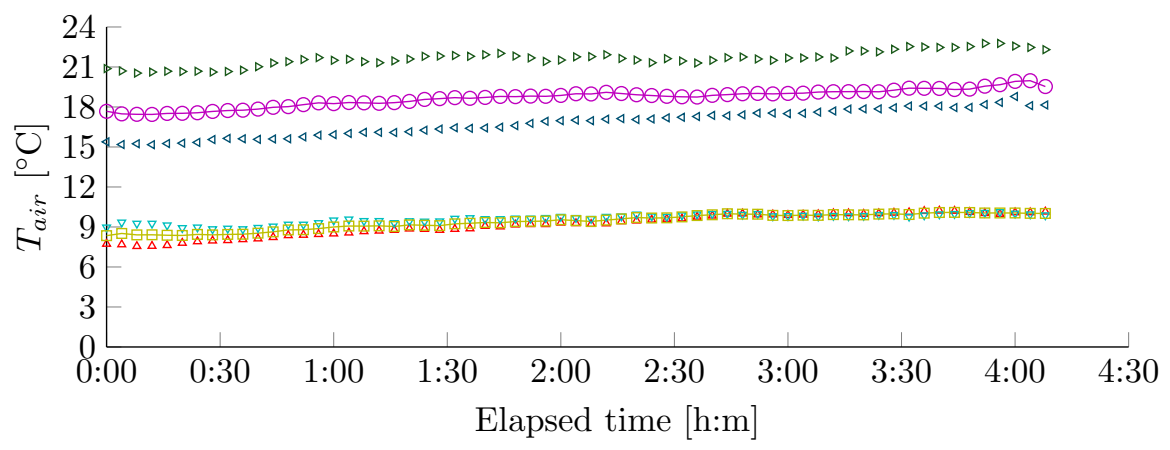

Figure 3.9: The measured $T_{\text {air }}$ as mean per farm and season. With farms S1 (๑), S2 $(\triangleright), \mathrm{W} 1(\Delta), \mathrm{W} 3(\nabla)$, and seasons S $(\bullet-), \mathrm{W}(-\square)$.

\subsection{Discussion}

\subsubsection{Measurements}

Until now, $p H$ values of animal waste have been explored only under controlled lab conditions with collected cow or pig urine, faeces or slurry (Chaoui et al., 2009; Cortus et al., 2008; Monteny, 2000; Sommer \& Sherlock, 1996). Even though real urine or faeces was used in these experiments, measurement of a collected urine or faeces sample in a lab may be different from a fresh urine puddle on the floor of a commercial dairy cow house for two reasons. First, lab experiments do not immediately start to measure the $p H$ and temperature of urine just excreted by a cow at $t=0 \mathrm{~s}$. To measure in a fresh urine puddle at $t=0 \mathrm{~s}$, however, is possible inside a cow house. Second, the lab experiments do not take into account the possible effect on urine $p H$ of fresh faeces, walking cows, the deterioration of the floor and other factors related to a commercial cow house. By measuring inside commercial houses, we accounted for all these effects.

In this study time $t=0 \mathrm{~s}$ indicated when the $p H$ sensor was inserted into a fresh urine puddle and a measurement was started. Ideally, this was when a cow had just finished urinating. In practise, however, the start of the measurement depended on the behaviour of the cows around the puddle, specifically if the cow immediately moved away from the puddle after urinating or if the area around the puddle was free of neighbouring cows. In the latter case, we sometimes had to force cows out of the area to take measurements. This occasionally took some time and delayed the measurement process. However, we tried to put the sensor in the puddle as soon as the cow had finished urinating. This was generally within $15 \mathrm{~s}$. In some extreme cases, this time was estimated to be a minute maximum. Because, on average, we quickly placed the $p H$ sensor in the puddle, we believe these few extreme cases had a negligible effect on 
the results.

It is difficult to measure the surface $p H$ of a thin layer of fluid (Chaoui et al., 2009). We did not find other research that measured the $p H$ of a thin layer like a urine puddle at a concrete floor. In this study the puddle depth was roughly estimated to be about $2 \mathrm{~mm}$ on average. Therefore, we expected that it would be impossible to distinguish between different depths. In addition, we assumed that the measured $p H$ of a puddle represents the 'top layer' of a puddle. According to the manufacturer, the sensor used was able to measure thin fluid layers. This was confirmed by a preliminary test where thin puddles of calibration buffer solutions were measured correctly.

The concrete floor may affect the urine $p H$, the $p H$ measurement, or both. We did not measure the floor $p H$, so we could not distinguish between floor $p H$ and puddle $p H$. In The Netherlands, concrete is the most widely used floor material. All floors in this study were already in use for several years, meaning that among others the $p H$ was settled down and that they were a good representation of concrete floors in practice. Therefore, the possible floor effect on $p H$ is considered to be no problem, as it is part of the commercial dairy cow houses setting, which was the purpose of our research. The same holds for faeces. Most likely faeces affects the urine $p H$, but here as well our measurements represented the situation in practice.

In conclusion, we measured the puddle $p H$ with an accuracy of at least 0.1 inside commercial dairy cow houses. This was accurate enough for our objectives given the observed amount of $p H$ variation.

\subsection{2 $\mathrm{pH}$ values}

At farm level, the current study showed end $p H$ values of 8.9 to 9.4 at $t=4 \mathrm{~h}$. These values were slightly lower than the end values of 9.4 to 9.6 found by Monteny (2000), even though Monteny hypothesized that $p H$ measured in commercial dairy cow houses may be higher than that measured in a lab. Furthermore, the $p H$ curves of our study showed some similarities to those of Monteny's (2000) lab experiments. However, the lab results reached end $p H$ values later in time, after approximately $6 \mathrm{~h}$ to $10 \mathrm{~h}$ or even later in some cases. We measured $p H$ of fresh urine on the floor in an open area of a cow house, whereas Monteny (2000) measured $p H$ and gas concentrations of collected urine in controlled and closed containers inside a lab. Monteny (2000) extracted air, and thus $\mathrm{NH}_{3}$ and $\mathrm{CO}_{2}$, from the container at a controlled flow rate of $0.931 \mathrm{~min}^{-1}$. Considering this, $\mathrm{NH}_{3}$ and $\mathrm{CO}_{2}$ concentrations in the container's head-space may have developed faster than the flow rate could remove them, resulting in a lower concentration difference between $\mathrm{NH}_{3}$ and $\mathrm{CO}_{2}$ in the head-space compared to the sample's top layer. A lower concentration difference will result in a slower or even a halted emission process, and thus a slower change of $p H$. This concentration difference was confirmed by Chaoui et al. (2009) in similar lab experiments with slurry 
in closed jars. They found a six-fold increase of $\mathrm{NH}_{3}$ concentration in the head-space when they opened the jars. A repeated comparison experiment with a closed jar and a jar with a $\mathrm{CO}_{2}$-absorbing trap showed that the $\mathrm{NH}_{3}$ emission almost doubled in the jar with the trap. The $\mathrm{CO}_{2}$ emission increased both the surface $p H$ and the $\mathrm{NH}_{3}$ emission (Chaoui et al., 2009; Hafner et al., 2013). In conclusion, we argue that a closed measurement method strongly influences the $\mathrm{CO}_{2}$ and $\mathrm{NH}_{3}$ concentration both in the head-space and in the sample. As such, the $p H$ is also affected. Therefore, lab measurements do help to better understand specific processes, but the $p H$ behaviour in closed lab measurements are not representative for a farm. The $p H$ increase is faster in open air than in lab experiments with pre-collected urine or faeces in jars.

\subsubsection{Dynamic behaviour $\mathrm{pH}$}

The $p H$ values in this study showed large variation in behaviour and the mean $p H \pm 1.96 \cdot$ SD showed a wide range. For some individual puddles the $p H$ increased over the full $4 \mathrm{~h}$ period, some decreased, and some were already stable after $1 \mathrm{~h}$. In addition, the results in Fig. 3.4 show no linear relation between $p H_{t_{0}}$ and $p H_{t_{4}}$ or $p H_{\max }$. The exact causes for these varying $p H$ curves remain unclear. A possible cause may be the floor condition or cleanliness, but based on this study we cannot draw a conclusion. The large variation was confirmed by the estimated exponential coefficients $B$ and $C$ that showed considerable variation between puddles (Fig. 3.5). Furthermore, each coefficient had outliers, and normality was confirmed in only one case. The pairwise seasonal comparisons showed that there was a statistically significant difference between the summer and winter measurements, although for farm 1, which was measured both in summer and in winter, the difference was only significant at $t=4 \mathrm{~h}(p<0.05)$. Additionally, the SD varied from 0.08 to 0.52 between farms (Table 3.3). These limited statistical differences and large variation in SD suggest that $p H$ values may vary within a farm among cows as much as between farms and between seasons.

There were no statistically significant differences between farms for the initial $p H$. This suggests that the effect of cow management related factors, like feed, was negligible. The $p H$ differences in consecutive parts of the curve may therefore be related to the local floor conditions like the presence of faeces. Based on this study we cannot draw a conclusion at this point.

Two $p H$ models were evaluated on their explanatory value for the $\mathrm{pH}$ data observed. Both models were able to fit the data. Model type 2 (Eq. (3.2)) was more accurate for each individually measured $p H$ curve and its coefficients could be related to the chemical processes. However, the estimated coefficients showed quite some variance and extreme, unreliable values compared to type 1 (Fig. 3.5). In contrast, model type 1 (Eq. (3.1)) was more simple with only one exponential term and it was more stable than type 2 as can be concluded from the smaller variance in the estimated coefficient 
values (Fig. 3.5). However, type 1 was not able to fit the behaviour of each $p H$ curve. For three individual, decreasing curves type 1 had a low $\mathrm{R}^{2}$. For the remaining 23 puddles, type 1 showed fit-results similar to type 2 , but type 1 was not always able to correctly fit the fast $p H$ increase in the first hour.

From the literature only one other empirical $p H$ model was found that contains an exponential part and a linear part (Monteny, 2000). However, we argue that the use of a linear part in Monteny's model may not be correct because the processes of urea conversion, $\mathrm{CO}_{2}$ and $\mathrm{NH}_{3}$ emission, and thus $p H$ change, do not become linear in time (Blanes-Vidal et al., 2009; Chaoui et al., 2009). Nevertheless, the $p H$ change is not infinite because the source, being the urine puddle, depletes. When all the water evaporates from a puddle, all $\mathrm{CO}_{2}$ and $\mathrm{NH}_{3}$ are also most likely emitted because the mass transfer coefficient of $\mathrm{NH}_{3}$ is slightly larger than that for water (Cortus et al., 2008), and $\mathrm{CO}_{2}$ emits even faster than $\mathrm{NH}_{3}$ (Chaoui et al., 2009). The substances remaining on the floor will be small amounts of condensed salts, enzymes, minerals, vitamins or hormones. The absence of a puddle containing urea, $\mathrm{CO}_{2}$ or $\mathrm{NH}_{3}$, will end the $p H$ change. During the measurements, some of the puddles evaporated within $4 \mathrm{~h}$. Therefore, we expect that the end $p H$ of a urine puddle will be similar to the end values measured within this study.

The currently used $\mathrm{NH}_{3}$ emission model for dairy cow houses in The Netherlands makes use of a static $p H$ value as input for the urine puddle simulation. In the reference situation the model simulates 1000 puddles a day that are randomly spread in time and location (Monteny et al., 1998). This is based on 100 cows 10 urinations day ${ }^{-1}$. The effect of $p H$ on $\mathrm{NH}_{3}$ emission is substantial as shown in the sensitivity study of Snoek et al. (2014b), a small $p H$ difference in the model input will directly result in a significant change in estimated $\mathrm{NH}_{3}$ emission of a cow house. The current cow house study confirms that $p H$ in a puddle varies over time, on average from 8.31 to 9.14 , with a fast increase in the first hour. Therefore we conclude that a $p H$ curve will better describe the input variable in $\mathrm{NH}_{3}$ emission of dairy cow houses, compared to the current situation with a static $p H$ value. To confirm this a new sensitivity analysis needs to be performed followed by a validation study.

Altogether, to conclude this section "Dynamic Behaviour $\mathrm{pH}$ ", we recommend to use the mean $p H$ curve based on the measurements (Figure 3.3) in future $\mathrm{NH}_{3}$ emission modelling. The related fit with model type 2 (Eq. (3.3)) can be used for this purpose.

\subsection{4 $\mathrm{pH}$ in summer vs. winter}

The $p H$ differences between summer and winter may be caused by the temperature of both the puddle and air. In general, when it is warmer, chemical and biological processes accelerate. Because of this, the urea conversion and the $\mathrm{CO}_{2}$ and $\mathrm{NH}_{3}$ 
emission processes were expected to be faster in the warm summer than in the cold winter. As a result, the higher temperatures might increase the $p H$ change. However, the results in Fig. 3.2 do not confirm this. In the summer the $p H$ increased slower than in the winter. We also observed that the $p H$ curves in winter seem to be more dynamic than in the summer. In the winter $p H$ curves seem to decrease or increase towards the $4 \mathrm{~h}$ period, while in summer the $p H$ curves seem to stabilise. Another cause for the $p H$ differences between summer and winter may be the feed of the cows. However, detailed nutritional information was not collected in this study. In studies related to feed in dairy cow houses (Monteny et al., 2002; van Duinkerken et al., 2011), the pooled urine $p H$ values were similar to the initial $p H$ measured in this study $\left(p H_{t_{0}}\right.$; Table 3.3), but these studies did not measure $p H$ time series. In both studies a variety of diets were given to the same group of cows. These diets generated a variety of low and high $U_{0}$, which converts to $\mathrm{CO}_{2}$ and $\mathrm{NH}_{3}$ (Elzing \& Monteny, 1997). In general, a higher $U_{0}$ compared to a lower $U_{0}$ results in higher concentrations of $\mathrm{CO}_{2}$ and $\mathrm{NH}_{3}$. Therefore, the absolute $\mathrm{CO}_{2}$ and $\mathrm{NH}_{3}$ emission $\left[\mathrm{g}\right.$ puddle ${ }^{-1}$ ] and its peaks $\left[\mathrm{g} \mathrm{s}^{-1}\right]$ will be higher. The process of urea conversion to $\mathrm{CO}_{2}, \mathrm{NH}_{3}$ and its emission, in turn, will affect puddle $p H$ (Blanes-Vidal et al., 2009). Despite the absence of detailed feed information, we expect that the feed was the main cause for differences in $U_{0}$ and thus the $p H$ behaviour in time. Therefore, the initial urea concentration $\left(U_{0}\right)$ is probably the main cause for the $p H$ difference between summer and winter, and not the puddle or air temperature.

\subsubsection{Temperature values}

The puddle temperature $\left(T_{l i q}\right)$ dropped quickly to a temperature just below the air temperature $\left(T_{\text {air }}\right)$, with a mean difference of $-1.8^{\circ} \mathrm{C}$ at $t=4 \mathrm{~h}\left(T_{l i q}-T_{\text {air }}\right)$. We assume that the measured $T_{l i q}$ represented the floor temperature $\left(T_{\text {floor }}\right)$ and based on the observed $T_{l i q}$ drop within the first couple of minutes (Fig. 3.8) this $T_{\text {floor }}$ was reached quickly. We did not measure the $T_{\text {floor }}$, so we cannot conclude when exactly $T_{l i q}$ reached the $T_{\text {floor }}$. Based on $T_{l i q}$, we observed that $T_{\text {floor }}$ was higher in the summer compared to the winter, and it even increased during the day in the summer.

The differences in temperature and humidity between farms S1 and S2 in the summer was most likely caused by the time of day. Farm S1 was always measured in the early morning, whereas farm S2 was always measured after farm S1 in the early afternoon. This was caused by the milking scheme of both farms. 


\subsection{Conclusions}

Our objective was to investigate $p H$ values and to study their dynamic behaviour in fresh on-floor urine puddles in commercial dairy cow houses. At farm level we found initial $p H$ values of 8.1 through 8.4 , which increased to 8.9 through 9.4 after $4 \mathrm{~h}$. The $p H$ difference between the summer and winter measurements was statistically significant, but this difference was not confirmed by comparisons at farm level. The $p H$ curves of individual puddles varied substantially and could be fitted by a nonlinear regression model. This model contained correlated coefficients that were able to describe the main, known chemical processes of a urine puddle. However, no linear relation was found between initial and final $p H$ and thus between coefficients. On average, $p H$ quickly increased initially, declined after about $1 \mathrm{~h}$ and became stable around a $p H$ of 9.15 .

In the currently used $\mathrm{NH}_{3}$ emission model for dairy cow houses the $p H$ input variable for the puddle simulation is based on a static $p H$ value. We conclude that a $p H$ curve will better describe this $p H$ input variable. To confirm this a validation study has to be performed. Based on this study, we recommend to use the mean measured $\mathrm{pH}$ curve, represented by Eq. (3.3), as input for the puddle simulation during $\mathrm{NH}_{3}$ emission modelling of dairy cow houses.

\subsection{Acknowledgements}

The authors would like to thank the three involved dairy farmers for their hospitality, cooperation during measurements, as well for their contributions to the discussions of this research. In addition, the authors would like to thank the reviewers for their in-depth feedback, and E. Hooft van Huysduynen, P. van Beveren and B. Vroegindeweij for evaluating the manuscript. Language editing of a near-final draft of the paper was provided by P. Griffith. 
CHAPTER 4

Measurement method for urine puddle depth in dairy cow houses as input variable for ammonia emission modelling

Dennis Snoek

Hans Stigter

Nico Ogink

Peter Groot Koerkamp

Published in CIGR Journal (2015), special issue 18th World Congress of CIGR, 30-36 


\begin{abstract}
Dairy cow houses are a major contributor to ammonia $\left(\mathrm{NH}_{3}\right)$ emission in Europe. To understand and predict $\mathrm{NH}_{3}$ emissions from cubicle dairy cow houses a mechanistic model was developed. Furthermore, a sensitivity analysis was performed on this model to assess the contribution to $\mathrm{NH}_{3}$ emission of each input variable related to a single urine puddle. Results showed that $\mathrm{NH}_{3}$ emission was most sensitive for five puddle-related input variables: $\mathrm{pH}$, depth, initial urea concentration, area and temperature. Unfortunately, cow house data of these variables are scarce due to a lack of proper measurement methods. In this study we focused on a method to assess the urine puddle depth, which can vary between $0.10 \mathrm{~mm}$ and $2.00 \mathrm{~mm}$. Our objective was to develop a measurement method for the urine puddle depth. This method must be capable of assessing puddle depth on the floor in commercial dairy cow houses with a measurement uncertainty of at least $0.1 \mathrm{~mm}$. In this study we compared the balance method as golden standard with the ultrasonic method, which was attached to an XY-table. We measured water puddles in an experimental setup under various conditions. We concluded that the ultrasonic sensor can measure puddle depth and can determine depth differences between puddles both with a measurement uncertainty of $0.1 \mathrm{~mm}$. The comparison between the balance and the ultrasonic method gave a mean difference of $<0.01 \mathrm{~mm}$ ( $\mathrm{se}=0.006$ ) in puddle depth; a Tukey mean-difference plot showed that the two methods were proportional and that there was no systematic bias.
\end{abstract}




\subsection{Introduction}

Ammonia $\left(\mathrm{NH}_{3}\right)$ emission can cause environmental pollution, is a precursor of fine dust particles and is an indirect source of nitrous oxide. To lower $\mathrm{NH}_{3}$ emission, a National Emission Ceiling (NEC) is set for each EU member states. The 2010 NEC set by the European Commission was met by 25 of the $27 \mathrm{EU}$ member states, including the Netherlands. Nevertheless, further mitigation of $\mathrm{NH}_{3}$ emission is necessary in the EU, since the expected NECs set for 2020 will be lower than the NEC 2010. In 2010, $94 \%$ of all $\mathrm{NH}_{3}$ emission from the $27 \mathrm{EU}$ member states originated from agriculture. From agriculture, livestock production systems were responsible for $80 \%$. In the Netherlands, in a typical dairy cow house consisting of a living area with cubicles, slatted floor plus walking and feeding-alleys and a slurry pit underneath the whole house, about $70 \%$ of its $\mathrm{NH}_{3}$ emission is derived from the floor.

To understand and predict $\mathrm{NH}_{3}$ emissions from a dairy cow house a mechanistic model was developed (Monteny et al., 1998). Previously we performed a sensitivity analysis to assess the contribution to $\mathrm{NH}_{3}$ emission of each input variable related to a single urine puddle (Snoek et al., 2012, 2014b). It was concluded that $\mathrm{NH}_{3}$ emission was most sensitive for five puddle-related input variables: $\mathrm{pH}$, depth, initial urea concentration, area and temperature. However, cow house data of these variables are scarce due to a lack of proper measurement methods. In this study we focussed on a method to assess the urine puddle depth, which can vary between $0.10 \mathrm{~mm}$ and $2.00 \mathrm{~mm}$.

Two methods for quantification of puddle depth have been used in earlier research (Aarnink \& Elzing, 1998). First, in an experimental setup a measured $0.5 \mathrm{~kg}$ of urine was poured over an area of $10 \mathrm{~cm} \times 10 \mathrm{~cm}$ clean and fouled slatted floors and the surplus was collected and weighted. Second, the same amount of urine was poured over a clean solid floor area and the wetted area was determined. In both cases the mean depth was the volume divided by the area. Depth values were reported with a resolution of $0.01 \mathrm{~mm}$. It was noted that the depth on the solid floor might have been too shallow since they only used clean floors and that depth has a significant effect on $\mathrm{NH}_{3}$ emission. Another option is to use a laser relief meter to measure distance (Zhixiong et al., 2005). We tested this meter in a preliminary experiment and we concluded that it cannot be used to measure urine puddles, since the laser was not able to measure distance to a liquid.

Our objective was to develop a measurement method for the urine puddle depth variable. The method should be able to assess this variable on the floor in commercial dairy cow houses with a measurement uncertainty of at least $0.1 \mathrm{~mm}$. To do reach this goal, we explored two measurement principles and we performed a series of experiments. 


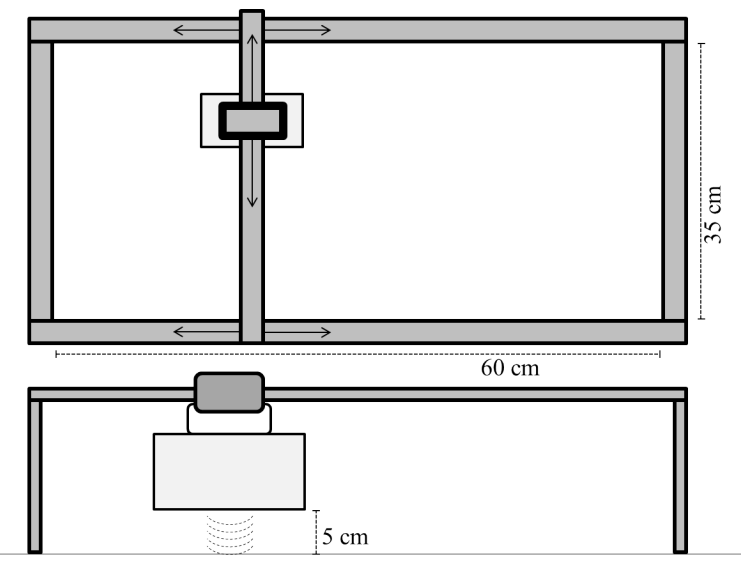

Figure 4.1: Schematic representation of the top and side view of the XY-table with the ultrasonic device and sound waves.

Table 4.1: Conducted experiments (Exp) with a brief description and the used measurement methods.

\begin{tabular}{lll}
\hline Exp & Brief description & Methods \\
\hline 1 & Fixed position in centre of collection tray (Fig. 4.2) & Balance and ultrasonic \\
2 & 4 locations in collection tray (Fig. 4.3) & Balance and ultrasonic \\
3 & 9 locations at floor element (Fig. 4.4) & Only ultrasonic \\
4 & 9 locations at floor element (Fig. 4.4) & Only ultrasonic \\
\hline
\end{tabular}
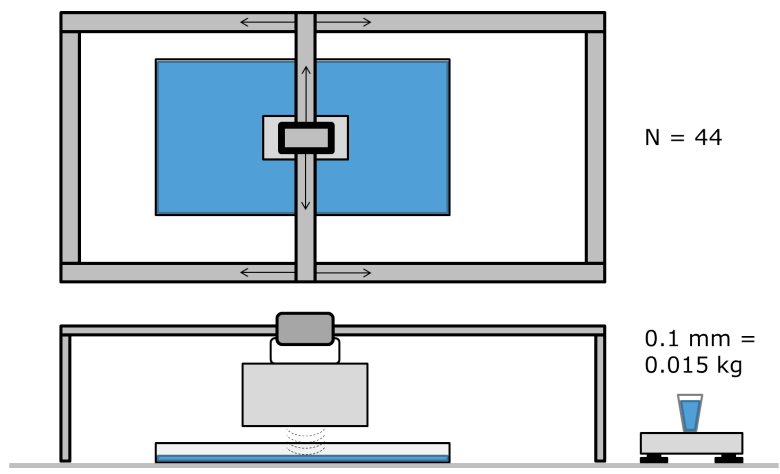

Figure 4.2: Schematic representation of experiment 1, the ultrasonic in the centre of the collection tray 


\subsection{Materials and Methods}

Experiments were conducted to compare two measurement methods to determine puddle depth, being the balance method (Section 4.2.1) as golden standard and the ultrasonic method (Section 4.2.2) to use in commercial dairy cow houses.

\subsubsection{Balance method}

The urine puddle depth was measured in an experimental setup to use as reference. A collection tray (internal dimensions: $500 \mathrm{~mm} \times 500 \mathrm{~mm} \times 25 \mathrm{~mm}$ ) was put on the floor. This tray was filled and emptied with water with a cup. The cup was weighted with a balance (Mettler balance, max. $60 \mathrm{~kg}$ type $\mathrm{KB} 60$, error $=0.01 \mathrm{~kg}$ ) before and after each time that water was poured in or taken out the tray.

The amount of water varied each time. The depth was the volume (calculated from weight / specific weight of the water) divided by the area. A depth of $0.1 \mathrm{~mm}$ was equal to $0.015 \mathrm{~kg}$ of water and $0.001 \mathrm{~kg}$ water was less than $0.01 \mathrm{~mm}$ depth.

\subsubsection{Ultrasonic method}

The urine puddle depth was measured with an ultrasonic device that can measure distance with an accuracy and display resolution of $0.1 \mathrm{~mm}$ and an internal resolution of $0.01 \mathrm{~mm}$. Puddle depth was determined by subtracting the distance to the puddle from the distance to the floor without puddle. To measure puddle depth at various locations of a urine puddle, we first measured the distance to the puddle at various locations and then at exactly the same locations after puddle removal. To return to the exact same location, we operated an XY-table Fig. 4.1. The ultrasonic device was attached to the XY-table and we could move it $35 \mathrm{~cm}$ in $\mathrm{X}$ and $60 \mathrm{~cm}$ in $\mathrm{Y}$ direction. The distance between the ultrasonic and the floor was $5 \mathrm{~cm}$. To remove a puddle we used a water-vacuum-cleaner to vacuum up the puddle from the floor (Kärcher ${ }^{\circledR}$ Window Vac WV 50).

\subsubsection{Validate accuracy ultrasonic method}

Table 4.1 shows an overview of the conducted experiments. Puddle depth values were measured both with the balance (reference) and ultrasonic method at the same time in the experimental setup with collection tray (experiment 1 and 2). In experiment 3 and 4 only the ultrasonic device was used at an experimental setup with the welfare floor 2 (Snoek et al., 2010). In experiments 2, 3 and 4 distance measured with the ultrasonic device was done before, during, and after removal of the puddle. Puddle depth (pd) was determined by subtracting 'distance before' from 'distance to puddle' $(p d 1)$ and by subtracting 'distance after removal' from 'distance to puddle' ( $p d 2)$. 

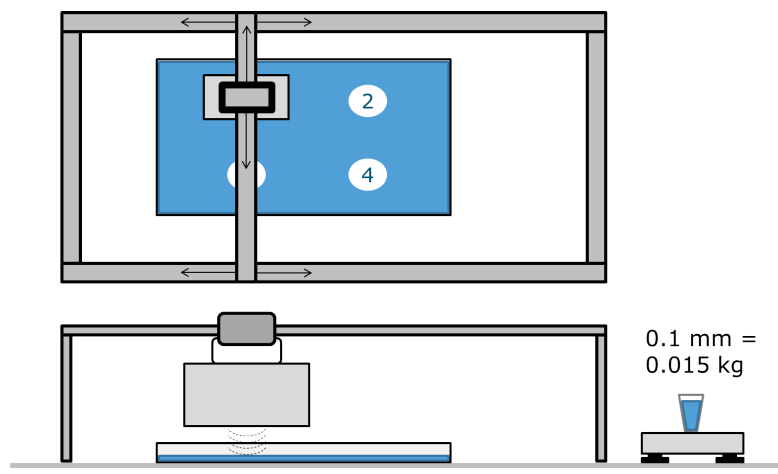

Figure 4.3: Schematic representation of experiment 2, the ultrasonic and the four locations in the collection tray

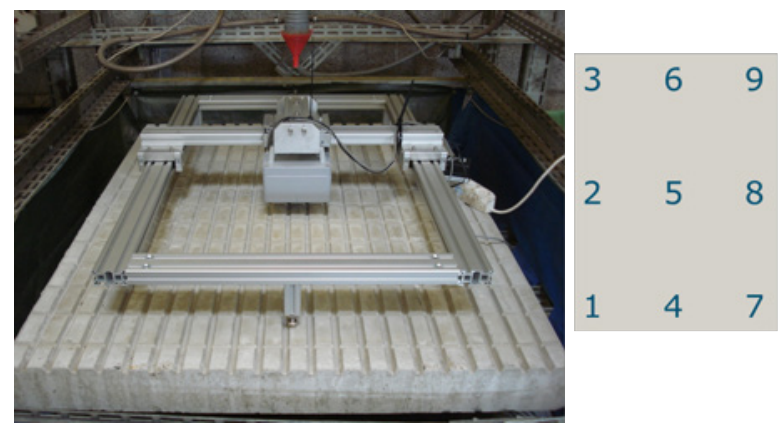

Figure 4.4: Schematic representation (right) of the nine locations at the floor element (left)

\section{Experiment 1}

The ultrasonic was positioned above the centre of the empty collection tray. We gently poured water in and took water out, spread over two measurement series and in varying order. The distance measured with the ultrasonic was saved each time the water level reached a stable level again. For each consecutive step we calculated the increase in depth of the water level determined by both the balance and the ultrasonic method. To assess agreement between the two methods we made a Tukey mean-difference plot, also called Bland-Altman plot (Bland \& Altman, 2010). Except for five steps we tried to keep the added or removed amount of water small to generate depth changes around $0.1 \mathrm{~mm}$. The five steps with a larger amount elevated the water level in the tray to cover a wider measurement range. 


\section{Experiment 2}

The collection tray was filled with about $1 \mathrm{~kg}$ of water to be sure to have a levelled base. Four locations were defined (Fig. 4.3). The ultrasonic was positioned at location 1 of 4. Then the ultrasonic was moved in a sequence consisting of three times a fixed order, being location 1-2-3-4, and three times in a random order. We measured each location according to the sequence before, during, and after puddle removal. The $p d 1_{\text {tray }}$ and $p d 2_{\text {tray }}$ were determined (Section 4.2.3). We executed a one sample t-test to check the difference between mean depth by the ultrasonic with the single depth measure by the balance method, and we executed an independent samples t-test for equality of means between fixed and random order movement within $p d 1_{\text {tray }}$ and $p d 2_{\text {tray }}$. We did not test for equality of means between $p d 1_{\text {tray }}$ and $p d 2_{\text {tray }}$ since the amount of water poured in differed from the amount of water taken out.

\section{Experiment 3}

The XY-table with the ultrasonic was put on the welfare floor 2 in our experimental setup (Snoek et al., 2010). Nine locations were defined (Fig. 4.4), excluding gutter area. The ultrasonic was positioned at location 1 of 9 . Then the ultrasonic was moved in a sequence consisting of a fixed order, being location 1-2-3-4-5-6-7-8-9, a random order, and again in fixed and random order for another four times. In total each location was measured 10 times of which five in fixed movement order and five in random order. In this experiment at the experimental setup it was not possible to use the balance method, because the surface area was not fixed and therefore the depth could not be determined in case a known amount of water was applied. We measured each location according to the sequence before, during, and after puddle removal. The $p d 1_{\text {floor }}$ and $p d 2_{\text {floor }}$ were determined (Section 4.2.3). We executed an independent samples t-test for equality of means between fixed and random order movement within $p d 1_{\text {floor }}$ and $p d 2_{\text {floor }}$, and a paired samples t-test for equality of means between $p d 1_{\text {floor }}$ and $p d 2_{\text {floor }}$.

\section{Experiment 4}

Copy of Section 4.2.3, but this time locations 1 to 9 were measured only twice to determine $p d 1_{\text {floor }}$ and $p d 2_{\text {floor }}$, first by fixed and second by random movement order. Location 1 was measured a second time as number 10 in each series. We performed the 2 times 10 series for 3 puddles. We executed an anova to test for differences between the three puddles within $p d 1_{\text {floor }}$ and $p d 2_{\text {floor }}$, and a paired samples t-test for equality of means between $p d 1_{\text {floor }}$ and $p d 2_{\text {floor }}$. 
Table 4.2: $\mathrm{N}$, mean depth $(\mathrm{mm})$ and SE for $p d 1_{\text {tray }}$ and $p d 2_{\text {tray }}$, for fixed and random movement by the ultrasonic, and the depth by the balance method. Followed by the independent samples t-test for equality of means of fixed vs. random movement.

\begin{tabular}{lcccc}
\hline \multirow{2}{*}{ Movement order } & \multicolumn{2}{c}{$p d 1_{\text {tray }}$} & \multicolumn{2}{c}{$p d 2_{\text {tray }}$} \\
$\mathrm{N}$ & Fixed & Random & Fixed & Random \\
Mean depth in mm (SE) & 12 & 12 & 12 & 12 \\
Depth in mm (balance method) $^{\mathrm{a}}$ & $0.10(0.02)$ & $0.10(0.01)$ & $0.18(0.03)$ & $0.18(0.02)$ \\
Mean difference in mm (p-value) $^{\text {a Mean depth of ultrasonic and depth by balance method did not differ significantly }(>0.05)}$
\end{tabular}

Table 4.3: N, mean depth $(\mathrm{mm})$ and SE for $p d 1_{\text {floor }}$ and $p d 2_{\text {floor }}$, for fixed and random movement by the ultrasonic. Followed by the independent samples t-test for equality of means of fixed vs. random and the paired samples t-test for equality of means of $p d 1_{\text {floor }}$ vs. $p d 2_{\text {floor }}$.

\begin{tabular}{llcccc}
\hline & & \multicolumn{2}{c}{$p d 1_{\text {floor }}$} & \multicolumn{2}{c}{$p d 2_{\text {floor }}$} \\
& Movement order & Fixed & Random & Fixed & Random \\
\hline Descriptives & $\mathrm{N}$ & 45 & 45 & 45 & 45 \\
$"$ & Mean depth in mm (SE) & $0.72(0.05)$ & $0.71(0.04)$ & $0.64(0.04)$ & $0.63(0.05)$ \\
Indep t-test & Mean difference in mm & $0.01(0.888)$ & $0.01(0.890)$ \\
Paired t-test & Mean depth in mm (SE) & $0.71(0.03)$ & $0.64(0.03)$ \\
& Mean difference in mm & \multicolumn{2}{c}{$0.08(<0.001)$} \\
\hline
\end{tabular}

Table 4.4: N, mean depth $(\mathrm{mm})$ and $\mathrm{SE}$ for $p d 1_{\text {floor }}$ and $p d 2_{\text {floor }}$, for puddles 1 to 3 by the ultrasonic. Followed by the one-way ANOVA for equality of means between the puddles (homogeneous subsets), and the paired samples t-test for equality of means of $p d 1_{\text {floor }}$ vs. $p d 2_{\text {floor }}$.

\begin{tabular}{lcccccc}
\hline & \multicolumn{3}{c}{$p d 1_{\text {floor }}$} & \multicolumn{3}{c}{$p d 2_{\text {floor }}$} \\
Puddle & 1 & 2 & 3 & 1 & 2 & 3 \\
\hline $\mathrm{N}$ & 20 & $10^{\mathrm{a}}$ & 20 & 20 & 20 & 20 \\
Mean depth [mm] (SE) & $0.68(0.05)$ & $0.65(0.11)$ & $0.71(0.04)$ & $0.68(0.05)$ & $0.66(0.08)$ & $0.70(0.04)$ \\
Homogeneous subsets ${ }^{\mathrm{b}}$ & 1 & 1 & 1 & 1 & 1 & 1 \\
Mean depth [mm] (SE) & & $0.68(0.03)$ & & & $0.68(0.03)$ & \\
Difference [mm] (p-val) & & & $<0.01(1.000)^{\mathrm{c}}$ & & \\
\hline
\end{tabular}

a Missed one series of 10 measurements.

b Subset 1 of $p d 1_{\text {floor }}$ was not the same as subset 1 of $p d 2_{\text {floor }}$.

c Same series of 10 measurements excluded from $p d 2_{\text {floor }}$, to be equal to $p d 1_{\text {floor }}$, so $\mathrm{N}=50$ for both. 


\subsection{Results}

\subsubsection{Experiment 1}

Figure 4.5 shows the Tukey mean-difference plot. Mean difference was $<0.01 \mathrm{~mm}$ (SD $=0.05)$ and $95 \%$ limits of agreements of mean difference was $-0.09 \mathrm{~mm}$ to $0.10 \mathrm{~mm}$. Besides, the $95 \%$ confidence interval for the bias was $-0.01 \mathrm{~mm}$ to $0.01 \mathrm{~mm}$. Regression analysis resulted in $\mathrm{R}^{2}=0.0012(\mathrm{p}=0.78)$, slope $=0.0024(\mathrm{p}=0.78)$ and intercept $=0.0013(\mathrm{p}=0.83)$. In other words, the two methods were proportional to each other with no systematic bias.

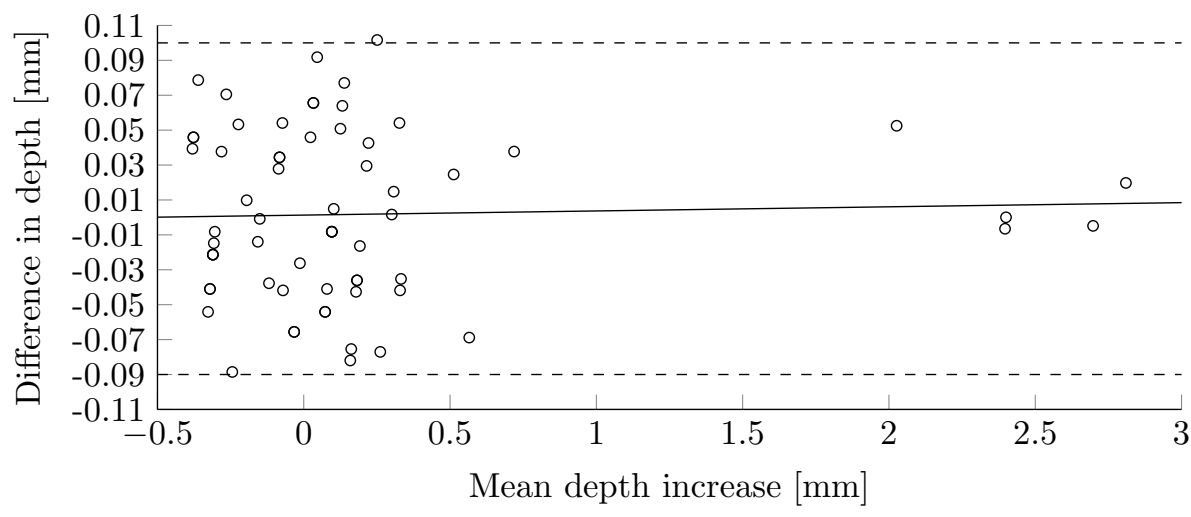

Figure 4.5: Tukey mean-difference plot with $95 \%$ limits of agreement. Difference in depth $[\mathrm{mm}]$ between the balance and ultrasonic method plotted against mean depth increase $[\mathrm{mm}]$ of both methods. $\mathrm{R}^{2}=0.0012(\mathrm{p}=0.78)$, slope $=0.0024(\mathrm{p}=0.78)$, intercept $=0.0013(\mathrm{p}=0.83)$. Mean $\pm 1.96(---)$.

\subsubsection{Experiment 2}

Table 4.2 shows mean depth for $p d 1_{\text {tray }}$ and $p d 2_{\text {tray }}$ with ultrasonic movement in fixed and random order, and the differences. For $p d 1_{\text {tray }}$ the mean difference between fixed and random order was $<0.01 \mathrm{~mm}$ and for $p d 2_{\text {tray }}-0.01 \mathrm{~mm}$. In both cases this difference was not significant $(>0.10)$. The Difference between the mean depth by the ultrasonic and the depth by the balance method did not differ significantly $(>0.05)$.

\subsubsection{Experiment 3}

Table 4.3 shows mean depth for $p d 1_{\text {floor }}$ and $p d 2_{\text {floor }}$ with ultrasonic movement in fixed and random order, and the differences. First, for both $p d 1_{\text {floor }}$ and $p d 2_{\text {floor }}$ the mean difference between fixed and random order was $0.01 \mathrm{~mm}$. In both cases this 
difference was not significant $(>0.10)$. Second, the mean difference between $p d 1_{\text {floor }}$ and $p d 2_{\text {floor }}$ was $0.08 \mathrm{~mm}$ and this time it was significant $(<0.05)$. The mean time it took to measure one location was $12 \mathrm{~s}(\mathrm{SE}=0.3)$.

\subsubsection{Experiment 4}

Table 4.4 shows mean depth for $p d 1_{\text {floor }}$ and $p d 2_{\text {floor }}$ for 3 puddles, and the differences. First, for both $p d 1_{\text {floor }}$ and $p d 2_{\text {floor }}$ the mean differences between the puddles did not differ significantly (each comparison $>0.10$ ). Second, the mean difference between $p d 1_{\text {floor }}$ and $p d 2_{\text {floor }}$ was $<0.01 \mathrm{~mm}$ and this difference was also not significant $(>0.10)$. The mean time it took to measure one location was $11 \mathrm{~s}(\mathrm{SE}=0.4)$.

\subsection{Discussion}

The Tukey mean-difference plot shows that both the balance and the ultrasonic method were proportional with no systematic bias and with low SD and SE. We assumed the balance method to be the golden standard. Therefore, the ultrasonic sensor attached to the XY-table can measure puddle depth with a measurement uncertainty of $0.1 \mathrm{~mm}$.

The readability of the ultrasonic was $0.1 \mathrm{~mm}$. Therefore, a small depth of $0.15 \mathrm{~mm}$ by the balance method as in Table 4.2, may be difficult to measure with the ultrasonic. In this example, the difference between the mean depth measured by the ultrasonic and the depth by the balance method was not significant. In other words, there was no systematic deviation. Additionally, in each conducted experiment in the current study the SE was small that means that there was a good estimate of the mean. To summarise, we conclude that the ultrasonic sensor, attached to the XY-table, can determine depth differences among puddles with an accuracy of $0.1 \mathrm{~mm}$.

The distance between the ultrasonic attached to the XY-table and the floor, was set at about $5 \mathrm{~cm}$. This distance can slightly be adjusted by changing the length of the legs of the table. In general the distance has to be as small as possible, since the distance measurement is based on sound waves and is thus sensitive for temperature changes and air movement. To correct for temperature changes the ultrasonic contains a temperature sensor. We conducted a preliminary experiment by changing the height of the ultrasonic to a fixed, solid, plate from $25.0 \mathrm{~mm}$ to $80.0 \mathrm{~mm}$ with steps of $1.0 \mathrm{~mm}$, with a calliper as reference. It turned out that the measured distance with the ultrasonic was correct for the whole range, but at larger distances the sensor became more sensitive for changing air temperature and air movement compared to smaller distances. Based on the preliminary experiment, and the results in the current paper, we conclude that a $5 \mathrm{~cm}$ distance between floor and ultrasonic is feasible. A shorter distance is better, but then practical problems will arise for measuring puddle depth in dairy cow houses with manure and dirt on the floor. 
Measuring puddle depth with the balance underneath the collection tray may be more accurate than by using the balance on the side and weight a cup with water. But in preliminary test experiments it turned out that the balance was pressed by the weight of the water, resulting in unknown changing distances towards the ultrasonic device. To overcome this changing distance, the tray was put on the concrete floor.

By adding or removing water, the added water or the cup ruffled the surface of the water in the collection tray. We waited until the water was visually stable and the ultrasonic distance measurement gave a stable result.

\subsection{Conclusions}

First, we concluded that the ultrasonic sensor, attached to the XY-table, can measure puddle depth and can determine depth differences between puddles both with a measurement uncertainty of $0.1 \mathrm{~mm}$. The comparison between the balance and the ultrasonic method gave a mean difference of $<0.01 \mathrm{~mm}(\mathrm{SE}=0.006)$ in puddle depth; the Tukey mean-difference plot shows that the two methods were proportional and that there was no systematic bias; and the difference between the ultrasonic and balance method in the movement test above the collection tray was not significant.

Secondly, we concluded that there is no significant difference $(>0.10)$ in the depth measurement by moving the ultrasonic in a fixed or random movement order along the axis of the XY-table.

Thirdly we concluded that evaporation did not influence the measurement. Measurements at the welfare floor 2 (Section 4.3.3) show low SE values, while the measurement period was long $(20 \mathrm{~min})$. The time it took to move to and measure one location was $12 \mathrm{~s}$, so a series of 10 locations takes about $2 \mathrm{~min}$. We expect no significant puddle depth change by water or urine evaporation in this short period of time.

Finally we concluded that the method to remove a puddle worked well in the experimental setup.

\subsection{Recommendations and follow up}

The measurement uncertainty of $0.1 \mathrm{~mm}$ is necessary and sufficient to enable comparison of floor systems and to generate urine puddle depth values for $\mathrm{NH}_{3}$ modelling purposes. Based on the results of this study, the ultrasonic method can be used to measure urine puddle depths in commercial dairy cow houses. Therefore, we will test the puddle removal method in a commercial dairy cow house and we will determine the exact measurement procedure. Then we select floor types, find dairy farmers and design the experiment. Finally we will measure puddle depth values in commercial dairy cow houses. 

CHAPTER 5

\section{Quantification of simulated cow urine puddle areas using a thermal IR camera}

Dennis Snoek

Jan Willem Hofstee

Arjen van Dueren den Hollander

Roel Vernooij

Nico Ogink

Peter Groot Koerkamp

Revised version submitted for Publication 


\begin{abstract}
In Europe, National Emission Ceilings (NEC) have been set to regulate the emissions of harmful gases, like ammonia $\left(\mathrm{NH}_{3}\right)$. From $\mathrm{NH}_{3}$ emission models and a sensitivity analysis, it is known that one of the major variables that determines $\mathrm{NH}_{3}$ emission from dairy cow houses is the urine puddle area on the floor. However, puddle area data from cow houses is scarce. This is caused by the lack of appropriate measurement methods and the challenging measurement circumstances in the houses. In a preliminary study inside commercial dairy cow houses, an IR camera was successfully tested to distinguish a fresh urine puddle from its background to determine a puddle's area. The objective of this study was to further develop, improve and validate the IR camera method to determine the area of a warm fluid layer with a measurement uncertainty of $<0.1 \mathrm{~m}^{2}$. In a laboratory set-up, 90 artificial, warm, blue puddles were created, and both an IR and a colour image of each puddle was taken within $5 \mathrm{~s}$ after puddle application. For the colour images, three annotators determined the ground truth puddle areas $\left(A_{p, G T}\right)$. For the IR images, an adaptive IR threshold algorithm was developed, based on the mean background temperature and the standard deviation of all temperature values in an image. This IR algorithm was able to automatically determine the IR puddle area $\left(A_{p, I R}\right)$ in each IR image. The agreement between the two methods was assessed. The $A_{p, I R}$ underestimated the $A_{p, G T}$ by $2.53 \%$ for which is compensated by the model $A_{p, G T}=1.0253 \cdot A_{p, I R}$. This regression model intercepted with zero and the noise was only $0.0651 \mathrm{~m}^{2}$, so the measurement uncertainty was $<0.1 \mathrm{~m}^{2}$. In addition, the $A_{p, I R}$ was not affected by the mean background temperature.
\end{abstract}




\subsection{Introduction}

In Europe, National Emission Ceilings (NEC) have been set to regulate the emissions of environmentally harmful gases, including ammonia $\left(\mathrm{NH}_{3}\right)(\mathrm{EU}, 2001$; UNECE, 1999), as $\mathrm{NH}_{3}$ deposition leads to eutrophication and acidification of the environment. Although the Netherlands has recently complied with the NEC 2010 (EEA, 2012), $\mathrm{NH}_{3}$ emissions at local and regional levels still lead to overloads in, for example nature reserves (PBL, 2012). In the Netherlands, $94 \%$ of $\mathrm{NH}_{3}$ emissions are produced by the agricultural sector, of which $34 \%$ originates from dairy cow houses and manure storage facilities (EEA, 2012). The typical Dutch cow house consists of a living area with cubicles as well as feeding, drinking and walking areas. The walking areas have a slatted floor, and a slurry pit lies beneath the whole house. The slatted floor contributes about $70 \%$ of a cow house's $\mathrm{NH}_{3}$ emissions and the slurry pit about $30 \%$ (Monteny et al., 1998). With increasing regulatory restrictions on $\mathrm{NH}_{3}$ emissions, there is an urgent need to reduce $\mathrm{NH}_{3}$ emissions from dairy cow houses.

$\mathrm{NH}_{3}$ emissions have long been a concern of the dairy industry, particularly emissions from dairy cow houses, and several mechanistic models have been developed to understand and estimate the $\mathrm{NH}_{3}$ emission process from these houses (Elzing \& Monteny, 1997; Monteny et al., 1998; Montes et al., 2009). However, each of these models uses different model parameters. Moreover, the values for the model input variables vary in commercial dairy cow houses. These two factors cause variations in the estimated $\mathrm{NH}_{3}$ emission. To better understand these variations, the models were recently used in a sensitivity analysis to explore how their parameters and input variables affect the estimated $\mathrm{NH}_{3}$ emission in dairy cow houses (Snoek et al., 2014b). The analysis showed that the five puddle-related input variables: $\mathrm{pH}$, puddle depth, urea concentration, puddle area, and puddle temperature, explained at least $71 \%$ of the variation in the estimated $\mathrm{NH}_{3}$ emissions. The remaining variables were the air temperature and air velocity just above a puddle, the maximum rate of urea conversion or urease activity, and the Michaelis-Menten constant. In addition, the authors observed that the studies that developed or used these models did not have sufficient data on the input variables of commercial dairy cow houses to accurately model $\mathrm{NH}_{3}$ emissions. This was most likely caused by the lack of appropriate measurement methods and by the challenging measurement circumstances in dairy cow houses. If these $\mathrm{NH}_{3}$ emission models are to be improved, accurate data is needed from cow houses at least for the mentioned five variables, which play a major role in accurately determining $\mathrm{NH}_{3}$ emissions in dairy cow houses. In this study we focus on developing an accurate measurement method to quantify the puddle area $\left(A_{p}\right)$ of fresh dairy cow urine puddles on floors inside commercial cow houses.

In the literature, we found three methods to quantify $A_{p}$ in livestock houses. Aarnink et al. (1996) used trained observers to record fouling of the floor in pens of 
rearing and fattening pigs by drawing on paper. They discussed that their method was not very accurate but that the time of day at which the wet area was determined caused the largest variation between measurements. Braam et al. (1997b) used a $1.0 \mathrm{~m}$ $.1 .0 \mathrm{~m}$ frame with equally spaced bars at $0.1 \mathrm{~m}$ that they "placed over a urine pool". They reported $A_{p}$ values of $0.89 \mathrm{~m}^{2}$ to $1.23 \mathrm{~m}^{2}$ at a double-sloped floor in a dairy cow house, but they neither used a reference method nor evaluated the method. Finally, Aarnink et al. (1997) drew a rectangle around the wetted top surface on a slatted floor in a pig house. They then estimated the wet area within this rectangle. Again, the researchers neither used a reference method nor evaluated the method. All three methods used human observation of wetted areas even though it is difficult to observe the area of a fresh urine puddle visually (Aarnink et al., 1996). This is especially difficult in a cow house because a cow urinates on a floor that is often wet and full of other puddles and faeces, both of which are dark coloured. For this reason, we concluded that these methods were not accurate enough to quantify $A_{p}$ values inside commercial dairy cow houses or to use as reference method.

While no method has yet been developed to accurately measure $A_{p}$ values, thermal IR imaging shows potential. Thermal IR cameras have successfully been applied in agriculture in recent years. They have been used to detect and quantify apple scab (Belin et al., 2013), to assess body condition scores of cows (Halachmi et al., 2013), to develop a teat sensing system for robotic milking (Ben Azouz et al., 2015), and to screen seeds (Dumont et al., 2015). A thermal IR camera has also successfully been tested in a preliminary study inside commercial dairy cow houses to distinguish a fresh urine puddle from the background to determine a puddle's $A_{p}$ (Snoek et al., 2014a). A fresh puddle temperature amounts approximately $25^{\circ} \mathrm{C}$ to $35^{\circ} \mathrm{C}$ on the floor. As the puddle ages, it cools to floor temperature, which is ambient temperature (Snoek et al., 2014a). This temperature difference between puddle and background is visible in a thermal IR image. In Snoek et al. (2014a), the IR images were used in a developed image processing model to obtain $A_{p}$ values. However, to obtain correct $A_{p}$ values and to compare puddles, a procedure is needed to set the threshold for background distinction, to calibrate the method, to validate it, and to define its accuracy.

The objective of the current study was to further develop the IR camera method to determine the area of a warm fluid layer with a measurement uncertainty of $<0.1 \mathrm{~m}^{2}$. To do this we analysed IR and colour images of artificial, warm and blue fluid layers. The ground truth of the colour images was used as reference method for the developed IR method. 


\subsection{Materials and Methods}

\subsubsection{Experimental design}

To determine the urine puddle area $\left(A_{p}\right)$, both an IR and a colour image were taken of 90 artificial puddles. Puddle creation was performed by means of a jug and pouring artificial urine (Section 5.2.4) from varying heights. By doing so, the puddles were created in a variety of shapes similar to real urine puddles on floors in commercial dairy cow houses. The experiment was performed in a lab by using three different commercially-available, floor types (Table 5.1). Lab measurements were taken similar to the procedure described by Snoek et al. (2014a); IR and colour images were manually taken $1 \mathrm{~s}$ to $5 \mathrm{~s}$ immediately after an artificial puddle (Section 5.2.4) was created.

Table 5.1: Experimental design with floor type, number of puddles, applied puddle volume, and the floor conditions.

\begin{tabular}{|c|c|c|c|}
\hline Floor type & Puddles [\#] & Volume $[\mathrm{L}]$ & Floor condition \\
\hline 1 solid, levelled, closed floor & 30 & 0.2 to 0.8 & clean and dry \\
\hline 2 commercial slatted floor & 30 & 0.2 to 0.4 & clean and wet \\
\hline 3 commercial grooved floor & 30 & 0.2 to 0.4 & clean and wet \\
\hline
\end{tabular}

\subsubsection{Equipment}

Puddles images were taken with a thermal infrared (IR) camera (FLIR ${ }^{\circledR}$ SC660; FLIR Systems, Inc. USA), with a FLIR P/N T197089, $24^{\circ} \times 18^{\circ}, f=35 \mathrm{~mm}$ lens. This camera took IR images in ${ }^{\circ} \mathrm{C}$ of $640 \cdot 480$ pixels with an accuracy of $\pm 1{ }^{\circ} \mathrm{C}$. The camera simultaneously took colour images of $2048 \cdot 1536$ pixels with an additional lens, positioned right above the IR lens. The camera was mounted on a trolley at a fixed height of $1.90 \mathrm{~m}$ and at a $35^{\circ}$ angle (Fig. 5.1, Snoek et al., 2014a). Both the IR and colour images were saved as JPEG images. The IR images were converted to .csv files of $640 \cdot 480$ cells containing the temperature values in ${ }^{\circ} \mathrm{C}$ with 1 decimal. This was done with the ThermaCAM ${ }^{T M}$ Researcher Pro 2.10 (FLIR Systems, Inc. USA).

\subsubsection{Image calibration}

A calibration method was applied to know the real-world area size in an image, and to correct for camera angle and height. First, both an IR and a colour image were taken of a $1.0 \mathrm{~m} \cdot 0.6 \mathrm{~m}$ aluminium plate that was located in the centre of the IR camera's field of view (Fig. 5.1). A point coordinates calibration was then performed with Vision Assistant 2013 (National Instruments Corporation, USA). To do this, we manually 


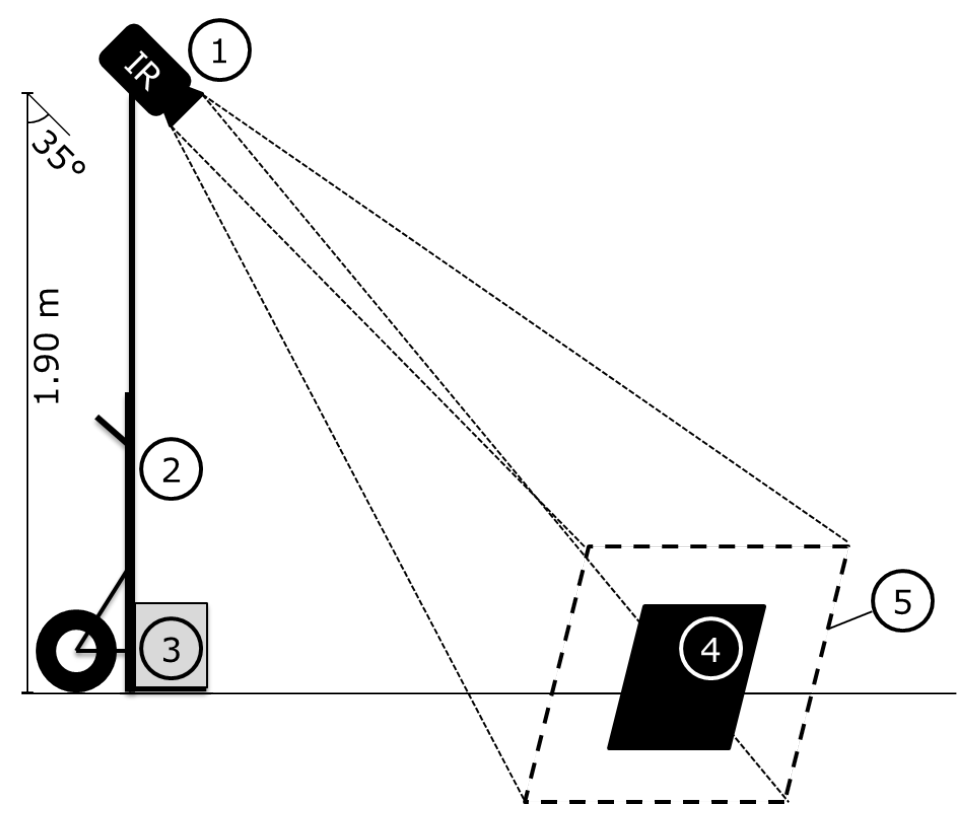

Figure 5.1: Schematic representation of the IR camera (1) on a trolley (2), with dead weight (3) and an aluminium rectangle plate (4) for calibration at the indicated view (5) (Snoek et al., 2014a).

selected the plate's four corners in an image, entered the real-world distances, and saved the information in a calibration file. This technique, known as orthorectification, is often used to remove the effects of image perspective (tilt) and relief (terrain). It is applied to create a planimetrically correct image from images collected by an airplane (Meo et al., 2012) or satellite (Leprince et al., 2007). Each time the trolley was built up, this calibration method was executed.

Two other calibration methods were tested to automatically calibrate each image. One method used the information from the image itself, for example the size of a floor design element. The other method used the information of a known object that was inserted into each image. From preliminary tests we concluded that these methods did not work for our purpose.

\subsubsection{Artificial puddle}

The artificial puddles consisted of warm water of approximately $30^{\circ} \mathrm{C}$, a temperature similar to fresh dairy cow urine (Snoek et al., 2014a), to which a blue dye had been added (Americolor Royal Blue 102). Immediately after the artificial puddle was formed, an IR and a colour image were taken. 


\subsubsection{Ground truth}

The colour images of the blue puddles were used to determine the ground truth puddle area $\left(A_{p, G T}\right)$. Each colour image was aligned with the corresponding IR image. The blue puddle pixels in the colour images were then selected and labelled 'puddle' by a manually set threshold based on the Hue-Saturation-Intensity (HSI) colour space. The number of puddle-pixels was counted, and the $A_{p, G T}$ was determined by using the real-world area per pixel from the calibration file (Section 5.2.3). The threshold was set by three persons individually, so-called annotators, and the outcomes were averaged to calculate the final $A_{p, G T}$. The annotators were able to recognise the complex floor designs and patterns in the images to correctly select the puddle pixels. In other words, each annotator determined for each pixel in each image whether it represented the puddle or not. Three annotators were used instead of one to obtain accurate $A_{p, G T}$ values.

\subsubsection{IR-model}

The IR images of the warm puddles were used to determine the IR puddle area $\left(A_{p, I R}\right)$. For each pixel in each IR image, the developed IR algorithm (Eqs. (5.1) to (5.3)) determined whether it represented the puddle or not. The working principle of the algorithm was based on temperature differences. With a threshold, the pixels with high temperatures were selected and appointed to be puddle, while pixels with low temperatures were not taken into account. The number of puddle pixels was counted, and the $A_{p, I R}$ was determined by using the real-world area per pixel from the calibration file (Section 5.2.3). The algorithm was developed in LabVIEW ${ }^{\text {TM }}$ 2013, version 13.0f2 (National Instruments Corporation, USA); Matlab ${ }^{\circledR}$ R2015b (Mathworks, USA) was used for the analyses.

The IR algorithm selected the pixels of a warm puddle in an IR image by a temperature based threshold. An adaptive threshold was used that was similar to the method described by Gonzalez \& Woods (2008) and Bac et al. (2014) to manage temperature differences between IR images. The maximum temperature $\left(T_{\max }\right)$ in the IR image was considered to be the maximum threshold $\left(t h l d_{\max }\right)$, whereas the minimum threshold $\left(t h l d_{m i n}\right)$ was based on the mean background temperature $\left(\overline{T_{\text {background }}}\right)$ and the threshold shift term $a$. This $\overline{T_{\text {background }}}$ represented the floor and was derived from the corner temperatures of the IR image where no puddle was present, according to Eq. (5.1).

$$
\overline{T_{\text {background }}}=\frac{\sum_{i=1}^{400} T_{\text {cor }, i}}{N}
$$


with:

- $\overline{T_{\text {background }}}\left[{ }^{\circ} \mathrm{C}\right]$ mean background temperature of IR image

- $T_{c o r, i}\left[{ }^{\circ} \mathrm{C}\right]$ corner temperatures of $10 \cdot 10$ pixels in corner $i(i=1 \cdots 4)$

- $N[\#]$ total number of corner pixels $(N=4 \cdot 10 \cdot 10=400)$

Subsequently, the $t h l d_{\text {min }}$ was estimated according to Eq. (5.2). thld $d_{\text {min }}$ was shifted from $\overline{T_{\text {background }}}$ by coefficient $a$ and the SD of all temperature values in the IR image. The pixels with a temperature between $t h l d_{\min }$ and $t h l d_{\max }$ were classified as puddle (Eq. (5.3)).

$$
\begin{gathered}
t h l d_{\text {min }}=\overline{T_{\text {background }}}+a \cdot \sigma_{\text {img }} \\
\text { thld } d_{\min }<T_{x, y}<t h l d_{\max }
\end{gathered}
$$

with:

- $\operatorname{thld}_{\min }\left[{ }^{\circ} \mathrm{C}\right]$ minimum threshold

- $a$ [-] the coefficient to control threshold shift from $\overline{T_{\text {background }}}$

- $\sigma_{i m g}\left[{ }^{\circ} \mathrm{C}\right] \mathrm{SD}$ of all IR image temperature values

- $T_{x, y}\left[{ }^{\circ} \mathrm{C}\right]$ temperature of a pixel with coordinates $x$ and $y$

- $\operatorname{thld}_{\max }\left[{ }^{\circ} \mathrm{C}\right]$ maximum threshold, which is $T_{\max }$

\subsubsection{IR model - determination of a}

To determine coefficient $a$, we created a table of 90 by 200 cells containing $A_{p, I R}$ values of each puddle with values for $a$ that ranged from 0.00 to 2.00 (step size $=0.01$ ) With these $A_{p, I R}$ values and the corresponding $A_{p, G T}$, we determined the relative difference $(R D)$ for each $a$ and each puddle (Eq. (5.4)). The $R D$ was averaged per value for $a$, resulting in 200 mean $R D$ values. The $a$ with the lowest mean $R D$ was selected.

$$
R D=\left|\frac{A_{p, G T}-A_{p, I R}}{A_{p, G T}}\right|
$$

\subsubsection{IR-method validation}

$A_{p, G T}$ and $A_{p, I R}$ were assumed to be equal. This was checked with a scatter plot, including the identity line $y=x$. Multiple linear regression analysis was performed 
according to Eq. (5.5). The individual model coefficients were tested with the hypotheses $h_{0}: b_{k}=0$ with $k=0,1$ and 2. Deviation of the identity line $y=x$ was tested with the hypothesis $h_{0}: b_{1}=1$, by using an F-test.

$$
y=b_{0}+b_{1} \cdot x_{1}+b_{2} \cdot x_{2}+e
$$

with:

- $y\left[\mathrm{~m}^{2}\right]$ independent variable $A_{p, G T}$

- $x_{1}\left[\mathrm{~m}^{2}\right]$ independent variable $A_{p, I R}$

- $x_{2}\left[{ }^{\circ} \mathrm{C}\right]$ independent variable $\overline{T_{\text {background }}}$

- $b_{0}, b_{1} \& b_{2}[-]$ model coefficients

- $e$ [-] error

\section{$5.3 \quad$ Results}

\subsubsection{Ground truth}

Figure 5.2 shows the ground truth puddle area $\left(A_{p, G T}\right)$ for all 90 puddles and its Coefficient of Variation (CV) resulting from the three annotators. The $A_{p, G T}$ ranged from 0.11 through $0.95 \mathrm{~m}^{2}$, with the main body of values between 0.1 and $0.5 \mathrm{~m}^{2}$. For almost all puddles, the $\mathrm{CV}$ was smaller than 0.10 , and there was one high value of 0.24. The mean CV was $0.04[-]$ with an SD of 0.04 .

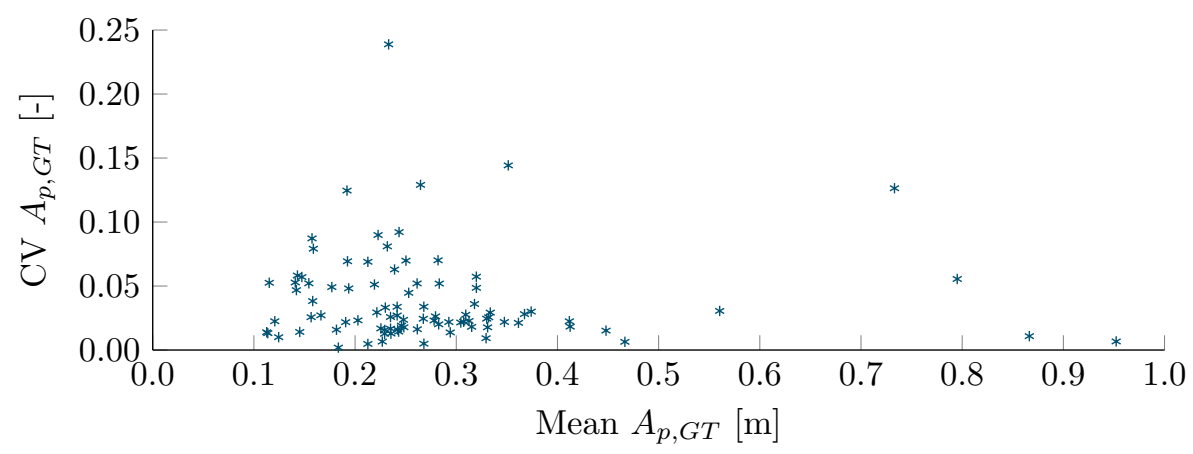

Figure 5.2: Coefficient of Variation (CV) of $A_{p, G T}[-]$, plotted against the mean $A_{p, G T}$ $\left[\mathrm{m}^{2}\right]$. 

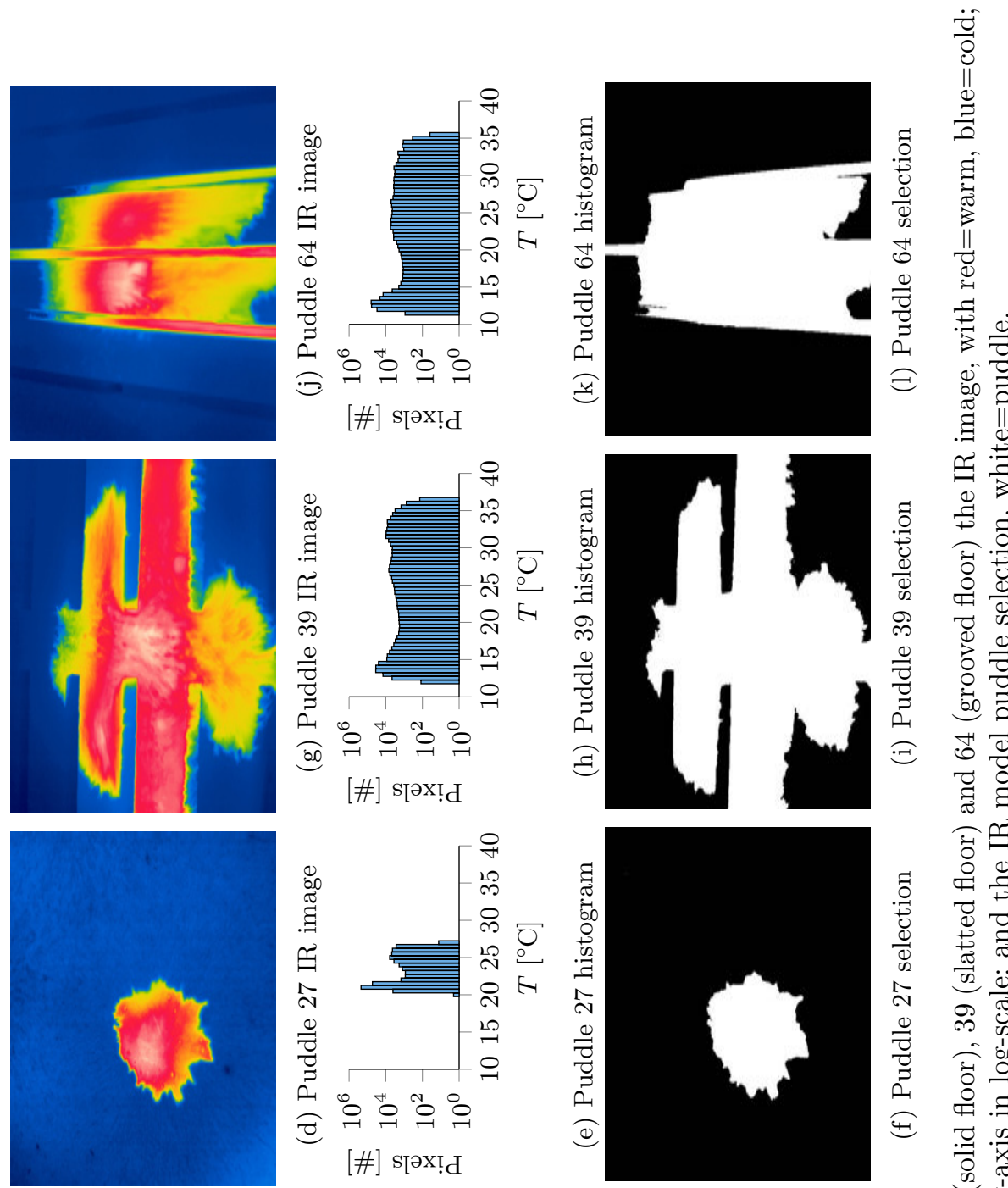

节纴
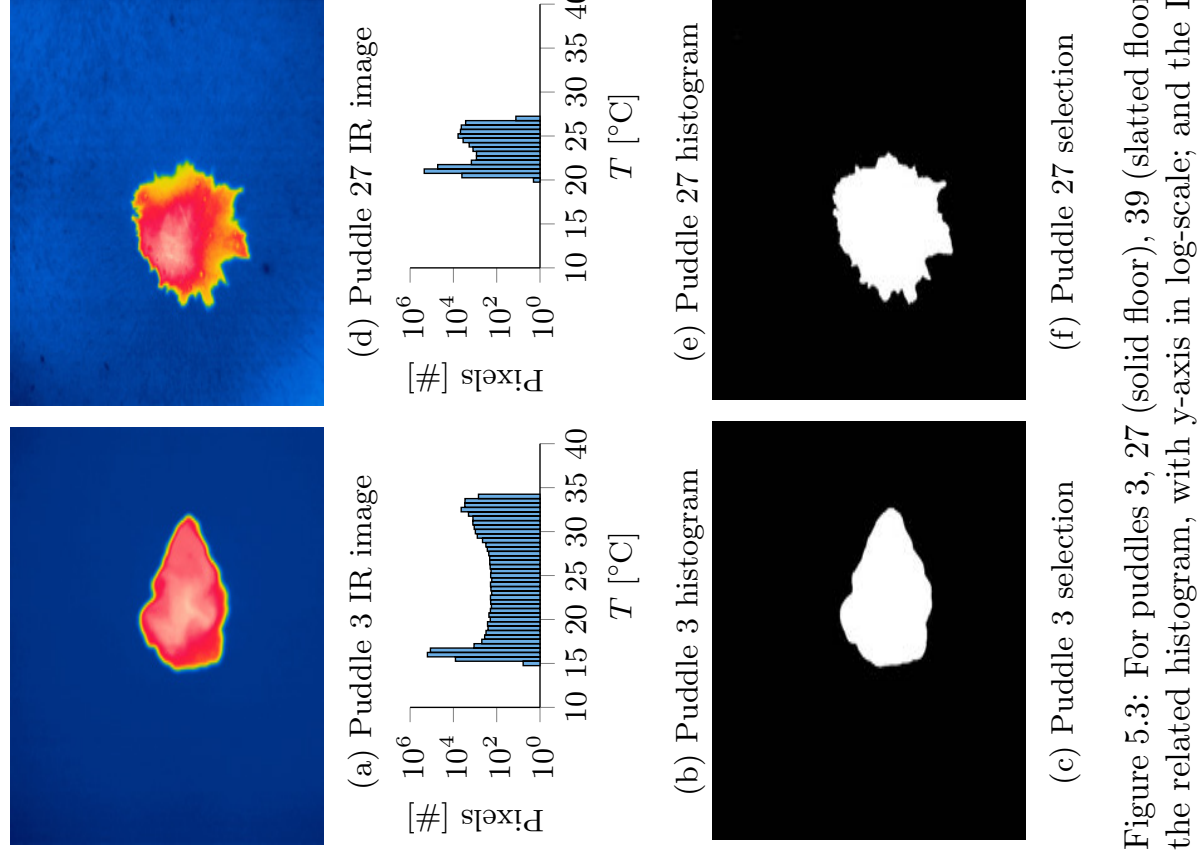


\subsubsection{IR model}

Figure 5.3 shows the IR images, histograms and the puddle selection by the IR model, for puddles 3, 27 (solid floor), 39 (slatted floor) and 64 (grooved floor).

First, in Figs. 5.3a and 5.3d the majority of pixels, the blue colour, represented the lower background temperature $\left(T_{\text {background }}\right)$. This lower temperature was represented by the high peaks on the left side of the related histograms (Figs. 5.3b and 5.3e; y-axis in log-scale). The peaks on the right side in these histograms and the red colour in the IR images represented the puddles that were warmer than $T_{\text {background. For puddle }}$ $3, \overline{T_{\text {background }}}$ was $15.8^{\circ} \mathrm{C}$ (Eq. (5.1)) and the $\mathrm{SD}$ of the image $\left(\sigma_{\text {img }}\right)$ was $3.9^{\circ} \mathrm{C}$. For puddle $27, \overline{T_{\text {background }}}$ was $21.1^{\circ} \mathrm{C}$ and $\sigma_{i m g}$ was $1.1^{\circ} \mathrm{C}$. The minimum threshold $\left(t h l d_{\min }\right)$ will be between the left and right peaks, based on the value for coefficient $a$ (Section 5.3.3). Figures 5.3c and 5.3f show the outcome of the IR model, with the selected pixels in white, which represent the puddle.

Second, the used slatted floor and grooved floor elements were rather small. Therefore we moved the IR camera closer to zoom and consequently the puddles in the IR images covered a larger amount of pixels (Figs. 5.3g and 5.3j). The design characteristics, i. e. the slats (puddle 39) or grooves (puddle 64), of the floors were clearly visible. The related histograms (Figs. $5.3 \mathrm{~h}$ and $5.3 \mathrm{k}$ ) show a temperature range comparable to puddle 3 on the solid floor (Fig. 5.3b). The peaks on the left and right side, however, were less extreme, and temperature values were more widely spread. On the other hand, the peaks were still clearly visible. For puddle $39, \overline{T_{\text {background }}}$ was $13.1^{\circ} \mathrm{C}$ and $\sigma_{i m g}$ was $7.9^{\circ} \mathrm{C}$. For puddle $64, \overline{T_{\text {background }}}$ was $12.4^{\circ} \mathrm{C}$ and $\sigma_{i m g}$ was $6.7^{\circ} \mathrm{C}$. Figures 5.3i and 5.31 show the outcome of the IR model.

A temperature gradient was observed around the edges of a puddle, which is visible in the IR images and the related histograms Fig. 5.3. The centre of a puddle is the warmest location, and the temperature gradually decreases towards the puddle's edges. In our study the edges, terms of puddle depth, were the thinnest part of a puddle.

\subsubsection{IR model - determination of a}

The relative difference $(R D$, Eq. (5.4)) was lowest at $a=0.76$, with mean $R D=$ $<0.01$ ( $\mathrm{SD}=0.15)$. This value for $a$ was used in the regression analysis.

Table 5.2 shows the multiple linear regression analysis results (Eq. (5.5)). The $\mathrm{R}^{2}$ adj was 0.95 . The coefficients $b_{0}$ and $b_{2}$ were not statistically significantly different from zero. The coefficient $b_{1}$ was statistically significantly different from zero, which represented the linear relation between $A_{p, I R}$ and $A_{p, G T}$. By executing the linear regression again, without the terms $b_{0}$ and $b_{2}$, the remaining linear term $b_{1}$ was 1.0253 (with SE 0.011), which is close to 1 . The $\mathrm{R}^{2}$ adj was 0.95 and the Root-Mean-SquareError (RMSE) was 0.0332 resulting in a $95 \%$ confidence interval of $0.0332 \cdot 1.96=$ $0.0651 \mathrm{~m}^{2}$. With the succeeding model $y=1.0253 * x$, the F-test resulted in a F-value 
Table 5.2: Multiple linear regression results (Eq. (5.5)), with the coefficients, the estimates (SE) and the p-value.

\begin{tabular}{crr}
\hline coefficient & estimate $(\mathrm{SE})$ & $\mathrm{p}$-value \\
\hline$b_{0}$ & $-0.029(0.025)$ & 0.251 \\
$b_{1}$ & $1.028(0.026)$ & $<0.001$ \\
$b_{2}$ & $0.002(0.002)$ & 0.282 \\
\hline
\end{tabular}

of 8000.9 with p-value $<0.001$. So, $b_{1}$ was statistically significant different from 1 and $A_{g, I R}(x)$ was therefore slightly underestimating the puddle area compared to $A_{p, G T}(y)$ for larger puddles. This was visualised by Fig. 5.4 which shows the scatter plot of $A_{g, G T}(y)$ against $A_{g, I R}(x)$, including the linear regression line and the $95 \%$ prediction intervals.

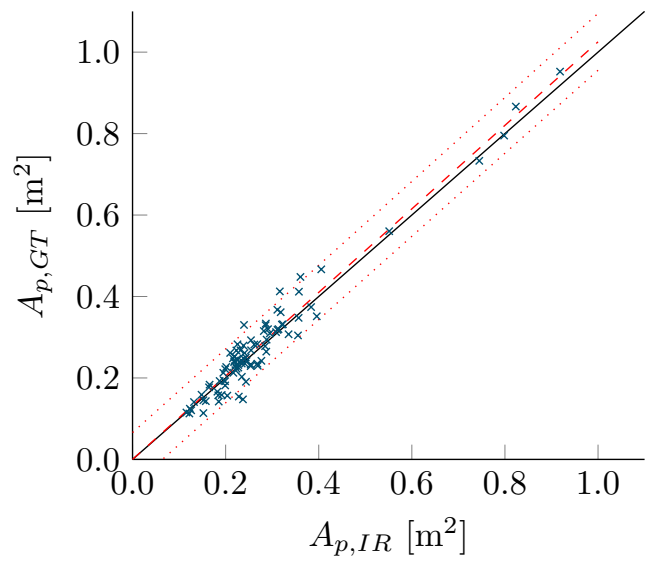

Figure 5.4: Scatter plot of $A_{g, G T}$ against $A_{g, I R}$ with (-) identity line, (---) fit: $y=1.0253 * x,(\cdots \cdots) 95 \%$ prediction intervals.

\subsection{Discussion}

\subsubsection{Measurement method}

The working principle of the IR method is based on temperature and emissivity differences. First, the emissivity of water was 0.96 , and for concrete 0.95 (dry) or 0.97 (rough), which is about equal (FLIR, 2011). Second, it should be noted that a fresh urine puddle quickly cools to the floor temperature, especially at the edges. Within 10 min a puddle cools to the floor temperature inside a cow house (Snoek et al., 
2014a). Therefore, to obtain IR images of complete puddles image collection has to be done as soon as a cow has finished urinating. In this study the time between puddle application and image collection was close to $1 \mathrm{~s}$ but no more than 5 seconds. This timing did not affect the estimated puddle area.

\subsubsection{Image calibration}

It was not possible to automatically calibrate images to determine real-world pixel values for three reasons: (1) the floor's design was often hardly visible in the IR or the colour image, (2) the design dimensions varied, and (3) no object with known dimensions could be found that was clearly visible in both the IR and colour images and easy to use for automatic calibration. However, this procedure did not need to be automated as the total number of puddle images was limited and the camera was manually operated. With the applied method, each time the trolley was built up a new image of the same aluminium plate was taken for calibration. The aluminium plate was never at the same location in the camera's field of view, but it was always located more or less in the centre, similar to the puddles. Moreover, the aluminium plate was always clearly distinguishable from its surrounding because of the colour and the light reflection, and it was possible to zoom in the plate during pixel selection. Therefore, the possible incorrect pixel selection was expected to be not more than 1 pixel per corner. Additionally, the total real-world area in an image was about $2.0 \mathrm{~m}^{2}$, covered by $480 \cdot 640=307200$ pixels. These dimensions resulted in an area of only $6.5 \times 10^{-6} \mathrm{~m}^{2}$ per pixel. As such, a 1 pixel error was negligibly small. We did not calibrate the temperature values measured by the IR camera since we did not need these for our purpose. In conclusion, the calibration method performed well with a negligibly small error.

\subsubsection{Determine puddle area}

The Ground Truth puddle area $\left(A_{p, G T}\right)$ was the averaged area estimated by three annotators. The results showed that the annotators agreed well with each other with a mean Coefficient of Variation (CV) of 0.04 (Fig. 5.2).

The IR puddle area $\left(A_{p, I R}\right)$ was determined with the minimum threshold $\left(t h l d_{\min }\right)$ algorithm (Eqs. (5.1) to (5.3)). The assessment of agreement between $A_{p, I R}(a=$ 0.76 ) and $A_{p, G T}$ showed a good fit (Fig. 5.4 and Table 5.2). Even though the linear model was statistically significantly different from the identity line $y=x$; the linear regression had an interception with the origin, the systematic underestimation was only $1.0253-1=0.0253 \mathrm{~m}^{2}$ per $1 \mathrm{~m}^{2}$ puddle size increase, and the noise was only $0.0651 \mathrm{~m}^{2}$. In addition, the $\overline{T_{\text {background }}}$ had no effect on the puddle area estimation. Therefore, to correct the $A_{p, I R}$ of future puddle measurements, the regression model (Eq. (5.6)) can be used as calibration. With an expected urine puddle area of $0.2 \mathrm{~m}^{2}$ to 
$1.2 \mathrm{~m}^{2}$, and taking into account the ground truth accuracy and the related regression model Eq. (5.6), the measurement uncertainty of the IR camera method is $<0.1 \mathrm{~m}^{2}$.

$$
A_{p, G T}=1.0253 \cdot A_{p, I R}
$$

\subsection{Conclusions}

The objective of this study was to improve and to validate the IR camera method to determine the area of a warm fluid layer. The ground truth reference had a mean Coefficient of Variation of 0.04 among the three annotators. The developed IR threshold algorithm was able to automatically select the puddle from an IR-image based on its temperature values. The related estimated puddle area was almost equal to the ground truth, with a linear regression model (Eq. (5.6)) to calibrate the estimated area of future puddles. In addition, the $\overline{T_{\text {background }}}$ had no effect on the puddle area estimation. These results were realised by using an IR-image that was obtained within $5 \mathrm{~s}$ after puddle application.

With an expected urine puddle area of $0.2 \mathrm{~m}^{2}$ to $1.2 \mathrm{~m}^{2}$, and taking into account the ground truth accuracy and the related regression model, the measurement uncertainty of the IR camera method is $<0.1 \mathrm{~m}^{2}$.

Future perspectives for the use of the developed IR method in commercial dairy cow houses. As described in the introduction, the usage of the IR camera mounted on a trolley in a cow houses was successfully tested already. Before entering the cow area, an IR image of the aluminium plate on the floor will be taken for calibration purpose. Among the cows one will wait before a cow urinates. In case a fresh puddle is nearby one can go there as quick as possible and take images of this puddle. Based on the preliminary research, we expect that it is possible to be in time, before a puddle starts to cool down already. Only puddles that can be reached in time will be measured. The developed method is able to distinguish a puddle from its surroundings, irrespective of the presence of faeces and dirt.

\subsection{Acknowledgements}

The authors would like to thank M. Kluivers-Poodt for facilitating the use of the IR-camera. Language editing of a near-final draft of the paper was provided by P. Griffith. 


\section{CHAPTER 6}

\section{Assessing fresh urine puddles in commercial dairy cow houses part 1: surface area and depth}

Dennis Snoek

Hans Stigter

Sam Blaauw

Peter Groot Koerkamp

Nico Ogink

Submitted for Publication 


\begin{abstract}
To lower ammonia emissions, the EU has set national emission ceilings. In The Netherlands, new emission reduction methods for dairy cow houses are pre-assessed by an emission model. However, the model's required inputs have not been updated since 2000. Moreover, little is known about how the approved and widely applied reduction methods, floor design and manure scraper application, affect the model's input variables for puddle area $\left(A_{p}\right)$ and depth $\left(D_{p}\right)$. Therefore, the objective of this paper is to assess the $A_{p}$ and $D_{p}$ of fresh urine puddles in commercial dairy cow houses. Sixteen commercial farms were measured in a factorial design of four Floor-Management types (FMTypes). Each farm was measured in two seasons and underwent an intense-floor-cleaning treatment (PREclean) before puddle creation for the $D_{p}$ measurement, which was compared with those created under normal floor conditions with on-farm manure scraping. Overall mean values were $0.83 \mathrm{~m}^{2}$ for $A_{p}$ and $1.0 \mathrm{~mm}$ for $D_{p}$. For both $A_{p}$ and $D_{p}$ the variation within a farm was large but negligible between farms. FMType significantly affected both variables. The V-shaped asphalt floor resulted in larger $A_{p}\left(1.04 \mathrm{~m}^{2}\right)$ and $D_{p}(1.5 \mathrm{~mm})$ values than did the slatted and grooved floors $\left(0.76 \mathrm{~m}^{2}, 0.93 \mathrm{~mm}\right)$. Compared to the reference values, the $A_{p}$ values were similar, but the $D_{p}$ values and variation were 3 to 6 times larger. Finally, PREclean resulted in $D_{p}$ values that were 3 times lower than those with on-farm scraping. In short, good floor cleaning has the potential to strongly reduce ammonia emission.
\end{abstract}




\subsection{Introduction}

Ammonia $\left(\mathrm{NH}_{3}\right)$ emission strongly contributes to the acidification and eutrophication of the environment. To restrict $\mathrm{NH}_{3}$ emission, the EU has set National Emission Ceilings (NEC) (EU, 2001) that, in the last 25 years, have substantially decreased $\mathrm{NH}_{3}$ emission in the EU overall and in The Netherlands specifically (EEA, 2012). Nevertheless, the latest (2012) reported total $\mathrm{NH}_{3}$ emission in The Netherlands (136 kt) is still above the current $\mathrm{NEC}$ (128 kt) (EEA, 2015). In general, $\mathrm{NH}_{3}$ emission in the EU is dominated by agriculture, which accounts for almost $95 \%$ of the total emissions in the EU-27 (EEA, 2015). Within agriculture, dairy cow houses represent one of the largest sources of $\mathrm{NH}_{3}$ and their contribution in The Netherlands is estimated to be about $13 \%$ (Velthof et al., 2012). In a cow house, $\mathrm{NH}_{3}$ emission originates from urine puddles on the floor and from slurry in the pit, which is generally underneath the whole cow house in The Netherlands. For a typical Dutch cow house with a concrete slatted floor, the floor-pit emission ratio is estimated to be about $70: 30 \%$ (Monteny et al., 1998).

To reduce the $\mathrm{NH}_{3}$ emission from dairy cow houses, farmers have to apply approved $\mathrm{NH}_{3}$ emission mitigation methods in new dairy cow houses. The available methods are listed in the 'Regulation on ammonia from livestock production', coded Rav (Tac-Rav, 2016), and new methods are regularly being added to the list. However, before a method becomes part of the Rav-list, it is assessed by a governmental Technical Advisory Committee (Tac-Rav). Experts in the Tac-Rav use an $\mathrm{NH}_{3}$ emission model to pre-assess new low-emission cow houses by estimating to what extent the applied mitigation method as well as the floor and management characteristics affect the floor and slurry pit emission. Based on this pre-assessment, these new methods are assigned provisional emission factors that are later replaced by definite emission factors after $\mathrm{NH}_{3}$ emissions have been measured in practice according to a protocol, which is described in Ogink et al. (2013). The model used in the pre-assessment phase was developed by Monteny et al. (1998) for research purposes and it requires estimates of several variables that are representative for practical conditions in a cow house such as a puddle surface area, $\mathrm{pH}$, and urea concentration. However, little information is known about these practical conditions, and as such, it is difficult to accurately estimate input values that reflect floor and management characteristics of new housing designs. This scarcity of information is mainly caused by the complexity of measuring required floor and manure storage parameters under real cow house conditions. What little information we have on the model variables is mostly based on research done before 2000 under cow house and management conditions that may have changed since then, for example the floor design. For the pre-assessment process, there is a need both for a simpler, more pragmatic emission model, i. e. a model with fewer input variables, and for actual measurement data of these input parameters from dairy cow 


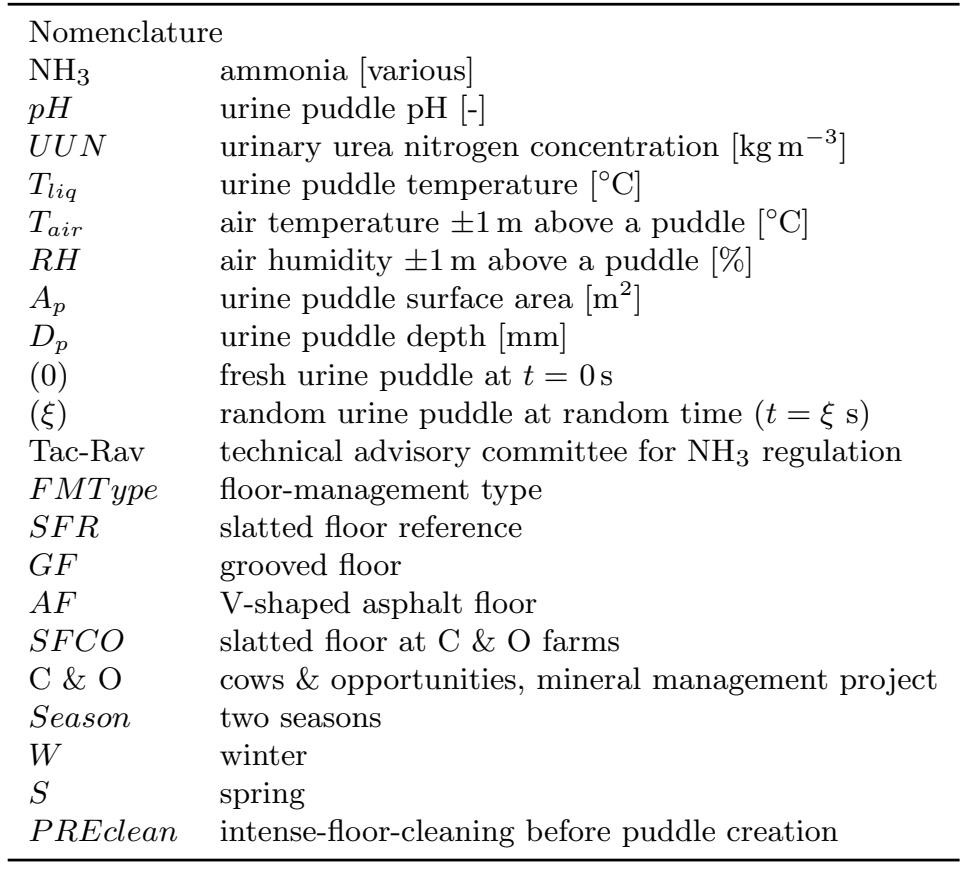

houses reflecting current practical conditions.

To simplify the Monteny model and to identify its most important variables, the model was tested in a sensitivity analysis along with comparable models from other studies (Snoek et al., 2014b). The analysis showed that five puddle-related input variables: $\mathrm{pH}$, depth, area, urea concentration, and temperature, were the most important ones to explain variation in $\mathrm{NH}_{3}$ emission from puddles on the floor. These findings hold true regardless of the model parameters. The remaining four variables were the air temperature and air velocity just above a puddle, the maximum rate of urea conversion or urease activity, and the Michaelis-Menten constant. The study also concluded that hardly any measurement data is available on these variables from urine puddles in commercial dairy cow houses. In other words, values, distributions, and correlations of the variables were unknown. Without accurate data, it is not possible to develop an accurate $\mathrm{NH}_{3}$ emission model or to calibrate existing models.

To obtain this data, fresh dairy cow urine puddles were measured in a variety of cow house designs based on the floor type in the cow walking area. New types of floors are one of the main approved and applied $\mathrm{NH}_{3}$ emission reduction methods in The Netherlands, together with the use of a manure scraper (Tac-Rav, 2016). Both floor design and a manure scraper affect the physical characteristics of urine and faeces and thus affect $\mathrm{NH}_{3}$ emission (Braam et al., 1997a,b; Poteko et al., 2014). The extent of these effects, however, is limitedly known (Braam et al., 1997a) because the area $\left(A_{p}\right)$ 
and depth $\left(D_{p}\right)$ values of puddles, as described in the Monteny model, are not part of the Tac-Rav measurement protocol (Ogink et al., 2013) used to assess the emission of newly built housing systems. Consequently, no information is available on these variables. In this paper, therefore, we focused on the floor design and scraping in relation to the physical characteristics of a urine puddle.

The results from our field study are reported in two papers. The results of the chemical characteristics of a urine puddle: the $\mathrm{pH}$ and urea concentration, plus temperature, are described in Snoek et al. (2016d). The objective of the current paper is to assess the puddle area $\left(A_{p}\right)$, puddle depth $\left(D_{p}\right)$, and the related puddle volume $\left(V_{p}\right)$ in commercial dairy cow houses and the effect of floor type, season and intense manure scraping on these variables.

\subsection{Materials and Methods}

\subsubsection{Experimental design}

\section{Design and experimental factors}

The design of a typical dairy cow house in the Netherlands, hereafter called the reference situation, consists of a living area where cows can walk around on a concrete slatted floor, rest in cubicles, and eat at a feeding alley. The slatted floor area is about $3.5 \mathrm{~m}^{2} \mathrm{cow}^{-1}$. A slurry pit is located beneath the slatted floor, the cubicles and the feeding alley. The experimental design had a factorial set up with four types of farms based on floor design and farm management, labelled FMType, and each FMtype contained four farms. FMType 1 farms had a slatted floor that was equal to the reference situation $(S F R)$. Cow house layout and farm management were diverse among the four farmers. FMType 2 farms had a completely closed, grooved floor $(G F)$. The layout and the farm management was similar for all cow houses. FMType 3 farms had a completely closed, V-shaped asphalt floor $(A F)$. The layout of the cow houses was about similar, but farm management was diverse among the four farmers. FMType 4 farms had a slatted floor, like FMType 1, but these four farmers were involved in the Cows \& Opportunities project $(S F C O)$. In the Cows \& Opportunities project the farmers actively manage several factors, including the nutrient cycle at their farm to lower $\mathrm{NH}_{3}$ emission (Oenema et al., 2001; Aarts et al., 2015). The layout of the cow houses was diverse among the four SFCO farmers. Figure 6.1 show examples of all FMTypes.

In total, we measured 344 fresh dairy cow urine puddles at 16 commercial dairy farms, spread over The Netherlands. Measurements were performed in two seasons, represented by the experimental factor Season. Each farm was measured both in winter $(W)$ and in spring $(S)$, and farms were measured in random order per season. 
The winter period covered November 2014 to January 2015. The spring period ran from the end of March 2015 to the beginning of June 2015. Each individual farm measurement was performed on a single day and started in the morning between nine and ten o'clock. Cows were inside the cow houses at all farms before and during the measurements.

A manually operated hand manure scraper was used for the puddle depth $\left(D_{p}\right)$ measurement (Fig. 6.3). The $D_{p}$ was first measured in floor puddles in the "as-is" situation. This situation reflects the floor conditions under normal on-farm conditions, including a manure scraper cleaning the floor if present. The $D_{p}$ of floor puddles was then measured where the floor had been pre-cleaned with a hand scraper before the puddle was created. With the manually operated hand scraper, we investigated the potential influence of intense floor cleaning on $D_{p}$. This experimental factor was called PREclean and was applied at each farm.

\section{Dairy farm characteristics}

Fourteen of the 16 dairy cow houses were recently built and had an average age of about 5 yrs. For the remaining two houses, both $S F C O$ farms, the older part of the farms (about 27 yrs old) was expanded with newly built parts. These new parts had an average age of about $6 \mathrm{yrs}$. In these two houses, measurements took place in both the old and the new part because these areas formed one connected area for the dairy cows. Eleven farms had an automatic milking system (AMS), four farms had a conventional side-by-side milking parlour, and one had a rotary milking parlour. The farms with an AMS milked the cows 2.8 times a day, on average, and the other farms milked the cows twice a day.

Figure 6.1 shows examples of manure scrapers and their cleaning effect. Eight farms had a conventional, pulled manure scraper that scraped the whole cow house floor, except the cross-paths, 2 to 12 times a day. Six farms used a manure scraper robot that ran continuously at each farm. However, in all cases the robots needed to load their batteries several hours a day and mainly at night. In addition, the robots' driving settings varied among the farms. There were two farms without a manure scraper. They both irregularly scraped the floor with either a compact wheel loader or with a hand scraper. The hand scraper was similar to the one used for the applied PREclean treatment (Section 6.2.1 and Fig. 6.3). A hand scraper was used by all farmers to scrape the cubicle bedding, cubicle sides, and sometimes parts of the floor. The type of cubicle bedding material varied among the farms. The most used bedding was a soft mattress or a water mattress, combined with a litter consisting of sawdust (10 farms). The remaining six farms used a deep litter cubicle, filled with either sand (1 farm), straw (2 farms), or dried faeces processed from their own slurry (3 farms). 


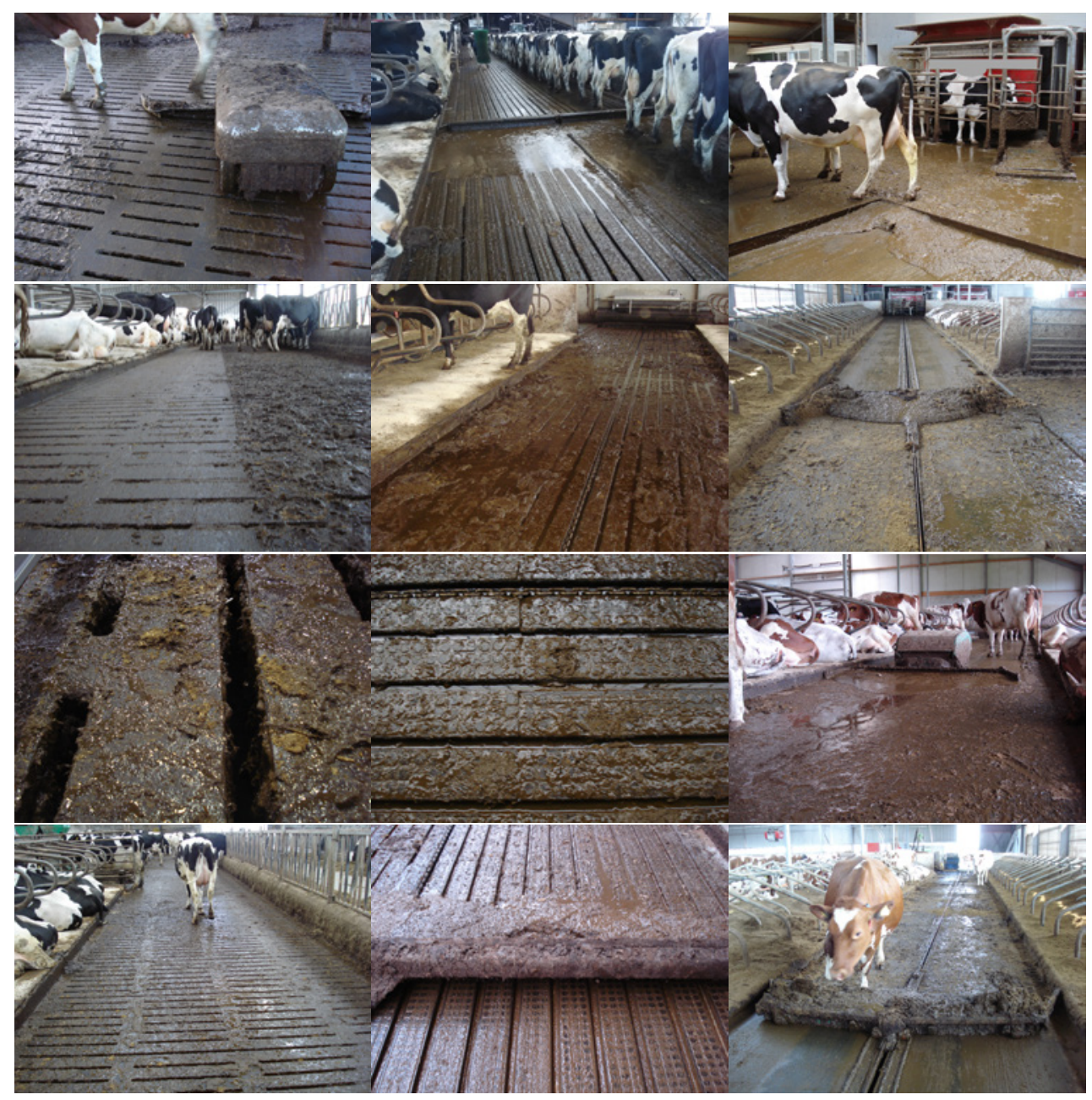

Figure 6.1: Floor design and effect of manure scrapers. Left column: slatted floor $(S F)$, middle column: grooved floor $(G F)$, right column: V-shaped asphalt floor $(A F)$.

\subsubsection{Measurements}

\section{Measured variables}

Figure 6.2 shows the equipment used on the floor inside a dairy cow house. At each farm we first measured 10 fresh urine puddles immediately after the cow had urinated $(t=0 \mathrm{~s})$. Of each fresh puddle we measured the puddle area $\left(A_{p}(0)\right)$. We also measured the $p H(0)$, the urinary urea nitrogen concentration $(U U N(0))$ and the puddle temperature $\left(T_{l i q}(0)\right)$ of these puddles, and the local air temperature $\left(T_{\text {air }}(0)\right)$, 
and relative humidity $(R H(0))$ just above them, which are all five described in Snoek et al. (2016d). After measuring 10 fresh puddles, we measured 10 randomly selected urine puddles that were already present on the floor for a random time $(t=\xi \mathrm{s})$. We measured the $p H(\xi)$ and the puddle temperature $\left(T_{l i q}(\xi)\right)$ of these puddles, and the local air temperature $\left(T_{\text {air }}(\xi)\right)$, and relative humidity $(R H(\xi))$ just above them, which again are described in Snoek et al. (2016d). Finally, we measured the puddle depth $\left(D_{p}(0)\right)$ of 63 puddles manually created with clean fresh urine collected at the same farm.

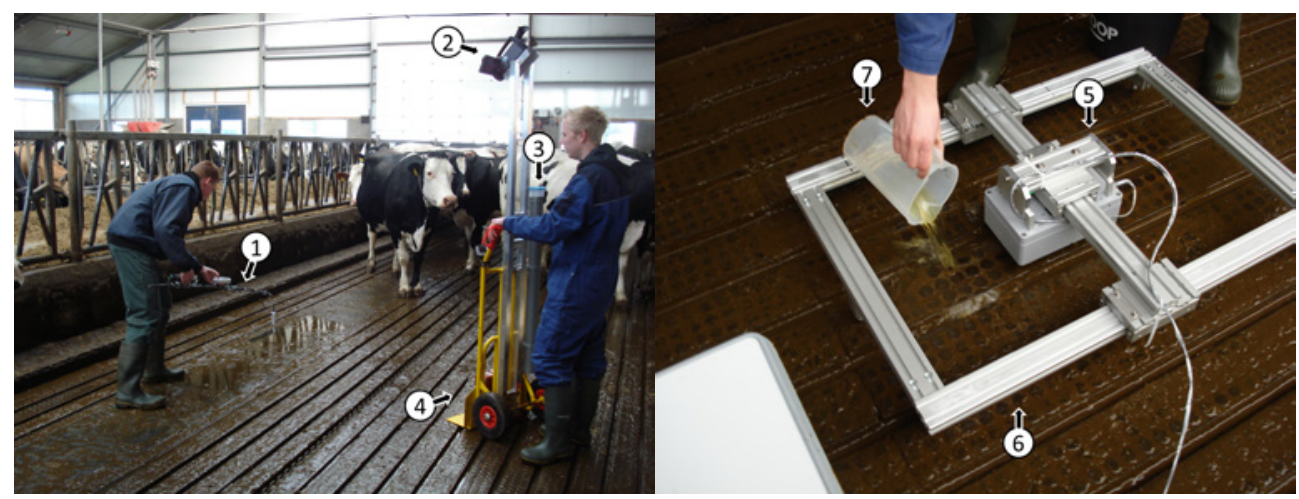

Figure 6.2: Measurement setup with on the left (1) the sensor rod for $p H, T_{\text {liq }}, T_{\text {air }}$ \& $R H$, (2) the IR camera for $A_{p}$, (3) the urine sample holder, (4) the moveable trolley, and on the right (5) the ultrasonic device for $D_{p},(6)$ the XY-table, and (7) the measuring jug to create a puddle.

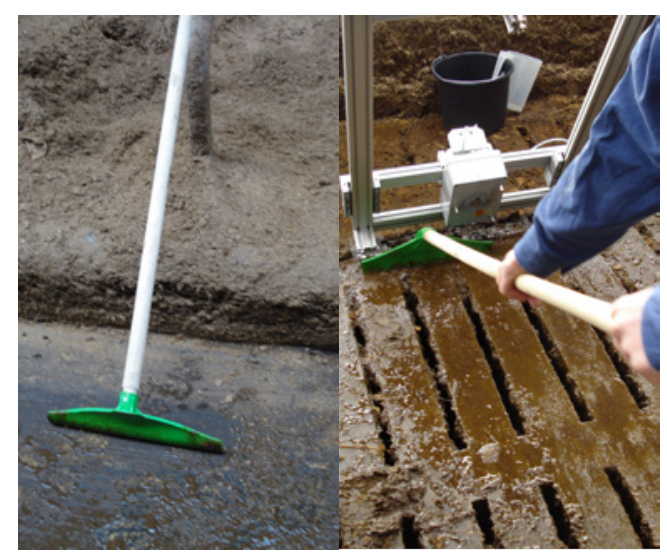

Figure 6.3: The hand scraper (left) and the use on a slatted floor (right). 


\section{Equipment}

To measure $A_{p}$ we used a custom made camera-trolley (Fig. 6.2), as described in Snoek et al. (2016a). On top of the trolley, a thermal infrared (IR) camera was mounted (FLIR SC660; FLIR Systems, Inc. USA) that was able to simultaneously take an IR and a colour image. Images were recorded automatically every $20 \mathrm{~s}$. First, an IR image of a puddle without sensors or other obstructions was taken. This image was then used in the developed IR model (Snoek et al., 2016a). The remaining IR images contained parts of sensors or other obstructions that were used for additional information about the measurement circumstances. Using the IR image and the IR model, the $A_{p}(0)$ was determined with an accuracy of $0.1 \mathrm{~m}^{2}$. More details of the IR camera and IR model can be found in (Snoek et al., 2016a). The camera-trolley was also used to transport jars for the collection of urine samples to determine $U U N(0)$ in the lab (Snoek et al., 2016d). To carry the jars, a piece of circular rainwater pipe with caps was mounted to the trolley.

To measure $D_{p}$, we used a custom made XY-table with an ultrasonic distance sensor (Fig. 6.2), as described in Snoek et al. (2015). The ultrasonic sensor (WLG2000, Inspection Technology Europe BV, NL) was operated by a laptop. A time series measurement was run with a $1 \mathrm{~s}$ time step and point measurements were simultaneously obtained by pressing the enter key on the laptop's keyboard. First, the distance to the floor under the present circumstances ("dry") was measured followed by the distance after a puddle was created ("wet"). The $D_{p}(0)$ was then calculated by subtracting the "wet" distance from the "dry" distance.

\section{Measurement protocol}

The whole measurement procedure was determined in a preliminary test series inside a commercial dairy cow house. The variables $A_{p}(0), p H(0), U U N(0), T_{l i q}(0), T_{\text {air }}(0)$, and $R H(0)$ were measured at the same time on the same fresh puddle, according to the fresh-puddle procedure. After measuring 10 fresh puddles, one person continued to measure 10 randomly selected urine puddles that were already on the floor $(t=\xi \mathrm{s})$. The $p H(\xi), T_{l i q}(\xi), T_{a i r}(\xi)$, and $R H(\xi)$ were measured at the same time on the same random puddle, according to the random-puddle procedure. Based on the preliminary tests, we concluded that it was impossible to measure the $D_{p}(0)$ of these fresh urine puddles. Therefore, we decided to determine $D_{p}(0)$ in separate manually-created urine puddles, according to the $D_{p}$-procedure. The following three procedures were used in the measurement protocol:

The fresh-puddle procedure (Fig. 6.2):

1. Each puddle measurement was started $1 \mathrm{~s}$ to $15 \mathrm{~s}$ after a cow had finished urinating; 
2. Person 1 gently cleared the way to approach the puddle and kept the cows at a distance;

3. Person 2 started the IR image recording of the puddle for $60 \mathrm{~s}$ at a $20 \mathrm{~s}$ interval to determine $A_{p}$;

4. After collecting the first image, person 3 placed the sensor rod in the urine puddle to measure the $p H(0), T_{l i q}(0), T_{a i r}(0)$, and $R H(0)$ for $60 \mathrm{~s}$ with $2 \mathrm{~s}$ interval;

5. After starting the IR image recording, person 2 placed a filter in the puddle to collect a urine sample to determine $U U N(0)$. When the filter was completely soaked with urine, it was put in a jar with an acid to immediately stop the urea conversion process;

6. Person 1 made notes, took photos and protected the measurement area;

The random-puddle procedure:

1. Person 3 randomly selected a puddle;

2. Person 3 placed the sensor rod in the puddle to measure the $p H(\xi), T_{l i q}(\xi)$, $T_{\text {air }}(\xi)$, and $R H(\xi)$ for $20 \mathrm{~s}$ with $2 \mathrm{~s}$ interval;

The $D_{p}$-procedure (Fig. 6.2):

1. All three persons collected fresh urine from the cows by using buckets;

2. The XY-table was placed on the floor at a random location;

3. The distance to the floor under the present circumstances ("dry") was measured one-by-one at 9 points within the XY-bounds (Snoek et al., 2015);

4. A puddle was made by pouring 0.21 of the collected urine with a measuring jug at point 1 from a height of about $0.1 \mathrm{~m}$;

5. The distance to the created urine puddle ("wet") was measured;

6. Steps 4 and 5 were repeated until all 9 points were measured;

7. Steps 2, 3, 4 and 5 were repeated 4 times:

(a) In total 5 unique, randomly selected locations were measured;

(b) At 2 measurement locations, steps 3, 4 and 5 were repeated, but before step 3 was done, the floor was manually cleaned (PREclean; Fig. 6.3). In total, 45 puddles were measured under the present circumstances without PREclean treatment, and 18 puddles with the PREclean treatment.

\section{Equipment calibration}

To calibrate the collected IR images of the urine puddles, we took both an IR and a colour image of a rectangle aluminium plate of $1.0 \mathrm{~m} \cdot 0.6 \mathrm{~m}$, as described in Snoek et al. 
(2016a). These plate images were collected on-site at the start of each measurement day after the camera-trolley was built up. The IR image was used in the point coordinates calibration method. The temperature values in the IR image were roughly correct, but they were not calibrated since we did not need the absolutely correct temperature values in the images.

To calibrate the distance measurements by the ultrasonic device, we applied the balance method as described in Snoek et al. (2015) in experiment 1. This was done twice: before the winter measurement series and before the spring measurement series.

The calibration of the sensor rod is described in Snoek et al. (2016d).

\subsubsection{Data analyses}

\section{Incorrect values}

Parts of cow legs were occasionally present in the IR images. This resulted in an incorrect $A_{p}(0)$ value. Because of this, $11 \mathrm{IR}$ images and their related $A_{p}(0)$ values were excluded.

\section{Calculation of puddle volume}

With the variables $A_{p}(0)\left[\mathrm{m}^{2}\right]$ and $D_{p}(0)[\mathrm{mm}]$ the puddle volume $V_{p}(0)\left[\mathrm{m}^{3}\right]$ was calculated according to Eq. (6.1).

$$
\overline{V_{p}(0)}=\overline{A_{p}(0)} \cdot \overline{D_{p}(0)} \cdot 10^{-3}
$$

Since the $A_{p}(0)$ and $D_{p}(0)$ were not measured at the same time in the same puddle, we used the mean values per farm and per Season for both variables. For $D_{p}(0)$ we also distinguished between the data with and without PREclean treatment. This resulted in a total of $16 \cdot 2 \cdot 2=64 \mathrm{NH}_{3} S(0)$ values, where two values were missing because the PREclean treatment was not applied in Season $W$ at farms $F 1$ and $F 6$.

\section{Statistical analyses}

We performed a restricted maximum likelihood method (REML) (Payne et al., 2015) to study group effects. The models used are given in Eq. (6.2) for $A_{p}(0)$, in Eq. (6.3) for $D_{p}(0)$ and in Eq. (6.4) for $V_{p}(0)$.

$$
\begin{aligned}
A_{p, i j k}(0) & =\mu+T y_{p}+S e_{r}+F a_{i}+S e_{i j}+\epsilon_{i j k} \\
D_{p, i j l m}(0) & =\mu+T y_{p}+C l_{q}+S e_{r}+F a_{i}+S e_{i j}+L o_{i j l}+\epsilon_{i j l m} \\
V_{p, i j n}(0) & =\mu+T y_{p}+C l_{q}+S e_{r}+F a_{i}+S e_{i j}+\epsilon_{i j n}
\end{aligned}
$$


With the fixed part of the model:

- $\mu$ the overall constant (grand mean),

- $T y_{p}$ the main effect of FMType (with $p$ the FMType assigned to ijklmn),

- $C l_{q}$ the main effect of PREclean (with $q$ the PREclean assigned to ijklmn)

- $S e_{r}$ the main effect of Season (with $r$ the Season assigned to ijklmn),

The random part of the model:

- $F a_{i}$ the effect of Farm $i(i=1 . .16)$,

- $S e_{i j}$ the effect of Season $j$ ( $j=$ winter, spring) within Farm $i$,

- $L o_{i j l}$ the effect of measurement-Location $l(l=1 . .5)$ within Farm $i$ and Season $j$,

- $\epsilon_{i j . .}$ the residual error:

- for unit $i j k$ in Eq. (6.2), representing the Puddle $k(k=1 . .10)$ within Farm $i$ and Season $j$.

- for unit $i j l m$ in Eq. (6.3), representing the measurement-Point $m(m=1 . .9)$ within Farm $i$, Season $j$ and Location $l$.

- for unit $i j n$ in Eq. (6.4), representing the variance $n(n=-)$ within Farm $i$ and Season $j$.

The random terms $L o_{i j l}$ and $\epsilon_{i j l m}$ were, respectively, the location of the XY-table at the floor within a farm and a season, and the measurement point of the ultrasonic sensor within the XY-table, thus within $L o_{i j l}$.

We estimated the variance components of each random term, the related coefficient of variation $(\mathrm{CV})$, and the Wald-statistic for the fixed terms. The variance component measures the variability of a term over and above the variability of the sub-units of which it is composed (Payne et al., 2015). The CV is the square root of the variance component divided by the mean response $(\mu)$ times 100 .

The hypothesis $\left(h_{0}\right)$ was tested for all three variables. The values of a variable from hypothesis $\left(h_{0}\right)$ were from populations with the same mean per group compared to the alternative hypothesis $\left(h_{1}\right)$ where the population means were not the same per group. The predicted means were computed, and if $h_{0}$ was rejected, the approximate least significant differences (LSDs) were computed for these predicted means of the fixed terms to identify the statistical significant differences between subgroups. All tests were calculated by using a significance level of $\alpha=0.05$.

Finally, a scatter plot was made of the $\overline{A_{p}(0)}$ against the $\overline{D_{p}(0)}$ as mean per Farm and per Season. A linear regression was carried out between these two variables. 


\subsection{Results}

\subsubsection{Statistical analyses}

Tables 6.1 to 6.3 show the results of the REML analysis. Table 6.1 shows that for $A_{p}$ the between-Farm variance of $A_{p}(0)$ was limited $(\mathrm{CV}=4.4 \%)$ compared to both the within-Farm variance $(\mathrm{CV}=12.2 \%)$ and especially the variance at puddle level within Season $(\mathrm{CV}=29.2 \%)$. For $A_{p}(0)$ the fixed term FMType showed a statistically significant effect $(\mathrm{p}=0.002)$. The effect of Season was not statistically significant ( $\mathrm{p}>0.05)$. Therefore, we reduced the model from Eq. (6.2) to Eq. (6.5) by dropping the term:

$$
A_{p, i j k}(0)=\mu+T y_{q}+F a_{i}+S e_{i j}+\epsilon_{i j k}
$$

with symbols according to Eq. (6.2) (Section 6.2.3). Table 6.2 shows that the largest, statistically significant puddle areas occurred at FMType $=A F$, which is the Vshaped, solid asphalt floor. The predicted mean $A_{p}(0)$ for this floor type was $1.04 \mathrm{~m}^{2}$. The other FMTypes ( $S F R, G F$, and $S F C O$ ) had predicted mean $A_{p}(0)$ values of $0.72 \mathrm{~m}^{2}$ to $0.80 \mathrm{~m}^{2}$. These values were not statistically significantly different from each other.

Table 6.1: The Variance Component (Var) \& Coefficient of Variation (CV) for the defined random terms and the residual error $\epsilon$, followed by the F-statistic \& p-value for the fixed terms. For $A_{p}(0), D_{p}(0)$, and $V_{p}(0)$.

\begin{tabular}{|c|c|c|c|c|c|c|}
\hline \multirow[b]{2}{*}{ Term } & \multicolumn{2}{|c|}{$A_{p}(0)\left[\mathrm{m}^{2}\right]$} & \multicolumn{2}{|c|}{$D_{p}(0)[\mathrm{mm}]$} & \multicolumn{2}{|c|}{$V_{p}(0)^{\dagger}\left[\mathrm{m}^{3}\right]$} \\
\hline & $\begin{array}{r}\text { Var (s.e.) } \\
\cdot 10^{-2}\end{array}$ & $\mathrm{CV}$ & $\begin{array}{r}\text { Var (s.e.) } \\
\cdot 10^{-1}\end{array}$ & $\mathrm{CV}$ & $\begin{array}{r}\text { Var (s.e.) } \\
\cdot 10^{-8}\end{array}$ & $\mathrm{CV}$ \\
\hline$F a_{i}$ & $0.13(0.472)$ & 4.4 & $-0.16(0.259)$ & - & $-2.17(2.033)$ & - \\
\hline$S e_{i j}$ & $1.02(0.562)$ & 12.2 & $0.45(0.405)$ & 20.4 & $5.30(3.597)$ & 24.6 \\
\hline$L o_{i j l}$ & & - & $2.63(0.400)$ & 49.2 & & - \\
\hline \multirow[t]{2}{*}{ Residual $\epsilon$} & $5.81(0.474)$ & $29.2^{\# \mathrm{a}}$ & $6.84(0.228)$ & $79.4^{\# \mathrm{~b}}$ & $7.90(2.068)$ & $30.1^{\# \mathrm{c}}$ \\
\hline & F-stat & $\mathrm{p}-\mathrm{val}^{\ddagger}$ & F-stat & $\mathrm{p}-\mathrm{val}^{\ddagger}$ & F-stat & $\mathrm{p}-\mathrm{val}^{\ddagger}$ \\
\hline FMType & 9.34 & 0.002 & 6.51 & 0.008 & 15.75 & $<0.001$ \\
\hline PREclean & - & - & 322.33 & $<0.001$ & 84.09 & $<0.001$ \\
\hline Season & - & - & 8.96 & 0.009 & 8.41 & 0.011 \\
\hline
\end{tabular}

\rceil $V_{p}(0)$ was calculated according to Eq. (6.1).

The residual represent \#a Puddle, \#b Point, or \#c "within farm" variance.

$\ddagger$ p-value when dropping the individual term from the full fixed model.

Table 6.1 shows that for $D_{p}(0)$ the between-Farm variance was negligibly small (CV $=-)$ compared to the within-Farm variance $(\mathrm{CV}=20.4 \%$ to $79.4 \%)$. The variance caused by the XY-table locations and measurement points were especially large. For 
Table 6.2: The number of samples $(\mathrm{N})$ and the predicted means with s.e., per FloorManagement type (FMType), intense-floor-cleaning effect PREclean or Season effect, and the grand mean $\mu$. For $A_{p}(0), D_{p}(0)$, and $V_{p}(0)$.

\begin{tabular}{|c|c|c|c|c|c|c|}
\hline \multirow[b]{2}{*}{ Term } & \multicolumn{2}{|c|}{$A_{p}(0)\left[\mathrm{m}^{2}\right]$} & \multicolumn{2}{|c|}{$D_{p}(0)[\mathrm{mm}]$} & \multicolumn{2}{|r|}{$V_{p}(0)^{\dagger}\left[\mathrm{m}^{3}\right]$} \\
\hline & $N$ & Mean (s.e.) & $N$ & Mean (s.e.) & $N^{\ddagger}$ & $\begin{array}{l}\text { Mean (s.e.) } \\
\cdot 10^{-3}\end{array}$ \\
\hline$\mu$ & 333 & $0.83(0.024)$ & 1956 & $1.0(0.053)$ & 62 & $0.94(0.0422)$ \\
\hline $\begin{array}{l}\text {-FMType- } \\
\text { SFR }\end{array}$ & 90 & $0.72(0.048)^{\mathrm{a}}$ & 483 & $0.9(0.099)^{\mathrm{a}}$ & 15 & $0.69(0.0810)^{\mathrm{a}}$ \\
\hline$G F$ & 83 & $0.80(0.048)^{\mathrm{a}}$ & 504 & $0.8(0.113)^{\mathrm{a}}$ & 16 & $0.76(0.0931)^{\mathrm{a}}$ \\
\hline$A F$ & 83 & $1.04(0.048)^{b}$ & 492 & $1.5(0.103)^{\mathrm{b}}$ & 15 & $1.46(0.0842)^{\mathrm{b}}$ \\
\hline$S F C O$ & 77 & $0.75(0.049)^{\mathrm{a}}$ & 477 & $1.1(0.103)^{\mathrm{a}}$ & 16 & $0.85(0.0822)^{\mathrm{a}}$ \\
\hline $\begin{array}{l}\text {-PREclean- } \\
\text { off }\end{array}$ & - & - & 1381 & $1.5(0.052)^{\mathrm{b}}$ & 32 & $1.27(0.0541)^{\mathrm{b}}$ \\
\hline $\begin{array}{l}\text { on } \\
\text {-Season- }\end{array}$ & - & - & 575 & $0.7(0.064)^{\mathrm{a}}$ & 30 & $0.61(0.0567)^{\mathrm{a}}$ \\
\hline $\begin{array}{l}W \\
S\end{array}$ & $\begin{array}{l}- \\
-\end{array}$ & $\begin{array}{l}- \\
-\end{array}$ & $\begin{array}{r}944 \\
1012\end{array}$ & $\begin{array}{l}0.9(0.057)^{\mathrm{a}} \\
1.3(0.057)^{\mathrm{b}}\end{array}$ & $\begin{array}{l}30 \\
32\end{array}$ & $\begin{array}{l}0.78(0.0698)^{\mathrm{a}} \\
1.10(0.0677)^{\mathrm{b}}\end{array}$ \\
\hline
\end{tabular}

$\dagger V_{p}(0)$ was calculated according to Eq. (6.1) $\Rightarrow \ddagger \mathrm{N}$ is the number of Farm · Season $\cdot$ PREclean. a,b statistical significant subgroups based on the approximate LSD (0.05 level) of REML means.

Table 6.3: The number of samples $(\mathrm{N})$ and the predicted means with s.e. per FMType $\cdot$ PREclean, for $D_{p}(0)$ and $V_{p}(0)$. This interaction was not statistically significant. For the main effects, see Table 6.1.

\begin{tabular}{|c|c|c|c|c|c|}
\hline \multicolumn{2}{|c|}{ Group } & \multicolumn{2}{|c|}{$D_{p}(0)[\mathrm{mm}]$} & \multicolumn{2}{|r|}{$V_{p}(0)^{\dagger}\left[\mathrm{m}^{3}\right]$} \\
\hline FMType & PREclean & $N$ & Mean (s.e.) & $N$ & $\begin{array}{l}\text { Mean (s.e.) } \\
\cdot 10^{-3}\end{array}$ \\
\hline$S F R$ & $F$ & 348 & $1.4(0.091)$ & 8 & $1.02(0.0856)$ \\
\hline$S F R$ & $T$ & 135 & $0.5(0.100)$ & 7 & $0.36(0.0882)$ \\
\hline$G F$ & $F$ & 351 & $1.2(0.107)$ & 8 & $1.09(0.0976)$ \\
\hline$G F$ & $T$ & 153 & $0.3(0.114)$ & 8 & $0.43(0.0990)$ \\
\hline$A F$ & $F$ & 369 & $1.9(0.094)$ & 8 & $1.79(0.0891)$ \\
\hline$A F$ & $T$ & 125 & $1.0(0.103)$ & 7 & $1.13(0.0908)$ \\
\hline$S F C O$ & $F$ & 315 & $1.5(0.095)$ & 8 & $1.18(0.0877)$ \\
\hline$S F C O$ & $T$ & 162 & $0.6(0.101)$ & 8 & $0.52(0.0884)$ \\
\hline
\end{tabular}

$D_{p}(0)$ all fixed terms of Eq. (6.3) showed a statistical significant effect. Table 6.2 shows that the largest, statistically significant $D_{p}(0)$ values occurred at FMType $=A F$ 
$\left(D_{p}(0)=1.5 \mathrm{~mm}\right)$. This table also shows that intense-cleaning (PREclean) of the floor more or less halved the $D_{p}(0)$ from $1.5 \mathrm{~mm}$ to $0.7 \mathrm{~mm}$, and that the $D_{p}(0)$ was larger in the spring $(1.3 \mathrm{~mm})$ than in the winter $(0.9 \mathrm{~mm})$. Furthermore, as shown in Table 6.2 , the s.e. values for $D_{p}(0)$ are large compared to the predicted means. This result confirms the large within-Farm variance.

Table 6.1 shows that for $V_{p}(0)$ the between-Farm variance was a negligibly small value $(\mathrm{CV}=-)$ and that the within-Farm variance was in the same range as the variance for $A_{p}(\mathrm{CV}=24.6 \%$ to $30.1 \%)$. For $V_{p}(0)$ all fixed terms of Eq. (6.4) showed a statistically significant effect, as was also shown for $D_{p}(0)$. Table 6.2 shows that values for the $V_{p}(0)$ mainly follow the same pattern as the results for $D_{p}(0)$.

Table 6.3 shows the predicted means of the interaction between FMType and PREclean for both $D_{p}(0)$ and $V_{p}(0)$. This interaction was not statistically significant and was therefore not included in Eqs. (6.3) and (6.4). This table, however, clarifies in more detail the intense-floor-cleaning effect (PREclean) per FMType. Without PREclean treatment, the $D_{p}(0)$ varies from $1.2 \mathrm{~mm}$ to $1.9 \mathrm{~mm}$, and with PREclean treatment from $0.3 \mathrm{~mm}$ to $1.0 \mathrm{~mm}$. The $A F$ resulted in the largest peaks. For $V_{p}(0)$ the $A F$ with PREclean treatment $\left(1.13 \times 10^{-3} \mathrm{~m}^{3}\right)$ was as high as the other three floors without PREclean treatment $\left(1.02 \times 10^{-3} \mathrm{~m}^{3}, 1.09 \times 10^{-3} \mathrm{~m}^{3}\right.$ and $\left.1.18 \times 10^{-3} \mathrm{~m}^{3}\right)$.

\subsubsection{Relationships between measured variables}

Figure 6.4 shows the relation between the $\overline{A_{p}(0)}$ and $\overline{D_{p}(0)}$ as mean per Farm and per Season. The linear relation was not statistically significant and was therefore not included in Fig. 6.4. As shown in Fig. 6.4, the $\overline{A_{p}(0)}$ and $\overline{D_{p}(0)}$ are scattered and they do not depend on each other.

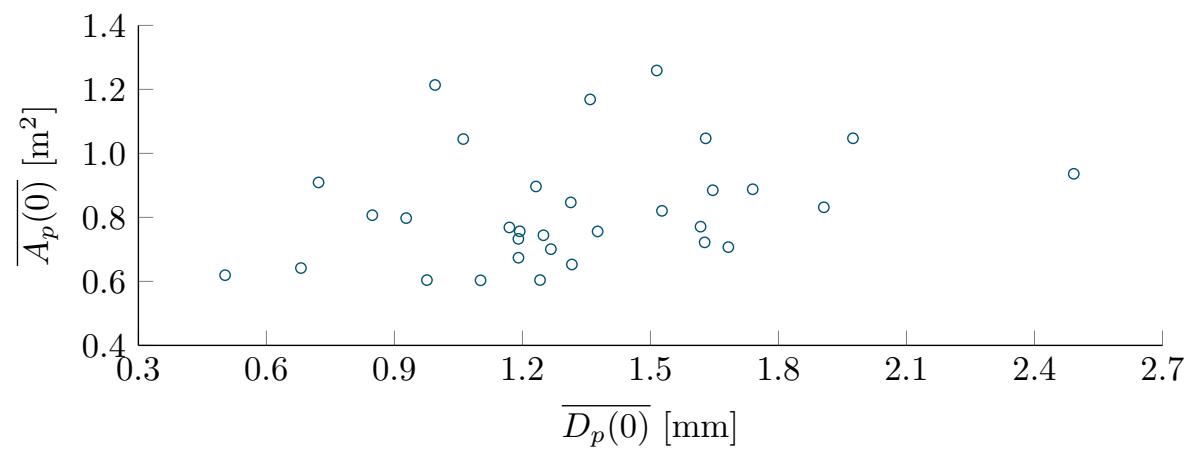

Figure 6.4: $\overline{A_{p}(0)}$ vs $\overline{D_{p}(0)}$ as mean per farm and per Season (०). 


\subsection{Discussion}

At the start of the current study, both measurement methods and information about urine puddle related variables from commercial dairy cow houses were hardly available. With the conducted measurements we gathered this unique information, which we can use to estimate the $\mathrm{NH}_{3}$ emission. Moreover, we can identify opportunities to improve the current measurement practice, as described in Ogink et al. (2013), and to reduce $\mathrm{NH}_{3}$ emission.

\subsubsection{Equipment and measurement performance}

Measuring the selected variables of urine on floors among dairy cows was rather complex. The test environment was unstructured and covered in manure, dirt and cows. During the measurements we had to proceed with caution and we focused on performing the measurements as accurately as possible. If a cow urinated far away from our "waiting position", we did not measure the puddle since we would be too late to obtain accurate readings and we would unnecessarily disturb the cows. The equipment was checked and calibrated both before and after each measurement day. The collected data was gathered, backed-up and verified directly after each measurement day. By doing so, we ascertained correct and accurate values.

We had 10 samples available per farm per season to analyse the $U U N$. In total, we sampled 16 farms $\cdot 2$ seasons $\cdot 10$ puddles $=320$ puddles. Occasionally, we measured some additional puddles with both the IR camera and the sensor rod, resulting in more measured puddles in total. However, we also had several missing values for all variables. These were caused by the presence of cows, sensor failure, or the final fine tuning of the measurement procedures at the first measured farms.

Finally, measured values for both $A_{p}$ and $D_{p}$ are biased. As discussed in Sections 6.4.2 and 6.4.3, they may underestimate the real values.

\subsubsection{Puddle area}

To estimate $A_{p}$, we observed that fresh urine had occasionally flowed outside the Field of View (FOV) of the IR camera for the Floor-Management types (FMTypes) GF and $A F$. The grooves of the grooved floor $(G F)$ transport fresh urine over the full length of the cow house, and this starts immediately during a urination. This resulted in a large area covered by urine within these grooves over a long distance, where it also mixed with urine that was already present. The slope of the V-shaped asphalt floor $(A F)$ transported fresh urine from the urination location to the centre of the floor. If a cow urinated while standing in a cubicle, the area covered by a urine puddle could be large, even larger than the area covered by the FOV, which was approximately $2 \mathrm{~m}^{2}$. If 
the puddle was this large, we always tried to include most of it in the FOV. From our observations we estimated that in these specific cases, the potential bias in estimating $A_{p}$ was $5 \%$ to $10 \%$ at maximum. Therefore, we maintained these IR images in our dataset without further correction, accepting that the results slightly underestimated the real $A_{p}(0)$ values for $G F$ and $A F$. If we had excluded the IR images of these large puddles, the bias would probably be larger because we then would have excluded the largest $A_{p}(0)$ values, causing a lower mean value per Farm per Season.

The factor Season did not influence the $A_{p}(0)$ value, but the FMType did. This means that Season related factors, such as farm management, temperature and diet, did not affect on the $A_{p}$. However, floor design did affect the puddle area; the closed floor FMType AF showed larger values than the other floor designs, but the other floors did not differ from each other. Therefore, the details of the design characteristics determine whether the $A_{p}$ differs or not.

The $A_{p}(0)$ value currently used by the Technical Advisory Committee (Tac-Rav) is $0.80 \mathrm{~m}^{2}$ for both a slatted floor and a solid floor with grooves or gutters, and $1.20 \mathrm{~m}^{2}$ for a solid floor without grooves or gutters. Braam et al. (1997b) reported a mean $A_{p}$ of $1.23 \mathrm{~m}^{2}$ (SD is 0.51 ) for a double-sloped floor with one central gutter, which is comparable to the $A_{p}$ we found for our $A F$. The $0.80 \mathrm{~m}^{2}$ Tac-Rav value is close to the measured $A_{p}(0)$ value of $S F R, G F$, and $S F C O$ in this study. Both the $1.20 \mathrm{~m}^{2}$ and $1.23 \mathrm{~m}^{2}$ appear to be larger than the $A_{p}(0)$ of $A F\left(1.04 \mathrm{~m}^{2}\right)$, but $A F$ was slightly underestimated as discussed above. Moreover, the $1.23 \mathrm{~m}^{2}$ had a large SD and the $1.20 \mathrm{~m}^{2}$ Tac-Rav value holds for a levelled floor, whereas $A F$ had a slope from the sides to the middle.

The $A_{p}(0)$ values used in the performed sensitivity analysis (Snoek et al., 2014b) were uniformly distributed from $0.4 \mathrm{~m}^{2}$ to $1.8 \mathrm{~m}^{2}$. In this study the $\overline{A_{p}(0)}$ values as mean per Farm ranged from $0.6 \mathrm{~m}^{2}$ to $1.1 \mathrm{~m}^{2}$ (Table 6.4 ), and at the individual puddle level, the $A_{p}(0)$ ranged from $0.2 \mathrm{~m}^{2}$ to $1.7 \mathrm{~m}^{2}$. As such the range used in the sensitivity analysis agrees with the range found in this study.

\subsubsection{Puddle depth}

In addition to PREclean (Section 6.4.4), the factor Season strongly influenced $D_{p}(0)$. One explanation for the Season effect may be that manure is dryer and stuck more to the floor in the spring period $(S)$ than in the winter period $(W)$, creating a rougher surface. The rough surface likely caused larger $D_{p}(0)$ values as manure remainders prevented urine transport on the floor.

The $D_{p}(0)$ value currently used by the Tac-Rav is $0.48 \mathrm{~mm}$ for a slatted floor or a solid floor with grooves or gutters, and $0.37 \mathrm{~mm}$ for a double-sloped $(1.5 \%)$ solid floor with a urine gutter in the middle. These values are based on literature values and expert judgement in cases where data for estimating floor effects were lacking. 
The current study showed larger $D_{p}(0)$ values, together with a large variation, for floors with similar design characteristics. The $D_{p}(0)$ for the $S F R$ without PREclean treatment $(1.4 \mathrm{~mm})$ was about 3 times larger than the Tac-Rav value of a slatted floor $(0.48 \mathrm{~mm})$. Furthermore, the $D_{p}(0)$ for the $A F$ without PREclean treatment $(1.9 \mathrm{~mm})$ was more than 5 times larger than the Tac-Rav value of a double-sloped floor $(0.37 \mathrm{~mm})$.

Our study showed that individual puddle measurements strongly varied within a Farm, with extremely high and extremely low or even negative depth values. The extremely high values were most likely caused by manure or other dirt present on the floor that created a kind of dike that held the urine in place. The described cause was common practice in most dairy cow houses within this study. The extremely low and negative values could also have been caused by manure or other dirt, in combination with the measurement method. If manure or dirt was present, the manually poured urine may have flushed away part of this manure or dirt. This may have resulted in a larger distance in the "wet" measurement compared to the "dry" measurement, which caused a negative depth value. Furthermore, this flushing may cause a systematic lower $D_{p}$ value for all $D_{p}$-measurements. Another cause may be the floor design itself. The ultrasonic device used to measure emits a sound-wave covering a surface of about $5 \mathrm{~cm}^{2}$. If a floor had a surface with several tiny grooves, it is not clear whether the distance was measured to the top layer, inside these tiny grooves, or a "mix" of these two. Finally, the puddle depth values strongly varied, depending on the floor condition, design and cleanliness. For example, we observed and measured completely full grooves at the grooved floor $(G F)$ with urine layers that varied from $10 \mathrm{~mm}$ to $50 \mathrm{~mm}$. We also observed dirty floor parts where urine was held in place by the dirt, and the $D_{p}$ were tens of millimetres as well. Since we had several puddle depth measurements per Farm and per Season, we expected to have representative mean $D_{p}$ values and distributions. Moreover, the results represent the real common practice in dairy cow houses.

The $D_{p}(0)$ values used in the performed sensitivity analysis (Snoek et al., 2014b) were uniformly distributed from $0.13 \mathrm{~mm}$ to $1.6 \mathrm{~mm}$. In this study the $\overline{D_{p}(0)}$ values as mean per Farm, without PREclean treatment, ranged from $0.89 \mathrm{~mm}$ to $2.21 \mathrm{~mm}$ (Table 6.4), and at individual puddle level the $D_{p}(0)$ ranged from $-1.8 \mathrm{~mm}$ to $5.8 \mathrm{~mm}$. Therefore, the range used in the sensitivity analysis was too small and contained too low values.

For $D_{p}$, we conclude that a two-digit mm accuracy cannot be realistically determined, and that it is difficult to define tiny differences between $D_{p}$ values. In addition, $D_{p}$ values are much larger and have wider ranges in practice than is assumed by the Tac-Rav at the current moment.

$A_{p}(0)$ and $D_{p}(0)$ showed no clear relation. The $A_{p}(0)$ and $D_{p}(0)$ measurements were not simultaneously performed at the same puddle. Because of this, mean values 
per Farm and per Season were used and the number of data points was limited. The mean data, however, represent the larger datasets of $A_{p}(0)$ and $D_{p}(0)$. Therefore, we expected that the shown data are representative for the $A_{p}-D_{p}$ relation.

\subsubsection{Floor scraping}

The manually-operated manure scraper during the $D_{p}$ measurements (PREclean) always scraped the floor more intensely, and thus more cleanly, than the commercial on-farm manure scrapers. Therefore, the PREclean results show the potential of floor cleaning with regard to $D_{p}(0)$ and the related $V_{p}(0)$. This potential is large since the $D_{p}(0)$ and $V_{p}(0)$ in the intense-cleaned situation were about $50 \%$ to $70 \%$ lower than those in the as-is situation with the on-farm scraping.

We did not measure the effects of PREcleaning on $A_{p}$. From visual observations during the measurements and by evaluating the collected urine puddle images, we suspect that the floor's cleanliness influence the $A_{p}(0)$ as well. We observed that dirty floors resulted in smaller $A_{p}(0)$ values compared to a clean floor. Dirt created a kind of dike that held the urine in place. When the floor was dirty, the urine remained on the floor until a manure scraper passed by. In short, when a floor is cleaner, a urine puddle can flow more easily and the $A_{p}(0)$ will be larger, but the puddle is also able to move more easily towards the gutters or the slurry pit.

Another effect caused by the floor's cleanliness was that fresh and still warm manure dungs were occasionally present in an IR image, which could be interpreted by the IR model as a puddle area. Often these manure dungs were covered by a fresh urine puddle. Therefore, based on expert judgement, none of these IR images were excluded.

The state of the on-farm scrapers influenced the scraping and thus cleaning quality. At all farms in this study, the manure scrapers showed signs of wear and tear, and in all cases the scraper was not able to clean the floor as completely as the manual intense-cleaning did. There were always locations on the floor that were rarely or never scraped, and dried manure made effective scraping impossible. To summarise, none of the commercial manure scrapers was able to clean the floor as thoroughly as the PREclean treatment did, and this had a profound effect on both $A_{p}$ and $D_{p}$ and ultimately on the $\mathrm{NH}_{3}$ emission. This shows that there is a considerable potential to decrease floor emissions by both improved designs for floors and scrapers, and management that minimise puddle area and depth. 
Table 6.4: The number of urine puddles (N), the mean (SD) per Farm, and the p-value to test equality of means. For variables $A_{p}(0), D_{p}(0)$, and $V_{p}(0)$.

\begin{tabular}{|c|c|c|c|c|c|c|c|c|c|c|}
\hline \multirow[b]{3}{*}{ Farm } & \multirow{2}{*}{\multicolumn{2}{|c|}{$A_{p}(0)\left[\mathrm{m}^{2}\right]$}} & \multicolumn{4}{|c|}{ PREclean $=$ of $f$} & \multicolumn{4}{|c|}{ PREclean $=$ on } \\
\hline & & & \multicolumn{2}{|c|}{$D_{p}(0)[\mathrm{mm}]$} & \multicolumn{2}{|c|}{$V_{p}(0)^{\#}\left[\mathrm{~m}^{3}\right]$} & \multicolumn{2}{|c|}{$D_{p}(0)[\mathrm{mm}]$} & \multicolumn{2}{|r|}{$V_{p}(0)^{\#}\left[\mathrm{~m}^{3}\right]$} \\
\hline & $\mathrm{N}$ & Mean (SD) & $\mathrm{N}$ & Mean (SD) & $\mathrm{N}^{\ddagger}$ & $\begin{array}{l}\text { Mean (SD) } \\
\cdot 10^{-3}\end{array}$ & $\mathrm{~N}$ & Mean $(\mathrm{SD})$ & $\overline{\mathrm{N}^{\ddagger}}$ & $\begin{array}{l}\text { Mean (SD) } \\
\cdot 10^{-3}\end{array}$ \\
\hline$F 1$ & 24 & 0.83 & 96 & $1.16(0.81)$ & 2 & 0.96 & 18 & $0.66(0.72)$ & 1 & $0.53(-)$ \\
\hline$F 2$ & 21 & $0.76(0.30)$ & 63 & $2.21(0.96)$ & 2 & $1.68(0.26)$ & 45 & $0.84(0.51)$ & 2 & $0.68(0.29)$ \\
\hline$F 3$ & 22 & $0.71(0.28)$ & 72 & $1.90(1.03)$ & 2 & $1.40(0.66)$ & 45 & $0.59(0.39)$ & 2 & 0.42 \\
\hline$F 4$ & 21 & $1.00(0.26)$ & 81 & $1.24(1.21)$ & 2 & $1.23(0.55)$ & 36 & $0.87(0.89)$ & 2 & $0.93(0.80)$ \\
\hline$F 5$ & 19 & $0.64(0.21)$ & 72 & $1.42(0.96)$ & 2 & $0.92(0.14)$ & 45 & $0.90(1.05)$ & 2 & $0.58(<0.01)$ \\
\hline$F 6$ & 21 & $1.08(0.33)$ & 99 & $1.76(1.18)$ & 2 & $1.87(0.05)$ & 18 & $0.93(0.68)$ & 1 & $0.82(-)$ \\
\hline F7 & 20 & $0.82(0.25)$ & 90 & $1.15(0.79)$ & 2 & $0.92(0.26)$ & 36 & $0.45(0.28)$ & 2 & $0.39(0.12)$ \\
\hline$F 8$ & 21 & $0.62(0.21)$ & 90 & $0.89(1.14)$ & 2 & $0.56(0.06)$ & 36 & $0.66(0.76)$ & 2 & $0.41(0.24)$ \\
\hline$F 9$ & 21 & $0.76(0.22)$ & 90 & $1.69(1.12)$ & 2 & $1.29(0.12)$ & 36 & $1.01(0.98)$ & 2 & $0.77(0.21)$ \\
\hline$F 10$ & 22 & $0.99(0.28)$ & 90 & $2.01(1.35)$ & 2 & $1.95(0.94)$ & 35 & $1.21(0.97)$ & 2 & $1.15(0.69)$ \\
\hline$F 11$ & 20 & $0.97(0.29)$ & 90 & $1.95(1.18)$ & 2 & $1.93(0.87)$ & 36 & $0.74(0.51)$ & 2 & $0.73(0.21)$ \\
\hline$F 12$ & 21 & $0.76(0.17)$ & 90 & $1.51(1.06)$ & 2 & $1.14(0.07)$ & 45 & $0.60(0.58)$ & 2 & $0.43(0.21)$ \\
\hline$F 13$ & 17 & $0.76(0.20)$ & 90 & $1.84(0.90)$ & 2 & $1.41(0.26)$ & 36 & $1.03(1.01)$ & 2 & $0.76(0.48)$ \\
\hline$F 14$ & 20 & $1.13(0.33)$ & 90 & $1.49(0.87)$ & 2 & $1.67(0.39)$ & 36 & $0.85(0.50)$ & 2 & $0.94(0.29)$ \\
\hline$F 15$ & 20 & $0.67(0.18)$ & 90 & $1.19(1.19)$ & 2 & $0.83(0.65)$ & 36 & $0.76(0.88)$ & 2 & $0.53(0.52)$ \\
\hline$F 16$ & 23 & $0.71(0.20)$ & 90 & $1.57(0.84)$ & 2 & $1.10(0.03)$ & 36 & $0.47(0.49)$ & 2 & $0.34(0.18)$ \\
\hline p-value ${ }^{\$}$ & & $<0.001$ & & $<0.001$ & & 0.133 & & $<0.001$ & & 0.709 \\
\hline
\end{tabular}

\# $V_{p}(0)$ was calculated according to Eq. (6.1) with $\Rightarrow \ddagger \mathrm{N}$ is the number of Seasons.

$\$$ p-value of REML F-statistic 


\subsection{Conclusions}

The objective of this research was to assess the puddle area $\left(A_{p}(0)\right)$, puddle depth $\left(D_{p}(0)\right)$, the resulting puddle volume $\left(V_{p}(0)\right)$, and the relations between them for fresh urine in commercial dairy cow houses.

Estimated overall mean values were the following:

- $\overline{A_{p}(0)}$ was $0.83 \mathrm{~m}^{2}$ with a large variation $(\mathrm{CV}=29.2 \%)$;

- $\overline{D_{p}(0)}$ was $1.0 \mathrm{~mm}$ with an extreme large variation $(\mathrm{CV}=79.4 \%)$;

- The resulting $\overline{V_{p}(0)}$ was $0.94 \times 10^{-3} \mathrm{~m}^{3}$ with a large variation $(\mathrm{CV}=30.1 \%)$;

For all three variables $A_{p}(0), D_{p}(0)$ and $V_{p}(0)$, the variance between Farms was negligible, whereas the variance within Farms was substantial. The CV values for $A_{p}$ and $V_{p}$ were about $30 \%$ and for $D_{p}$ about $80 \%$.

The Floor-Management type (FMType) factor significantly affected all three variables $A_{p}(0), D_{p}(0)$ and $V_{p}(0)$. For all variables the $\mathrm{V}$-shaped asphalt floor $(A F)$ resulted in significantly larger values than the subgroup of slatted floor, grooved floor and Cows \& Opportunities participants with a slatted floor. The $A F$ resulted in $1.04 \mathrm{~m}^{2}, 1.5 \mathrm{~mm}$ and $1.46 \times 10^{-3} \mathrm{~m}^{3}$, compared to the subgroup mean values of $0.76 \mathrm{~m}^{2}, 0.9 \mathrm{~mm}$ and $0.77 \times 10^{-3} \mathrm{~m}^{3}$. The PREclean factor, the intense-floor cleaning before puddle creation had strongly affected both $D_{p}(0)$ and $V_{p}(0) . D_{p}(0)$ values with PREclean treatment were about $1 / 3$ compared to the situation with commercial manure scrapers without PREclean treatment. Compared to the current practice of scraping, the potential of good floor cleaning is large. The Season factor influenced $D_{p}(0)$ as well but not the $A_{p}(0)$. In winter the $D_{p}$ was larger and the difference with the spring value was $0.32 \times 10^{-3} \mathrm{~m}^{3}$.

\subsection{Acknowledgements}

The authors would like to thank the 16 dairy farmers involved in the study for their hospitality, cooperation during measurements, and for their contributions to the discussions on this research. In addition, the authors would also like to thank W. Bin, S. Bomers, A. van Dueren den Hollander, G. Kupers, and R. Vernooij for their assistance during the measurements. Finally, the authors would like to thank M. Kluivers-Poodt for facilitating the use of the IR camera. Language editing of a near-final draft of the paper was provided by P. Griffith.

\subsection{Supplementary Material}

see Table 6.4. 

Assessing fresh urine puddles in commercial dairy cow houses part 2: $\mathrm{pH}$, urea concentration, and ammonia emission potential

Dennis Snoek

Hans Stigter

Geert Kupers

Peter Groot Koerkamp

Nico Ogink

Submitted for Publication 


\begin{abstract}
In The Netherlands, new mitigation methods to reduce ammonia emission in dairy cow houses are pre-assessed and regulated by a technical committee (Tac-Rav) using a mechanistic emission model. However, required model inputs have not been updated since 2000. In addition, values for the model variables $p H$, Urinary Urea Nitrogen concentration $(U U N)$, and their relation with farm and feed management are unknown for commercial dairy cow houses. Moreover, their effect on ammonia emission is unknown. Therefore, the objective of this paper was to investigate the $p H$ and $U U N$ in livestock practice. Sixteen commercial farms were measured in a factorial design of four Floor-Management types (FMTypes). Each farm was measured in two seasons and a Diet factor was defined, based on the amount of grass in total roughage. Overall mean values were $4.27 \mathrm{~kg} \mathrm{~m}^{-3}$ for $U U N$, an initial $p H$ of 8.3 , both in fresh puddles, and a $p H(\xi)$ of 9.0 for random puddles at a random time. For $U U N$ both the variation within and between farms was large, whereas the variation for $p H$ was small. The Diet was the only factor that resulted in a significant effect, with a 0.1 difference in $p H(\xi)$. Compared to the Tac-Rav reference values, both the mean $U U N$ and $p H$ showed smaller values. The calculated potential ammonia in $\mathrm{kg}_{\text {puddle }}{ }^{-1}$ showed a huge range and was considerably larger than the Tac-Rav reference values.
\end{abstract}




\subsection{Introduction}

Ammonia $\left(\mathrm{NH}_{3}\right)$ emission strongly contributes to the acidification of the environment. To curtail this, the EU has set National Emission Ceilings (NEC) (EU, 2001) that substantially reduced $\mathrm{NH}_{3}$ emission in Europe from the first reported values in 1990 until 2010 (EEA, 2012). This decrease was also observable in The Netherlands. However, in 2010 to 2013 the Dutch $\mathrm{NH}_{3}$ emission still exceeded the NEC (EEA, 2015). In Europe, the most important contributor to the $\mathrm{NH}_{3}$ emission is agriculture, with a share of $95 \%$ (EEA, 2015). From this $95 \%$, in The Netherlands, the $\mathrm{NH}_{3}$ emission from dairy cow houses is one of the largest sources (Velthof et al., 2012). In these cow houses, the $\mathrm{NH}_{3}$ emission originates from urea in the urine on top of the floor and from urea in the slurry in the slurry pit beneath the floor. The emission ratio between floor and pit is about 70:30 \% according to Monteny et al. (1998).

To lower $\mathrm{NH}_{3}$ emissions from dairy cow houses, Dutch farmers are legally obliged to use $\mathrm{NH}_{3}$ emission mitigation methods. New methods and their application are assessed, approved and regulated by a Technical Advisory Committee (Tac-Rav, 2016). Experts in the Tac-Rav use a model developed by Monteny et al. (1998). With the Tac-Rav model, the $\mathrm{NH}_{3}$ emission of a cow house is estimated based on a set of input variables. The Tac-Rav use the model to pre-assess new mitigation methods and, related, new low-emission cow houses to give them a provisional emission factor. The provisional factor will be replaced by a definite factor after full-scale $\mathrm{NH}_{3}$ emission measurements have been performed according to the prescribed protocol (Ogink et al., 2013). However, the model from Monteny et al. (1998) was initially designed for research purposes and not for pre-assessing full scale commercial dairy cow houses. The model requires estimates of several variables that are representative for practical conditions in a cow house such as the puddle urea concentration. Values for these model variables are scarce and are not regularly measured, since they are not included in the protocol (Ogink et al., 2013). Moreover, input values for the model associated with new mitigation methods are difficult to determine or to assess with the model. Therefore, using the Tac-Rav model has its limitations, and it is a complex exercise to assess new mitigation methods and thus new low-emission houses. A simpler model is desirable.

To develop a simplified, better usable $\mathrm{NH}_{3}$ emission model, we first analysed the current Tac-Rav model in a sensitivity analysis along with comparable emission models from other studies (Snoek et al., 2014b). In this study, we concluded that the five most import input variables to estimate the $\mathrm{NH}_{3}$ emission were the $\mathrm{pH}$, urea concentration, depth, area and temperature of a urine puddle. The model parameters did not show an effect. It was also concluded that data for these urine puddle variables were hardly available from commercial dairy cow houses. These data are necessary to give better advice to the Tac-Rav to be able to develop a more simple, yet accurate 


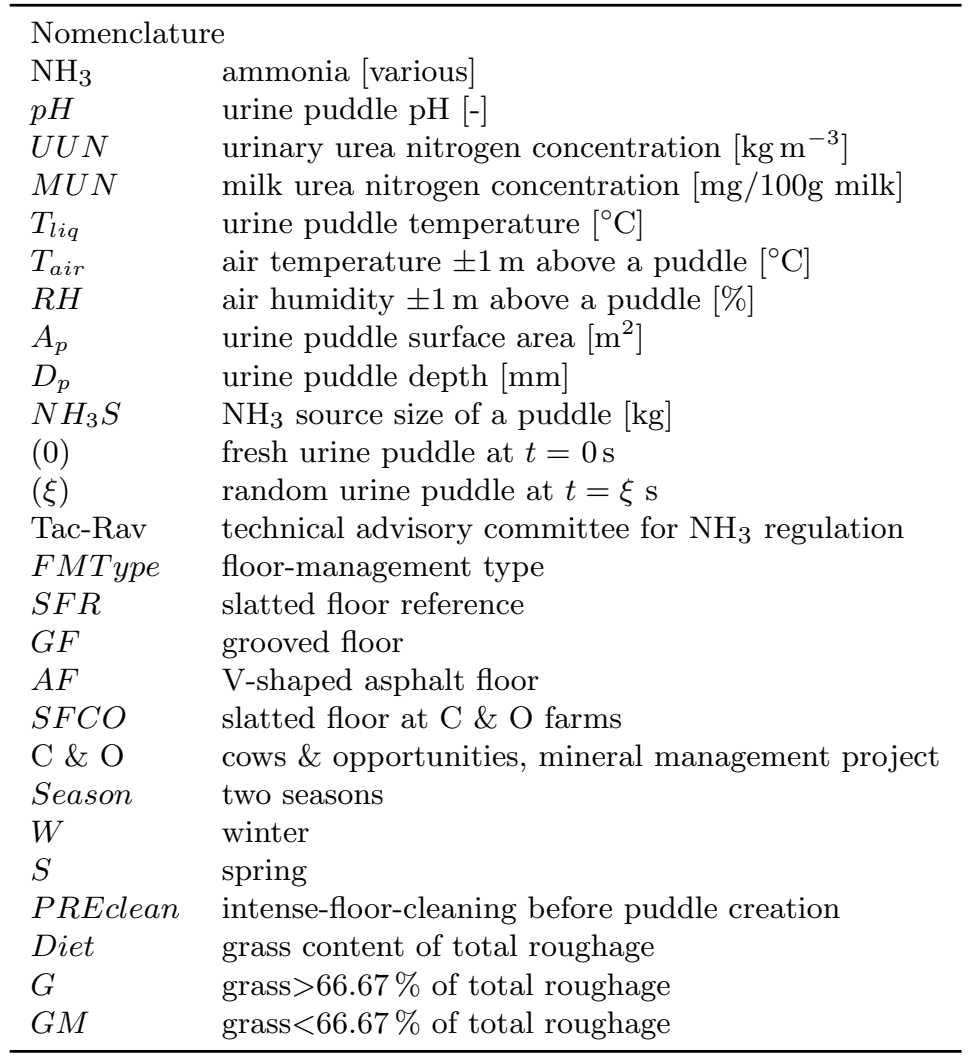

$\mathrm{NH}_{3}$ emission model and to validate it. To obtain these data from dairy cow houses and to understand possible relations and effects under practical circumstances, we set up a measurement series. In this series we simultaneously measured the five most important puddle variables of fresh urine puddles on floors in commercial cow houses.

Fresh dairy cow urine puddles were measured at sixteen farms, based on floor type and farm management, and in two seasons. The farms, their related feed management, and the local climate were diverse, resulting in varying chemical characteristics of the urine, for example, different $p H$ and urinary urea nitrogen concentration (UUN) values (van Duinkerken et al., 2011; Monteny et al., 2002) and varying puddle and air temperatures and air humidity. The milk urea nitrogen concentration $(M U N)$ also varies, as a result of different feed management. Several authors have demonstrated positive relationships between $M U N$ and $U U N$ (van Duinkerken et al., 2011; Burgos et al., 2007). In commercial dairy cow houses, however, Burgos et al. (2005) did not find correlations between $M U N$ and $U U N$. That said, in The Netherlands it is expected that $\mathrm{NH}_{3}$ emission can be lowered by adjusting the feed management. 
Besides, recently a tool to visualise the effect of farm management on a farm's nutrient cycle was introduced. The Annual Nutrient Cycling Assessment (ANCA) is a "license to produce" for dairy farms with a manure surplus from 2015 onwards (Aarts et al., 2015). But to the best of our knowledge, the $p H$ and $U U N$ values and the extent of the effects by farm \& feed management and climate on them are unknown for commercial dairy cow houses. Therefore, we focused in this paper on farm management and local climate in relation to the chemical characteristics of a urine puddle.

The results from our field study are reported in two papers. The results of the physical characteristics of a urine puddle: the surface area and depth, are described in Snoek et al. (2016c). The objective of the current paper is to investigate the Urinary Urea Nitrogen concentration $(U U N)$, the related potential $\mathrm{NH}_{3}$ source size, the initial $p H(p H(0))$, the $p H$ at a random moment $(p H(\xi))$, the puddle and air temperature

$\left(T_{l i q}, T_{a i r}\right)$, and the relations between them in commercial dairy cow houses. The Milk Urea Nitrogen concentration $(M U N)$ was also obtained to relate it to the $U U N$. Finally, the effect of floor type, season and feed management on these variables were also analysed.

\subsection{Materials and Methods}

\subsubsection{Experimental design}

The experimental design is described in more detail in Snoek et al. (2016c).

\section{Design and experimental factors}

In total, we measured urine puddle characteristics at 16 commercial dairy farms, spread over The Netherlands. These farms were divided into a factorial setup over four Floor-Management types (FMTypes). Each FMType consisted of four farms. The FMTypes were (1) slatted floor farms comparable to the reference cow house type $(S F R)$, (2) completely closed grooved floor farms $(G F),(3)$ completely closed $\mathrm{V}$-shaped asphalt floor farms $(A F)$, and (4) slatted floor farms that participated in the Cows \& Opportunities project $(S F C O)$. Cows \& Opportunities farmers actively manage the nutrient cycle of the cows to lower $\mathrm{NH}_{3}$ emission (Oenema et al., 2001; Aarts et al., 2015). The two pre-set experimental factors were Season, which represents the measurements in winter $(W)$ and spring $(S)$, and PREclean, which represents the simulated intensively-cleaned-floor-effect before puddle creation on the $D_{p}$ measurements as compared to on-farm manure scraping if present (Snoek et al., 2016c). Each farm was measured in both Seasons, and PREclean was applied at each farm. Additionally, there were two possible explanatory factors distinguished: Diet and Calc. These were not controlled at farm level and were not equally spread among 
the FMTypes. The factor Diet represents an indication of the feed management at the farm, with grass $>66.67 \%$ of total roughage $(G ; 8$ farms) and with grass $<66.67 \%$ of total roughage ( $G M ; 8$ farms). Roughage is the amount of grass and corn in the total diet. The second explanatory factor Calc represents the use of calcium in the cubicle ( 5 farms with and 11 farms without). Each farm was measured both in winter and in spring, and the order of farm measurements was randomised per season. Each farm measurement lasted for a single day and started in the morning between nine and ten o clock. Before and during the measurements, the cows were inside the cow house.

\section{Dairy farm characteristics}

The majority of the cow houses were built within the last 5 yrs to $10 \mathrm{yrs}$ with an average age of about $5 \mathrm{yrs}$ and most of the houses contained an automatic milking system (AMS). Additionally, almost all cow houses contained a manure scraper that was either a conventional pulled scraper, or a scraper robot. The scrape intensity varied from 2 to 12 times a day. There were two main cubicle bedding types, a mattress of soft material or water combined with sawdust, or a deep litter cubicle with sand, straw or dried manure. Five farmers used a calcium additive (Calc) in their bedding material. Four of those five farmers added calcium to their sawdust, and one to the straw. A more detailed description of the dairy farms can be found in Snoek et al. (2016c).

Detailed feed intake and nutritional information were not measured in the current study. Each farmer, however, was asked about their feed management during the measurement day. The information consisted of the amounts and types of feed they fed to the dairy cows. With this feed information we defined the factor Diet, which is based on the grass content of the roughage in $\mathrm{kg}$ (Section 7.2.1).

\subsubsection{Measurements}

\section{Measured variables}

Measurements were carried out at different groups of puddles in three consecutive steps: investigating fresh puddles, present puddles on the floor, and manually created puddles. At each farm we first measured 10 fresh urine puddles directly after the cow had finished urinating $(t=0 \mathrm{~s})$. At $t=0 \mathrm{~s}$ we measured $p H(0)$, the urinary urea nitrogen concentration $(U U N(0))$ and the temperature $\left(T_{\text {liq }}(0)\right)$ of these puddles, and the local air temperature $\left(T_{a i r}(0)\right)$, and the relative humidity $(R H(0))$ just above them. In addition to these variables, we measured the puddle area $\left(A_{p}(0)\right)$ at $t=0 \mathrm{~s}$ ((Snoek et al., 2016c)). After measuring the fresh puddles, we measured 10 randomly selected urine puddles that were already present on the floor for a random time period $(t=\xi \mathrm{s})$. We measured the $p H(\xi)$, and the temperature $\left(T_{l i q}(\xi)\right)$ of these random 
puddles, and the local air temperature $\left(T_{\text {air }}(\xi)\right)$, and the relative humidity $(R H(\xi))$ just above them. Finally, we measured the puddle depth $\left(D_{p}(0)\right)$ of 63 puddles that were manually created with clean fresh urine collected at the same farm ((Snoek et al., 2016c)). Afterwards, we obtained the milk urea nitrogen concentration $(M U N)$ for each farm. The $M U N$ was determined by the dairy company that collects the milk every three days. Therefore, the $M U N$ value was the average of three days of milk from the whole farm, and was determined one to three days before the actual measurement day.

\section{Equipment}

To measure $p H, T_{l i q}, T_{a i r}$, and $R H$, we used a custom-made sensor-rod (Fig. 7.1). The sensor-rod consisted of a fast-responding $T$ sensor (Testo AG, GER) attached to the $p H$ sensor (Horiba Ltd, JPN), both of which were connected to the rod by a small chain. On the rod, a portable $p H / O R P$ meter (Horiba Ltd, JPN), a $T / R H$ sensor, and a multifunction meter (Testo AG, GER) were attached. All variables were measured with a time interval of $2 \mathrm{~s}$, which was the fastest option for the $p H / O R P$ meter. More details about and the accuracy of the sensors can be found in Snoek et al. (2016b). The tripod described in Snoek et al. (2016b) was not used in this study. Instead, we used a rod to be able to move quickly and flexibly and to put the sensors in a puddle from a distance; otherwise, we could have scared cows and caused them to kick or run away.

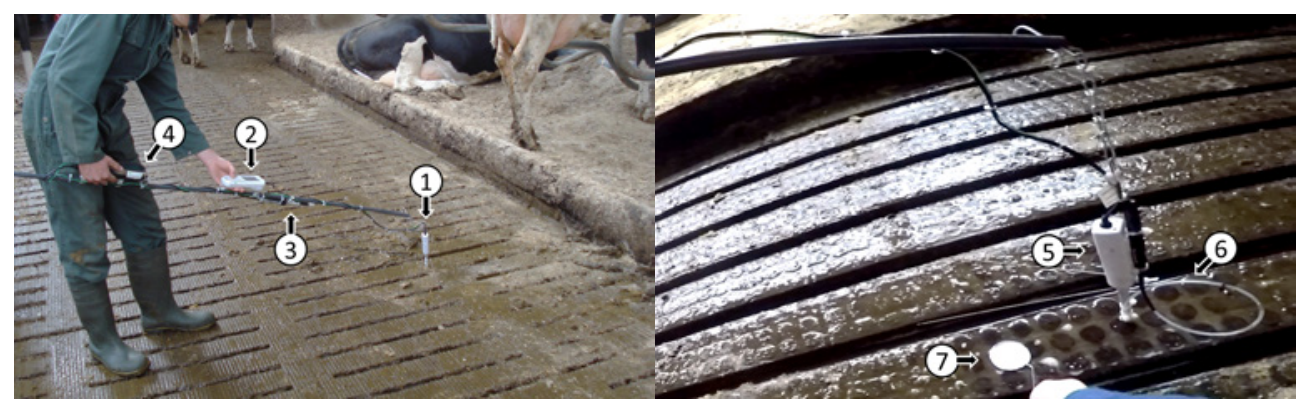

Figure 7.1: The sensor-rod with on the left (1) the $p H$ and $T_{l i q}$ sensors, (2) $p H / O R P$ meter, (3) $T_{a i r} / R H$ sensor, (4) $T_{l i q} / T_{a i r} / R H$ multifunction meter, and on the right (5) the $p H$ sensor, (6) the $T_{l i q}$ sensor in protection tube, and (7) the filter to take a urine sample. For sensor details see Snoek et al. (2016b).

To measure $U U N$, we adapted the method developed by Aarnink et al. (2015). Puddle samples of fresh urine were collected with circular glass fibre filters with a $47 \mathrm{~mm}$ diameter (Macherey-Nagel, MN GF-3) that worked like a sponge (Fig. 7.1). After collection, the urine-soaked filter was placed in a circular jar with $10 \mathrm{ml}$ of $1 \mathrm{M}$ 
hydrochloric acid (HCL) to immediately stop the conversion process of urea to $\mathrm{NH}_{3}$. The filter papers, the jars, and the amount of acid were individually prepared and weighed in the lab (Mettler Toledo, DeltaRange PG503-S, with an accuracy of $0.002 \mathrm{~g}$ ). The preparation was done in batches of 40 to 80 jars at once and from one day or a few weeks before a measurement day. The prepared jars were stored in a refrigerator until use. On the morning of a measurement day, 10 jars were removed from the refrigerator and stored in a cool box with cooling elements for transport. After a measurement day, the total weight of each jar, including the filter, the acid, and the urine sample, was measured and the urine sample weight $(S W)$ was then calculated. The amount of acid divided by $S W$ resulted in the sample dilution ( $S D i l)$. The jars with urine sample were stored in a refrigerator. Lab analyses of the samples to determine the $\mathrm{NH}_{4}^{+}-\mathrm{N}$ (Section 7.2.2) were performed in two batches, first after the winter series and second after the spring series.

\section{Measurement protocol and calibration}

The measurement protocol is described in detail in Snoek et al. (2016c). To obtain the $p H(0), T_{l i q}(0), T_{a i r}(0)$, and $R H(0)$, we measured each fresh urine puddle for $60 \mathrm{~s}$. The variable values at $t=30 \mathrm{~s}$ were then selected. To obtain the $p H(\xi), T_{l i q}(\xi), T_{a i r}(\xi)$, and $R H(\xi)$, we measured each random puddle for $20 \mathrm{~s}$. The variable values at $t=20 \mathrm{~s}$ were then selected.

As in Snoek et al. (2016b), a two-point calibration of the $p H$ sensor was performed on-site, at the start of each measurement day. We used two calibration buffer solutions: $7.01 \pm 0.01$ and $10.01 \pm 0.01$ (Hanna Instruments ${ }^{\circledR}$, USA). The Testo sensors were calibrated in the lab by using a F250 Precision Thermometer $\left( \pm 0.005^{\circ} \mathrm{C}\right.$, ASL, USA). The $T_{l i q}$ measured by the Testo sensor was used to re-correct the $p H$ afterwards (Snoek et al., 2016b). After each puddle measurement the $p H$ sensor was rinsed with demineralised water.

\section{Lab analysis}

The jars with collected urine (Section 7.2.2) were analysed in the lab to obtain $U U N$ values. In the lab a urease solution was prepared that consisted of $60 \mathrm{mg}$ urease added to $100 \mathrm{ml}$ phosphate buffer solution. The urease originated from beans, and had a catalytic activity of $5 \mathrm{U} \mathrm{mg}^{-1}$. The phosphate buffer solution consisted of $14.3 \mathrm{~g} \mathrm{KH} \mathrm{KO}_{4}$ and $90.0 \mathrm{~g} \mathrm{~K} \mathrm{HPO}_{4} \cdot 3 \mathrm{H}_{2} \mathrm{O}$, dissolved in $1 \mathrm{l}$ demineralised water. The analysis consisted of four consecutive steps:

1. Dilute the sample with demineralised water to fit the calibration curve. Two dilutions are created: 1000x and 2000x;

2. Determine the initial $\mathrm{NH}_{4}^{+}-\mathrm{N}$ concentration of a sample, which is ideally zero; 
3. Add the urease solution to increase the sample $p H$ to re-start the urea conversion process. Dilute twice with the urease solution $(750 \mu l)$;

(a) Leave the dilutions for $1 \mathrm{~h}$, after which all the urea is converted to $\mathrm{NH}_{4}^{+}-\mathrm{N}$;

(b) If all went well, the $\mathrm{NH}_{4}^{+}-\mathrm{N}$ concentration of the $1000 \mathrm{x}$ dilution is twice the concentration of the $2000 \mathrm{x}$ dilution;

4. Check the urease activity of the dilutions and determine the $\mathrm{NH}_{4}^{+}-\mathrm{N}$.

$\mathrm{NH}_{4}^{+}$-N was determined according to the NEN-ISO 7150:2002EN (Dutch Normalisation Institute, 2002). The obtained $\mathrm{NH}_{4}^{+}-\mathrm{N}$ concentration difference between the start and the end of the sample was used to determine the urea concentration of the diluted sample $\left(U U N_{d i l}\right)$ with equations (1) and (4) from Snoek et al. (2014b). By multiplying the start $\mathrm{NH}_{4}^{+}-\mathrm{N}$ concentration by the sample dilution $S$ Dil, the $\mathrm{NH}_{4}^{+}-\mathrm{N}$ sample concentration was obtained. By multiplying the $U U N_{d i l}$ by the sample dilution $S D i l$, the $U U N$ was obtained.

\subsubsection{Data analyses}

\section{Incorrect values}

None of the data were determined as incorrect in the $t=0 \mathrm{~s}$ or $t=\xi \mathrm{s}$ datasets for the measured $p H, T_{l i q}, T_{a i r}$, and $R H$.

The sample weight $S W$ and related sample dilution $S D i l$ were needed to determine $U U N$ (Section 7.2.2). The $S W$ was negative for four jars in total. This was probably caused by a loss of acid, fresh urine, or a combination of them. These four samples were excluded from the dataset. In addition, it was unknown how many jars may have lost some fluid or contained an additional amount of dirt and how large the resulting $S W$-error was per sample. As a result, the $S D i l$ may not have been correct for some samples, causing some implausible $U U N$ values. Therefore, incorrect values were excluded based on the relationship between the determined $U U N$ and the urea concentration of the diluted sample $\left(U U N_{d i l}\right)$, as shown in Fig. 7.2. A Generalised Linear Model was applied to this scatter plot, which was log-linear, and a log-link was applied. The Pearson residuals mean value and the $95 \%$ confidence intervals were then determined. The remaining outlying $5 \%$ data were selected (15 data points) and excluded. Finally, to verify the selection, we plotted the residuals against the $U U N_{d i l}$, including the residuals mean-value-line and $95 \%$ confidence intervals (not shown). 


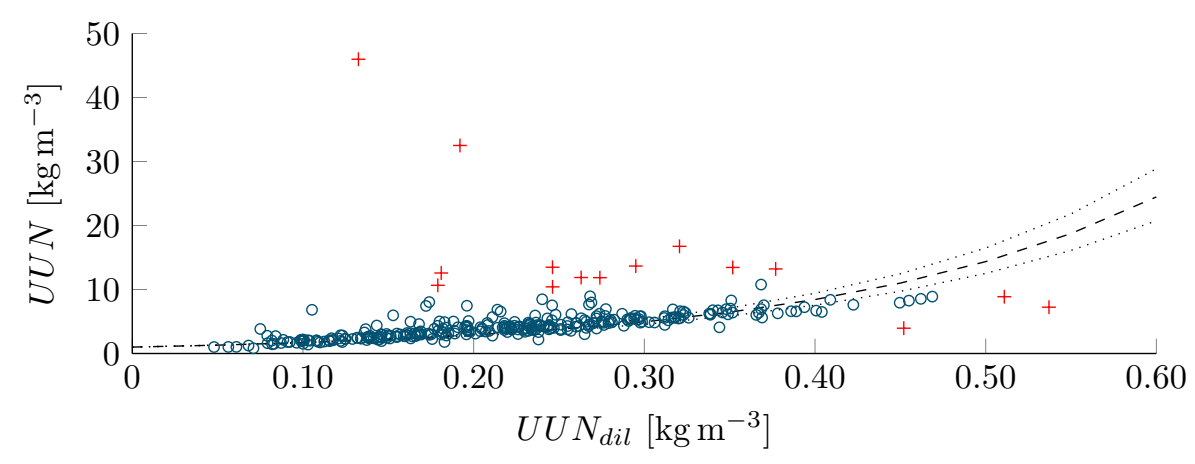

Figure 7.2: $U U N(0)$ vs $U U N_{d i l}\left({ }^{\circ}\right)$, with the log-linear GLM (---), $95 \%$ prediction intervals $(\cdots \cdots)$, and the identified incorrect values $(+)$.

\section{Calculation of $\mathrm{NH}_{3}$ source size}

From the variables $A_{p}(0)\left[\mathrm{m}^{2}\right], D_{p}(0)[\mathrm{mm}]$ (Snoek et al., 2016c), and $U U N(0)\left[\mathrm{kg} \mathrm{m}^{-3}\right]$, the $\mathrm{NH}_{3}$ source size $\left(\mathrm{NH}_{3} \mathrm{~S}(0)\right)$ [kg] was calculated according to Eq. (7.1):

$$
\overline{N H_{3} S(0)}=\overline{A_{p}(0)} \cdot \overline{D_{p}(0)} \cdot 10^{-3} \cdot \overline{U U N(0)} \cdot \frac{17}{14}
$$

with $\frac{17}{14}$ the conversion from $\mathrm{NH}_{3}-\mathrm{N}$ to $\mathrm{NH}_{3}$ (Monteny et al., 1998). Since the $A_{p}(0)$ and $U U N(0)$ were not measured at the same time in the same puddle as $D_{p}(0)$, we used the mean value per farm and per Season. For $D_{p}(0)$ we also distinguished between the data with and without PREclean treatment. This resulted in a total of $16 \cdot 2 \cdot 2=64 \mathrm{NH}_{3} S(0)$ values, where two values were missing because the PREclean treatment was not applied in Season $W$ at farms $F 1$ and $F 6$.

\section{Statistical analyses}

We performed a restricted maximum likelihood method (REML) (Payne et al., 2015) to study group effects. The models used are given in Eq. (7.2) for $p H(0), p H(\xi)$, or $U U N(0)$ represented by $y_{i j k}$, and in Eq. (7.3) for $N H_{3} S(0)$.

$$
\begin{aligned}
y_{i j k} & =\mu+T y_{p}+S e_{r}+F e_{s}+C a_{t}+F a_{i}+S e_{i j}+\epsilon_{i j k} \\
N H_{3} S_{i j l}(0) & =\mu+T y_{p}+C l_{q}+S e_{r}+F e_{s}+C a_{t}+F a_{i}+S e_{i j}+\epsilon_{i j l}
\end{aligned}
$$

With the fixed part of the model:

- $\mu$ the overall constant (grand mean),

- $T y_{p}$ the main effect of FMType (with $p$ the FMType assigned to $i j k l$ ), 
- $C l_{q}$ the main effect of PREclean (with $q$ the PREclean assigned to $i j k l$ ),

- $S e_{r}$ the main effect of Season (with $r$ the Season assigned to $i j k l$ ),

- $F e_{s}$ the main effect of Feed (with $s$ the Feed assigned to $i j k l$ ),

- $C a_{t}$ the main effect of Calc (with $t$ the Calc assigned to $i j k l$ ).

The random part of the model:

- $F a_{i}$ the effect of Farm $i(i=1 . .16)$,

- $S e_{i j}$ the effect of Season $j$ ( $j=$ winter, spring) within Farm $i$,

- $\epsilon_{i j}$. the residual error:

- for unit $i j k$ in Eq. (7.2), representing the Puddle $k(k=1 . .10)$ within Season $i j$.

- for unit $i j l$ in Eq. (7.3), representing the variance $l(l=-)$ within Season $i j$.

We estimated the variance components of each random term, the related coefficient of variation $(\mathrm{CV})$, and the Wald-statistic for the fixed terms. The variance component measures the variability of a term over and above the variability of the sub-units of which it is composed (Payne et al., 2015). The CV is the square root of the variance component, divided by the mean response $(\mu)$ times 100 .

The hypothesis $\left(h_{0}\right)$ was tested for all three variables. The values of a variable from hypothesis $\left(h_{0}\right)$ were from populations with the same mean per group compared to the alternative hypothesis $\left(h_{1}\right)$ where the population means were not the same per group. The predicted means were computed, and if $h_{0}$ was rejected, the approximate least significant differences (LSDs) were computed for these predicted means of the fixed terms to identify the statistical significant differences between subgroups. All tests were calculated by using a significance level of $\alpha=0.05$.

Subsequently, general statistics were given per FMType and per Season for $T_{l i q}(0)$, $T_{a i r}(0), R H(0), T_{l i q}(\xi), T_{a i r}(\xi)$, and $R H(\xi)$.

Finally, scatter plots were made for all kinds of variable combinations, for example $p H(0)$ vs. $U U N(0)$. For each plot a linear regression was carried out between the two variables.

\subsection{Results}

\subsubsection{Statistical analyses}

Tables 7.1 to 7.3 show the results of the REML analysis.

Table 7.1 shows that for $p H(0)$ the between-Farm variance is negligible and that the within-Farm variance is small $(\mathrm{CV}=<3.0 \%)$. None of the fixed terms showed 
a statistically significant effect $(\mathrm{p}<0.05)$. Therefore, we simplified the model from Eq. (7.2) into Eq. (7.4) by dropping terms:

$$
p H_{i j k}(0)=\mu+T y_{q}+F a_{i}+P u_{i j}+\epsilon_{i j k}
$$

with symbols according to Eq. (7.2) (Section 7.2.3). The effect of FMType was still taken into account since it represented the experimental design. Table 7.2 shows that the grand mean $p H(0)$ was 8.31, with slightly smaller values for $S F R, G F$ and $A F$, and slightly larger values for $S F C O$. The s.e. values for $p H(0)$ were small compared to the mean values, which confirms a precise estimate and the low variance.

Table 7.1: The Variance Component (Var) \& Coefficient of Variation (CV) for the defined random terms and the residual $\epsilon$, followed by the F-statistic \& p-value for the fixed terms. For $p H(0), p H(\xi), U U N(0)$, and the $\mathrm{NH}_{3}$ source $\mathrm{NH}_{3} S(0)$.

\begin{tabular}{|c|c|c|c|c|c|c|c|c|}
\hline \multirow[b]{2}{*}{ Term } & \multicolumn{2}{|c|}{$p H(0)[-]$} & \multicolumn{2}{|c|}{$p H(\xi)[-]$} & \multicolumn{2}{|c|}{$U U N(0)\left[\mathrm{kg} \mathrm{m}^{-3}\right]$} & \multicolumn{2}{|c|}{$\mathrm{NH}_{3} S(0)^{\dagger}\left[\mathrm{kg}\right.$ puddle ${ }^{-1}$} \\
\hline & $\begin{array}{r}\operatorname{Var}(\text { s.e.) } \\
\cdot 10^{-2}\end{array}$ & $\mathrm{CV}$ & $\begin{array}{r}\operatorname{Var}(\text { s.e. }) \\
\cdot 10^{-2}\end{array}$ & $\mathrm{CV}$ & Var (s.e.) & $\mathrm{CV}$ & $\begin{array}{r}\operatorname{Var}(\text { s.e.) } \\
\cdot 10^{-6}\end{array}$ & $\mathrm{CV}$ \\
\hline$F a_{i}$ & $-1.19(0.893)$ & - & $-1.86(0.894)$ & - & $1.12(0.631)$ & 24.8 & $0.77(1.685)$ & 16.6 \\
\hline$S e_{i j}$ & $3.87(1.571)$ & 2.4 & $4.25(1.709)$ & 2.3 & $0.60(0.284)$ & 18.1 & $3.37(1.977)$ & 34.7 \\
\hline \multirow[t]{2}{*}{ Residual $\epsilon$} & $5.99(0.487)$ & $2.9^{\ddagger}$ & $4.29(0.385)$ & $2.3^{\ddagger}$ & $1.622(0.140)$ & $29.8^{\ddagger}$ & $3.57(0.937)$ & $35.7 \#$ \\
\hline & F-stat & $\mathrm{p}-\mathrm{val}^{\$}$ & F-stat & $\mathrm{p}-\mathrm{val}^{\$}$ & F-stat & $\mathrm{p}-\mathrm{val}^{\$}$ & F-stat & $\mathrm{p}-\mathrm{val}^{\$}$ \\
\hline FMType & 2.11 & 0.152 & 3.45 & 0.067 & 0.80 & 0.516 & 3.81 & 0.040 \\
\hline PREclean & & - & & - & & - & 36.57 & $<0.001$ \\
\hline Season & & - & & - & 3.92 & 0.067 & 6.71 & 0.020 \\
\hline Diet & - & - & 9.42 & 0.014 & & - & & - \\
\hline
\end{tabular}

\rceil $\mathrm{NH}_{3} \mathrm{~S}(0)$ was calculated according to Eq. (7.1).

$\ddagger$ the residual represent the Puddle level.

\# the residual represent "within farm" variance.

$\$$ p-value when dropping the individual term from the full fixed model.

For the $p H_{\xi}$ the results were comparable to $p H(0)$. Table 7.1 shows that for $p H(\xi)$ the between-Farm variance is negligible and that the within-Farm variance is small $(\mathrm{CV}=<2.5 \%)$. For $p H(\xi)$ the fixed term Diet showed a statistical significant effect $(\mathrm{p}<0.05)$ and FMType was close to statistically significant $(\mathrm{p}=0.067)$. The effect of Season and Calc were not statistically significant. Therefore, we simplified the model from Eq. (7.2) into Eq. (7.5):

$$
p H_{i j k}(\xi)=\mu+T y_{q}+F e_{s}+F a_{i}+P u_{i j}+\epsilon_{i j k}
$$

with symbols according to Eq. (7.2) (Section 7.2.3). The effect of FMType was still taken into account since it represented the experimental design. Table 7.2 shows that the grand mean $p H(\xi)$ was 9.00 , with slightly smaller values for $G F$ and $A F$ and 
Table 7.2: The number of samples (N) and the predicted means with s.e., per FMType, $P R E c l e a n$, Season or Diet, and the grand mean $\mu$. For $p H(0), p H(\xi), U U N(0)$, and the $\mathrm{NH}_{3}$ source $\mathrm{NH}_{3} S(0)$.

\begin{tabular}{|c|c|c|c|c|c|c|c|c|}
\hline \multirow[b]{2}{*}{ Term } & \multicolumn{2}{|r|}{$p H(0)[-]$} & \multicolumn{2}{|r|}{$p H(\xi)[-]$} & \multicolumn{2}{|c|}{$U U N(0)\left[\mathrm{kg} \mathrm{m}^{-3}\right]$} & \multicolumn{2}{|c|}{$\mathrm{NH}_{3} S(0)^{\dagger}\left[\mathrm{kg} \mathrm{puddle}^{-1}\right]$} \\
\hline & & Mean (s.e.) & & Mean (s.e.) & & Mean (s.e.) & $\mathrm{N}^{\ddagger}$ & $\begin{array}{l}\text { Mean (s.e.) } \\
\cdot 10^{-3}\end{array}$ \\
\hline$\mu$ & 334 & $8.31(0.0255)$ & 277 & $9.00(0.0197)$ & 301 & $4.27(0.3070)$ & 62 & $5.297(0.5593)$ \\
\hline \multicolumn{9}{|c|}{-FMType- } \\
\hline$S F R$ & 91 & $8.28(0.050)$ & 72 & $9.02(0.037)$ & 73 & $4.16(0.614)$ & 15 & $3.50(1.126)^{\mathrm{a}}$ \\
\hline$G F$ & & $8.25(0.051)$ & 60 & $8.94(0.045)$ & 79 & $3.54(0.613)$ & 16 & $3.87(1.111)^{\mathrm{a}}$ \\
\hline$A F$ & & $8.29(0.051)$ & 80 & $8.95(0.036)$ & 73 & $4.70(0.615)$ & 15 & $8.32(1.126)^{\mathrm{b}}$ \\
\hline$S F C O$ & 80 & $8.42(0.051)$ & 65 & $9.10(0.040)$ & 76 & $4.69(0.614)$ & 16 & $5.50(1.111)^{\mathrm{a}, \mathrm{b}}$ \\
\hline \multicolumn{9}{|c|}{-PREclean- } \\
\hline off & & - & - & - & & - & 32 & $7.08(0.625)^{\mathrm{b}}$ \\
\hline on & & - & - & - & & - & 30 & $3.52(0.639)^{\mathrm{a}}$ \\
\hline \multicolumn{9}{|c|}{-Season- } \\
\hline$S$ & & - & - & - & 145 & $4.58(0.345)$ & 32 & $6.57(0.739)^{\mathrm{b}}$ \\
\hline \multicolumn{9}{|l|}{-Diet- } \\
\hline$G M$ & & - & 137 & $9.06(0.029)^{\mathrm{b}}$ & & - & & - \\
\hline
\end{tabular}

\rceil $\mathrm{NH}_{3} \mathrm{~S}(0)$ calculated according to Eq. (7.1) $\Rightarrow \ddagger \mathrm{N}$ is the number of Farm $\cdot$ Season $\cdot$ PREclean. $\mathrm{a}, \mathrm{b}$ statistical significant subgroups based on the approximate LSD (0.05 level) of REML means.

Table 7.3: The number of samples $(\mathrm{N})$ and the predicted means of $\mathrm{NH}_{3}$ source with s.e. per FMType PREclean. This interaction was not statistically significant. For the main effects see Table 7.1.

\begin{tabular}{|c|c|c|c|}
\hline \multicolumn{2}{|c|}{ Group } & \multicolumn{2}{|c|}{$\mathrm{NH}_{3} S(0)^{\dagger}\left[\mathrm{kg} \mathrm{puddle}^{-1}\right]$} \\
\hline FMType & PREclean & $\mathrm{N}$ & $\begin{array}{c}\text { Mean (s.e.) } \\
\cdot 10^{-3}\end{array}$ \\
\hline$S F R$ & $F$ & 8 & $5.28(1.054)$ \\
\hline$S F R$ & $T$ & 7 & $1.72(1.070)$ \\
\hline$G F$ & $F$ & 8 & $5.65(1.046)$ \\
\hline$G F$ & $T$ & 8 & $2.09(1.046)$ \\
\hline$A F$ & $F$ & 8 & $10.10(1.054)$ \\
\hline$A F$ & $T$ & 7 & $6.54(1.070)$ \\
\hline$S F C O$ & $F$ & 8 & $7.28(1.046)$ \\
\hline$S F C O$ & $T$ & 8 & $3.72(1.046)$ \\
\hline
\end{tabular}

slightly larger values for $S F R$ and $S F C O$. The table also show that the $p H(\xi)$ was larger for a Diet with grass $<66.67 \%(G M)$ of total roughage compared to grass 
$>66.67 \%(G)$ of total roughage. However, the difference was only 0.1. The s.e. values for $p H(\xi)$ were small compared to the mean values, which confirms a precise estimate and the low variance.

Table 7.1 shows that the variance for $U U N(0)$ was larger compared to the $p H$ data, with a CV of about $25 \%$ between Farms, and $18 \%$ and $30 \%$ for within-Farm variance. For $U U N(0)$ the fixed term Season was close to statistically significant (p $=0.067)$. The effects of FMType, Feed, and Calc were not statistically significant. Therefore, we simplified the model from Eq. (7.2) into Eq. (7.6):

$$
U U N_{i j k}(0)=\mu+T y_{q}+S e_{r}+F a_{i}+P u_{i j}+\epsilon_{i j k}
$$

with symbols according to Eq. (7.2) (Section 7.2.3). The effect of FMType was still taken into account since it represented the experimental design. Table 7.2 shows that the grand mean $U U N(0)$ was $4.27 \mathrm{~kg} \mathrm{~m}^{-3}$, with smaller values for $S F R$ and $G F$, and larger values for $A F$ and $S F C O$. The largest difference was $1.2 \mathrm{~kg} \mathrm{~m}^{-3}$ between $G F$ and $A F$. Table 7.2 shows that the $U U N(0)$ in the spring period $(S)$ was larger compared to the winter $(W)$, with a difference of about $0.6 \mathrm{~kg} \mathrm{~m}^{-3}$. The s.e. values for $U U N$ were large compared to the mean values, which confirms the large variance.

Table 7.1 shows that the between-Farm variance for $\mathrm{NH}_{3} S(0)$ was large. The within-Farm variance was even larger, and was about double the size of the betweenFarm variance. The effects of FMType, PREclean, and Season showed a statistically significant effect $(\mathrm{p}<0.05)$. However, the effect of FMType was just below the 0.05 level. The effects of Diet and Calc were not statistically significant. Therefore, we simplified the model from Eq. (7.3) into Eq. (7.7):

$$
N H_{3} S_{i j k}(0)=\mu+T y_{q}+C l_{p}+S e_{r}+F a_{i}+\epsilon_{i j k}
$$

with symbols according to Eq. (7.3) (Section 7.2.3). Table 7.2 shows that the $\mathrm{NH}_{3} S(0)$ was largest at $A F$ and smallest at $S F R$, with a difference of almost $5.0 \mathrm{~kg}$. $\mathrm{NH}_{3} S(0)$ was more or less halved by the PREclean treatment, which was fully caused by the $D_{p}(0)$ values (Snoek et al., 2016c). The table also show that the $\overline{N_{3} S(0)}$ in the spring period $(S)$ was larger compared to the winter $(W)$, with a difference of about $2.6 \mathrm{~kg}$. This was the combined cause of both larger $D_{p}(0)$ (Snoek et al., 2016c) and $U U N(0)$ values in the spring period. The s.e. values for $\mathrm{NH}_{3} S$ were large compared to the mean values, which confirms the large variance.

Finally, Table 7.3 shows the predicted means of the interaction between FMType and PREclean for $\mathrm{NH}_{3} \mathrm{~S}(0)$. This interaction was not statistically significant and was therefore not included in Eq. (7.7). The table, however, clarifies in more detail the intense-floor-cleaning effect (PREclean) per FMType. Without PREcleaning, the $\mathrm{NH}_{3} \mathrm{~S}(0)$ varies from $5.28 \mathrm{~kg}$ puddle ${ }^{-1}$ to $10.10 \mathrm{~kg}$ puddle ${ }^{-1}$, and with $P R E$ cleaning from $1.72 \mathrm{~kg}$ to $6.54 \mathrm{~kg}$. The difference was about $3.5 \mathrm{~kg}$ puddle ${ }^{-1}$ for each FMType. 


\subsubsection{Temperature and humidity}

Table 7.4 shows the mean and SD values per FMType and per Season for $T_{\text {liq }}, T_{\text {air }}$ and $R H$ for both the measured fresh puddles $(t=0 \mathrm{~s})$ and the random puddles $(t=\xi$ $\mathrm{s})$. For the fresh puddles, the puddle temperature $\left(T_{l i q}(0)\right)$ was higher than the air temperature $\left(T_{\text {air }}(0)\right)$. At $t=\xi$ s the puddle temperature $\left(T_{l i q}(\xi)\right)$ was comparable to the air temperature $\left(T_{\text {air }}(\xi)\right)$. For both $t=0 \mathrm{~s}$ and $t=\xi \mathrm{s}$ the puddle and air temperatures in winter $(W)$ were smaller than in spring $(S)$. The same was true for the relative humidity $(R H)$ but in the opposite direction, the $R H$ in $W$ was larger than in $S$. The mean values for $T_{\text {air }}(0)$ and $R H(0)$ were similar to $T_{a i r}(\xi)$ and $R H(\xi)$, which represent stable climatic circumstances during a measurement day.

Table 7.4: The number of samples (N) and the means with SD, per Type and Season. For $T_{l i q}(0), T_{a i r}(0), R H(0), T_{l i q}(\xi), T_{a i r}(\xi)$, and $R H(\xi)$.

\begin{tabular}{|c|c|c|c|c|c|c|c|c|c|c|c|c|}
\hline \multirow[b]{2}{*}{ Term } & \multicolumn{2}{|c|}{$T_{l i q}(0)\left[{ }^{\circ} \mathrm{C}\right]$} & \multicolumn{2}{|c|}{$\operatorname{Tair}(0)\left[{ }^{\circ} \mathrm{C}\right]$} & $R H(0)[\%]$ & \multicolumn{2}{|c|}{$T_{l i q}(\xi)\left[{ }^{\circ} \mathrm{C}\right]$} & \multicolumn{2}{|c|}{$\operatorname{Tair}(\xi)\left[{ }^{\circ} \mathrm{C}\right]$} & \multicolumn{3}{|c|}{$R H(\xi)[\%]$} \\
\hline & & mean $(\mathrm{sd})$ & & mean $(\mathrm{sd})$ & $\mathrm{N}$ mean $(\mathrm{sd})$ & & mean $(\mathrm{sd})$ & & mean $(\mathrm{sd})$ & & $\mathrm{J}$ mean & $(\mathrm{sd})$ \\
\hline$\mu$ & 295 & ) & 311 & ) & 30781 & 253 & 3.4) & 275 & 1.4) & 27 & 80.3 & ( \\
\hline \multicolumn{13}{|c|}{-Type- } \\
\hline & 68 & & 1 & & $1 \varepsilon$ & 51 & & 73 & & & $\begin{array}{ll}0 & 80.1\end{array}$ & $(7.8$ \\
\hline & 70 & & 2 & & 7 & 60 & & 60 & & 0 & & \\
\hline & 82 & & & & & 7 & & & & & & \\
\hline$C O$ & 75 & & 75 & 10 & 718 & 65 & 10 & 65 & & 65 & 581. & (10 \\
\hline \multicolumn{13}{|c|}{-Season- } \\
\hline$W$ & 158 & & 4 & & 16487 & 113 & & 125 & & 122 & 286. & $(4)^{\prime}$ \\
\hline a & 137 & 17 & 147 & 12.5 & 14375.6 & 140 & 12.1 & 150 & 12.6 & 150 & 074.8 & $(7$. \\
\hline
\end{tabular}

\subsubsection{Relationships between measured variables}

Figure 7.3 shows the relation between the $p H(0)$ and $U U N(0)$. The linear relation is described by $p H(0)=8.17+0.029 \cdot U U N(0)$, which is statistically significant. The p-value of the linear term is 0.03 , but the $R_{a d j}^{2}$ is low at $<0.03$ and the relation is approximately a horizontal line. The regression line starts at a $p H$ of 8.2 and increases to 8.5 , with $95 \%$ confidence intervals of 7.7 to 8.9 .

Figures $7.4 \mathrm{a}, 7.5 \mathrm{a}$ and $7.5 \mathrm{~b}$ show the relations of $U U N(0), p H(0)$ and $p H(\xi)$ with $T_{a i r}$. There was a statistically significant linear increase $(p=<0.001)$ for all three variables. When $T_{\text {air }}$ increased, however, the $R_{a d j}^{2}$ was low at $<0.09,<0.04$ and $<0.08$, respectively. The total increase of the linear regression line for a $T_{\text {air }}$ of $0{ }^{\circ} \mathrm{C}$ to $20^{\circ} \mathrm{C}$ was small for all three variables, whereas the data distribution was wide:

- For $U U N(0)$ the linear relation was described by $U U N(0)=2.93+0.14 \cdot T_{a i r}(0)$. The regression started at a $U U N$ of $2.9 \mathrm{~kg} \mathrm{~m}^{-3}$ and increased to $5.7 \mathrm{~kg} \mathrm{~m}^{-3}$, with $95 \%$ confidence intervals of $0.7 \mathrm{~kg} \mathrm{~m}^{-3}$ to $7.6 \mathrm{~kg} \mathrm{~m}^{-3}$. 
- For $p H(0)$ the linear relation was described by $p H(0)=8.19+0.015 \cdot T_{a i r}(0)$. The regression started at a $p H$ of 8.2 and increased to 8.5, with $95 \%$ confidence intervals of 7.7 to 8.9 .

- For $p H(\xi)$ the linear relation was described by $p H(\xi)=8.82+0.018 \cdot T_{a i r}(\xi)$. The regression started at a $p H$ of 8.8 and increased to 9.2 , with $95 \%$ confidence intervals of 8.5 to 9.5 .

Comparable results were shown for the relations of $U U N(0), p H(0)$ and $p H(\xi)$ with $R H$. The regression line, however, decreased at increasing $R H$ values, since the $T_{\text {air }}$ and $R H$ were negatively correlated.

Figure $7.4 \mathrm{~b}$ shows the relation of $M U N$ with $T_{\text {air }}$. There was a statistically significant linear decrease $(p=0.01)$ of $M U N=26.91-0.39 \cdot \overline{T_{a i r}(0)}$, with an $R_{a d j}^{2}$ of 0.18 . The figure shows that the $M U N$ decreased with an increasing $T_{a i r}$. However, the confidence intervals were wide indicating a low strength. A horizontal line can be drawn between them.

Figures 7.6a and 7.6b show the relations between the $T_{l i q}$ and $T_{\text {air }}$ at $t=0 \mathrm{~s}$ and $t=\xi \mathrm{s}$, respectively. At $t=\xi \mathrm{s}$, there is a strong statistical significant linear regression $(p=<0.001)$ of $T_{l i q}(\xi)=3.14+0.74 \cdot T_{a i r}(\xi)$, with an $R_{a d j}^{2}$ of 0.83 . At $t=0 \mathrm{~s}$, the linear regression is still statistically significant $(p=<0.001) T_{l i q}(0)=11.06+0.49 \cdot T_{a i r}(0)$, but the $R_{a d j}^{2}$ is lower $(0.28)$ and the $T_{l i q}(0)$ data is more widely spread compared to $t=\xi$ s data. The relations of $U U N(0), p H(0)$ and $p H(\xi)$ with $T_{l i q}(0)$ are not statistically significantly linear.

Figure 7.7 shows the relationship between $\overline{U U N(0)}$ and the $M U N$ and Fig. 7.8 shows the relationship between $\overline{p H(\xi)}$ and $\overline{p H(0)}$, both per Farm and per Season. Both linear relations were not statistically significant and were therefore left out of the figures. One data point in Fig. 7.8 in the upper right corner $(+)$ was excluded from the regression analysis. Since the point showed an extreme high leverage of 0.3134 compared to the mean leverage of 0.0690, and an extreme high Cook's distance of 0.615 compared to the mean Cook's distance of 0.0577. Both figures show that the data were scattered.

Similar plots were made and studied for the relations between the remaining variable combinations. There were no linear relations of $U U N(0), p H(0), T_{l i q}, T_{a i r}$, or $R H$ with either $A_{p}(0)$ or $D_{p}(0)$.

\subsection{Discussion}

\subsubsection{UUN and MUN}

Our $U U N$ data agreed well with values found in literature. Monteny et al. (2002) reported Urinary Urea concentrations $(U U N)$ values of fresh urine ranging from 


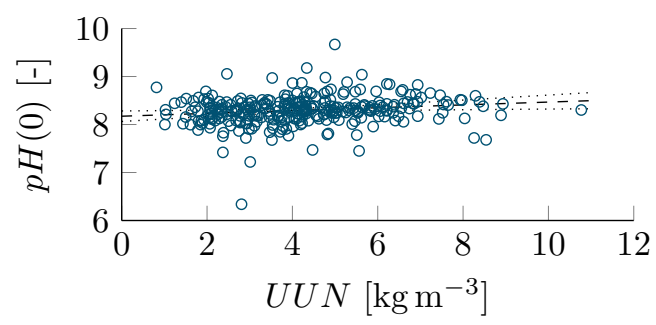

Figure 7.3: $p H(0)$ vs $U U N(0)(\circ)$, with regression: $p H(0)=8.17+0.029 \cdot U U N(0)$ $(---)$ and $95 \%$ confidence intervals (…...).

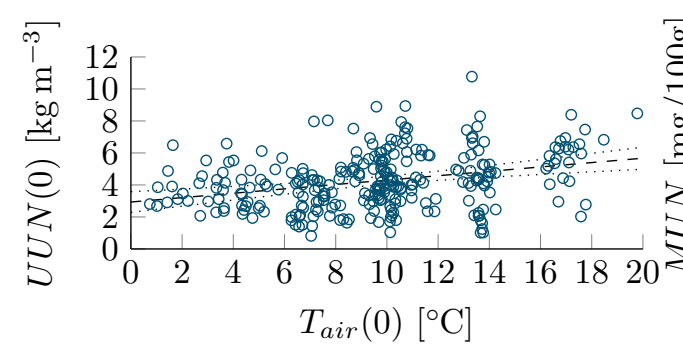

(a) $U U N(0)=2.93+0.14 \cdot T_{\text {air }}(0)$.

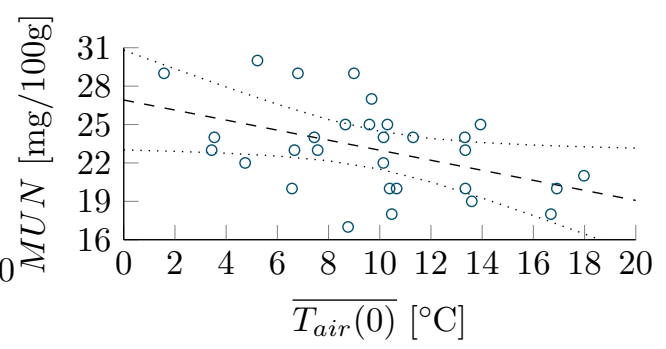

(b) $M U N=26.91-0.39 \cdot \overline{T_{a i r}(0)}$.

Figure 7.4: $U U N(0)$ vs $T_{\text {air }}(0)$ (a) and $M U N$ vs $\overline{T_{a i r}(0)}(\mathrm{b})(\circ)$, with regression (- - - ) and $95 \%$ confidence intervals $(\cdots \cdots)$.

$2.7 \mathrm{~kg} \mathrm{~m}^{-3}$ to $12.1 \mathrm{~kg} \mathrm{~m}^{-3}$ for pooled samples of 15 cows. The $U U N$ was obtained for varying feed characteristics based on the grass and maize content in the roughage and the Rumen-Degradable Protein balance (RDP). In the same study, they reported modelpredicted values that were slightly lower and ranged from $1.7 \mathrm{~kg} \mathrm{~m}^{-3}$ to $11.6 \mathrm{~kg} \mathrm{~m}^{-3}$. In an accompanying paper related to Monteny et al. (2002), it was explained that the urinary $\mathrm{N}$ increased when the RDP of the diet increased as well as when the corn part in the roughage increased (de Boer et al., 2002). Burgos et al. (2005) reported $U U N$ values of fresh urine of $4.27 \mathrm{~kg} \mathrm{~m}^{-3}(\mathrm{SD}=1.77)$ and $3.75 \mathrm{~kg} \mathrm{~m}^{-3}(\mathrm{SD}=2.76)$ for two commercial dairy farms, based on 33 and 15 cows, respectively. In another study, Burgos et al. (2007) reported $U U N$ values of fresh urine that ranged from $3.55 \mathrm{~kg} \mathrm{~m}^{-3}$ to $9.03 \mathrm{~kg} \mathrm{~m}^{-3}$ for three lactation stages and four dietary crude protein (CP) levels. These stages and levels were created by adding urea to the feed of the cows. The $U U N$ was obtained in a split-plot Latin square with 12 cows. This arrangement resulted in only one cow per randomly assigned unique treatment. The large range resulted from the four CP levels, i. e. larger CP resulted in larger $U U N$ values. The lactation stages affected $U U N$ to a more limited extent, resulting in a 

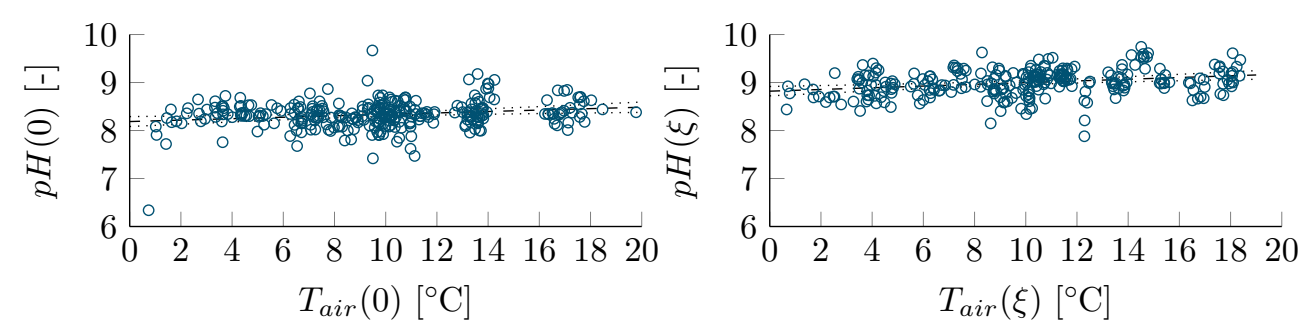

(a) $t=0 \mathrm{~s}, p H(0)=8.19+0.015 \cdot T_{\text {air }}(0)$.

(b) $t=\xi \mathrm{s}, p H(\xi)=8.82+0.018 \cdot T_{\text {air }}(\xi)$.

Figure 7.5: $p H$ vs $T_{\text {air }}(\circ)$ at $t=0 \mathrm{~s}$ (a) and $t=\xi \mathrm{s}(\mathrm{b})$, with regression (-- - ) and $95 \%$ confidence intervals $(\cdots \cdots \cdot)$.
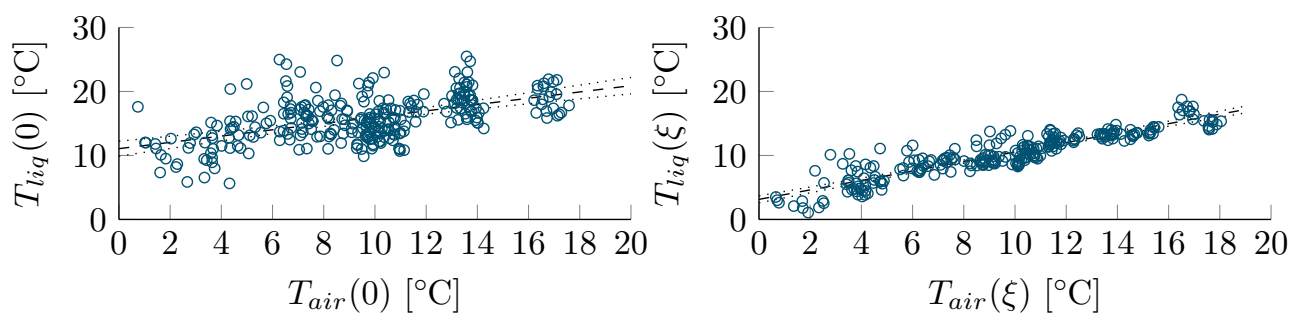

(a) $t=0 \mathrm{~s}, T_{l i q}(0)=11.06+0.49 \cdot T_{\text {air }}(0)$.

(b) $t=\xi \mathrm{s}, T_{l i q}(\xi)=3.14+0.74 \cdot T_{a i r}(\xi)$.

Figure 7.6: $T_{l i q}$ vs $T_{\text {air }}(\circ)$ at $t=0 \mathrm{~s}(\mathrm{a})$ and $t=\xi \mathrm{s}(\mathrm{b})$, with regression (--- ) and $95 \%$ confidence intervals $(\cdots \cdots . .$.$) .$

range from $5.88 \mathrm{~kg} \mathrm{~m}^{-3}$ to $6.62 \mathrm{~kg} \mathrm{~m}^{-3}$. Finally, van Duinkerken et al. (2011) reported $(U U N)$ values of fresh urine that increased from $2.5 \mathrm{~kg} \mathrm{~m}^{-3}$ to $7.9 \mathrm{~kg} \mathrm{~m}^{-3}$ for pooled samples of at least 12 cows. The $U U N$ was obtained from three increasing levels of Milk Urea Nitrogen $(M U N)$ that where induced by combining feed and pasture management, both of which increased the RDP. The $U U N(0)$ values reported in our study showed a comparable, yet slightly smaller range of $2.09 \mathrm{~kg} \mathrm{~m}^{-3}$ to $6.79 \mathrm{~kg} \mathrm{~m}^{-3}$ at individual Farm level (Table 7.5). Our data range was close to Burgos et al. (2005) but slightly lower compared to Monteny et al. (2002), Burgos et al. (2007) and van Duinkerken et al. (2011). The $U U N$ range-shift between the studies may be attributed to the measurement time during the day. Van Duinkerken et al. (2011) sampled urine twice, just before milking in the morning and just before milking in the afternoon. The morning samples had a higher $U U N$ value than the afternoon ones for all feed treatments. In addition to morning and afternoon measurement moments, Burgos et al. (2007) sampled urine around noon and midnight. They showed that the morning and afternoon samples were approximately equal, whereas the noon samples were lower, and the midnight samples were higher. The results of both our study and Burgos 


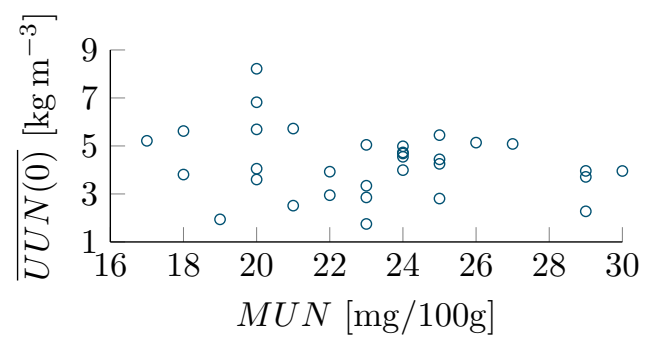

Figure 7.7: $\overline{U U N(0)}$ vs $M U N(\circ)$.

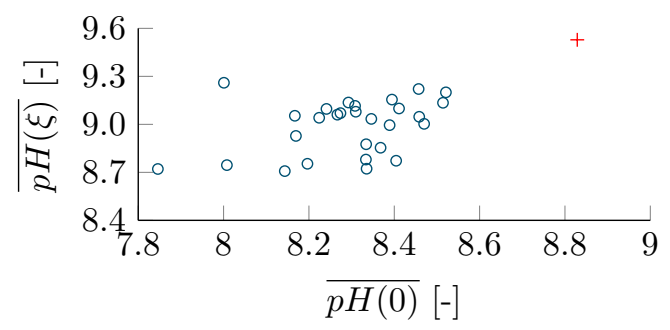

Figure 7.8: $\overline{p H(\xi)}$ vs $\overline{p H(0)}(\circ)$. The '+' indicates an outlier with high leverage, which was excluded from the regression analysis.

et al. (2005) were obtained during the morning after milking and in our study closer to noon.

In our REML analyses, none of the experimental factors showed a statistically significant difference for $U U N$. For FMType we did not expect different $U U N$ values. However, it is striking that the $U U N$ at the $S F C O$ farms is among the largest, while the Cows \& Opportunities project aimed at efficient use of nutrients at farm level (Oenema et al., 2001; Aarts et al., 2015). Consequently, low $U U N$ values were expected at these SFCO farms. Based on the literature we did expect effects for Seasons or Diets. Our Diet information, however, was limited. This may may explain why there was no significant difference for this factor. At Season level the difference was considerable, approximately $0.6 \mathrm{~kg} \mathrm{~m}^{-3}$. Our $U U N$ data, however, showed a large variation, with the result that this seasonal difference was not large enough to be distinguished from 0 .

The $U U N$ reference value currently used by the Technical Advisory Committee (Tac-Rav) in the Tac-Rav model is $5.0 \mathrm{~kg} \mathrm{~m}^{-3}$. The reference is within the range of all the above-mentioned studies and within the range of our measurement data. The grand mean of the current study, however, was lower, with a value of $4.27 \mathrm{~kg} \mathrm{~m}^{-3}$. As explained above, this lower $U U N$ may be attributed, to some extent, to the 
measurement moment during the day.

The $M U N$ is obtained from the milk tank and represents a mean value of a farm from one to three days before the measurement day. In other words, the $M U N$ does not represent individual cows, like the $U U N$, but a farm mean. Both Burgos et al. (2007) and van Duinkerken et al. (2011) showed a strong relation with an increasing trend between the $U U N$ and $M U N$. A strong linear relation, however, was not found in our study and also not in the study of Burgos et al. (2005). Figure 7.7 in our study and Figure 3 in Burgos et al. (2005) show a wide spread of data with no statistically significant linear relation between $M U N$ and $U U N$. There was, however, a main difference between the two pairs of studies. Both Burgos et al. (2007) and van Duinkerken et al. (2011) performed a controlled diet experiment, whereas both our study and Burgos et al. (2005) performed measurements in commercial dairy cow house with no predefined or controlled diets. The controlled diet experiments were done for only a short period of time and several diets contained high levels of proteins, which may not be the case in commercial dairy cow houses. To summarise, the reported relation between $M U N$ and $U U N$ with strong diet contrasts may not be visible in practical studies with less variation between diets. Other factors that affect $M U N$ and $U U N$ are apparently more dominant.

\subsection{2 $\mathrm{pH}$}

Several studies reported $p H$ values in fresh urine, ranging from 7.9 to 8.6 (Monteny et al., 2002), at $8.11(\mathrm{SD}=0.25)$ and at $7.92(\mathrm{SD}=0.31)$ (Burgos et al., 2005) and ranging from 8.2 to 8.4 (van Duinkerken et al., 2011). Possible causes for the $p H$ differences were not reported. Monteny et al. (2002) discussed that $p H$ measurement of dairy cow urine is needed for $\mathrm{NH}_{3}$ emission modelling since the estimated $\mathrm{NH}_{3}$ is very sensitive to $p H$. In a previous study, $p H(0)$ values of 8.1 to 8.4 measured at three farms were reported (Snoek et al., 2016b) both in summer and winter. The $p H(0)$ differences were not statistically significant in that study. The $p H(0)$ values reported in the current study showed a comparable range of 8.1 to 8.5 at individual Farm level (Table 7.5) only Burgos et al. (2005) showed slightly lower values. None of the experimental factors showed statistically significant differences in our study and the variability was low with $\mathrm{CV}$ values of $<2.5 \%$ between means of measurement days. The estimated grand mean amounted to 8.3 with a low s.e. due to the small variation. In conclusion, all these reported $p H(0)$ data indicate that the initial $p H$ after urination by a cow is around 8.3.

Monteny et al. (2002), Burgos et al. (2005) and van Duinkerken et al. (2011) did not report $p H$ values of older puddles. In Snoek et al. (2016b) $p H$ values were measured for $4 \mathrm{~h}$. They showed that the $p H$ curve stabilised after about $1 \mathrm{~h}$, and the $p H$ range at that time was 8.8 to 9.3 at the individual farm level and 8.9 to 9.2 between summer 
and winter. At $t=4 \mathrm{~h}$ both ranges shifted up with a $p H$ of 0.1 . The differences at farm and season level were statistically significant but not between each individual farm. The $p H$ values of random puddles with random age $(p H(\xi))$ reported in this study showed a comparable range of 8.8 to 9.2 at individual Farm level (Table 7.5). Grouped by FMType or Season, these puddles showed no statistically significant differences. However, they did show a statistically significant difference for Diet, but the absolute difference was small with a $p H$ of only 0.1 . The estimated grand mean $p H(\xi)$ of 9.0 showed a low s.e. In conclusion, all these reported $p H(\xi)$ data indicate that the $p H$ of a random puddle in a cow house is around 9.0. Furthermore, the floor material asphalt did not affect the $p H$ compared to concrete.

The $p H$ reference value currently used by the Tac-Rav in the Tac-Rav model is 9.4 for a concrete slatted floor. The Tac-Rav model uses a fixed $p H$ value that is the same for each individual puddle during the whole puddle simulation period of a full dairy cow house in the $\mathrm{NH}_{3}$ emission model (Monteny et al., 1998). The $p H$, however, is not fixed at 9.4. Our study shows that the initial $p H$ of 8.3 increases to a $p H$ of 9.0 for a random puddle, which is still lower than the value used in the Tac-Rav model. In addition, Snoek et al. (2016b) showed comparable results as discussed above, and they showed a curve to represent the $p H$ increase in a fresh dairy cow urine puddle. Based on this study and our previous study (Snoek et al., 2016b), we recommend using the proposed $p H$ curve in $\mathrm{NH}_{3}$ emission modelling. To further investigate the effect of a $p H$ curve, an updated Tac-Rav model needs to be built and validated.

\subsubsection{Temperature and humidity}

The $T_{\text {air }}$ reference value currently used by the Tac-Rav in the Tac-Rav model is $10^{\circ} \mathrm{C}$. This temperature is comparable to the measured $T_{\text {air }}$ in this study. An air humidity value is not included in the model. The Tac-Rav model uses a fixed $T_{\text {air }}$ value that is the same for each individual puddle within the simulation of a full dairy cow house in the $\mathrm{NH}_{3}$ emission model (Monteny et al., 1998). The $T_{a i r}$, however, is not fixed at $10^{\circ} \mathrm{C}$. There will be daily and seasonal temperature patterns, but based on the result of the current study, we cannot draw conclusions about what these patterns should be.

\subsubsection{Relationships between measured variables}

The relation of $U U N(0)$ vs. $T_{a i r}$ was statistically significant with a low $R_{a d j}^{2}$ and wide spread of the data compared to the total increase of the linear regression. In contrast to $U U N$, the $M U N$ decreased at larger $T_{\text {air }}$. When the temperature rises, probably some factors, such as the feed and water intake, the metabolism, the activity, and the milk production of the cows will change. These changes may cause a change in both the $U U N$ and $M U N$. It appears that milk starts to contain a smaller concentration of urea at larger temperatures compared to urine. It can also be that the amount 
of milk increases faster than the amount of urea compared to urine. Based on the current study, however, we do not know the amounts in $\mathrm{kg}$, since we did not measure the volume of milk or urine per cow. Additionally, there was no clear relation between $U U N$ and $M U N$ (Section 7.4.1).

\subsubsection{Ammonia emission potential}

The calculated $\mathrm{NH}_{3} \mathrm{~S}(0)$ represents the potential $\mathrm{NH}_{3}$ source. If all $U U N$ will convert to $\mathrm{NH}_{3}$ and emit, this source represents the maximum $\mathrm{NH}_{3}$ emission from a urine puddle. The relation between $U U N$ and $\mathrm{NH}_{3}$ emission in cow houses is known to be strong (Monteny et al., 2002; Burgos et al., 2010).

The $\mathrm{NH}_{3} \mathrm{~S}(0)$ reference value from the Tac-Rav model is $2.33 \times 10^{-3} \mathrm{~kg}_{\text {puddle }}{ }^{-1}$ for a concrete slatted floor (Eq. (7.8)). This value is based on the currently used Tac-Rav reference puddle values for area $\left(A_{p} ; 0.8 \mathrm{~m}^{2}\right)$, depth $\left(D_{p} ; 0.48 \mathrm{~mm}\right)$ and $U U N$ $\left(5.0 \mathrm{~kg} \mathrm{~m}^{-3}\right)$. With a urination frequency of $10 \# \mathrm{cow}^{-1} \mathrm{~d}^{-1}$ (Monteny et al., 1998) and $365 \mathrm{~d} \mathrm{yr}^{-1}$, the potential $\mathrm{NH}_{3} S$ results in an $\mathrm{NH}_{3}$ emission of $8.5 \mathrm{~kg} \mathrm{cow}^{-1} \mathrm{yr}^{-1}$ (Eq. (7.9)):

$$
\begin{aligned}
5.0 \cdot 0.8 \cdot 0.48 \cdot 10^{-3} \cdot \frac{17}{14} & =2.33 \cdot 10^{-3} \\
2.33 \cdot 10^{-3} \cdot 10 \cdot 365 & =8.5
\end{aligned}
$$

with $\frac{17}{14}$ to convert from $\mathrm{NH}_{3}-\mathrm{N}$ to $\mathrm{NH}_{3}$ (Monteny et al., 1998).

In the current study the $\mathrm{NH}_{3} S(0)$ was $5.28 \times 10^{-3} \mathrm{~kg}$ puddle ${ }^{-1}$ or $19.3 \mathrm{~kg} \mathrm{cow}^{-1} \mathrm{yr}^{-1}$ at a slatted floor $(S F R)$ without $P R E$ clean treatment. This value is $226 \%$ larger than the Tac-Rav reference value. The $S F R$ with PREclean treatment had an $\mathrm{NH}_{3} S(0)$ of $1.72 \times 10^{-3} \mathrm{~kg}$ puddle ${ }^{-1}$ or $6.3 \mathrm{~kg} \mathrm{cow}^{-1} \mathrm{yr}^{-1}$. This value is lower than the reference and the lowest within this study. The largest values originated from the V-shaped asphalt floor $(A F)$ without PREclean treatment. The $\mathrm{NH}_{3} S(0)$ was $10.10 \times 10^{-3} \mathrm{~kg}$ or $36.9 \mathrm{~kg} \mathrm{cow}^{-1} \mathrm{yr}^{-1}$. This was much larger than the Tac-Rav reference value. These large differences between our study and the Tac-Rav reference were mainly related to the $D_{p}$ values (Snoek et al., 2016c). Furthermore, the PREclean treatment has a substantial effect (Snoek et al., 2016c). In conclusion, the $\mathrm{NH}_{3}$ emission from the floor in a dairy cow house may be much larger than is currently estimated and assumed by the Tac-Rav. Since we did not measure $\mathrm{NH}_{3}$ emission, we cannot validate these emission estimates. 


\subsection{Conclusions}

The objective of this research was to investigate the Urinary Urea Nitrogen concentration $(U U N)$, the related potential $\mathrm{NH}_{3}$ source size $\left(\mathrm{NH}_{3} S(0)\right)$, the initial $p H(p H(0))$, the $p H$ for a random puddle at a random moment $(p H(\xi))$, the puddle and the air temperature $\left(T_{l i q}, T_{a i r}\right)$, and the relations between them in commercial dairy cow houses.

Estimated overall mean values were the following:

- $\overline{U U N(0)}$ was $4.27 \mathrm{~kg} \mathrm{~m}^{-3}$ with a large variation (CV $=18.1 \%$ to $\left.29.8 \%\right)$;

- the resulting $\overline{\mathrm{NH}_{3} S(0)}$ was $5.3 \times 10^{-3} \mathrm{~kg}$ with a large variation (CV $=20.1 \%$ to $43.3 \%$ );

- $\overline{p H(0)}$ was 8.3 with a small variation $(\mathrm{CV}=<3.0 \%)$;

- $\overline{p H(\xi)}$ was 9.0 with a small variation $(\mathrm{CV}=<2.5 \%)$

The measured $U U N(0)$ agreed well with the known values based on feed studies, but it was slightly lower than the reference used by the Technical Advisory Committee (Tac-Rav). UUN values from commercial dairy cow houses varied stronlgy, both within one farm as well as between farms. The measured $p H(0)$ also agreed well with the known values based on feed studies, but currently an initial $p H$ is not used by the Tac-Rav. The measured $p H(\xi)$ agreed well with another study about long-term $p H$ measurements in commercial dairy cow houses, but it was slightly lower than the reference used by the Tac-Rav. $p H$ data variance was small and stable both within one farm and between farms. Finally, the estimated $\mathrm{NH}_{3}$ potential $\left(\mathrm{NH}_{3} \mathrm{~S}\right)$ showed a huge range and was considerably larger than the Tac-Rav reference values.

The factors FMType, PREclean and Season had a statistically significant effect on the calculated $\mathrm{NH}_{3} S(0)$. This effect was the combined result of the $U U N, A_{p}$ and $D_{p}$ values, of which $D_{p}$ had the largest range. The Diet factor showed a statistically significant effect on $p H(\xi)$, but the $p H$ difference between the two levels was only 0.1 . For $U U N(0)$ and $p H(0)$, none of the factors showed a statistically significant effect.

The $\overline{T_{l i q}(0)}$ was $15.6{ }^{\circ} \mathrm{C}$ and $\overline{T_{l i q}(\xi)}$ was $10.3^{\circ} \mathrm{C}$. For fresh puddles the $\overline{T_{a i r}(0)}$ with a value of $9.7^{\circ} \mathrm{C}$ was about $5^{\circ} \mathrm{C}$ lower than the $\overline{T_{l i q}(0)}$. For random puddles the $\overline{T_{a i r}(\xi)}$ with a value of $10.0^{\circ} \mathrm{C}$ was comparable to the $\overline{T_{l i q}(\xi)}$.

There were no clear relations between $p H$ and $U U N$ or $T_{\text {air }}$ or between $U U N$ and $M U N$.

With the obtained values and distributions for $U U N, p H, T_{a i r}$, and $A_{p} \& D_{p}$ (Snoek et al., 2016c), $\mathrm{NH}_{3}$ emission estimations can be performed with the Tac-Rav model. These estimates can then be compared to the current Tac-Rav values to identify potential model improvements. Based on our results, we recommend including 
$U U N(0)$ and $D_{p}(0)$ (Snoek et al., 2016c) measurements in the currently used protocol for measuring individual full scale dairy cow houses (Ogink et al., 2013). In addition, we recommend determining the $A_{p}$ at varying floor designs in practice since the FMType had a significant effect on the $A_{p}$ value (Snoek et al., 2016c). However, it will not be necessary to include $A_{p}$ in the full-scale-protocol. Finally, we recommend further investigating the relation between $U U N, M U N$ and $p H$, and their relation with feed management and $\mathrm{NH}_{3}$ emission from commercial dairy cow houses to improve our understanding of the related processes and effects in practice.

\subsection{Acknowledgements}

The authors would like to thank the 16 dairy farmers involved in the study for their hospitality, cooperation during measurements, and for their contributions to the discussions on this research. the authors would also like to thank W. Bin, S. Blaauw, S. Bomers, A. van Dueren den Hollander, and R. Vernooij for their assistance during the measurements. Language editing of a near-final draft of the paper was provided by P. Griffith.

\subsection{Supplementary Material}

see Table 7.5. 
Table 7.5: The number of fresh urine puddles (N), the mean (SD) per Farm, and the p-value to test equality of means. For variables $p H(0), p H(\xi), U U N(0), N_{3} S(0)_{o f f}{ }^{\dagger}$, and $\mathrm{NH}_{3} \mathrm{~S}(0)_{\text {on }}^{\dagger}$.

\begin{tabular}{|c|c|c|c|c|c|c|c|c|c|c|}
\hline \multirow[b]{2}{*}{ Farm } & \multicolumn{2}{|r|}{$p H(0)[-]$} & \multicolumn{2}{|r|}{$p H(\xi)[-]$} & \multicolumn{2}{|c|}{$U U N(0)\left[\mathrm{kg} \mathrm{m}^{-3}\right]$} & \multicolumn{2}{|c|}{$\mathrm{NH}_{3} S(0)_{o f f}^{\dagger}[\mathrm{kg}]$} & \multicolumn{2}{|c|}{$\mathrm{NH}_{3} S(0)_{\text {on }}^{\dagger}[\mathrm{kg}]$} \\
\hline & $\mathrm{N}$ & Mean (SD) & $\mathrm{N}$ & Mean (SD) & $\mathrm{N}$ & Mean (SD) & $\mathrm{N}^{\ddagger}$ & $\begin{array}{l}\text { Mean (SD) } \\
\cdot 10^{-3}\end{array}$ & $\mathrm{~N}^{\ddagger}$ & $\begin{array}{l}\text { Mean (SD) } \\
\cdot 10^{-3}\end{array}$ \\
\hline$F 1$ & 25 & $8.2(0.36)$ & 11 & $9.2(0.21)$ & 16 & $4.33(1.82)$ & 2 & $5.26(0.57)$ & 1 & $3.67(-)$ \\
\hline$F 2$ & 21 & $8.5(0.21)$ & 10 & $9.1(0.29)$ & 20 & $6.79(2.46)$ & 2 & $12.70(3.89)$ & 2 & $5.25(2.96)$ \\
\hline$F 3$ & 23 & $8.4(0.18)$ & 21 & $9.1(0.20)$ & 18 & $3.53(1.53)$ & 2 & $6.62(4.86)$ & 2 & $1.86(0.71)$ \\
\hline$F 4$ & 20 & $8.3(0.23)$ & 10 & $8.8(0.28)$ & 18 & $2.09(0.70)$ & 2 & $3.14(1.90)$ & 2 & $2.46(2.38)$ \\
\hline$F 5$ & 19 & $8.3(0.22)$ & 15 & $9.1(0.19)$ & 19 & $4.93(1.85)$ & 2 & $5.26(0.56)$ & 2 & $3.39(0.85)$ \\
\hline$F 6$ & 20 & $8.3(0.15)$ & 20 & $8.9(0.23)$ & 19 & $2.41(0.75)$ & 2 & $5.57(1.77)$ & 1 & $1.94(-)$ \\
\hline$F 7$ & 20 & $8.3(0.21)$ & 20 & $9.0(0.21)$ & 20 & $3.10(0.83)$ & 2 & $3.39(0.61)$ & 2 & $1.49(0.62)$ \\
\hline$F 8$ & 21 & $8.4(0.21)$ & 20 & $8.9(0.30)$ & 19 & $4.64(1.15)$ & 2 & $3.13(0.40)$ & 2 & $2.32(1.41)$ \\
\hline$F 9$ & 20 & $8.2(0.43)$ & 20 & $9.0(0.16)$ & 20 & $4.52(1.53)$ & 2 & $7.16(1.92)$ & 2 & $4.33(1.87)$ \\
\hline$F 10$ & 21 & $8.4(0.20)$ & 20 & $9.0(0.24)$ & 19 & $4.85(1.50)$ & 2 & $12.00(8.21)$ & 2 & $7.81(5.65)$ \\
\hline$F 11$ & 20 & $8.1(0.43)$ & 20 & $8.9(0.34)$ & 14 & $6.07(2.44)$ & 2 & $16.80(12.2)$ & 2 & $6.20(3.63)$ \\
\hline$F 12$ & 21 & $8.2(0.21)$ & 10 & $8.9(0.19)$ & 19 & $3.03(1.21)$ & 2 & $4.50(1.23)$ & 2 & $1.85(1.40)$ \\
\hline$F 13$ & 20 & $8.5(0.41)$ & 20 & $9.1(0.46)$ & 20 & $4.73(0.98)$ & 2 & $7.99(0.31)$ & 2 & $4.54(3.39)$ \\
\hline$F 14$ & 20 & $8.4(0.09)$ & 20 & $9.1(0.10)$ & 20 & $5.01(1.56)$ & 2 & $10.20(2.44)$ & 2 & $5.75(1.79)$ \\
\hline$F 15$ & 20 & $8.3(0.16)$ & 20 & $8.9(0.24)$ & 20 & $4.32(1.11)$ & 2 & $4.54(3.90)$ & 2 & $2.96(3.08)$ \\
\hline$F 16$ & 22 & $8.2(0.44)$ & 20 & $8.9(0.28)$ & 19 & $3.76(1.60)$ & 2 & $5.01(0.22)$ & 2 & $1.54(0.80)$ \\
\hline p-value & & $<0.001$ & & $<0.001$ & & $<0.001$ & & 0.001 & & 0.243 \\
\hline
\end{tabular}

$\dagger \mathrm{NH}_{3} \mathrm{~S}(0)$ was calculated according to Eq. (7.1), without PREclean (off) or with (on).

$\ddagger \mathrm{N}$ is the number of Seasons.

$\$$ p-value of REML F-statistic 



\section{CHAPTER 8}

\section{General Discussion}

Dennis Snoek

\subsection{Introduction}

In the late nineties, the EU National Emission Ceiling Directive (NECD), among others, set $\mathrm{NH}_{3}$ emission ceilings EU wide and for each member country, for the year 2010. From 1990 to 2010 the ammonia $\left(\mathrm{NH}_{3}\right)$ emission decreased EU wide with $28 \%$, and the set targets were achieved by each member country (EEA, 2012). However, the European Commission (EC) proposed a new European Clean Air Programme with an updated NECD for the year 2020 and beyond (EEA, 2015). In addition, the EC reported that agriculture still dominates the $\mathrm{NH}_{3}$ emission, and that compared to other pollutants the $\mathrm{NH}_{3}$ emissions did not decrease to the same extent since 1990. In The Netherlands, the $\mathrm{NH}_{3}$ emission from dairy cow houses is one of the most important contributors (Velthof et al., 2012).

Over the past 25 years a lot of research has been conducted to understand and model the $\mathrm{NH}_{3}$ emission from dairy cow houses (Elzing \& Monteny, 1997), and to measure it (Mosquera et al., 2005b). In addition, reduction measures were identified (e.g. Monteny \& Erisman, 1998) and further developed for implementation in commercial dairy cow houses, which is regulated by the Ministry of Infrastructure and Environment, supported by a Technical Advisory Committee (Tac-Rav). The Tac-Rav, among other sources of information, makes use of the $\mathrm{NH}_{3}$ emission model developed by Monteny et al. (1998) to pre-assess new cow housing systems. However, still some important aspects remain unclear. For example, the values for the input variables of this Tac-Rav model and the relations with the $\mathrm{NH}_{3}$ emission are limitedly known. Moreover, these 
input variables are not measured in practice, since they are not included in the currently applied measurement protocol (Ogink et al., 2013). Therefore, the introduction and (pre)-assessment of new reduction methods is complicated and subject to a high level of uncertainty. Besides, the functioning of applied reduction measures in commercial dairy cow houses is limitedly known.

The overall objective of this $\mathrm{PhD}$ thesis was

\section{Refining a model-based assessment strategy to estimate the ammonia emission from floors in dairy cow houses}

The overall objective was split up in four sub-objectives that were addressed in chapters 2 to 7 :

1. Identify the most important input variables and process parameters in current available mechanistic $\mathrm{NH}_{3}$ emission models and theory (Chapter 2).

2. Explore, identify, develop, and improve sensors and measurement methods for these most important input variables to measure them in commercial dairy cow houses (Chapters 3, 4 and 5).

3. Assess the values and interactions of the identified input variables for fresh urine puddles in commercial dairy cow houses (Chapters 3, 6 and 7).

4. Study the effect of these input variable values of fresh urine puddles on the estimated $\mathrm{NH}_{3}$ emission (Chapter 7 ).

In Chapter 2 it was concluded that five urine puddle related variables explained at least $71 \%$ of the variation in the model estimated $\mathrm{NH}_{3}$ emission from the floor in dairy cow houses. These variables are, in order of importance, puddle $p H$, depth $\left(D_{p}\right)$, urinary urea nitrogen concentration $(U U N)$, surface area $\left(A_{p}\right)$, and temperature $\left(T_{l i q}\right)$. Furthermore, for each input variable in the model the available data was scarce, and it was therefore recommended to measure the five most important variables in practice. The main cause for the data scarcity was the lack of accurate sensors and the complex circumstances to measure the selected five variables in commercial cow houses.

In Chapter 3 both a $p H$ sensor and a temperature sensor were selected, tested and applied to measure the $\mathrm{pH}$ and temperature of 26 fresh dairy cow urine puddles in commercial dairy cow houses. From the $p H$ series a mean $p H$ curve was distinguished that had an initial $p H$ of 8.3 and increased to 9.2 after 4 h. Ninety percent of this $p H$ increase was realised within the first hour.

In Chapter 4 A so-called XY-table with an ultrasonic distance sensor attached to it was tested as method to obtain the $D_{p}$ values from urine puddles in commercial 
dairy cow houses. The depth of water puddles was successfully measured in various experimental setups and the ultrasonic method reached a measurement uncertainty of $<0.1 \mathrm{~mm}$.

In a preliminary experiment, a thermal IR camera was mounted on a movable trolley and was able to collect IR images of fresh urine puddles to determine $A_{p}$ (Snoek et al., 2014a). In Chapter 5 an IR model was developed and tested to obtain the $A_{p}$ from an IR image. The $A_{p}$ was underestimated by $2.53 \%$ compared to the reference method for which can be compensated, and the resulting measurement uncertainty was $<0.1 \mathrm{~m}^{2}$.

The selected sensors and developed methods were combined and a protocol was described to measure the $p H, D_{p}, U U N, A_{p}$ and $T_{l i q}$ of fresh, random and manually created urine puddles in commercial dairy cow houses (Chapter 6 ). In a factorial experimental setup based on four floor-management types (FMType), with experimental factors Season and PREclean treatment. PREclean was only applied to the $D_{p}$ measurement and represented the intense-floor-cleaning before urine puddle creation, which was compared with those created under normal floor conditions with on-farm manure scraping. Two explanatory factors were distinguished that were not controlled at farm level and were not equally spread among the FMTypes, being Diet and Calc.

In Chapter 6 it was concluded that the V-shaped asphalt floor had significantly larger values for both $A_{p}\left(1.04 \mathrm{~m}^{2}\right)$ and $D_{p}(1.5 \mathrm{~mm})$ than did the slatted and grooved floors $\left(0.76 \mathrm{~m}^{2}, 0.93 \mathrm{~mm}\right)$. For both $A_{p}$ and $D_{p}$ the variation within a farm was large, but was negligible between farms. Compared to the Tac-Rav reference values, the $A_{p}$ values were similar, but the $D_{p}$ values and variation were 3 to 6 times larger. Finally, the $D_{p}$ values in winter were significantly smaller than in spring, and with PREclean treatment the $D_{p}$ resulted in about 3 times lower values compared to the on-farm scraping if present. In short, the potential ammonia emission reduction of good floor cleaning is large.

Overall mean values were $4.27 \mathrm{~kg} \mathrm{~m}^{-3}$ for $U U N$, an initial $p H$ of 8.3 , both in fresh puddles, and a $p H(\xi)$ of 9.0 for random puddles (Chapter 7). For $U U N$ both the variation within and between farms was large, whereas the variation for $p H$ was small. The factor Diet was the only one that resulted in a significant effect, with a 0.1 difference in $p H(\xi)$, which was small. Compared to the Tac-Rav reference values, both the mean $U U N$ and $p H$ showed lower values. The calculated potential ammonia in $\mathrm{kg}_{\text {puddle }}{ }^{-1}$ showed a huge range and was considerably larger than the Tac-Rav values.

The sub-objectives 1, 2 and 3 were extensively discussed in the Chapters 2 to 7 . Sub-objective 4, however, was only limitedly dealed with in Chapter 7. Therefore, to study the effect of the values for the identified most important input variables, this general discussion starts with model calculations to estimate $\mathrm{NH}_{3}$ emissions based 
on the obtained cow house data in Chapters 3, 6 and 7. The importance of the variables in relation to the variability in modelled $\mathrm{NH}_{3}$ emission was determined and compared to the results of Chapter 2, to ultimately come up with the most important variables for the urine puddle $\mathrm{NH}_{3}$ emission. The discussion continuous with some general remarks on the cow house measurement methods and about how $\mathrm{NH}_{3}$ emission can be reduced. Finally some concluding remarks based on this discussion are drawn.

\section{2 $\mathrm{NH}_{3}$ emission model runs}

The measured values for the input variables as found in Chapters 3, 6 and 7 were used in the $\mathrm{NH}_{3}$ emission model that is currently in use by the Tac-Rav. This Tac-Rav model is the version as described by (Monteny et al., 1998), which was also part of the sensitivity analysis (Chapter 2). In the Tac-Rav model each input variable value has a fixed value and is constant during the whole model run. For example, each individual puddle that is simulated had a $p H$ of 9.4 during the whole simulation (Section 8.2.1 and Table 8.1). Based on the measured data, the values for $p H, U U N, A_{p}$ and $D_{p}$ in the performed model calculations were adapted:

1. for $p H$ a curve was used, and for $U U N, A_{p}$ and $D_{p}$ a random value was taken from their Gaussian distributions (Section 8.2.2);

2. the data per Floor-Management type (FMType) was used (Section 8.2.2);

3. the data per measurement day was used (Section 8.2.3).

To be able to use a $p H$-curve and Gaussian distributions for the other variables, the model was adapted and then verified with the original static Tac-Rav model version with reference data (Section 8.2.1).

\subsubsection{Tac-Rav model input variables with reference values}

Table 8.1 shows all Tac-Rav model input variable names and the reference values as used by the Tac-Rav. These reference values represent a dairy cow house with a concrete slatted floor and cubicles, with a slurry storage below, which is called pit. There are 100 cows, urinating 10 times a day, that result in 1000 simulated puddles per day on average by the model's puddle-generator. The cows have $3.5 \mathrm{~m}^{2} \mathrm{cow}^{-1}$ slatted-floor walking area. Each individual simulated urine puddle started with an $U U N$ of $5.0 \mathrm{~kg} \mathrm{~m}^{-3}$, which converts to $\mathrm{NH}_{3}$ (Chapter 2; Monteny et al., 1998). Puddle dimensions $\left(A_{p}\right.$ and $\left.D_{p}\right), p H$ and $T$ were fixed and they do not change in time during the simulation. The emission from the slurry pit is fixed and constant in time based on the input values. For the complete list of model assumptions see Section 1.3.

The Tac-Rav has a model-protocol that, among others, describes to perform 10 consecutive simulations of each 30 days (= 300 days) and then take the mean and SD 
Table 8.1: The Tac-Rav model input variables with name, abbreviation, reference value and units.

\begin{tabular}{|c|c|c|c|}
\hline Variable name & abbreviation & ref & units \\
\hline Number of simulations ${ }^{*}$ & - & 10 & \# consecutive simulations \\
\hline Simulation period ${ }^{*}$ & - & 30 & \#(days) simulation $^{-1}$ \\
\hline Number of cows & $N C$ & 100 & \# cows \\
\hline Urination frequency & $U F$ & 10 & $\#$ (urinations) $\operatorname{cow}^{-1} \mathrm{~d}^{-1}$ \\
\hline \multicolumn{4}{|l|}{-Floor- } \\
\hline Total floor area & $A_{f l}$ & 350 & $\mathrm{~m}^{2}$ \\
\hline Urinary urea nitrogen concentration & $U U N$ & 5.0 & $\mathrm{~kg} \mathrm{~m}^{-3}$ \\
\hline Puddle area & $A_{p}$ & 0.8 & $\mathrm{~m}^{2}$ \\
\hline Puddle depth & $D_{p}$ & 0.48 & $\mathrm{~mm}$ \\
\hline Temperature at floor level ${ }^{\dagger}$ & $T_{f l}$ & 10 & ${ }^{\circ} \mathrm{C}$ \\
\hline Air velocity just above puddle & $v_{f l}$ & 0.15 & $\mathrm{~ms}^{-1}$ \\
\hline Puddle pH & $p H_{p}$ & 9.4 & - \\
\hline \multicolumn{4}{|l|}{$-P i t^{\$}$} \\
\hline Total pit area & $A_{p i t}$ & 350 & $\mathrm{~m}^{2}$ \\
\hline TAN concentration of slurry in pit & $T A N_{\text {pit }}$ & 3.5 & $\mathrm{~kg} \mathrm{~m}^{-3}$ \\
\hline Temperature in the pit ${ }^{\ddagger}$ & $T_{\text {pit }}$ & 10 & ${ }^{\circ} \mathrm{C}$ \\
\hline Air velocity in headspace of pit & $v_{\text {pit }}$ & 0.05 & $\mathrm{~ms}^{-1}$ \\
\hline Slurry pH & $p H_{p i t}$ & 8.4 & - \\
\hline
\end{tabular}

\footnotetext{
* The Tac-Rav performs $10 \cdot 30$ days $=300$ model runs, of which they take the mean.

$\dagger$ There is no distinction between puddle and air temperature in the Tac-Rav-model.

$\ddagger$ There is no distinction between slurry and head space air temperature in the Tac-Rav-model.

$\$$ In The Netherlands there is generally a slurry storage below the whole cow house called pit. The Tac-Rav-model uses a mean $\mathrm{NH}_{3}$ emission for the whole pit surface area, based on the input values for the pit.
}

of these days. In case the dairy cows were assumed to be inside the cow house year round, the total $\mathrm{NH}_{3}$ emission of the reference cow house is $11.1 \mathrm{~kg} \mathrm{cow}^{-1} \mathrm{yr}^{-1}$. The emission from the floor is $7.7 \mathrm{~kg} \mathrm{cow}^{-1} \mathrm{yr}^{-1}(\mathrm{SD}=0.23$; Table 8.2), and from the pit is $3.4 \mathrm{~kg} \mathrm{cow}^{-1} \mathrm{yr}^{-1}(\mathrm{SD}=0.00)$.

\subsubsection{Model results per FMType}

The left part of Table 8.2 shows the input values for $p H, T_{a i r}, U U N, A_{p}$ and $D_{p}$, based on the results of the measurements (Chapters 3, 6 and 7). For the $p H$ the mean curve was used, described by Eq. (8.1) (Chapter 3).

$$
p H_{\text {puddle }}=9.16-0.38 \cdot e^{-6.63 \cdot t}-0.47 \cdot e^{-1.49 \cdot t}
$$


with the $t$ in h. The initial $p H$ was 8.31, and the final $p H$ was 9.16 . None of the experimental factors resulted in statistically significant differences for the initial $\mathrm{pH}$ of fresh urine puddles (Chapter 7). For random puddles, measured at a random moment in time, only the experimental factor Diet resulted in two statistically significant subgroups (Chapter 7). The absolute difference, however, was small. Therefore, one mean $p H$ curve for each individual puddle was used.

For $U U N$ none of the experimental factors resulted in statistically significant differences, therefore the overall or grand mean of $4.19 \mathrm{~kg} \mathrm{~m}^{-3}$ with SD was used (Chapter 7). The mean and SD of the input variables were taken from all available data at puddle level. For $T_{a i r}$ the grand mean was used as well, which is $9.7^{\circ} \mathrm{C}$ (Chapter 7). For both $A_{p}$ and $D_{p}$ the Floor-Management type (FMType) resulted in statistically significantly differences (Chapter 6 ). Therefore, the mean and SD values per FMType were used. Additionally, for $D_{p}$ the experimental factors PREclean and Season resulted in statistically significant subgroups. Only the subgroups based on PREclean were used, as this factor resulted in the largest $D_{p}$ differences, and it was directly related to an applied method in the cow house. Furthermore, the range of the PREclean-based $D_{p}$ values cover the range for Season as well.

In the adjusted Tac-Rav model an individual puddle was assigned a value for $U U N$, $A_{p}$ and $D_{p}$ that was randomly taken from their Gaussian distribution (Table 8.2). Additionally, for each puddle the $p H$-curve Eq. (8.1) started at the moment of puddle creation. For $T_{\text {air }}$ only the mean value was used. In each row in the table only the variable in that row changed to the measured value, while all others were kept at their reference value. Each model run was performed according to the Tac-Rav model-protocol of 300 days and the results are given in the right part of Table 8.2. For each row, the mean $\mathrm{NH}_{3}$ emission in $\mathrm{kg} \mathrm{cow}^{-1} \mathrm{yr}^{-1}$ was estimated with the Tac-Rav model, with SD based on the simulation days and the Coefficient of Variation (CV) was calculated. Each estimated mean emission value was divided by the Tac-Rav reference emission to determine the main effect of a single input variable. Finally, the theoretical maximum or, in other words, the potential $\mathrm{NH}_{3}$ emission $\left(P_{\mathrm{NH}_{3}}\right)$ was determined according to Eq. (8.2) (Chapters 2 and 7) and related the estimated mean divided by this potential was calculated.

$$
P_{N H_{3}}=U U N \cdot A_{p} \cdot D_{p} \cdot 10^{-3} \cdot \frac{17}{14} \cdot 10 \cdot 365
$$

with $\frac{17}{14}$ being the conversion from $\mathrm{NH}_{3}-\mathrm{N}$ to $\mathrm{NH}_{3}$ (Monteny et al., 1998). The numbers 10 and 365 represent the number of urinations per cow per day, and the number of days in a year, respectively. The emission from the pit was in all situations equal to the reference, being $3.4 \mathrm{~kg} \mathrm{cow}^{-1} \mathrm{yr}^{-1}$.

Both the $p H$ and $T_{\text {air }}$ influenced the speed of the $\mathrm{NH}_{3}$ emission process (Chapter 2). With a lower value for these variables the $\mathrm{NH}_{3}$ emission process is slower compared 
Table 8.2: The input variables $p H, T_{a i r}, U U N, A_{p}$ and $D_{p}$ with values of the measured data, being the overall mean, the mean per Floor-Management type $\left(F M T y p e^{\$}\right)$, or the mean per PREclean $\ddagger$, with SD and the Coefficient of Variation (CV). The mean and SD were taken from all available data at puddle level. This is followed by the estimated $\mathrm{NH}_{3}$ emission with the Tac-Rav model from the floor in $\mathrm{kg} \mathrm{cow}^{-1} \mathrm{yr}^{-1}$ (with $\mathrm{SD}$ at measurement day-level, and CV), followed by a percentage as compared to the reference, the potential emission (Eq. (8.2)) and the estimated mean divided by the potential.

\begin{tabular}{|c|c|c|c|c|c|c|c|c|}
\hline \multirow[b]{2}{*}{ factor } & \multirow[b]{2}{*}{$\begin{array}{c}\text { value } \\
\text { mean }(\mathrm{SD})\end{array}$} & \multirow[b]{2}{*}{$\begin{array}{l}\mathrm{CV} \\
{[\%]}\end{array}$} & \multirow[b]{2}{*}{ unit } & \multicolumn{5}{|c|}{$\mathrm{NH}_{3}$ emission } \\
\hline & & & & $\begin{array}{c}\operatorname{mean}(\mathrm{SD}) \\
{\left[\mathrm{kg} \mathrm{cow}^{-1} \mathrm{yr}^{-1}\right]}\end{array}$ & $\begin{array}{l}\mathrm{CV} \\
{[\%]}\end{array}$ & $\begin{array}{c}\text { to ref } \\
{[\%]}\end{array}$ & $\begin{array}{c}\text { potential } \\
{\left[\mathrm{kg} \mathrm{cow}^{-1} \mathrm{yr}^{-1}\right]}\end{array}$ & $\begin{array}{c}\text { mean/pot } \\
{[\%]}\end{array}$ \\
\hline Reference & Table 8.1 & - & - & $7.7(0.23)$ & 3.0 & 100.0 & 8.5 & 91 \\
\hline$p H$ & Eq. (8.1) & - & {$[-]$} & $6.8(0.18)$ & 2.7 & 88.3 & 8.5 & 80 \\
\hline$T_{a i r}$ & $9.7(4.010)$ & 41 & {$\left[{ }^{\circ} \mathrm{C}\right]$} & $7.7(0.21)$ & 2.7 & 99.5 & 8.5 & 91 \\
\hline$U U N$ & $4.19(1.758)$ & 42 & {$\left[\mathrm{~kg} \mathrm{~m}^{-3}\right]$} & $6.5(0.21)$ & 3.3 & 83.7 & 7.1 & 91 \\
\hline \multicolumn{9}{|l|}{$A_{p}^{\dagger}$} \\
\hline$S F R^{\$}$ & $0.72(0.237)$ & 33 & $\mathrm{~m}^{2}$ & $7.0(0.21)$ & 3.0 & 90.8 & 7.7 & 92 \\
\hline$G F^{\$}$ & $0.80(0.240)$ & 30 & $\mathrm{~m}^{2}$ & $7.7(0.24)$ & 3.2 & 99.9 & 8.5 & 91 \\
\hline$A F^{\$}$ & $1.04(0.310)$ & 30 & $\mathrm{~m}^{2}$ & $9.8(0.28)$ & 2.9 & 126.3 & 11.1 & 88 \\
\hline$S F C O^{\$}$ & $0.75(0.253)$ & 34 & $\mathrm{~m}^{2}$ & $7.3(0.23)$ & 3.1 & 93.8 & 8.0 & 91 \\
\hline \multicolumn{9}{|c|}{$D_{p}^{\dagger}$ (without PREclean $)^{\ddagger}$} \\
\hline$S F R^{\$}$ & $1.3(1.023)$ & 79 & $\mathrm{~mm}$ & $17.8(0.61)$ & 3.4 & 229.7 & 23.0 & 77 \\
\hline$G F^{\Phi}$ & $1.4(1.161)$ & 83 & $\mathrm{~mm}$ & $18.9(0.60)$ & 3.2 & 243.6 & 24.8 & 76 \\
\hline$A F^{\$}$ & $1.8(1.171)$ & 65 & $\mathrm{~mm}$ & $22.7(0.63)$ & 2.8 & 293.5 & 31.9 & 71 \\
\hline$S F C O^{\$}$ & $1.6(0.977)$ & 61 & $\mathrm{~mm}$ & $21.0(0.60)$ & 2.8 & 270.9 & 28.4 & 74 \\
\hline \multicolumn{9}{|c|}{$D_{p}^{\dagger}(\text { with PREclean })^{\ddagger}$} \\
\hline$S F R^{\$}$ & $0.6(0.580)$ & 97 & $\mathrm{~mm}$ & $9.6(0.37)$ & 3.8 & 124.3 & 10.6 & 91 \\
\hline$G F^{\$}$ & $0.8(0.839)$ & 105 & $\mathrm{~mm}$ & $12.3(0.43)$ & 3.5 & 159.3 & 14.2 & 87 \\
\hline$A F^{\$}$ & $0.9(0.705)$ & 78 & $\mathrm{~mm}$ & $13.3(0.49)$ & 3.7 & 171.8 & 16.0 & 83 \\
\hline$S F C O^{\$}$ & $0.8(0.806)$ & 101 & $\mathrm{~mm}$ & $12.3(0.42)$ & 3.5 & 158.6 & 14.2 & 87 \\
\hline
\end{tabular}

$\$$ FMTypes: slatted floor (reference) ( $S F R$ ), grooved floor $(G F)$, V-shaped asphalt floor $(A F)$ and slatted floor at Cows \& Opportunities farms (SFCO) (chapter 6).

$\dagger_{S F R, G F}$ and $S F C O$ were statistically significant different from AF (chapter 6).

${ }^{\ddagger} P R E$ clean is intense-floor-cleaning before puddle creation (chapter 6 ).

to higher values. The grand mean $T_{\text {air }}$ from the measurements was about equal to the reference, and as a result there was no difference in the estimated emission (both $7.7 \mathrm{~kg} \mathrm{cow}^{-1} \mathrm{yr}^{-1}$; Table 8.2). The used $p H$ curve Eq. (8.1) has lower values than the reference, especially the first hour (Chapter 3), and resulted in a lower estimated 
$\mathrm{NH}_{3}$ emission $\left(6.8 \mathrm{~kg} \mathrm{cow}^{-1} \mathrm{yr}^{-1}\right.$; Table 8.2). This was caused by the slower emission process, because puddles will be flushed away by a new puddle before its emission was finished (model assumption 3 in Section 1.3).

The $U U N, A_{p}$ and $D_{p}$ together determine the $\mathrm{NH}_{3}$ source size (Eq. (8.2); Chapters 2 and 7). Therefore it was expected that higher values directly result in higher $\mathrm{NH}_{3}$ emissions and vice versa lower values in lower emissions. This expectation was confirmed by the results (Table 8.2). The lower $U U N$ input resulted in $6.5 \mathrm{~kg} \mathrm{cow}^{-1} \mathrm{yr}^{-1}$, whereas the $\mathrm{NH}_{3}$ emission increased up to $22.7 \mathrm{~kg} \mathrm{cow}^{-1} \mathrm{yr}^{-1}$ for the largest $D_{p}$ $(1.8 \mathrm{~mm})$ for $A F$ without PREclean.

In terms of importance, the $D_{p}$ caused the largest deviation from the reference, an increase up to $294 \%$ for the $A F$ without PREclean, followed by the $U U N$ and then the $p H$. The $A_{p}$ and $T_{\text {air }}$ resulted in the smallest deviations.

The results (Table 8.2) show that a large SD for an input value does not necessarily result in a large $\mathrm{SD}$ for the estimated $\mathrm{NH}_{3}$ emission. The $\mathrm{CV}$ of the inputs varied from about $30 \%$ for $A_{p}$, to $105 \%$ for $D_{p}$, whereas the estimated emission had a fairly stable and small CV of $3 \%$ to $4 \%$.

Finally, the mean/pot-column (Table 8.2) shows the "flush-away" effect by the puddle generator (assumption 3 in Section 1.3). In the model a puddle is flushed away completely in case a new puddle is allocated to its location. This flush-effect is related to the number of urinations per day and the available floor area for them. A larger floor area $\left(A_{f l}\right)$, a smaller $A_{p}$ or both, result in more puddle locations, which result in more time for a puddle to emit. The flush-effect changes when one of the puddle related variables or the $A_{f l}$ change. The impact on the estimated $\mathrm{NH}_{3}$ emission of the flush-effect is visible by the difference between the estimated and potential emission, indicated by mean/pot (Table 8.2). These "mean/pot" values vary from $71 \%$ to $92 \%$. The flush-effect impact is the highest for the large $D_{p}$ values without PREclean, and it even ranges from $71 \%$ to $77 \%$ between the four FMTypes.

In Table 8.3 the input variable values are given as mean data per FMType with the related estimated $\mathrm{NH}_{3}$ emission from the Tac-Rav model and the emission potential. The most striking result is that the largest $\mathrm{NH}_{3}$ emission from the floor rise up to $18.5 \mathrm{~kg} \mathrm{cow}^{-1} \mathrm{yr}^{-1}$. This high emission occurred at the $A F$ farms. Besides a large $U U N$, these $A F$ farms had the largest $A_{p}$ and $D_{p}$ values, which were statistical significant (Chapter 6 ). Another striking result is the largest $U U N$ value at the farms that participate in the Cows \& Opportunities (CO) project (SFCO; $4.61 \mathrm{~kg} \mathrm{~m}^{-3}$ ) with a related high floor emission of $15.5 \mathrm{~kg} \mathrm{cow}^{-1} \mathrm{yr}^{-1}$. Based on the objective of the CO project that aimed at efficient use of nutrients at farms (Oenema et al., 2001), it was expected that these farms would have a low or even the lowest $U U N$ values. On the other hand, it is possible to have a low $U U N$ value, which is shown by the $G F$ farms $\left.\left(3.56 \mathrm{~kg} \mathrm{~m}^{-3}\right)\right)$. However, the $U U N$ was not statistically significant per FMType, due to its large variance (Chapter 7). The $S F R$, which is similar to the reference floor 
Table 8.3: The input variables $p H, T_{a i r}, U U N, A_{p}$ and $D_{p}$ with values of the measured data as mean per Floor-Management type $\left(F M T y p e^{\$}\right)$ with (SD) from puddle level data, and the related estimated mean $\mathrm{NH}_{3}$ emission with the Tac-Rav-model from the floor part in $\mathrm{kg} \mathrm{cow}^{-1} \mathrm{yr}^{-1}$ (with SD at measurement day-level), followed by the potential emission (Eq. (8.2)) and the estimated mean divided by the potential.

\begin{tabular}{|c|c|c|c|c|c|}
\hline \multirow[t]{2}{*}{ Variable } & \multirow[t]{2}{*}{ unit } & \multicolumn{4}{|c|}{ FMType } \\
\hline & & $\begin{array}{c}S F R \\
\text { mean }(\mathrm{SD})\end{array}$ & $\begin{array}{c}G F \\
\text { mean }(\mathrm{SD})\end{array}$ & $\begin{array}{c}A F \\
\text { mean }(\mathrm{SD})\end{array}$ & $\begin{array}{c}S F C O \\
\text { mean }(\mathrm{SD})\end{array}$ \\
\hline$p H$ & {$[-]$} & $9.0 \quad(0.26)$ & $8.9 \quad(0.23)$ & $9.0 \quad(0.26)$ & $9.1 \quad(0.31)$ \\
\hline$T_{a i r}$ & {$\left[{ }^{\circ} \mathrm{C}\right]$} & $9.2 \quad(4.88)$ & $8.3 \quad(3.16)$ & 11.4 & 10.6 \\
\hline$U U N$ & {$\left[\mathrm{~kg} \mathrm{~m}^{-3}\right]$} & $4.10(1.59)$ & $3.56(1.62)$ & $4.53(2.07)$ & $4.61(1.55)$ \\
\hline$D_{p}$ & {$[\mathrm{~mm}]$} & $1.3 \quad(1.02)$ & $1.4 \quad(1.16)$ & $1.8 \quad(1.17)$ & $1.6 \quad(0.98)$ \\
\hline$A_{p}$ & {$\left[\mathrm{~m}^{2}\right]$} & $0.72(0.24)$ & $0.80(0.24)$ & $1.04(0.31)$ & $0.75(0.25)$ \\
\hline $\mathrm{NH}_{3}$ emission & {$\left[\mathrm{kg} \mathrm{cow}^{-1} \mathrm{yr}^{-1}\right]$} & $10.1 \quad(0.31)$ & $8.3 \quad(0.25)$ & $18.5 \quad(0.54)$ & $15.5 \quad(0.45)$ \\
\hline$P_{N_{H}}$ & {$\left[\mathrm{~kg} \mathrm{cow}^{-1} \mathrm{yr}^{-1}\right]$} & 17.0 & 17.7 & 37.6 & 24.5 \\
\hline mean/pot & {$[\%]$} & 59 & 47 & 49 & 63 \\
\hline
\end{tabular}

$\$$ FMTypes: slatted floor (reference) $(S F R)$, grooved floor $(G F)$, V-shaped asphalt floor $(A F)$ and slatted floor at Cows \& Opportunities farms (SFCO) (chapter 6).

type of the Tac-Rav (Section 8.2.1), had a floor emission of $10.1 \mathrm{~kg} \mathrm{cow}^{-1} \mathrm{yr}^{-1}$. This was about $2.5 \mathrm{~kg} \mathrm{cow}^{-1} \mathrm{yr}^{-1}$ larger than the reference, which was fully due to the larger $D_{p}$ value $(1.3 \mathrm{~mm})$ as compared to the $D_{p}$ reference $(0.48 \mathrm{~mm})$. The $G F$ showed the lowest $\mathrm{NH}_{3}$ floor emission, which was the combined effect of the lowest $U U N$ value and a larger $A_{p}$ value as compared to the $S F R$. However, the $G F \mathrm{NH}_{3}$ emission, was still larger than the reference of the Tac-Rav. With a larger $A_{p}$ there are less puddle locations, resulting in a larger "flush-away" effect. This effect is indicated by the "mean/pot" values. They show that at the SFR and SFCO $59 \%$ to $63 \%$ of the potential $\mathrm{NH}_{3}$ emitted, while on the $G F$ and $A F$ this was lower (47\% to $49 \%$ ). For all four FMTypes the flush-away effect was larger than the reference situation of $91 \%$ (Table 8.2), mainly due to the larger $D_{p}$ values.

\subsubsection{Model results per measurement day}

In Section 8.2.2 the Tac-Rav model was used with the overall mean values of the measurement data or per FMType. In this section the mean values per measurement day were used, in other words, for the 16 Farms and 2 Seasons $=32$ measurement days (Chapters 6 and 7). Table 8.4 shows a summary of the input data per measurement day, as well as for the estimated $\mathrm{NH}_{3}$ emission. The $\mathrm{CV}$ values and thus the ranges for $U U N, D_{p}, A_{p}$ and $T_{a i r}$ were large and ranged from $21.7 \%$ for $A_{p}$ to $41.2 \%$ for $T_{\text {air }}$. The $p H$ curve (Eq. (8.1)) was not used, instead the $p H$ of 
the random puddles at a random time $(p H(\xi)$; Chapter 7) were used. these $p H(\xi)$ had a narrow range with a $\mathrm{CV}$ of only $2.2 \%$. The resulting mean $\mathrm{NH}_{3}$ emission was $14.0 \mathrm{~kg} \mathrm{cow}^{-1} \mathrm{yr}^{-1}$ with an extreme range of $3.6 \mathrm{~kg} \mathrm{cow}^{-1} \mathrm{yr}^{-1}$ to $44.3 \mathrm{~kg} \mathrm{cow}^{-1} \mathrm{yr}^{-1}$ with a CV of $68 \%$. These mean emission estimates from the floor comprehend much larger values as compared to the Tac-Rav reference $\left(7.7 \mathrm{~kg} \mathrm{cow}^{-1} \mathrm{yr}^{-1}\right.$; Table 8.2).

Table 8.4: For the input variables $U U N, D_{p}, A_{p}, p H \& T_{a i r}$, and for the estimated $\mathrm{NH}_{3}$ emission the mean, SD, CV, min and max value for the 32 measurement days (each Farm and each Season). In addition, the used values in the sensitivity analysis from Chapter 2 are shown.

\begin{tabular}{|c|c|c|c|c|c|c|c|}
\hline \multirow[b]{2}{*}{ Variable } & \multirow[b]{2}{*}{ unit } & \multirow[b]{2}{*}{ mean (SD) } & \multirow[b]{2}{*}{$\mathrm{CV}$} & \multirow[b]{2}{*}{$\min$} & \multirow[b]{2}{*}{$\max$} & \multicolumn{2}{|c|}{ Chapter 2} \\
\hline & & & & & & low & high \\
\hline$U U N$ & {$\left[\mathrm{~kg} \mathrm{~m}^{-3}\right]$} & $4.28(1.40)$ & 32.7 & 1.75 & 8.22 & 2.4 & 12.1 \\
\hline$D_{p}$ & $\mathrm{~mm}$ & $1.6 \quad(0.5)$ & 31.3 & 0.6 & 2.8 & 0.13 & 1.6 \\
\hline$A_{p}$ & $\mathrm{~m}^{2}$ & $0.83(0.18)$ & 21.7 & 0.60 & 1.26 & 0.4 & 1.8 \\
\hline$p H$ & {$[-]$} & $9.0 \quad(0.2)$ & 2.2 & 8.7 & 9.5 & 6.9 & 9.7 \\
\hline$T_{a i r}$ & {$\left[{ }^{\circ} \mathrm{C}\right]$} & $9.7 \quad(4.0)$ & 41.2 & 1.6 & 18.0 & 0.0 & 35.0 \\
\hline $\mathrm{NH}_{3}$ & {$\left[\mathrm{~kg} \mathrm{cow}^{-1} \mathrm{yr}^{-1}\right]$} & $14.0 \quad(9.5)$ & 67.9 & 3.6 & 44.3 & - & - \\
\hline
\end{tabular}

Table 8.5: The $\mathrm{R}^{2}$ for the full regression model (Eq. (8.3)), and for the full model minus one input variable.

\begin{tabular}{lc}
\hline model & $\mathrm{R}^{2}$ \\
\hline Full model & 93.3 \\
$-U U N$ & 63.3 \\
$-D_{p}$ & 82.4 \\
$-A_{p}$ & 89.7 \\
$-p H$ & 88.6 \\
$-T_{\text {air }}$ & 92.4 \\
\hline
\end{tabular}

A simple linear regression analysis was performed to analyse the variability of the estimated $\mathrm{NH}_{3}$ (Table 8.4) in relation to the five input variables, according to Eq. (8.3).

$$
N H_{3}=\text { constant }+b_{1} \cdot U U N+b_{2} \cdot D_{p}+b_{3} \cdot A_{p}+b_{4} \cdot p H+b_{5} \cdot T_{a i r}+\epsilon
$$

with a constant term and $b_{1 \ldots 5}$ as model coefficients and $\epsilon$ the residual error. This regression analysis was performed to estimate the main effect of each variable. A polynomial model was tested as well, but the result of this more complex model did not deviate from Eq. (8.3) and is therefore not presented. The correlations between 
the individual variables were already discussed in Chapters 6 and 7, therefore, they will not be included here.

Table 8.5 shows the $\mathrm{R}^{2}$ of the full regression model (Eq. (8.3)), and for the full model minus one variable at a time to indicate the importance of each variable. In each row in the table only the variable in that row was not included in the full model. Estimates for the coefficients are not given. The $\mathrm{R}^{2}$ of the full model was 93.3. Taking out the $U U N$ resulted in the largest down shift to a $\mathrm{R}^{2}$ of 63.3 , followed by the $D_{p}$ with a down shift to 89.4. The $p H$ and $A_{p}$ more or less resulted in the same $\mathrm{R}^{2}$ around 89. Finally, the $T_{\text {air }}$ resulted is a tiny down shift to a $\mathrm{R}^{2}$ of 92.4 .

\subsubsection{Model results compared to the sensitivity analysis}

The range of input variable values used in the sensitivity analysis (Chapter 2) differed from the measured range (Table 8.4):

- for $U U N$ both the measured minimum and maximum at farm level were smaller than in Chapter 2. The measured range was a little smaller as well but was still large.

- for $D_{p}$ the measured minimum and maximum at farm level was higher and the total range was larger than in Chapter 2.

- for $A_{p}, p H$ and $T_{a i r}$ the measured ranges at farm level were smaller than in Chapter 2, but for both $A_{p}$ and $T_{a i r}$ the CV was still large (20\% to $\left.41 \%\right)$. For $p H$ the $\mathrm{CV}$ was small.

The results in Sections 8.2.2 and 8.2.3 show that the $U U N$ and $D_{p}$ clearly were the two variables that caused the largest variation in $\mathrm{NH}_{3}$ emission. This large variation was directly related to the large range of input values for them that occurred to be present in commercial dairy cow houses. In the sensitivity analysis (Chapter 2) these two variables were numbers 2 and 3 in terms of importance, while the $p H$ was the number 1 . The $p H$ turned out to be quite stable in commercial dairy cow houses (Chapter 7). Therefore, it caused less variation in the estimated $\mathrm{NH}_{3}$ emission compared to the analysis in Chapter 2. The $A_{p}$ ranked 4 in both analyses. $T_{\text {air }}$ had a special meaning. The variable itself was the least important as model input, with a small contribution to the explained $\mathrm{NH}_{3}$ emission variation in both analyses compared to the other four variables. However, the important variables $U U N, D_{p}$ and, to a lesser extent, $p H$, were weakly related to $T_{a i r}$. Or to be more complete, these three variables were most likely related to the Season, of which $T_{a i r}$ was the representation. Besides $T_{a i r}$, the season contains also other effects, for example other climate conditions, farm management, floor cleanliness, feed management, and possibly even more factors. In the full model (Eq. (8.3)), all five variables had a p-value $<0.05$. 
Therefore, all five need to be measured in varying seasons in commercial dairy cow houses in order to estimate the $\mathrm{NH}_{3}$ emission with the Tac-Rav model.

The CV comparisons between the input values and the estimated $\mathrm{NH}_{3}$ emission (Table 8.2) indicate that the number of simulated puddles per day is large enough to finally end up in a mean $\mathrm{NH}_{3}$ emission value related to the mean value of the input variables. Therefore, the puddle-generator in the Tac-Rav model that generates the 1000 puddles a day (Section 8.2.1) may be simplified. On the other hand, however, the puddle-generator simulates that a puddle can be flushed away by a new puddle before its emission was finished (assumption 3 in Section 1.3). This resulted in varying "mean/pot" values (Tables 8.2 and 8.3). To summarise, it may not be possible to simplify the model up to a single "average" puddle. A simplification may be possible in case a "flush-away-factor" is introduced. Such a "flush-away-factor"may be combined with a "floor-clean-factor" to simulate a manure scraper or other floor cleaning method.

\subsection{Workability in commercial dairy cow houses}

As introduced in Sections 1.1 and 1.5, variables related to the $\mathrm{NH}_{3}$ emission process at floors in dairy cow houses are not measured in current measurement practice (Ogink et al., 2013). In the search for measurement methods for the identified five most important variables, it was directly clear that there were hardly any methods available to measure the $A_{p}$ and $D_{p}$ of fresh, on-floor urine puddles. Besides, the availability for $p H$ sensors to measure thin fluid layers was limited as well. In this thesis all methods were developed and tested both in a controlled environment of a workshop or lab and inside commercial dairy cow houses, and they all worked well (Chapters 3 to 7). In addition, it was also possible to combine methods in a single fresh puddle, except for the $D_{p}$ measurement. As discussed already in Chapter 6, to combine all methods, and to perform accurate measurements among dairy cows was quite complex. To obtain accurate data, measurements had to be performed with caution to protect the cows, the equipment, the fresh puddle and the experimentalist himself from the behaviour of curious cows. Every measurement day the sensors had to be calibrated or checked and the data had to be saved and verified. All this together made the dairy cow house measurements a quite intensive task, but demonstrates that it is possible to measure them with the requested accuracy inside commercial dairy cow houses.

Based on the measurement experiences, the most difficult task to perform among the cows was to walk around with the thermal IR camera, mounted on the trolley, in combination with the other measurements. The trolley caused a lower flexibility and speed in moving around. It also bumped on the floors that had slats, grooves or other unevenness's and that made some noise, which caused disturbances among the cows. In case a conventional, pulled manure scraper passes by, the trolley had to be carefully lifted over the scraper, or had to be lifted into a cubicle. When separating 
the $A_{p}$ measurement from the others, the $p H, U U N$ and $T_{l i q}$ measurements will be easier to perform among the cows.

In terms of measurement uncertainty, the most difficult variable to measure was the puddle depth $\left(D_{p}\right)$. The floor design and dirtiness caused a large variation of the measurement itself. Therefore, it is recommended to further develop the puddle depth measurement. However, with the currently applied method we were able to obtain $D_{p}$ values. Based on both the $D_{p}$ data and observations it is clear that puddles vary a lot in depth, which is caused by floor design and dirtiness on the floor as well. Table 8.6 show the $D_{p}(0)$ reference values currently used by the Tac-Rav in their model ranging from $0.15 \mathrm{~mm}$ to $0.58 \mathrm{~mm}$. The Tac-Rav agreed on these $D_{p}$ values, based on available literature data and expert judgement. Based on the measured results reported in this thesis (Chapter 6 ), these Tac-Rav data do not represent the real practice. Therefore, they need to be updated based on $D_{p}$ measurements in commercial dairy cow houses.

Table 8.6: Values for the puddle depth $\left(D_{p}\right)$ for various floor types and slopes as used for input in the Tac-Rav-model to estimate $\mathrm{NH}_{3}$ emission from dairy cow houses (Tac-Rav).

\begin{tabular}{lll}
\hline Floor type & Slope(s) $[\%]$ & $D_{p}(0)[\mathrm{mm}]$ \\
\hline concrete slatted floor & $0,1,2$ and 3 & $0.48,0.37,0.26$ and 0.15 \\
concrete slatted floor & globular surface & 0.20 \\
synthetic slatted floor & globular surface & 0.15 \\
solid floor & $0,1,2$ and 3 & $0.58,0.48,0.37$ and 0.27 \\
double-sloped solid floor & 1.5 and 2 & 0.37 and 0.32 \\
levelled floor, with sloped grooves & 1 & 0.37 \\
\hline
\end{tabular}

\subsection{How can $\mathrm{NH}_{3}$ be reduced in a dairy cow house?}

The focus of this $\mathrm{PhD}$ was neither on the development of $\mathrm{NH}_{3}$ emission reduction methods, nor to measure the performance of currently available reduction methods. However, the results of this thesis are of interest in relation to some reduction methods, which are addressed below.

The most important variable in $\mathrm{NH}_{3}$ emission estimation is the $U U N$ (Section 8.2.4). This $U U N$ also comprehended a large range in commercial dairy cow houses with both low and high values (Table 8.4). Therefore, it can be concluded that it is possible to have low $U U N$ values in practice. Moreover, it is possible to adjust the feed management to lower the $U U N$ values and eventually lower the $\mathrm{NH}_{3}$ emission. However, the exact feed management to lower the $U U N$ is still a challenge. The SFCO farms may have an efficient nutrient cycle (Section 8.2.2; Oenema et al., 2001), 
the $U U N$ was the largest at these farms, whereas the $G F$ farms had the lowest $U U N$. However, the urine volume per urination was not measured.

It is generally assumed that a manure scraper will lower the $\mathrm{NH}_{3}$ emission, especially in combination with a floor specially designed for this purpose. The scraper takes care of the transport of urine and faeces to the manure storage, which is generally underneath the cow house in The Netherlands. In the $\mathrm{NH}_{3}$ emission model the scraper will result in varying $A_{p}$ and $D_{p}$ values. The latest developed floor types have no or only limited openings to this so-called slurry pit to block the $\mathrm{NH}_{3}$ emission. There are completely closed floors, and the floors that have a system where slurry can enter the pit at regular distances, but where pit-air is blocked to get out. Based on these developments, clean floors were expected in practice. Clean floors, however, were not the standard in current commercial dairy cow houses as was shown in Chapter 6, and good floor cleaning still has a huge potential to lower emissions. Current available manure scrapers even mix the urine and faeces, and in this way they may even enhance the $\mathrm{NH}_{3}$ emission process. Therefore, floor designs and manure scrapers have to be further adjusted to one another and must be improved. Another option is to develop totally new floor concepts that transport urine and faeces completely separated from each other, like for example the "cow garden" with a permeable floor to drain urine and a robot that collects the faeces (Courage2025, 2016).

From the process of urea conversion to $\mathrm{NH}_{3}$ emission (Chapter 2; Ni, 1999; Monteny et al., 1998), it is known that, in case the urine and faeces are acidified to a $p H$ level $<6$, this is a very effective way to lower the $\mathrm{NH}_{3}$ emission, which was already indicated by Monteny \& Erisman (1998). With a low $p H$ the balance between ammonium and ammonia shifts to ammonium, which will stay in liquid form and will not emit. To establish a low $p H$ in a commercial dairy cow house, an acid has to be applied on the floor and in the manure storage regularly, since the cows continuously produce new fresh faeces, and slurry mixing is crucial.

Another process related option is that in case the urease is completely absent the conversion process will not take place. In other words, when fresh urine does not come into contact with active urease, there will be no $\mathrm{NH}_{3}$ emission. In current dairy cow house practice, urease is abundantly present in the faeces, which is everywhere in a cow house (Monteny \& Erisman, 1998). Research has been carried out by several persons to apply so-called urease inhibitors to stop or block the urease activity (Braam \& Swierstra, 1999; Varel et al., 2007; Parker et al., 2005; Leinker, 2007; Hagenkamp-Korth et al., 2015a,b). $\mathrm{NH}_{3}$ emission reduction effects were realised, however, similar to an acid, urease inhibitors have to be applied regularly. Besides, the urease activity really has to be lowered close to zero, otherwise it will not have an effect on the $\mathrm{NH}_{3}$ emission (Chapter 2). Therefore, to solve the $\mathrm{NH}_{3}$ emission challenge based on the urease inactivity, a solution has to be found to completely separate urine and faeces before it comes into contact with each other. 
The main disadvantage of both the use of a urease inhibitor or the use of an acid is that they end up in the slurry and eventually will enter the environment with unknown consequences. These methods can only be successfully applied in practice if the consequences are investigated and when they comply with all food security, farmer and animal safety, and environmental requirements. In addition, to control the usage of both methods, i. e. the availability, application, and storage, have to be regulated. In The Netherlands the usage of both methods is not allowed yet.

\subsection{Concluding remarks and recommendations}

Sub-objective 4 was to study the effect of the most important input variable values of fresh urine puddles on the estimated $\mathrm{NH}_{3}$ emission.

From this discussion it can be concluded that $U U N$ and $D_{p}$ resulted in the largest range of model estimated $\mathrm{NH}_{3}$ emission. Their values vary a lot with large ranges both within and between farms. Therefore, the variables $U U N$ and $D_{p}$ need to be measured in individual commercial dairy cow houses that are subject to modelling $\mathrm{NH}_{3}$ emission. These measurements need to be conducted during various seasons to incorporate season-related effects. Moreover, for each single "measurement period" more than one urine puddle has to be measured to deal with within-farm variances.

The $p H$ is fairly stable when compared to $U U N$ and $D_{p}$, both within and between farms. Nevertheless, the $p H$ remains an important variable in $\mathrm{NH}_{3}$ emission estimation. Therefore, the $p H$ has to be measured in individual cow houses in varying seasons as well to verify the current results.

The $A_{p}$ was fairly stable between farms of the same Floor-Management type (FMType), but varied within farms and it still had a significant effect on the $\mathrm{NH}_{3}$ emission. The floor design, clearly affects the $A_{p}$. Therefore, it is not necessary to measure $A_{p}$ at each individual farm, but it is sufficient to measure the $A_{p}$ only in one commercial cow house per floor design, which can be done at random.

The $T_{\text {air }}$ is of limited importance as input variable in the Tac-Rav model compared to the above mentioned four variables. However, it was still statistically significant. Besides, it is an easy variable to measure in combination with the $p H$. To summarise, $T_{\text {air }}$ can be measured in individual cow houses. In the current data the $T_{a i r}$ is a representation of the seasonal variation in the model. This variation can better be explained by a "season" factor.

Four variables can be measured at the same time in fresh urine puddles inside commercial dairy cow houses with sufficient accuracy.

1. The $p H$ can be measured by an electrode with the $p H$-sensor in the electrode-tip.

2. The $U U N$ can be determined in the lab from a collected urine sample from the floor. 
3. The $A_{p}$ can be determined from a thermal IR image of a fresh puddle by an IR algorithm.

4. The $T_{l i q}$ can be measured by a fast-responding temperature wire.

The $D_{p}$ can be measured from a manually created puddle of collected fresh urine at the floor in a commercial cow house by means of an ultrasonic sensor attached to a XY-table.

The $\mathrm{NH}_{3}$ emission model and cow houses measurements are complementary tools. Measurements can be performed more efficient and effectively guided by model calculations, and vice versa. For this purpose a good model is necessary and valuable. In this thesis, the $\mathrm{NH}_{3}$ emission model had a crucial role to identify the most important input variables. Based on this thesis, both the currently used Tac-Rav model and the measurement protocol (Ogink et al., 2013) can be updated. Of course, an updated model first needs to be validated before its part in $\mathrm{NH}_{3}$ emission estimation can be upgraded. Validation of the model needs to be done with commercial cow house data of the five identified important input variables, and $\mathrm{NH}_{3}$ emission data obtained according to the currently used measurement protocol.

During the development of the measurement methods and the protocol, the focus was to measure the five most important variables (Chapter 2) at the same time at the same puddle. Based on the results discussed here, it is probably not necessary to do this. The $A_{p}$ and $D_{p}$ were clearly related to floor design and cleanliness, whereas $U U N$ and $p H$ depend for example on farm and feed management, and both variable types were not interrelated. Therefore, the $A_{p}$ can be separated from the $U U N$ and $p H$ measurements, which will make both types of measurements easier to perform.

Between $A_{p}$ and $D_{p}$ there was no clear relation at farm level, but grouped by FMType, both variables had the largest statistically significant values at the Vshaped asphalt floor (Chapter 6 ). Like $D_{p}$, the values for $A_{p}$ varied as well, only the relative variation compared to the mean level per Farm or per FMType was smaller. Therefore, it may be fruitful to measure $A_{p}$ and $D_{p}$ simultaneously at the same puddle at different floor designs, to be able to better relate the two variables to each other and to the floor design and urine puddle variation. A puddle-creation procedure and measurement protocol have to be developed for this purpose. Determination of $A_{p}$ and $D_{p}$ values is also essential in evaluating floor scraping or cleaning performance. Floor cleaning methods must improve (Section 8.4) and therefore need to be developed, tested and finally the resulting $A_{p}$ and $D_{p}$ need to be determined.

In future measurements the $U U N$ and initial $p H$ can be obtained from urine that is directly caught from a cow, before it touches the floor, as carried out in for example the research of van Duinkerken et al. (2011). The advantage of this method is that the chance of accidentally collected dirt in the sample will be substantially lower. The disadvantage, however, is that a person has to catch urine behind a cow before it 
touches the floor, which can be difficult. In this research a cow first had to finish her urination before measurements could take place, which resulted in travel time to the urinating cow with the fresh urine. The disadvantage of the current $U U N$-method is that it can only be determined in the lab, which makes these measurements time consuming, intensive and expensive. Therefore, it would be worthwhile to develop a sensor that is able to measure the $U U N$ on-line, similar to the $p H$ sensor. With such a $U U N$ sensor it will be possible to easily measure the $U U N$ together with $p H$ of both fresh and random puddles in commercial dairy cow houses. In addition, it will be possible and it would be useful to measure $U U N$ in time series to assess the temporal development. Another option is to (automatically) capture and analyse urine on-line in the Automatic Milking System or the milking parlour.

The focus of this PhD was neither on the Milk Urea Nitrogen concentration $(M U N)$ nor on the diet. In this $\mathrm{PhD}$, only limited information was obtained for these two components. Based on the results described in Chapter 7, however, future research should focus on the diet, the $M U N$, the $U U N$, the relation between these three variables, and their relation with the $\mathrm{NH}_{3}$ emission. Van Duinkerken et al. (2003, 2005, 2011); Burgos et al. (2005, 2007, 2010) and Monteny et al. (2002) worked on these variables and relations, but the results are still limited. These results neither could fully clarify how to lower $\mathrm{NH}_{3}$ emission in commercial dairy cow houses, nor give detailed information at individual cow level. Especially values from commercial dairy cow houses are still lacking. To better understand and to lower the $U U N$ and ultimately the $\mathrm{NH}_{3}$ emission via the Diet, it is recommended to measure the $U U N$, $M U N$ and the dietary intake of several individual dairy cows in commercial farms, at least for two times a day in various seasons.

A point that has not been addressed properly is the $\mathrm{NH}_{3}$ emission related information from the slurry pit. Therefore, it is recommended to conduct additional research to get a better insight in this "black box" underneath a cow house. With new pit information, the current Tac-Rav model can certainly be improved. 



\section{References}

Aarnink, A. J. A., van den Berg, A. J., Keen, A., Hoeksma, P., \& Verstegen, M. W. A. (1996). Effect of slatted floor area on ammonia emission and on the excretory and lying behaviour of growing pigs. Journal of Agricultural Engineering Research, 64, 299-310.

Aarnink, A. J. A., \& Elzing, A. (1998). Dynamic model for ammonia volatilization in housing with partially slatted floors, for fattening pigs. Livestock Production Science, 53, 153-169.

Aarnink, A. J. A., Hol, J. M. G., Nijeboer, G. M., \& Mosquera, J. (2015). Ammoniakemissie uit varkensstallen met uitloop [Ammonia emission from pig houses with exersice yard]. Technical Report Livestock Research Rapport 868 Wageningen UR Livestock Research Wageningen.

Aarnink, A. J. A., Swierstra, D., van den Berg, A. J., \& Speelman, L. (1997). Effect of type of slatted floor and degree of fouling of solid floor on ammonia emission rates from fattening piggeries. Journal of Agricultural Engineering Research, 66, 93-102.

Aarts, H. F. M., de Haan, M. H. A., Schröder, J. J., Holster, H. C., de Boer, J. A., Reijs, J. W., Oenema, J., Hilhorst, G. J., Sebek, L. B., Verhoeven, F. P. M., Meerkerk, B., van den Pol-van Dasselaar, A., de Vliegher, A., Elgersma, A., Reheul, D., Reijneveld, J. A., Verloop, J., \& Hopkins, A. (2015). Quantifying the environmental performance of individual dairy farms - the annual nutrient cycling assessment. In Grassland and forages in high output dairy farming systems. Proceedings of the 18th Symposium of the European Grassland Federation, Wageningen, The Netherlands, 15-17 June 2015. (pp. 377-379). Wageningen Academic Publishers.

Anderson, G. A., Smith, R. J., Bundy, D. S., \& Hammond, E. G. (1987). Model to predict gaseous contaminants in swine confinement buildings. Journal of Agricultural Engineering Research, 37, 235-253. 
Arogo, J., Zhang, R. H., Riskowski, G. L., Christianson, L. L., \& Day, D. L. (1999). Mass transfer coefficient of ammonia in liquid swine manure and aqueous solutions. Journal of Agricultural Engineering Research, 73, 77-86.

Arogo Ogejo, J., Senger, R. S., \& Zhang, R. H. (2010). Global sensitivity analysis of a process-based model for ammonia emissions from manure storage and treatment structures. Atmospheric Environment, 44, 3621-3629.

Bac, C. W., Hemming, J., \& van Henten, E. J. (2014). Stem localization of sweetpepper plants using the support wire as a visual cue. Computers and Electronics in Agriculture, 105, 111-120.

Belin, É., Rousseau, D., Boureau, T., \& Caffier, V. (2013). Thermography versus chlorophyll fluorescence imaging for detection and quantification of apple scab. Computers and Electronics in Agriculture, 90, 159-163.

Ben Azouz, A., Esmonde, H., Corcoran, B., \& O'Callaghan, E. (2015). Development of a teat sensing system for robotic milking by combining thermal imaging and stereovision technique. Computers and Electronics in Agriculture, 110, 162-170.

Bland, J. M., \& Altman, D. G. (2010). Statistical methods for assessing agreement between two methods of clinical measurement. International Journal of Nursing Studies, 47, 931-936.

Blanes-Vidal, V., Sommer, S. G., \& Nadimi, E. S. (2009). Modelling surface pH and emissions of hydrogen sulphide, ammonia, acetic acid and carbon dioxide from a pig waste lagoon. Biosystems Engineering, 104, 510-521.

Blanken, K. (2007). Mestrobot verslaat rooster-schuif met gemak. Veehouderij Techniek, 10, 2.

Blunden, J., \& Aneja, V. P. (2008). Characterizing ammonia and hydrogen sulfide emissions from a swine waste treatment lagoon in North Carolina. Atmospheric Environment, 42, 3277-3290.

de Boer, I. J. M., Smits, M. C. J., Mollenhorst, H., van Duinkerken, G., \& Monteny, G. J. (2002). Prediction of Ammonia Emission from Dairy Barns Using Feed Characteristics Part I: Relation Between Feed Characteristics and Urinary Urea Concentration. Journal of Dairy Science, 85, 3382-3388.

Braam, C. R., \& van den Hoorn, C. J. (1996). Ammoniakemissie-arme betonnen stalvloeren: resultaten van experimenteel en toegepast onderzoek [Concrete floors for animal houses with low ammonia emission: results of experimental and applied research]. Technical Report IMAG-DLO Wageningen. 
Braam, C. R., Ketelaars, J. J. M. H., \& Smits, M. C. J. (1997a). Effects of floor design and floor cleaning on ammonia emission from cubicle houses for dairy cows. Netherlands Journal of Agricultural Science, 45, 49-64.

Braam, C. R., Smits, M. C. J., Gunnink, H., \& Swierstra, D. (1997b). Ammonia emission from a double-sloped solid floor in a cubicle house for dairy cows. Journal of Agricultural Engineering Research, 68, 375-386.

Braam, C. R., \& Swierstra, D. (1999). Volatilization of Ammonia from Dairy Housing Floors with Different Surface Characteristics. Journal of Agricultural Engineering Research, 72, 59-69.

van Breemen, N., Burrough, P. A., Velthorst, E. J., van Dobben, H. F., de Wit, T., Ridder, T. B., \& Reijnders, H. F. R. (1982). Soil acidification from atmospheric ammonium sulphate in forest canopy throughfall. Nature, 299, 548-550.

van Breemen, N., \& van Dijk, H. F. G. (1988). Ecosystem effects of atmospheric deposition of nitrogen in The Netherlands. Environmental Pollution, 54, 249-274.

van Bruggen, C., Groenestein, C. M., de Haan, B. J., Hoogeveen, M. W., Huijsmans, J. F. M., van der Sluis, S. M., \& Velthof, G. L. (2011). Ammoniakemissie uit dierlijke mest en kunstmest, 1990-2008; Berekeningen met het Nationaal Emissiemodel voor Ammnoniak (NEMA). Technical Report Wettelijke Onderzoekstaken Natuur \& Milieu Wageningen.

van Buggenhout, S., van Brecht, A., Eren Özcan, S., Vranken, E., Van Malcot, W., \& Berckmans, D. (2009). Influence of sampling positions on accuracy of tracer gas measurements in ventilated spaces. Biosystems Engineering, 104, 216-223.

Buijsman, E., Maas, H. F. M., \& Asman, W. A. H. (1987). Anthropogenic NH3 emissions in europe. Atmospheric Environment (1967), 21, 1009-1022.

Burgos, S. A., Embertson, N. M., Zhao, Y., Mitloehner, F. M., DePeters, E. J., \& Fadel, J. G. (2010). Prediction of ammonia emission from dairy cattle manure based on milk urea nitrogen: Relation of milk urea nitrogen to ammonia emissions. Journal of Dairy Science, 93, 2377-2386.

Burgos, S. A., Fadel, J. G., \& DePeters, E. J. (2007). Prediction of ammonia emission from dairy cattle manure based on milk urea nitrogen: Relation of milk urea nitrogen to urine urea nitrogen excretion. Journal of Dairy Science, 90, 5499-5508.

Burgos, S. A., Robinson, P. H., Fadel, J. G., \& DePeters, E. J. (2005). Ammonia volatilization potential: Prediction of urinary urea nitrogen output in lactating dairy cows. Agriculture, Ecosystems \& Environment, 111, 261-269. 
Chaoui, H., Montes, F., Rotz, C. A., \& Richard, T. L. (2009). Volatile ammonia fraction and flux from thin layers of buffered ammonium solution and dairy cattle manure. Transactions of the ASABE, 52, 1695-1706.

Clegg, S. L., \& Brimblecombe, P. (1989). Solubility of ammonia in pure aqueous and multicomponent solutions. The Journal of Physical Chemistry, 93, 7237-7248.

Click, C. N., \& Reed, J. C. (1975). Atmospheric release of hydrogen sulfide and ammonia from wet sludges and wastewaters. In Proceedings of the second national conference on complete wateReuse (pp. 426-437). Chicago, Illinois.

Cortus, E. L., Lemay, S. P., Barber, E. M., Hill, G. A., \& Godbout, S. (2008). A dynamic model of ammonia emission from urine puddles. Biosystems Engineering, 99, 390-402.

Courage2025 (2016). http://www.courage2025.nl/projecten/koeientuin.

CRC-Press (1983). Handbook of Chemistry and Physics, 64th edn. Cleveland OH: Chemical Rubber Company.

Crowe, C. T., Elger, D. F., \& Roberson, J. A. (2001). Engineering Fluid Mechanics rth Edition. John Wiley \& Sons.

Dasgupta, P. K., \& Dong, S. (1986). Solubility of ammonia in liquid water and generation of trace levels of standard gaseous ammonia. Atmospheric Environment, 20, 565-570.

DeGroot, M. A., Block, E., \& French, P. D. (2010). Effect of prepartum anionic supplementation on periparturient feed intake, health, and milk production. Journal of Dairy Science, 93, 5268-5279.

van Dooren, H. J. C., \& Galama, P. J. (2009). Internationale verkenning van ervaringen met vrijloopstallen. Technical Report Animal Sciences Group, Wageningen UR Lelystad.

van Dooren, H. J. C., \& Smits, M. C. J. (2007). Reductieopties voor ammoniak- en methaanemissie uit huisvesting voor melkvee. Technical Report November Animal Sciences Group, Wageningen UR Lelystad.

van Duinkerken, G. (2012). Personal Communication.

van Duinkerken, G., André, G., Smits, M. C. J., Monteny, G. J., Blanken, K., Wagemans, M. J. M., \& Šebek, L. B. J. (2003). Relatie tussen voeding en ammoniakemissie vanuit de melkveestal $=$ Relation between diet and ammonia emission from the dairy cow barn. Technical Report Praktijkonderzoek Veehouderij, PraktijkRapport Rundvee 25 Praktijkonderzoek Veehouderij, Wageningen UR Lelystad. 
van Duinkerken, G., André, G., Smits, M. C. J., Monteny, G. J., \& Šebek, L. B. J. (2005). Effect of rumen-degradable protein balance and forage type on bulk milk urea concentration and emission of ammonia from dairy cow houses. Journal of dairy science, 88, 1099-112.

van Duinkerken, G., Smits, M. C. J., André, G., Šebek, L. B. J., \& Dijkstra, J. (2011). Milk urea concentration as an indicator of ammonia emission from dairy cow barn under restricted grazing. Journal of dairy science, 94, 321-335.

Dumont, J., Hirvonen, T., Heikkinen, V., Mistretta, M., Granlund, L., Himanen, K., Fauch, L., Porali, I., Hiltunen, J., Keski-Saari, S., Nygren, M., Oksanen, E., Hauta-Kasari, M., \& Keinänen, M. (2015). Thermal and hyperspectral imaging for Norway spruce (Picea abies) seeds screening. Computers and Electronics in Agriculture, 116, 118-124.

ECETOC (1994). Ammonia emissions to air in western europe. Technical Report ECETOC Technical Report No. 62 European Centre for Ecotoxicology and Toxicology of Chemicals Brussels, Belgium.

Edwards, T. J., Maurer, G., Newman, J., \& Prausnitz, J. M. (1978). Vapor-Liquid Equilibria in Multicomponent Aqueous Solutions of Volatile Weak Electrolytes. AIChE Journal, 24, 966-976.

Edwards, T. J., Newman, J., \& Prausnitz, J. M. (1975). Thermodynamics of aqueous solutions containing volatile weak electrolytes. AIChE Journal, 21, 248-259.

EEA (2012). European Union emission inventory report 1990-2010 under the UNECE Convention on Long-range Transboundary Air Pollution (LRTAP). Technical Report EEA Technical Report no 8/2012 European Environment Agency.

EEA (2015). NEC Directive status report 2014. Technical Report EEA Technical report 7/2015 European Environment Agency.

Elzing, A., \& Monteny, G. J. (1997). Modeling and experimental determination of ammonia emissions rates from a scale model dairy-cow house. Transactions of the $A S A E, 40,721-726$.

Emerson, K., Russo, R. C., Lund, R. E., \& Thurston, R. V. (1975). Aqueous Ammonia Equilibrium Calculations: Effect of $\mathrm{pH}$ and Temperature. Journal of the Fisheries Research Board of Canada, 32, 2379-2383.

EU (2001). Directive 2001/81/EC of the European parliament and of the council of 23 October 2001 on national emission ceilings for certain atmospheric pollutants. 
Ferziger, J. H., \& Peric, M. (2002). Computational methods for fluid dynamics. (3rd ed.). Berlin/Heidelberg: Springer Berlin Heidelberg.

FLIR (2011). FLIR SC6xx series user's manual.

Fuller, E. N., Schettler, P. D., \& Calvin Giddings, J. (1966). A new method for prediction of binary gas-phase diffusion coefficients. Industrial \& Engineering Chemistry, 58, 18-27.

Gonzalez, R. C., \& Woods, R. E. (2008). Digital Image Processing. New Yersey, USA: Prentice Hall.

Groot Koerkamp, P. W. G., Metz, J. H. M., Uenk, G. H., Phillips, V. R., Holden, M. R., Sneath, R. W., Short, J. L., White, R. P. P., Hartung, J., Seedorf, J., Schröder, M., Linkert, K. H., Pedersen, S., Takai, H., Johnsen, J. O., \& Wathes, C. M. (1998). Concentrations and emissions of ammonia in livestock buildings in Northern Europe. Journal of Agricultural Engineering Research, 70, 79-95.

Hafner, S. D., Montes, F., \& Alan Rotz, C. (2013). The role of carbon dioxide in emission of ammonia from manure. Atmospheric Environment, 66, 63-71.

Hagenkamp-Korth, F., Haeussermann, A., \& Hartung, E. (2015a). Effect of urease inhibitor application on urease activity in three different cubicle housing systems under practical conditions. Agriculture, Ecosystems and Environment, 202, 168-177.

Hagenkamp-Korth, F., Haeussermann, A., Hartung, E., \& Reinhardt-Hanisch, A. (2015b). Reduction of ammonia emissions from dairy manure using novel urease inhibitor formulations under laboratory conditions. Biosystems Engineering, 130, 43-51.

Halachmi, I., Klopčič, M., Polak, P., Roberts, D. J., \& Bewley, J. M. (2013). Automatic assessment of dairy cattle body condition score using thermal imaging. Computers and Electronics in Agriculture, 99, 35-40.

Hales, J. M., \& Drewes, D. R. (1979). Solubility of ammonia in water at low concentrations. Atmospheric Environment, 13, 1133-1147.

Hashimoto, A. G. (1972). Ammonia desorption from concentrated chicken manure slurries, Ph.D. thesis. Ph.D. thesis Cornell University Illinois.

Haslam, R. T., Hershey, R. L., \& Kean, R. H. (1924). Effect of Gas Velocity and Temperature on Rate of Absorption. Industrial \& Engineering Chemistry, 16, 1224-1230. 
Ikeguchi, A., \& Kamo, M. (1997). Mass transfer of moisture and ammonia from manure and manure litter mixture in free-stall housing. Transactions of the ASAE, 40, 1191-1197.

Incropera, F. P., \& DeWitt, D. P. (1996). Fundamentals of heat and mass transfer. New York: John Wiley \& Sons.

IPCC (1996). Guidelines for National Greenhouse Gas Inventories.

Jayaweera, G. R., \& Mikkelsen, D. S. (1990). Ammonia volatilization from flooded soil systems: A computer model. I. Theoretical aspects. Soil Science Society of America Journal, 54, 1447-1455.

Kasper, G. J., Blanken, K., \& Bokma, S. (2010). De urease-activiteit van Comfort Slat Mats in vergelijking met betonrooster in rundveestallen [Urease activity of Comfort Slat Mats in comparison with traditional concrete slats]. Technical Report September Wageningen UR Livestock Research Lelystad.

Keesman, K. (2011). System Identification - An Introduction. (1st ed.). Springer London.

Ketelaars, J. J. M. H., \& Rap, H. (1994). Ammonia volatilization from urine applied to the floor of a dairy cow barn. In L. t Mannetje, \& J. Frame (Eds.), Grassland and society: proceedings of the 15th general meeting of the European Grassland Federation (pp. 413-417). Wageningen: Wageningen Pers.

Leinker, M. (2007). Entwicklung einer Prinziplösung zur Senkung von Ammoniakemissionen aus Nutztierställen mit Hilfe von Ureaseinhibitoren, Ph.D. thesis. Ph.D. thesis Martin-Luther-Universität Halle-Wittenberg.

Leprince, S., Barbot, S., Ayoub, F., \& Avouac, J. P. (2007). Automatic, Precise, Ortho-rectification and Coregistration for satellite Image Correlation, Application to Ground Deformation Measurement. IEEE J. Geosci. Rem. Sens., 45, 1529-1558.

Lewis, W. K., \& Whitman, W. G. (1924). Principles of gas absorption. Industrial 8 Engineering Chemistry, 16, 1215-1220.

Liang, Z. S., Westerman, P. W., \& Arogo, J. (2002). Modeling ammonia emission from swine anaerobic lagoons. Transactions of the ASAE, 45, 787-798.

Liss, P. S., \& Slater, P. G. (1974). Flux of gases across the Air-Sea interface. Nature, 247, 181-184.

Meo, R., Roglia, E., \& Bottino, A. (2012). The exploitation of data from remote and human sensors for environment monitoring in the SMAT project. Sensors, 12, 17504-17535. 
Monteny, G. J. (2000). Modelling of ammonia emissions from dairy cow houses, Ph.D thesis. Ph.D. thesis Wageningen University Wageningen.

Monteny, G. J., \& Erisman, J. W. (1998). Ammonia emission from dairy cow buildings: A review of measurement techniques, influencing factors and possibilities for reduction. Netherlands Journal of Agricultural Science, 46, 225-247.

Monteny, G. J., Schulte, D. D., Elzing, A., \& Lamaker, E. J. J. (1998). A conceptual mechanistic model for the ammonia emissions from free stall cubicle dairy cow houses. Transactions of the ASAE, 41, 193-201.

Monteny, G. J., Smits, M. C. J., van Duinkerken, G., Mollenhorst, H., \& de Boer, I. J. M. (2002). Prediction of ammonia emission from dairy barns using feed characteristics part II: Relation between urinary urea concentration and ammonia emission. Journal of Dairy Science, 85, 3389-3394.

Montes, F., Rotz, C. A., \& Chaoui, H. (2009). Process modeling of ammonia volatilization from ammonium solution and manure surfaces: a review with recommended models. Transactions of the ASABE, 52, 1707-1720.

Mosquera, J., Hol, J. M. G., \& Hofschreuder, P. (2005a). Gasvormige emissies uit het melkveebedrijf van de familie Spruit III. Mestopslag buiten de stal. Technical Report Agrotechnology \& Food Innovations B.V. Wageningen UR Wageningen.

Mosquera, J., Hol, J. M. G., \& Huis in 't Veld, J. W. H. (2008). Meetmethode voor ammoniakemissiemetingen uit stallen met uitloop. Technical Report Animal Sciences Group, Wageningen UR Lelystad.

Mosquera, J., Kasper, G. J., Blanken, K., Dousma, F., \& Aarnink, A. J. A. (2010). Ontwikkeling snelle meetmethode ter bepaling van ammoniakemissiereductie van vloergebonden maatregelen $=$ Development of a fast measurement method for the determination of ammonia emission reduction from floor related measures. Technical Report September Wageningen UR Livestock Research Lelystad.

Mosquera, J., Monteny, G. J., \& Erisman, J. W. (2005b). Overview and assessment of techniques to measure ammonia emissions from animal houses: the case of the Netherlands. Environmental Pollution, 135, 381-388.

Muck, R. E. (1982). Urease Activity in Bovine Feces. Journal of Dairy Science, 65, 2157-2163.

Muck, R. E., \& Steenhuis, T. S. (1981). Nitrogen losses in free stall dairy barns. In Livestock Wastes: A Renewable Resource (pp. 406-409). St. Joseph, Michigan: ASAE. 
Ni, J. Q. (1999). Mechanistic models of ammonia release from liquid manure: A review. Journal of Agricultural and Engineering Research, 72, 1-17.

Ni, J. Q., Hendriks, J., Vinckier, C., \& Coenegrachts, J. (2000). Development and Validation of a Dynamic Mathematical Model of Ammonia Release in Pig House. Environment International, 26, 105-115.

Norton, T., Sun, D. W., Grant, J., Fallon, R., \& Dodd, V. (2007). Applications of computational fluid dynamics (CFD) in the modelling and design of ventilation systems in the agricultural industry: A review. Bioresource Technology, 98, 23862414 .

Oenema, J., Koskamp, G. J., \& Galama, P. J. (2001). Guiding commercial pilot farms to bridge the gap between experimental and commercial dairy farms; the project Cows \& Opportunities. NJAS - Wageningen Journal of Life Sciences, 49, 277-296.

Oenema, O., Oudendag, D. A., Witzke, H. P., Monteny, G. J., Velthof, G. L., Pietrzak, S., Pinto, M., Britz, W., Schwaiger, E., Erisman, J. W., Vries, W. D., van Grinsven, J. J. M., \& Sutton, M. (2007). Integrated measures in agriculture to reduce ammonia emissions; final summary report. Technical Report Alterra, Wageningen UR Wageningen.

Ogink, N. W. M., \& Kroodsma, W. (1996). Reduction of ammonia emission from a cow cubicle house by flushing with water or a formalin solution. Journal of Agricultural Engineering Research, 63, 197-204.

Ogink, N. W. M., Mosquera, J., \& Hol, J. M. G. (2011). Protocol voor meting van ammoniakemissie uit huisvestingssystemen in de veehouderij $2010=$ Measurement protocol for ammonia emission from housing systems in livestock production 2010. Technical Report Livestock Research Rapport 454 Wageningen UR Livestock Research Lelystad.

Ogink, N. W. M., Mosquera, J., \& Hol, J. M. G. (2013). Protocol voor meting van ammoniakemissie uit huisvestingssystemen in de veehouderij $2013=$ Measurement protocol for ammonia emission from housing systems in livestock production 2013. Technical Report Livestock Research Rapport 726 Wageningen UR Livestock Research.

Olesen, J. E., \& Sommer, S. G. (1993). Modeling Effects of Wind-Speed and Surface Cover on Ammonia Volatilization from Stored Pig Slurry. Atmospheric Environment, 27, 2567-2574.

van Ouwerkerk, E. N. J. (1993). Meetmethoden NH3-emissie uit stallen. Wageningen: IMAG-DLO. 
Parker, D. B., Pandrangi, S., Greene, L. W., Almas, L. K., Cole, N. A., Rhoades, M. B., \& Koziel, J. A. (2005). Rate and frequency of urease inhibitor application for minimizing ammonia emissions from beef cattle feedyards. Transactions of the American Society of Agricultural Engineers, 48, 787-793.

Payne, R., Welham, S., \& Harding, S. (2015). A guide to REML in Genstat (18th edition). (Genstat 18 ed.). Hemel Hempstead: VSN International.

PBL (2012). Balans van de leefomgeving 2012. Den Haag: Planbureau voor de Leefomgeving.

Phillips, V. R., Lee, D. S., Scholtens, R., Garland, J. A., \& Sneath, R. W. (2001). A Review of Methods for measuring Emission Rates of Ammonia from Livestock Buildings and Slurry or Manure Stores, Part 2: monitoring Flux Rates, Concentrations and Airflow Rates. Journal of Agricultural Engineering Research, 78, 1-14.

Phillips, V. R., Scholtens, R., Lee, D. S., Garland, J. A., \& Sneath, R. W. (2000). A Review of Methods for Measuring Emission Rates of Ammonia from Livestock Buildings and Slurry or Manure Stores, Part 1: Assessment of Basic Approaches. Journal of Agricultural Engineering Research, 77, 355-364.

Poteko, J., Schrade, S., Steiner, B., \& Zähner, M. (2014). Development and validation of a measuring method for quantifying the residual soiling mass after the removal of dung from solid floor surfaces, and results of comparative measurements at pilotplant scale. In International Conference on Agricultural Engineering (p. 8). Zurich, Switzerland.

Sander, R. (1999). Modeling Atmospheric Chemistry: Interactions between Gas-Phase Species and Liquid Cloud/Aerosol Particles. Surveys in Geophysics, 20, 1-31.

Scholtens, R., \& Huis in 't Veld, J. W. H. (1997). Praktijkonderzoek naar de ammoniakemissie van stallen XXXVI. Natuurlijk geventileerde ligboxenstal met betonroosters voor melkvee. Technical Report Rapport 97-1006 IMAG-DLO Wageningen.

Scholtens, R., van der Heiden - de Vos, J. J. C., \& Huis in 't Veld, J. W. H. (1996). Natuurlijk geventileerde ligboxenstal voor melkvee met hellende dichte vloer en zelfrijdende sproeischuiven. Technical Report IMAG-DLO Wageningen.

Schrade, S., Zeyer, K., Gygax, L., Emmenegger, L., Hartung, E., \& Keck, M. (2012). Ammonia emissions and emission factors of naturally ventilated dairy housing with solid floors and an outdoor exercise area in Switzerland. Atmospheric Environment, 47, 183-194. 
Smits, M. C. J., van Dousma, F., Kupers, G. C. C., \& Blanken, K. (2009). Orienterend laboratoriumonderzoek naar ammoniakemissie uit bodempakketten voor vrijloopstallen. Technical Report Animal Sciences Group, Wageningen UR Lelystad.

Snoek, D. J. W., Haesen, G. P. M. J., Groot Koerkamp, P. W. G., \& Monteny, G. J. (2010). Effect of floor design in a dairy cow house on ammonia emission - design, test and preliminary results with an experimental set-up for run off experiments. In International Conference on Agricultural Engineering (pp. 1-10). Clermont-Ferrand, France: Cemagref.

Snoek, D. J. W., Hofstee, J. W., van Dueren den Hollander, A. W., Vernooij, R. E., Ogink, N. W. M., \& Groot Koerkamp, P. W. G. (2016a). Quantification of simulated cow urine puddle areas using a thermal IR camera. revised version submitted for publication, -, - .

Snoek, D. J. W., Ogink, N. W. M., Stigter, H., \& Groot Koerkamp, P. W. G. (2012). Sensitivity Analysis of a Mechanistic Model for the Ammonia Emission of Dairy Cow Houses. 2012 IX International Livestock Environment Symposium (ILES IX), .

Snoek, D. J. W., Ogink, N. W. M., Stigter, J. D., Agricola, S., van de Weijer, T. M., \& Groot Koerkamp, P. W. G. (2016b). Dynamic behavior of $\mathrm{pH}$ in fresh urine puddles of dairy cows. Transactions of the ASABE, 59,--

Snoek, D. J. W., Stigter, J. D., Blaauw, S. K., Groot Koerkamp, P. W. G., \& Ogink, N. W. M. (2016c). Assessing fresh urine puddles in commercial dairy cow houses part 1: surface area and depth. submitted for publication, -, - .

Snoek, D. J. W., Stigter, J. D., Kupers, G. C. C., Groot Koerkamp, P. W. G., \& Ogink, N. W. M. (2016d). Assessing fresh urine puddles in commercial dairy cow houses part 2: $\mathrm{pH}$, urea concentration, and ammonia emission potential. submitted for publication, -, - .

Snoek, D. J. W., Stigter, J. D., Ogink, N. W. M., \& Groot Koerkamp, P. W. G. (2014a). IR-camera method to determine urine puddle area in dairy cow houses. In International Conference on Agricultural Engineering (p. 8). Zurich, Switzerland.

Snoek, D. J. W., Stigter, J. D., Ogink, N. W. M., \& Groot Koerkamp, P. W. G. (2014b). Sensitivity analysis of mechanistic models for estimating ammonia emission from dairy cow urine puddles. Biosystems Engineering, 121, 12-24.

Snoek, D. J. W., Stigter, J. D., Ogink, N. W. M., \& Groot Koerkamp, P. W. G. (2015). Measurement method for urine puddle depth in dairy cow houses as input variable for ammonia emission modelling. Agricultural Engineering International: CIGR Journal, Special Issue 18th World Congress of CIGR, 30-36. 
Sommer, S. G., \& Sherlock, R. R. (1996). pH and buffer component dynamics in the surface layers of animal slurries. The Journal of Agricultural Science, 127, 109-116.

Spek, J. W., Bannink, A., Gort, G., Hendriks, W. H., \& Dijkstra, J. (2012a). Effect of sodium chloride intake on urine volume, urinary urea excretion, and milk urea concentration in lactating dairy cattle. Journal of Dairy Science, 95, 7288-7298.

Spek, J. W., Bannink, A., Gort, G., Hendriks, W. H., \& Dijkstra, J. (2013a). Interaction between dietary content of protein and sodium chloride on milk urea concentration, urinary urea excretion, renal recycling of urea, and urea transfer to the gastrointestinal tract in dairy cows. Journal of dairy science, 96, 5734-45.

Spek, J. W., Dijkstra, J., van Duinkerken, G., \& Bannink, A. (2012b). A review of factors influencing milk urea concentration and its relationship with urinary urea excretion in lactating dairy cattle. The Journal of Agricultural Science, (pp. 407-423).

Spek, J. W., Dijkstra, J., van Duinkerken, G., Hendriks, W. H., \& Bannink, A. (2013b). Prediction of urinary nitrogen and urinary urea nitrogen excretion by lactating dairy cattle in northwestern Europe and North America: a meta-analysis. Journal of dairy science, 96, 4310-22.

Srinath, E. G., \& Loehr, R. C. (1974). Ammonia desorption by diffused aeration. Journal of the Water Pollution Control Federation, 46, 1939-1957.

Swierstra, D., Braam, C. R., \& Smits, M. C. (2001). Grooved floor system for cattle housing: Ammonia emission reduction and good slip resistance. Applied Engineering in Agriculture, 17, 85-90.

Swierstra, D., Smits, M. C. J., \& Braam, C. R. (1997). Grooved concrete floor elements to reduce ammonia emission and to prevent slipperiness in loose houses for cows. In Proceedings of Concrete for a Sustainable Agriculture (p. 7). Stavanger, Norway.

Swierstra, D., Smits, M. C. J., \& Kroodsma, W. (1995). Ammonia emission from cubicle houses for cattle with slatted and solid floors. Journal of Agricultural Engineering Research, 62, 127-132.

Tac-Rav (2016). http://www.infomil.nl/onderwerpen/landbouwtuinbouw/ammoniak/rav/stalbeschrijvingen/.

UNECE (1999). The 1999 Gothenburg Protocol to Abate Acidification. Eutrophication and Ground-level Ozone.

Vaddella, V. K., Ndegwa, P. M., \& Jiang, A. (2011). An empirical model of ammonium ion dissociation in liquid dairy manure. Transactions of the ASABE, 54, 1119-1126. 
Vaddella, V. K., Ndegwa, P. M., Ullman, J. L., \& Jiang, A. (2013). Mass transfer coefficients of ammonia for liquid dairy manure. Atmospheric Environment, 66, 107-113.

Varel, V. H., Wells, J. E., \& Miller, D. N. (2007). Combination of a urease inhibitor and a plant essential oil to control coliform bacteria, odour production and ammonia loss from cattle waste. Journal of Applied Microbiology, 102, 472-477.

Velthof, G. L., van Bruggen, C., Groenestein, C. M., de Haan, B. J., Hoogeveen, M. W., \& Huijsmans, J. F. M. (2012). A model for inventory of ammonia emissions from agriculture in the Netherlands. Atmospheric Environment, 46, 248-255.

Wang, C., Li, B., Zhang, G., Rom, H. B., \& Strøm, J. S. (2006). Model estimation and measurement of ammonia emission from naturally ventilated dairy cattle buildings with slatted floor designs. Journal of the Air and Waste Management Association, $56,1252-1259$.

Wu, W., Kai, P., \& Zhang, G. (2012). An assessment of a partial pit ventilation system to reduce emission under slatted floor - Part 1: Scale model study. Computers and Electronics in Agriculture, 83, 127-133.

Ye, Z., Zhang, G., Seo, I. H., Kai, P., Saha, C. K., Wang, C., \& Li, B. (2009). Airflow characteristics at the surface of manure in a storage pit affected by ventilation rate, floor slat opening, and headspace height. Biosystems Engineering, 104, 97-105.

Zevenbergen, G. (2009). Mestrobot sproeit water - Jelle Bouma scoort maatlatpunten. Veehouderij Techniek, 12, 32-34.

Zhang, R. H., Day, D. L., Christianson, L. L., \& Jepson, W. P. (1994). A Computer Model for Predicting Ammonia Release Rates from Swine Manure Pits. Journal of Agricultural Engineering Research, 58, 223-229.

Zhixiong, L., Nan, C., Perdok, U. D., \& Hoogmoed, W. B. (2005). Characterisation of Soil Profile Roughness. Biosystems Engineering, 91, 369-377. 



\section{Summary}

In the late nineties, the EU National Emission Ceiling Directive (NECD), among others, set $\mathrm{NH}_{3}$ emission ceilings EU wide and for each member country, for the year 2010. From 1990 to 2010 the ammonia $\left(\mathrm{NH}_{3}\right)$ emission reduced EU wide with $28 \%$ and the set targets were achieved by each member country (EEA, 2012). However, the European Commission (EC) proposed a new European Clean Air Programme with an updated NECD for the year 2020 and beyond (EEA, 2015). In addition, they reported that agriculture still dominates the $\mathrm{NH}_{3}$ emission, and that compared to other pollutants the $\mathrm{NH}_{3}$ emissions did not decrease to the same extent since 1990. In The Netherlands, the $\mathrm{NH}_{3}$ emission from dairy cow houses is one of the most important contributors (Velthof et al., 2012).

Over the past 25 years a lot of research has been conducted to understand and model the $\mathrm{NH}_{3}$ emission from dairy cow houses (Elzing \& Monteny, 1997), and to measure it (Mosquera et al., 2005b). In addition, reduction measures were identified (e.g. Monteny \& Erisman, 1998) and further developed for implementation in commercial dairy cow houses, which is regulated by the Ministry of Infrastructure and Environment, supported by a Technical Advisory Committee (Tac-Rav). The Tac-Rav, among other sources of information, makes use of the $\mathrm{NH}_{3}$ emission model developed by Monteny et al. (1998) to pre-assess new cow housing systems. However, still some important aspects remain unclear. For example, the values for the input variables of this Tac-Rav model and the relations with the $\mathrm{NH}_{3}$ emission are limitedly known. Moreover, these input variables are not measured in practice, since they are not included in the currently applied measurement protocol (Ogink et al., 2013). Therefore, the introduction and (pre)-assessment of new reduction methods is complicated and subject to a high level of uncertainty. Besides, the functioning of applied reduction measures in commercial dairy cow houses is limitedly known.

In this thesis the most important input variables and process parameters were identified in current available mechanistic $\mathrm{NH}_{3}$ emission models and theory. To measure these variables in practice, measurement methods were explored and developed. Then the values and interactions of the identified variables were assessed for fresh urine 
puddles in commercial dairy cow houses. Finally, the effect of these input variable values on the estimated $\mathrm{NH}_{3}$ emission was studied.

In Chapter 2 it was concluded that five urine puddle related variables explained at least $71 \%$ of the variation in the model estimated $\mathrm{NH}_{3}$ emisison from the floor in dairy cow houses. These variables are, in order of importance, puddle $p H$, depth $\left(D_{p}\right)$, urinary urea nitrogen concentration $(U U N)$, surface area $\left(A_{p}\right)$, and temperature $\left(T_{l i q}\right)$. The model parameters did not show an effect: i. e.the dissociation constant, the Henry's law constant and the mass transfer coefficient. The remaining four variables were the air temperature $\left(T_{a i r}\right)$ and air velocity $(v)$ just above a puddle, the maximum rate of urea conversion or urease activity $(S m)$, and the Michaelis-Menten constant $(K m)$. For each input variable in the model the available data was scarce, and it was therefore recommended to measure the five most important variables in practice. The main cause for the data scarcity was the lack of accurate sensors and the complex circumstances to measure the selected five variables in commercial dairy cow houses. Therefore, possible useful sensors were explored and measurement methods were developed in Chapters 3, 4 and 5.

In Chapter 3 both a $p H$ sensor and a temperature sensor were selected and tested that were able to measure the $\mathrm{pH}$ and temperature of thin fluid layers, like urine puddles on floors. In addition, in total 26 fresh dairy cow urine puddles in commercial dairy cow houses were measured in $4 \mathrm{~h}$ time series to investigate $\mathrm{pH}$ values and dynamic behaviour. From the $p H$ series a mean $p H$ curve was distinguished that represents a urine puddle. The curve starts at an initial $p H$ value of 8.3 and increases to 9.2 after $4 \mathrm{~h}$. Ninety percent of this increase is realised within the first hour.

Chapter 4 describes the measurement method that was developed and tested in the lab to obtain the $D_{p}$ values from urine puddles in commercial dairy cow houses. A so-called XY-table with an ultrasonic distance sensor attached to it was used, and compared to the balance method being defined as the "gold standard". The depth of water puddles was successfully measured in various experimental setups and the ultrasonic method achieved a measurement uncertainty smaller than $0.1 \mathrm{~mm}$.

To measure the $A_{p}$, a thermal IR camera was used. In a preliminary experiment in two cow houses, the IR camera was mounted on a movable trolley and was able to collect IR images of fresh urine puddles. In Chapter $\mathbf{5}$ an IR model was developed to obtain the $A_{p}$ value automatically from an IR image based on the temperature values of an individual puddle and its near surroundings. A lab experiment was performed with manually created warm and blue-coloured water puddles on three floor types. Simultaneously both an IR and RGB image were taken from a puddle and the $A_{p}$ was determined by the IR model $\left(A_{p, I R}\right)$ and was compared to the ground truth reference $\left(A_{p, G T}\right)$ based on the assessment of the RGB image by three individuals. The $A_{p, I R}$ underestimated the $A_{p, G T}$ by $2.53 \%$ for which is compensated in the model $A_{p, G T}=1.0253 \cdot A_{p, I R}$. This regression model has a zero y-intercept and the standard 
deviation of the residuals was only $0.0651 \mathrm{~m}^{2}$ yielding an uncertainty smaller than $0.1 \mathrm{~m}^{2}$. In addition, the $A_{p, I R}$ was not affected by the mean background temperature.

The selected sensors and developed methods were combined and a protocol was described to measure the variables $p H, D_{p}, U U N, A_{p}$ and $T_{l i q}$ of fresh, random and manually created urine puddles in commercial dairy cow houses. In a factorial experimental setup based on four floor-management types (FMTypes), with experimental factors Season and PREclean treatment. PREclean was only applied to the $D_{p}$ measurement and represented intense-floor-cleaning before urine puddle creation, which was compared with those created under normal floor conditions with on-farm manure scraping. Two explanatory factors were distinguished that were not controlled at farm level and were not equally spread among the FMTypes, being Diet and Calc.

In Chapter 6 it was concluded that the V-shaped asphalt floor had significantly larger values for both $A_{p}\left(1.04 \mathrm{~m}^{2}\right)$ and $D_{p}(1.5 \mathrm{~mm})$ than did the slatted and grooved floors $\left(0.76 \mathrm{~m}^{2}, 0.93 \mathrm{~mm}\right)$. For both $A_{p}$ and $D_{p}$ the variation within a farm was large, but was negligible between farms. Compared to the Tac-Rav reference values, the $A_{p}$ values were similar, but the $D_{p}$ values and variation were 3 to 6 times larger. Finally, the $D_{p}$ values in winter were significantly smaller than in spring, and with PREclean treatment the $D_{p}$ resulted in about 3 times lower values compared to the on-farm scraping if present. In short, the potential ammonia emission reduction of good floor cleaning is large.

Overall mean values were $4.27 \mathrm{~kg} \mathrm{~m}^{-3}$ for $U U N$, an initial $p H$ of 8.3 , both in fresh puddles, and a $p H(t=\xi)$ of 9.0 for random puddles at a random time (Chapter 7). For $U U N$ both the variation within and between farms was large, whereas the variation for $p H$ was small. The factor Diet was the only one that resulted in a significant effect, with a 0.1 difference in $p H(\xi)$, which was small. Compared to the Tac-Rav reference values, both the mean $U U N$ and $p H$ showed lower values. The calculated potential ammonia in $\mathrm{kg}_{\text {puddle }}{ }^{-1}$ showed a huge range and was considerably larger than the Tac-Rav values for the reference situation.

The general discussion of this thesis, presented in Chapter 8 , discusses three different topics First the measured variable values from the dairy cow house measurement were substituted in the currently used model (Tac-Rav) and the $\mathrm{NH}_{3}$ emission was determined. These results were compared to the outcome of the sensitivity analysis in chapter 2 to ultimately come up with the most important variables for the urine puddle $\mathrm{NH}_{3}$ emission. It is shown that the $U U N$ range at farm level is both slightly smaller and shifts to slightly lower values than the $U U N$ data used in chapter 2, while for $D_{p}$ the range and values are both larger. These two variables causes the largest variation in the estimated $\mathrm{NH}_{3}$ emissions, and not the $p H$. In conclusion, these two variables need to be measured in individual commercial dairy cow houses to determine the $\mathrm{NH}_{3}$ emission. For $A_{p}, p H$ and $T_{\text {air }}$ the measured ranges at farm level are less large than those in chapter 2. The $p H$ turns out to be fairly stable in commercial cow 
houses and, related to that, it causes less variation in the estimated $\mathrm{NH}_{3}$ emission. Nevertheless, the $p H$ still ranks as the third most important variable, and therefore needs to be measured in individual cow houses. The $A_{p}$ is fairly stable between farms, but varies within farms and it still has a significant effect on the $\mathrm{NH}_{3}$ emission. The floor design, clearly affects the $A_{p}$. Therefore, it is not necessary to measure $A_{p}$ at each individual farm, but it is sufficient to measure the $A_{p}$ only in one commercial cow house per floor design. The $T_{\text {air }}$ variable is of limited importance as input variable in the Tac-Rav model compared to the above mentioned four variables, but is still significant. In the data the $T_{a i r}$ represents the seasonal variation in the model.

As a second topic, the usability of the measurement methods is discussed. All measurement methods worked in practice with the required accuracy. The $A_{p}$ measurement was the most difficult one to perform. Based on the results, the $A_{p}$ measurement can be separated from the others, and in this way the $U U N, p H$ and $T_{a i r}$ measurements will be easier to perform in practice. The $D_{p}$ measurement shows the largest uncertainty. The floor design and dirtiness caused a large variation of the measurement itself. Therefore, it is recommended to further develop the puddle depth measurement equipment. In addition, it will be fruitful to measure $A_{p}$ and $D_{p}$ simultaneously at the same urine puddle at different floor designs, to better relate the two variables to each other and to the floor design and urine puddle variation. Finally, it is worthwhile to develop a sensor that is able to measure the $U U N$ on-line, similar to the $p H$ sensor. With such a $U U N$ sensor it will be possible to easily measure the $U U N$ together with $p H$ of both fresh and random puddles in commercial dairy cow houses.

As a third topic, the options to reduce $\mathrm{NH}_{3}$ emission in commercial dairy cow houses are discussed. It was not the focus of this thesis to fully describe all possible and available reduction methods, but some were addressed. A low $U U N$ value results in a lower $\mathrm{NH}_{3}$ emission, and low $U U N$ values are possible in practice. Based on $\mathrm{NH}_{3}$ emission process theory, the emission can be reduced by either blocking the urease activity with a urease inhibitor, or to lower the $p H$ to a level $<6$ by adding an acid to the urine and faeces. It has been demonstrated already that both methods work. However, urease inhibitor or acid need to be applied regularly since cows continuously produce urine and faeces and these acids will eventually enter the environment. Therefore, the usage of both methods needs to be regulated. In The Netherlands both methods are not allowed yet. A solution without adding chemicals, is to separate urine and faeces for $100 \%$. Current floor designs and manure scrapers are not capable to accomplish this level of separation. Therefore, to reach this goal, manure handling systems must be improved. 


\section{Dankwoord}

Mijn PhD afgerond! Het voelt ook echt als "mijn" PhD. Maar zonder steun en begeleiding had ik deze thesis wellicht niet kunnen afronden. Dus graag wil ik enkele belangrijke personen bedanken.

Allereerst de man die er hoogstpersoonlijk voor gezorgd heeft dat ik überhaupt ooit begonnen ben aan dit PhD traject, namelijk Peter Groot Koerkamp. Als begeleider tijdens mijn MSc thesis over urine afvoerend vermogen van stalvloeren, vroeg hij me eens iets in de trent van: "Zou een $\mathrm{PhD}$ niet iets voor jou zijn? Onderzoek doen naar ammoniak in melkveestallen." en zo geschiedde. Peter, ik ken je inmiddels al behoorlijk lang, onze kennismaking begon ergens in 2007 voor mijn BSc thesis, en er was wel een match. Hoewel er periodes waren dat we elkaar minder spraken, kon ik altijd bij je terecht met vragen, opmerkingen of gewoon voor een praatje. Je gaf me meteen grote verantwoordelijkheden, op gebied van onderwijs bijvoorbeeld, maar je had vertrouwen in me vanaf de start en dat was fijn. Peter, bedankt.

In september 2010 maakte ik kennis met Nico Ogink. Nico had samen met Peter het idee van mijn $\mathrm{PhD}$ project uitgezet als een samenwerking tussen de Farm Technology Group (FTE) en Wageningen UR Livestock Research (WLR) en Nico werd mijn dagelijkse begeleider. Nico, ik heb je altijd als een slimme en ervaren persoon gezien waar ik veel van kon leren, met toch wel gewoon een nuchtere aanpak. Dit paste mij wel, en dit werkte prima. Vele woensdagen heb ik bij je in kantoor gezeten, wat ik erg prettig vond want zo sprak ik je regelmatig en we konden makkelijk even ergens over discussiëren. Nico, bedankt.

Toen ik begon was er nog weinig tot niets uitgewerkt voor mijn PhD. Samen met Peter en Nico heb ik mijn PhD opgezet en gepland. In het originele PhD-voorstel was het plan dat ik het ammoniak emissiemodel zou herzien en bij de tijd zou brengen. Voor extra support op dit gebied werd Hans Stigter van Biometris bij mijn PhD betrokken. Uiteindelijk ben ik zelf nooit toegekomen aan een "model-V2.0", maar ondanks dat was Hans een welkome toevoeging. Hans, als melkvee-leek stelde je altijd vragen bij van alles en nog wat, en vooral "waarom?" en "kunnen we het modelleren?" Dit zorgde ervoor dat we niet zomaar iets deden, maar dat we over alle experimenten 
goed nagedacht hebben met in het achterhoofd het ammoniak emissiemodel. Is er dan helemaal niets gemodelleerd? Zeker wel, namelijk in hoofdstuk 2 is het huidige model aan de tand gevoeld en in hoofdstuk 3 hebben we de $\mathrm{pH}$ gemodelleerd. Hans, bedankt.

Net zo belangrijk voor eigenlijk al mijn werk wat ik bij de leerstoel FTE gedaan heb was de allround ondersteuning van Sam Blaauw. Sam, volgens mij heb jij bij ongeveer alles wat ik gedaan heb wel een rol gespeeld. Alle FTE-pc's parallel inschakelen voor mijn gevoeligheidsanalyse? Sam regelt het. Meetapparatuur, die nog niet bestaat, bedenken en organiseren? Sam denkt mee. Tussen de koeien testen en meten? Sam gaat mee. Daarnaast hebben we samen, wat mij betreft zeer succesvol, $6 \mathrm{x}$ het vak Engineering Design gedoceerd. Sam, jij had altijd goede ideeën en dit heeft zeer zeker bijgedragen aan mijn $\mathrm{PhD}$. Sam, bedankt.

Nog zo'n belangrijke steunpilaar was Bastiaan Vroegindeweij. Bastiaan, sinds onze BSc thesis in 2007 zitten wel al ongeveer non-stop bij elkaar in kantoor, en eigenlijk al sinds onze studie-start in 2004. Hoewel we op behoorlijk wat vlakken als water en vuur zijn, zorgt dit juist voor goede aanvullingen aan elkaar denk ik. Verder heb ik er eigenlijk geen woorden voor. Bastiaan, bedankt.

Also thanks to all my colleague PhD's. We had a nice time in the office, but also outside. For example, we had pleasant PhD-dinners once a month for quite some time. Liansun, thanks for the very nice trip to China we made together! Peter, thanks for the latex introduction and support, and being the handyman. Suh, thanks for your hospitality and your drive in being a $\mathrm{PhD}$.

Verder wil ik al mijn collega's bij zowel FTE als WLR bedanken. Ik heb me op beide plekken altijd prima op mijn gemak en gewaardeerd gevoeld. Ik heb mooie gesprekken en discussies gehad met verschillende van jullie en ik ben ook op allerlei vlakken geholpen door jullie, teveel om op te noemen. Dus FTE en WLR, bedankt.

Daarnaast wil ik alle 15 studenten bedanken voor hun inzet, kennis en kunde tijdens hun BSc-, MSc-thesis, of stage binnen mijn onderzoek. Zonder jullie had ik nooit al het werk gedaan kunnen krijgen.

Ook de melkveehouders bedankt voor jullie gastvrijheid en jullie interesse in mijn onderzoek. De belangrijkste waren echter toch wel "mijn eigen"melkveehouders. Pa en ma, bedankt voor de kansen, mogelijkheden en ondersteuning om te bereiken waar ik nu ben. En ook Jessica, mijn kleine grote zus, bedankt.

Ten slotte mijn allerbelangrijkste steun en toeverlaat. Mijn maatje, mijn beste vriendin. Mijn duw in de rug op de momenten dat het nodig is, en bovenal mijn vrouw. Eveline, bedankt. 


\section{About the author}

Dennis (Johannes Wilhelmus) Snoek was born on February 24, 1986 in Woerden, and grew up at the parental dairy farm. After obtaining his pre-university degree in 2004 at "Het Schoonhovens college" in Schoonhoven, he started with the BSc and then the MSc program "Biosystems Engineering" at Wageningen University. As part of his education he conducted a minor thesis at the Systems and Control Group. His major thesis took place at the Farm Technology Group and was about floor designs in dairy cow houses. For his internship he executed a market research for the milking equipment manufacturer company SAC. In January 2010, he started working for the Farm Technology Group at Wageningen University as a researcher and teacher, with the aim to start up a $\mathrm{PhD}$ in the field of ammonia emissions from dairy cow houses. In the first 1.5 year he mainly taught two courses, being the BSc course Engineering Design, and the MSc course Livestock Technology. Additionally, he obtained his University Teaching Qualification (UTQ). From August 2011 onwards his $\mathrm{PhD}$ officially started of which the results are presented in this thesis, and he continued teaching. Since September 2016 he works for the Agrifirm Innovation Centre, part of the Royal Agrifirm Group, as Researcher Ruminants.

Besides his study and professional career, he was rather active in several other disciplines. He organised activities and was chairman in the board for the Biosystems Engineering related study association "Heeren XVII". He was activity coordinator and treasurer for the WIAS Associated PhD Students (WAPS) Council. He actively played soccer, continued with mountain biking, and on the side he finished a triathlon. Currently he is working on the renovation of a typical Dutch, regional specific, historical dairy farm. 



\section{List of publications}

\section{Publications in peer-reviewed journals}

Snoek, D.J.W.; Ogink, N.W.M.; Stigter, J.D.; Agricola, S.; van de Weijer, T.M.; Groot Koerkamp, P.W.G. (2016). Dynamic behaviour of $\mathrm{pH}$ in fresh urine puddles of dairy cows. Transactions of the ASABE, 59(5).

Snoek, D.J.W.; Stigter, J.D.; Ogink, N.W.M.; Groot Koerkamp, P.W.G. (2015). Measurement method for urine puddle depth in dairy cow houses as input variable for ammonia emission modelling. CIGR Journal, Special issue: 18th World Congress of CIGR, Beijing, China, 30-36.

Snoek, D.J.W.; Stigter, J.D.; Ogink, N.W.M.; Groot Koerkamp, P.W.G. (2014). Sensitivity analysis of mechanistic models for estimating ammonia emission from dairy cow urine puddles. Biosystems Engineering, 121, 12-24.

\section{Accepted for publication}

Mendes, L.B.; Pieters, J.; Snoek, D.J.W.; Ogink, N.W.M.; Brusselman, E.; Demeyer, P. Reduction of ammonia emissions from free stall dairy cattle barns via improved management- or design- based strategies: A modeling approach. Science of the Total Environment.

\section{Submitted for publication}

Snoek, D.J.W.; Stigter, J.D.; Kupers, G.C.C.; Groot Koerkamp, P.W.G.; Ogink, N.W.M. Assessing fresh urine puddles in commercial dairy cow houses part 2: pH, urea concentration, and ammonia emission potential. 
Snoek, D.J.W.; Stigter, J.D.; Blaauw, S.K.; Groot Koerkamp, P.W.G.; Ogink, N.W.M. Assessing fresh urine puddles in commercial dairy cow houses part 1: surface area and depth.

Snoek, D.J.W.; Hofstee, J.W.; van Dueren den Hollander, A.W.; Vernooij, R.E.; Ogink, N.W.M.; Groot Koerkamp, P.W.G. Quantification of simulated cow urine puddle areas using a thermal IR camera.

\section{Conference proceedings}

Snoek, D.J.W.; Stigter, J.D.; Ogink, N.W.M.; Groot Koerkamp, P.W.G. (2014). Development of a measurement method for urine puddle area in dairy cow houses. In International Conference on Agricultural Engineering. Zürich, Switzerland.

Snoek, D.J.W.; Ogink, N.W.M.; Stigter, J.D.; Groot Koerkamp, P.W.G. (2012). Sensitivity Analysis of a Mechanistic Model for the Ammonia Emission of Dairy Cow Houses. In IX International Livestock Environment Symposium (ILES IX). Valencia, Spain.

Snoek, J.W.; Haesen, G.P.M.J.; Groot Koerkamp, P.W.G.; Monteny, G.J. (2010). Effect of floor design in a dairy cow house on ammonia emission-design, test and preliminary results with an experimental set-up for run off experiments. In International Conference on Agricultural Engineering. Clermont-Ferrand, France.

\section{Other publications}

Snoek, D.J.W. (2013). Gevoeligheidsanalyse van ammoniak emissiemodel voor melkveestallen - Onderscheiden van relevante invoervariabelen in het rekenmodel. Interne Notitie. Pag. 40. Wageningen.

Aarnink, A.J.A.; Harn, J. van; Hattum, T.G. van; Zhou, Y.; Snoek, J.W.; Vermeij, I.; Mosquera Losada, J. (2008). Reductie stofemissie bij vleeskuikens door aanbrengen oliefilm. Animal Sciences Group Rapport 154. Pag. 42. 


\section{Training and Supervision Plan}

\begin{tabular}{|c|c|c|}
\hline Topic and location & year & ECTS ${ }^{\dagger \ddagger}$ \\
\hline \multicolumn{3}{|l|}{-Basic package- } \\
\hline WIAS introduction course & 2011 & 1.5 \\
\hline Course on philosophy of science and/or ethics & 2013 & 1.5 \\
\hline \multicolumn{3}{|l|}{-International conferences- } \\
\hline International Conference on Agricultural Engineering, Clermont-Ferrand, France & 2010 & 0.9 \\
\hline International Conference of Agricultural Engineering, Valencia, Spain & 2012 & 0.9 \\
\hline \multicolumn{3}{|l|}{ Engineering, Bejing, China } \\
\hline \multicolumn{3}{|l|}{-Seminars and workshops- } \\
\hline $\begin{array}{l}\text { International workshop on the use of flux chambers for emission measurements } \\
\text { in naturally ventilated livestock housing, Wageningen }\end{array}$ & 2009 & 0.6 \\
\hline Workhop NH3 in livestock systems (ASG, INRA, CemaGref), Rennes, France & 2011 & 0.6 \\
\hline Wias Science Day 2012, 2013, 2014, 2015, Wageningen & - & 0.3 \\
\hline $\begin{array}{l}\text { Self organised "farmers-day" to inform dairy farmers, Wageningen } \\
\text {-Presentations - Oral- }\end{array}$ & 2015 & - \\
\hline $\begin{array}{l}\text { Effect of floor design in a dairy cow house on ammonia emission, } \\
\text { Clermont-Ferrand, France }\end{array}$ & 2010 & 1.0 \\
\hline TAC-RAV model presentation, start and approach, Utrecht & 2010 & 1.0 \\
\hline Determine ammonia emission from a dairy cow house, Rennes, France & 2011 & - \\
\hline TAC-RAV model presentation, model version 1.0, Utrecht & 2011 & - \\
\hline Parameter sensitivity of NH3 emission model, Valencia, Spain & 2010 & 1.0 \\
\hline $\begin{array}{l}\text { Sensitivity analysis of a mechanistic model for estimating ammonia emission } \\
\text { from dairy cow houses, WIAS Science Day, Wageningen }\end{array}$ & 2014 & 1.0 \\
\hline Measurement method for urine puddle depth in dairy cow houses, Beijing, China & 2014 & 1.0 \\
\hline $\begin{array}{l}\text { Development of an assessment strategy to determine the ammonia emission from } \\
\text { dairy cow houses, invited speaker in the project Environmental Dairy Design } \\
2020 \text {, Wageningen }\end{array}$ & 2015 & - \\
\hline Ammoniakemissie infodag, speaker at my own "farmers-day", Wageningen & 2015 & - \\
\hline Ammoniakemissie stalmetingen, update TAC-RAV, Utrecht & 2015 & - \\
\hline
\end{tabular}


Topic and location

year ECTS ${ }^{\dagger \ddagger}$

-Presentations - Poster-

Development of a measurement method for urine puddle area in dairy cow

$2014-$

houses, Zurich, Switzerland

Sensitivity analysis for NH3 emission from dairy cow urine puddles, Biometris 2014 -

$\mathrm{PhD}$ day, Wageningen

Development of a measurement method for urine puddle area in dairy cow 2015 -

houses, WIAS Science Day, Wageningen

-Research skills training and In-Depth Studies-

Preparing own PhD research proposal

20116.0

Fundamentals of Ventilation, Indoor Air Quality, Air Motion and Emissions; 201210

NOVA university network; Aarhus University; Viborg, Danmark

-Professional Skills Support Courses-

Competence Assessment

$2012 \quad 0.3$

Scientific writing

20121.8

Techniques for Writing and Presenting a Scientific Paper

$2012 \quad 1.2$

University Teaching Qualification (Basis Kwalificatie Onderwijs BKO)

$2012 \quad 4.5$

- Teaching and Supervising Thesis students

20110.6

- Conversation skills in one-to-one guidance

$2011 \quad 0.6$

- General course on Teaching and Learning

20113.5

- Designing exams

$2011 \quad 0.6$

Career orientation

$2015 \quad 1.5$

-Lecturing, Supervising practicals and excursions, and preparing course material-

Teacher and coordinator of FTE-24806. Period 1 2010-2015, appr. $180 \mathrm{~h} /$ year -

Teacher and coordinator of FTE-30306. Period 4 2010-2012, appr. 80 h/year

Practicals and Excursion in FTE-24806, appr. $60 \mathrm{~h} /$ year

Practicals and Excursion in FTE-30306, appr. $36 \mathrm{~h} /$ year

Design of FTE-24806: lectures, cases, exercises, and exams. Appr. $240 \mathrm{~h}$

Design of FTE-30306: exams. Appr. $16 \mathrm{~h}$

-Supervising theses-

Supervising MSc Major Thesis (2 students)

Supervising BSc Thesis (12 students) $\quad-\quad 2.0$

$-\quad 4.0$

Supervising internship (1 student; agricultural university of applied sciences) - -

-Management Skills Training-

Organised a "Farmers day" to inform dairy farmers

WAPS council member: accie and treasurer. 2012 and 2013.

$2015-$

(not held)

-Education and Training Total-

$53.8^{\dagger \ddagger}$

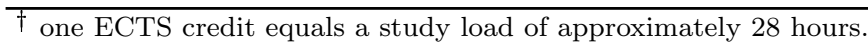

$\ddagger$ not all activities were counted for in ECTS. 
Notes 

Cover and layout design: Dennis J.W. Snoek Printed by: GVO drukkers \& vormgevers B.V.

Copyright (C) Dennis Snoek, 2016 



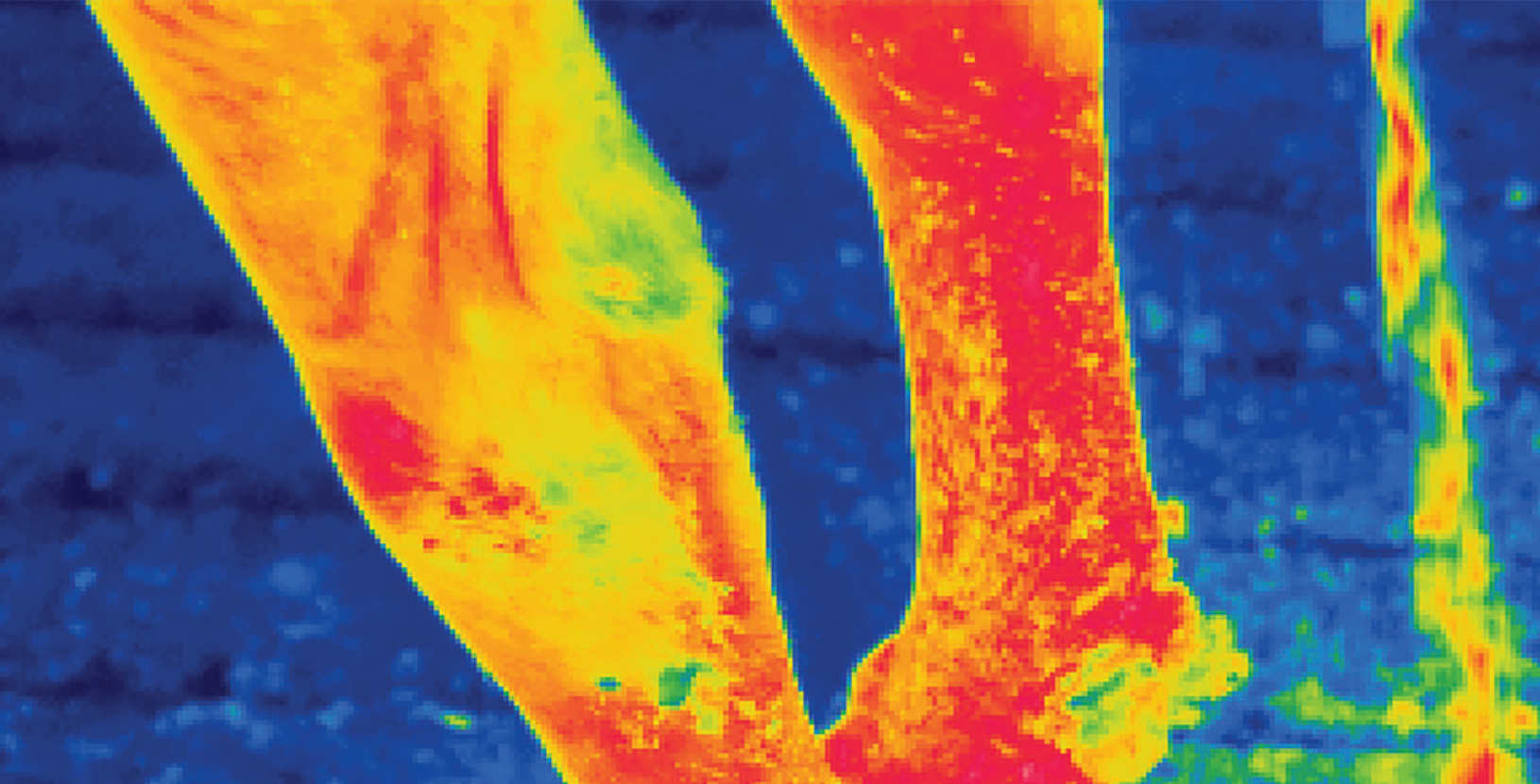

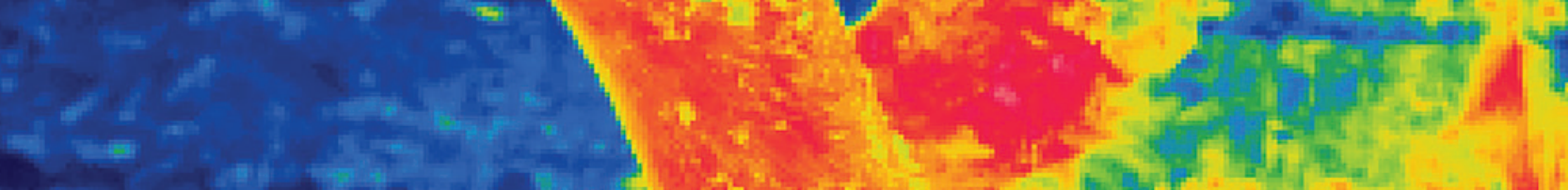

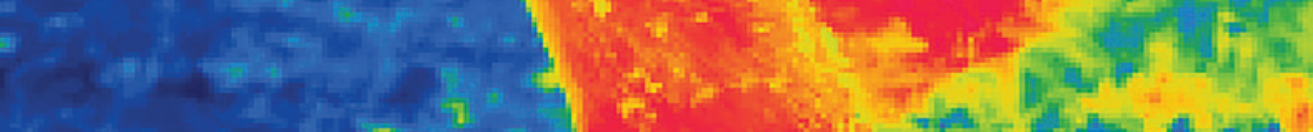

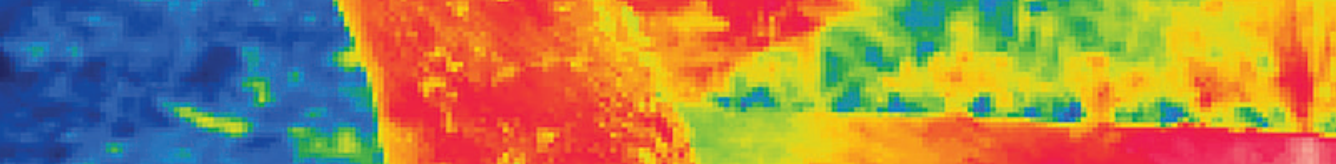

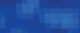

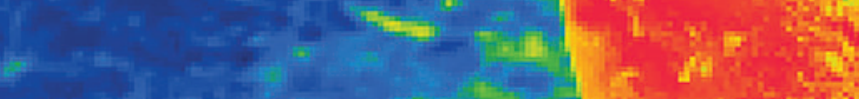

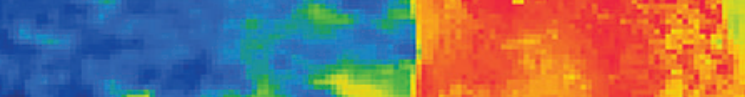

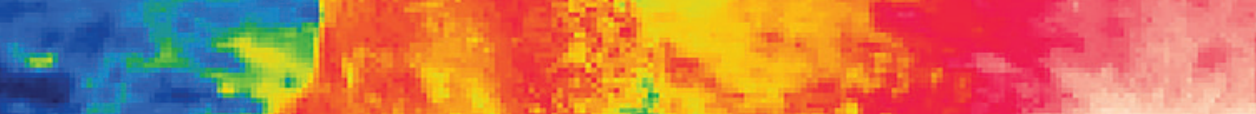

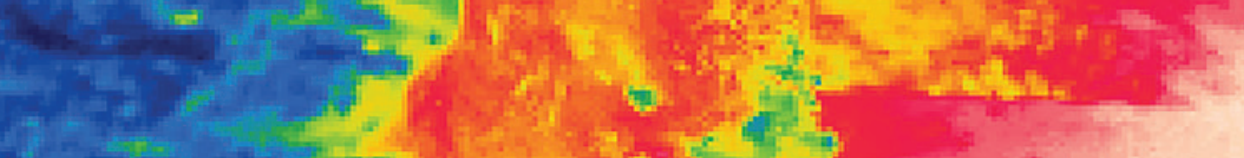
(s)ate? 204.4

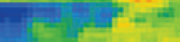

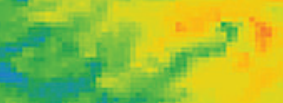

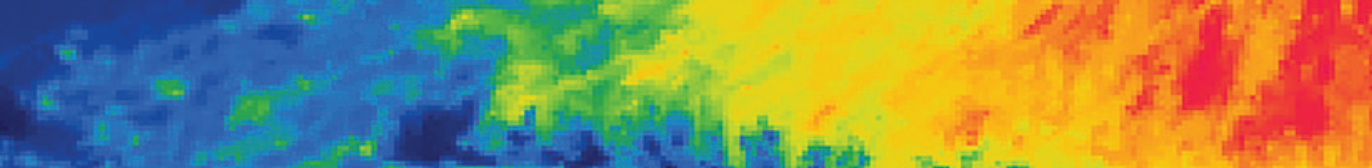
(3)

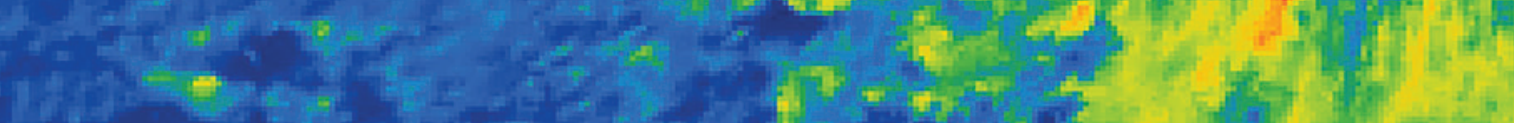

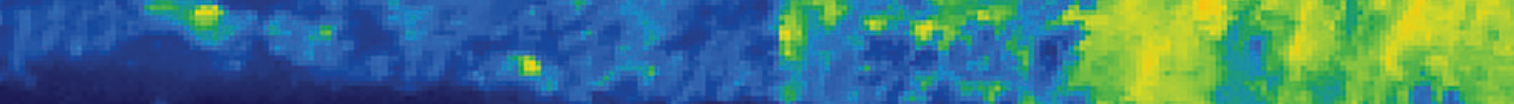

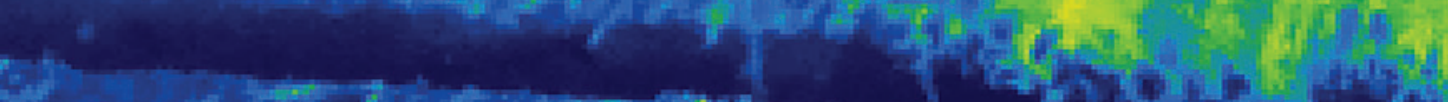

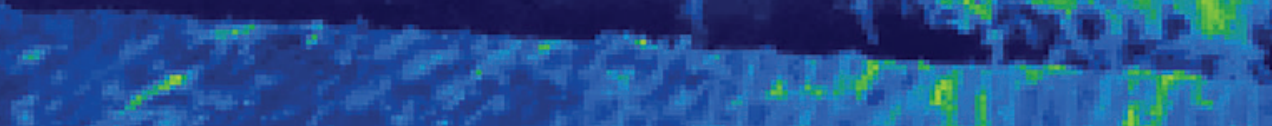
14:

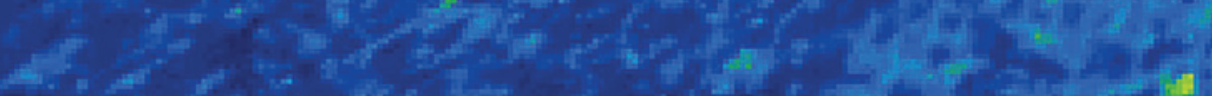

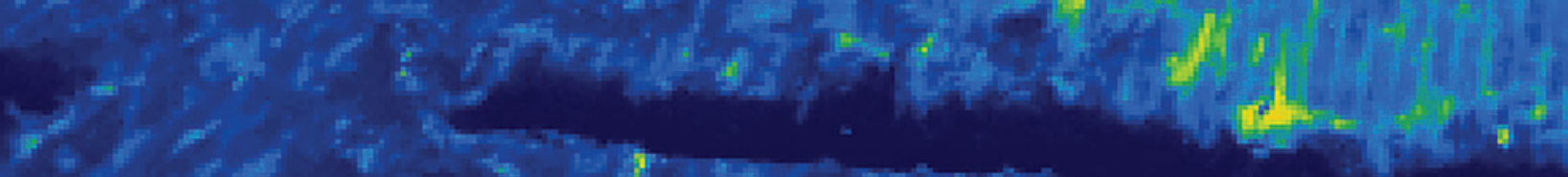

